\title{
New Band-gap Measurement Technique for a Half-Metallic Ferromagnet
}

Tariq Fahad H. Alhuwaymel

$\mathrm{PhD}$

University of York

Electronics

July 2015 


\section{Abstract}

Spintronics is a new and emerging field in nanotechnology, which has been evolving rapidly. It aims to exploit the spin degree of freedom in order to realise advanced electronic devices. With the recent improvement in the storage media devices following to the discovery of giant magnetoresistance effect, it is envisioned that the electronic devices have several advantages over the conventional electronics in respect to the storage capacity, speed and power consumption. One avenue towards next generation spintronic devices is to develop half-metallic ferromagnets (HMFs) with $100 \%$ spin polarisation $(P)$ and Curie temperature $\left(T_{\mathrm{C}}\right)$ above room temperature $(\mathrm{RT})$. HMFs have unique properties, in which the majority spins have a metallic band structure, whereas the minority spins have a semiconducting band with the Fermi level $\left(E_{\mathrm{F}}\right)$ lying within an energy gap. $P$ of HMFs has been predominantly estimated using Julliere's formula in a magnetic tunnel junction (MTJ) or measured by the Andreev reflection (AR) at low temperature. Both methods are very sensitive to the surface/interface spin polarisation. Alternative optical methods such as photoemission have also been employed. However, these methods require a complicated and expensive set-up. Therefore, it is of paramount importance to directly and easily measure the band-gap of HMFs.

The main aim of this study is to develop a new technique to directly measure the band-gap $\left(E_{\mathrm{g}}\right)$ of HMFs at RT. For that, a simple experimental set-up has been designed utilising circularly-polarised infrared (IR) light to excite minority spins and measure the corresponding absorption. The validity of our technique was demonstrated by measuring $E_{\mathrm{g}}$ of polycrystalline and epitaxial full-Heusler-alloy films and single-crystal half-Heusler alloy bulk. $E_{\mathrm{g}}$ measurements have revealed relevant figures to the calculated and expected reported values.

This technique allows measuring $E_{\mathrm{g}}$ of HMFs at RT for the first time. It can therefore be used to provide simple optimisation of growth conditions. 


\section{Contents}

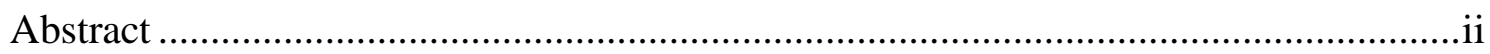

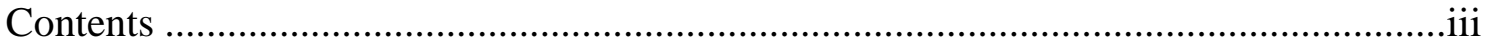

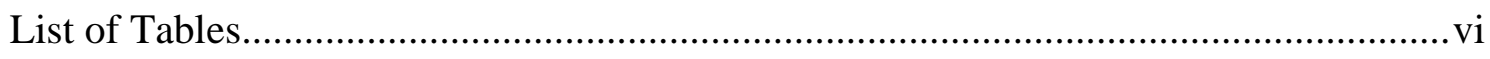

List of Figures ...........................................................................................vii

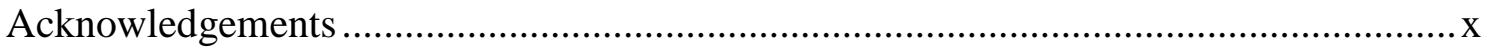

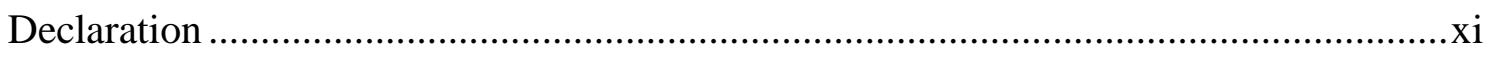

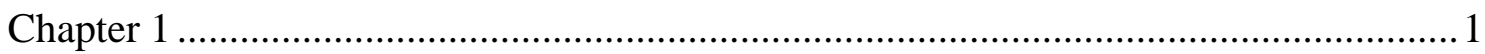

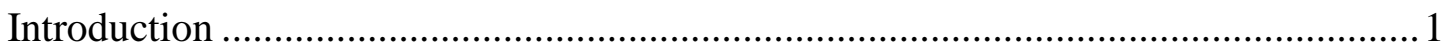

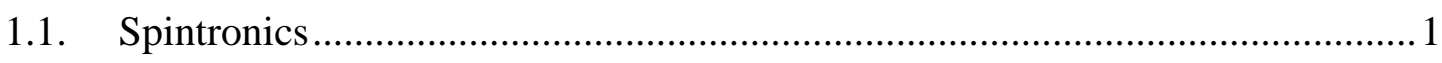

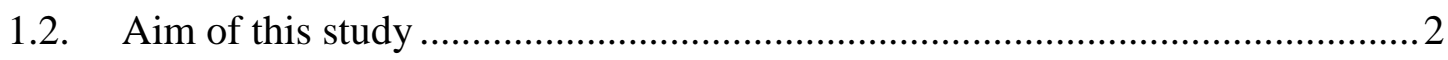

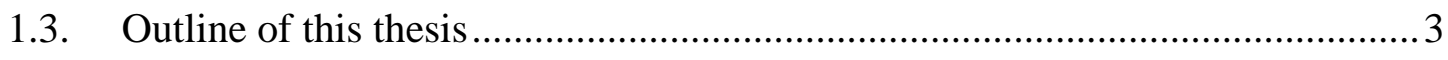

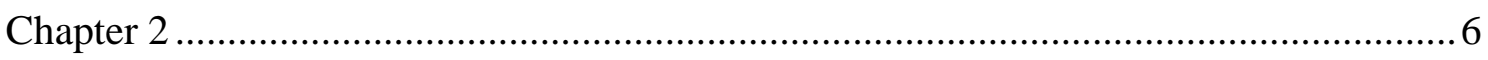

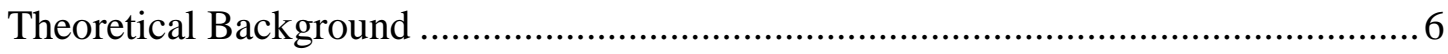

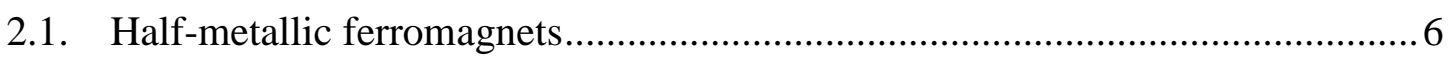

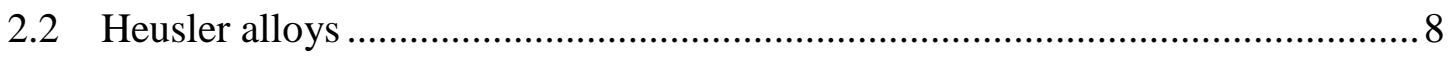

2.2.1 Origin of the band-gap in the full-Heusler alloys ........................................ 10

2.2.2 Generalised Slater-Pauling behaviour ………………………………......... 11

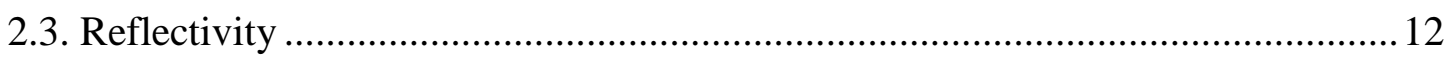

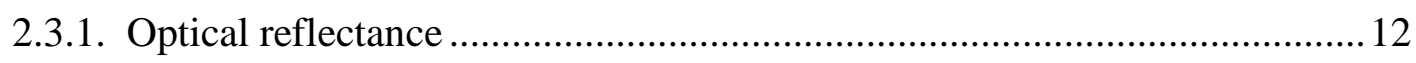

2.3.2. Determination of $\epsilon(\omega)$ from the measured reflectivity ................................ 13

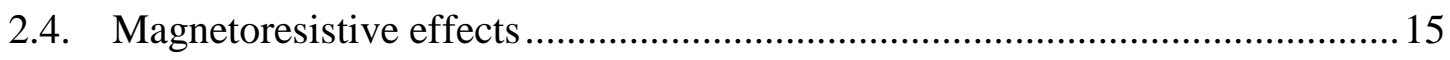

2.4.1 Tunnelling magnetoresistance (TMR) ....................................................... 15

2.4.2 Giant magnetoresistance (GMR) …………………………………...... 17

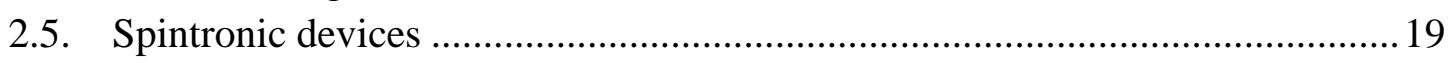

2.5.1. Magnetic random access memory (MRAM) ………….............................. 19

2.5.2. Spin-polarised field effect transistor (spin-FET) ........................................ 21

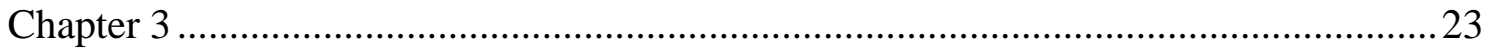

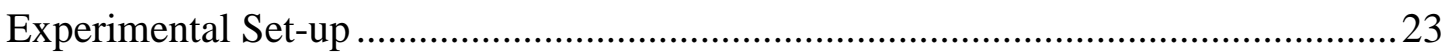

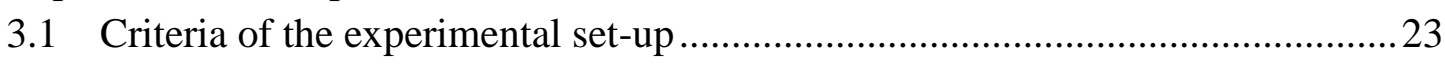

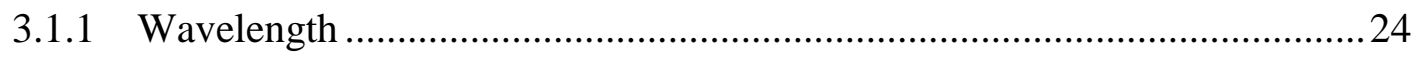

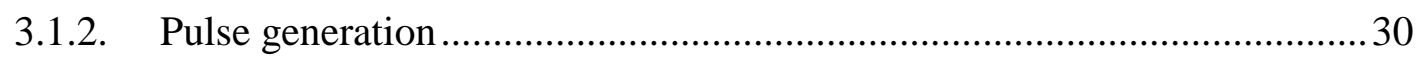

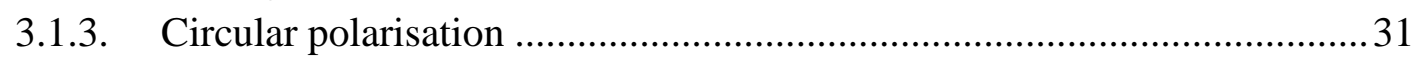

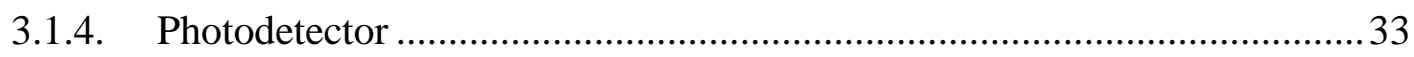

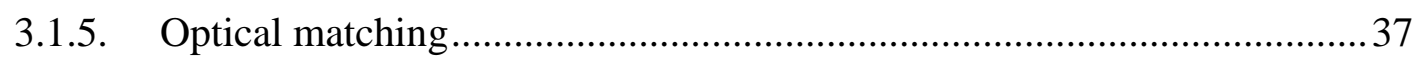

3.1.6. Lock-in technique ............................................................................ 38

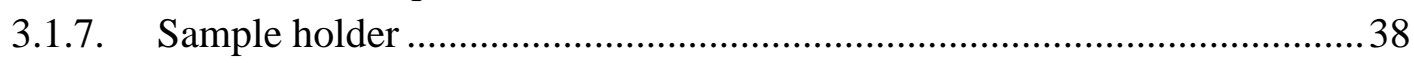

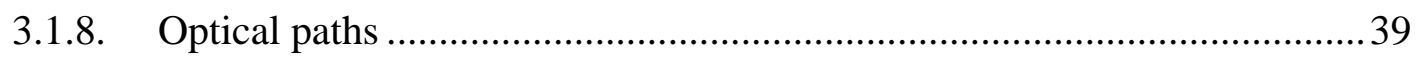

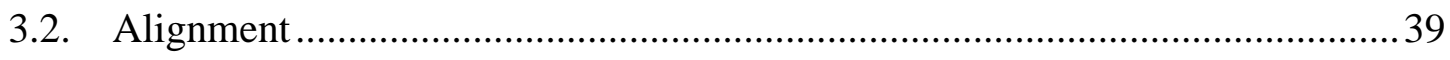




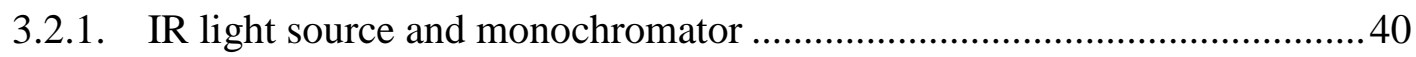

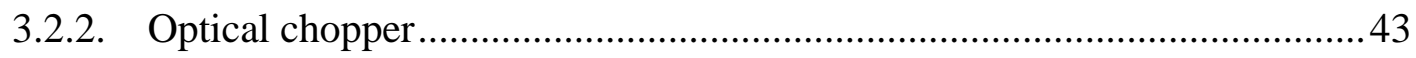

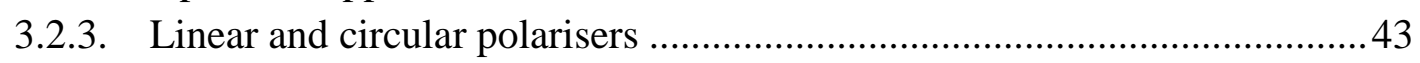

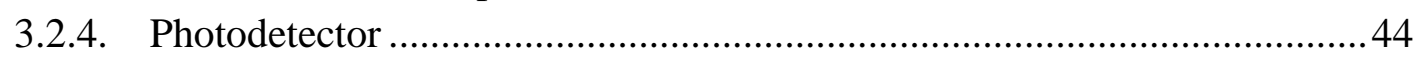

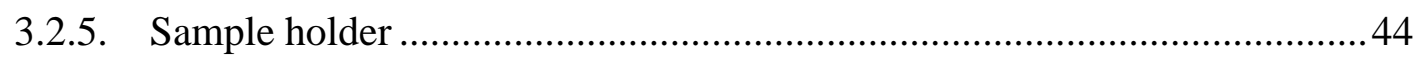

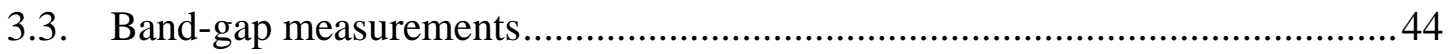

3.4. Measurements improvement .................................................................. 45

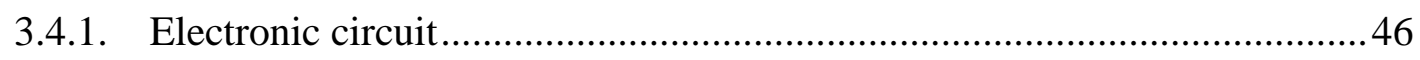

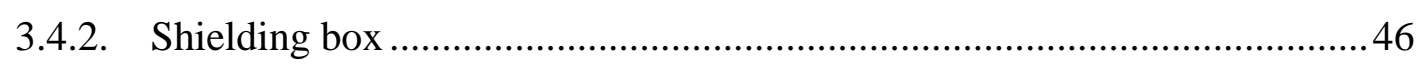

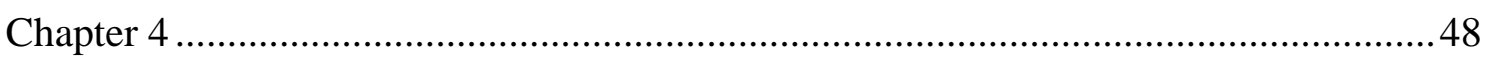

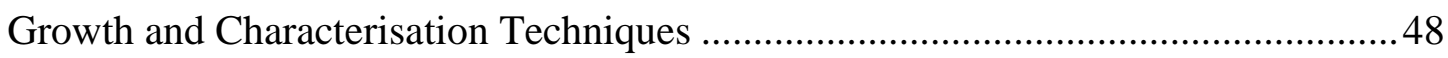

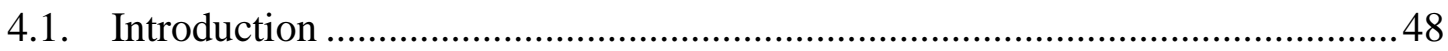

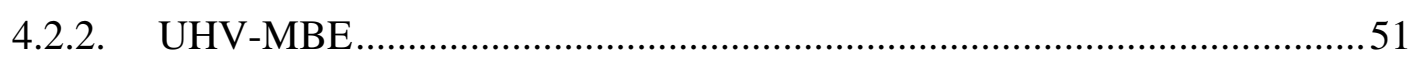

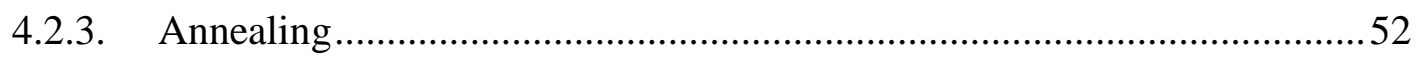

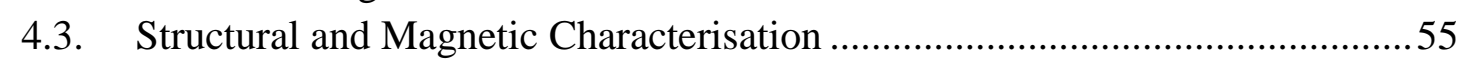

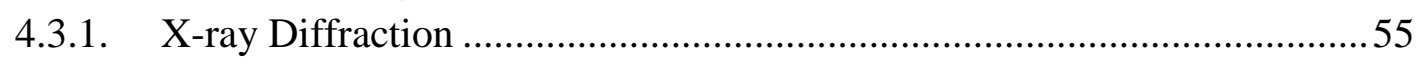

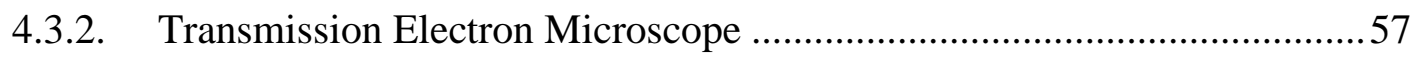

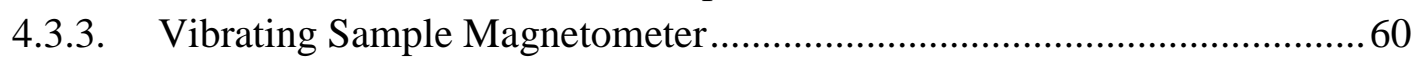

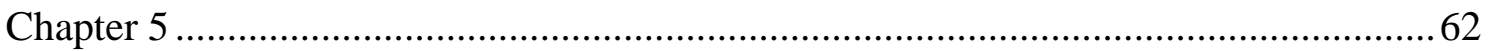

Heusler Band-Gap Measurements on Polycrystalline $\mathrm{Co}_{2} \mathrm{FeSi}$ Films ........................62

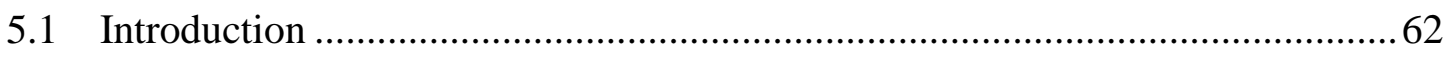

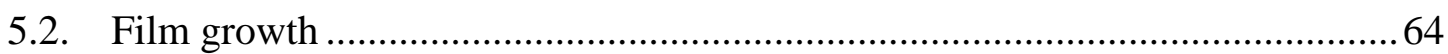

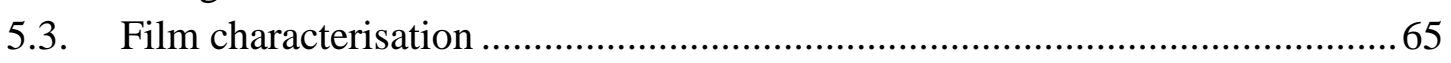

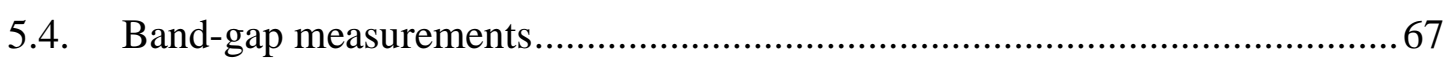

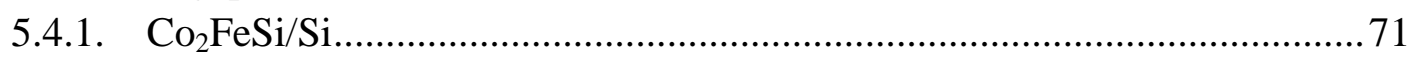

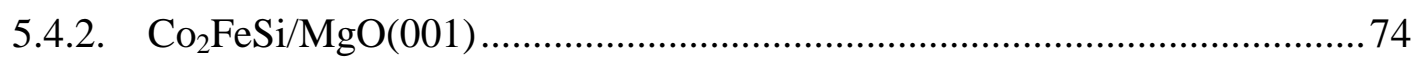

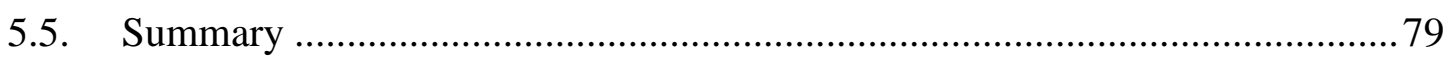

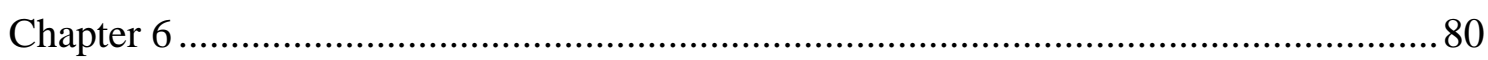

Heusler Band-Gap Measurements on Epitaxial $\mathrm{Co}_{2} \mathrm{FeAl}_{0.5} \mathrm{Si}_{0.5} / \mathrm{MgO}(001)$................8 80

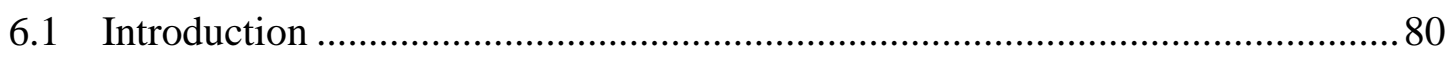

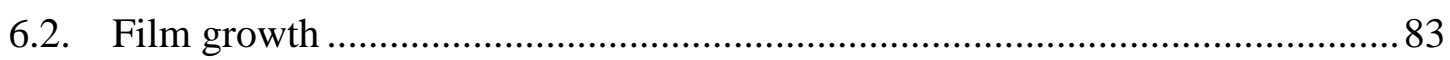

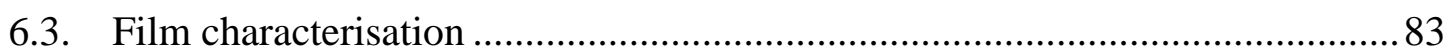

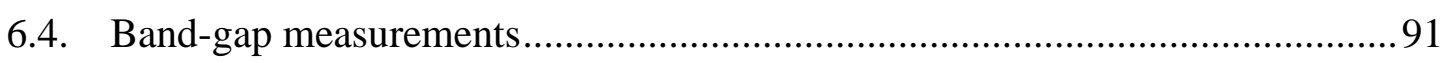

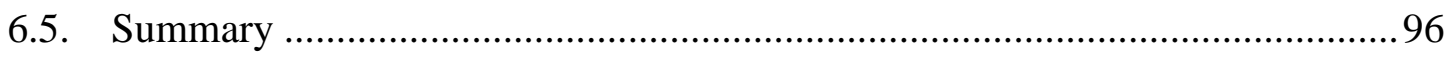

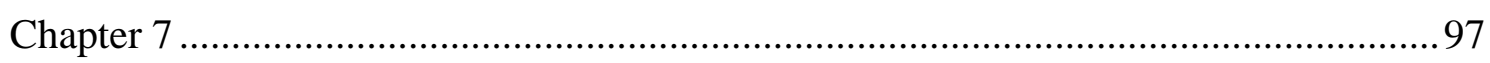

Heusler Band-Gap Measurements on a NiMnSb Heusler Alloy................................ 97

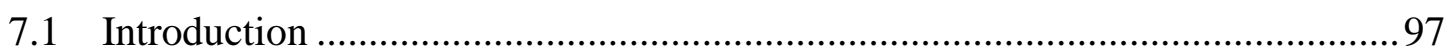

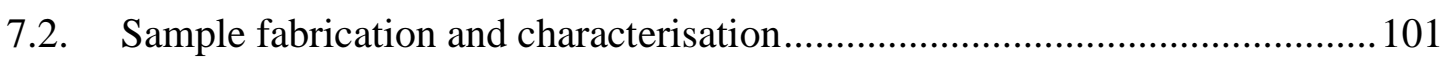

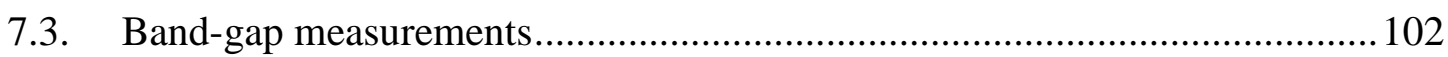

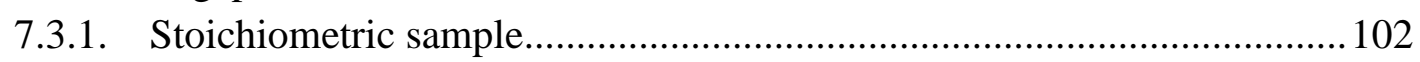

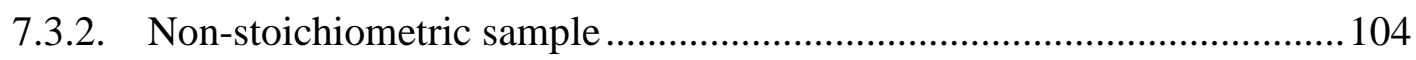

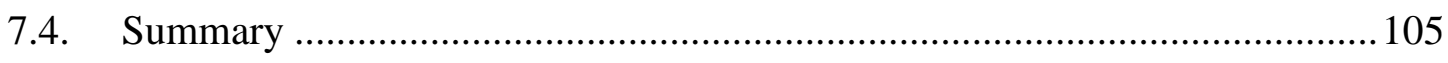

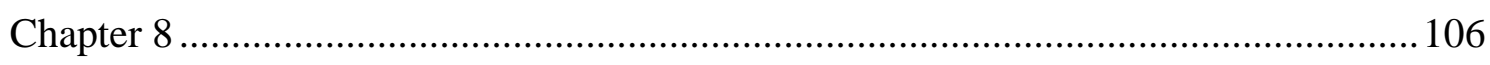

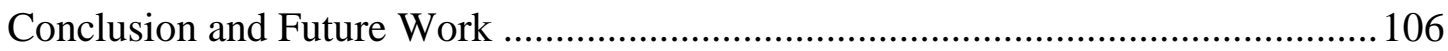




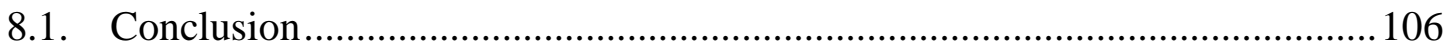

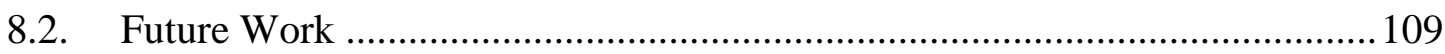

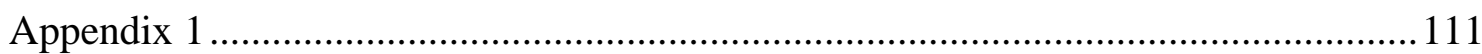

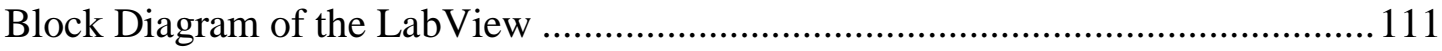

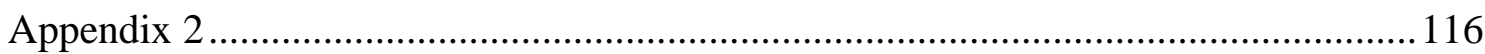

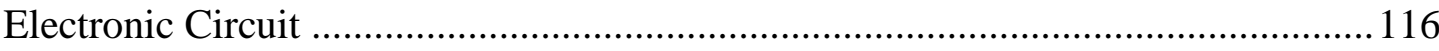

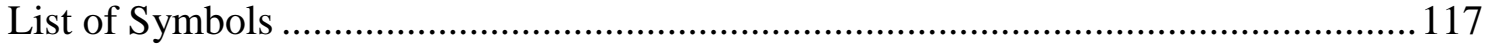

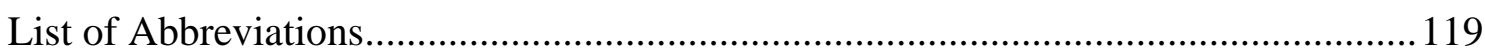

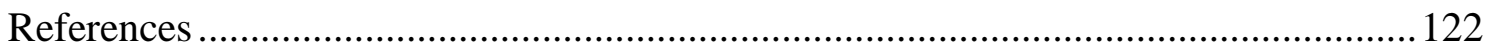




\section{List of Tables}

List of major magnetic properties of studied Heusler alloys. ............................................. 24

Comparison of different types of detectors. ................................................................... 33

List of experimental and theoretical magnetic properties of CFS ........................... 59

List of calculated magnetic properties of CFAS. ………….............................................. 76

List of magnetic properties of CFAS obtained experimentally. ....................................... 76

List of experimental and theoretical magnetic properties of NMS. ............................... 94 


\section{List of Figures}

Density of states of $\mathrm{Co}_{2} \mathrm{FeSi}$.

Crystalline structures of two types of Heusler alloys.

Curie temperature distribution of full-Heusler with respect to the element X.

Lattice constant distribution of full-Heusler with respect to the element X.

Schematic illustration of the origin of band-gap in full-Heusler alloys.

Calculated total spin moments for full-Heusler alloys.

Schematic diagrams of an MTJ and the corresponding TMR effect.

Schematic diagram of CIP- and CPP-GMR measurements.

Schematic diagram of MRAM.

Schematic diagram of spin-FET.

Wavelength dependence of emissivity for the IR light source.

Configuration of the IR lamp, the reflector mirror and the monochromator. 26

Schematic diagram of the monochromator. 26

Efficiency distribution for the two diffraction gratings.

Signals of the optical chopper. 28

Typical maximum transmission for linearly polarised light. 29

Maximum transmission of the KRS-5 linear polariser.

Schematic diagram of the $\lambda / 4$-wave plate. 30

Wavelength dependence of the transmission efficiency for $\lambda / 4$-wave plate. 30

Transmission characteristics of the IR photodetector. 33

Response of the photodetector at different view angles. 33

Pyroelectric phototdetector with various feedback resistor values. 34

Schematic diagram of the experimental set-up. 37

Reference intensity distribution of the IR light source. 38

Measured intensity distribution of the IR light source at $20 \mathrm{~nm}$ steps. 38 
Reference intensity distribution of the IR light source.

The measured IR light intensity with and without reflector.

Photodetector in the aluminium box.

The base of the shielding box.

Schematic diagram of the HiTUS system.

Schematic diagram of the MBE system.

Photograph of the furnace.

Configuration of XRD scans.

Schematic diagrams of the two basic operation modes of TEM. 54

Block diagram of the VSM.

XRD intensities for the CFS films. 62

TEM images and electron diffraction patterns for the CFS films.

Hysteresis loops for the as-deposited CFS film. 63

Measured intensities for as-deposited CFS films between 3 and $9.5 \mu \mathrm{m}$. 66

Measured intensities for as-deposited CFS films 9.5 and $16 \mu \mathrm{m}$. 67

Measured intensities for the as-deposited CFS/Si films. 68

Intensities for the as-deposited CFS/Si films and their differences. 69

Intensity differences for the CFS/Si films. 70

Measured intensities for the as-deposited CFS/MgO(001) films. 71

Intensities for as-deposited $\mathrm{CFS} / \mathrm{MgO}(001)$ films and their differences. 72 Intensity differences for the as-deposited and after post-annealing $\mathrm{CFS} / \mathrm{MgO}(001)$ films. 73

Annealing time dependence of the intensity difference. 74

XRD intensities of the 6-h annealed $\mathrm{CFAS} / \mathrm{MgO}(001)$ films using different scan configurations. 
XRD analysis of the 6-h annealed CFAS/MgO(001) films using different scan configurations.

RHEED pattern and cross-sectional TEM image with the corresponding SAED pattern of the as-deposited CFAS films. 82

Hysteresis loops the as-deposited and 6-h annealed CFAS/MgO(001) films 85

Annealing time dependence of $M \mathrm{~s}$ of the CFAS/MgO(001) films. 85

Measured intensities for the as-deposited CFAS/MgO(001) films. 86

Intensities for the as-deposited CFAS/MgO(001) films and their differences. 87

Differences in intensities for CFAS/MgO(001) films after post-annealing. 89

Weak-beam dark-field image of $\mathrm{CFS} / \mathrm{MgO}(001)$ interface. 90

Annealing time dependence of band-gap energies of $\mathrm{CFAS} / \mathrm{MgO}(001)$ films. 90

Measured intensities for the stoichiometric bilk NiMnSb. 98

Photograph of the bulk NiMnSb sample. 98

Measured intensities for the non-stoichiometric bulk NiMnSb. 99

Interface of the LabView programme for the band-gap measurements. 108

Block diagram of the first step of the band-gap measurement to create a file to save the measured data. 109

Block diagram of the initialisation of the monochromator. 109

Block diagram of the set-up of the lock-in amplifier. 110

Block diagram of the selection of the parameter values for the lock-in amplifier.

Block diagram of the execution of the measurement with the control of all the devices connected.

Final electronic circuit, which has been developed in this study. 


\section{Acknowledgements}

At the end of my $\mathrm{PhD}$ research journey, I would like to express my sincere gratitude to my first supervisor Prof. Atsufumi Hirohata for his invaluable support during my $\mathrm{PhD}$ research study and writing up this thesis, for his patience, encouragement, enthusiasm, and immense knowledge. I could not have imagined having a better supervisor for my $\mathrm{PhD}$. His relentless pursuit of perfection has made me a better researcher. Besides my first supervisor, I would like to give my gratitude to my second supervisor, Prof. Mohamed El-Gomati for his kind support, suggestions, insightful comments and encouragement.

I would like also to extend my sincere thankfulness to Prof. Kevin O'Grady for allowing me using his labs. My thanks go to Prof. Sarah Thompson, the Head of Physics Department of the University of York, for helping me in the first year of my $\mathrm{PhD}$ to find a proper photodetector. I also wish to thank the staff in the workshops in the Electronics and Physics Departments especially Mr. Dave Coulthard and Mr. Andy White. I also acknowledge the help from other members of the group particularly to Drs. Luke Fleet, James Sagar and Robert Carpenter.

Special thanks go to Dr. Turki S. M. Al Saud, President of King Abdulaziz City for Science and Technology (KACST), for his kind support to allow me to obtain my scholarship. A great thank to my lifetime friend, Eng. Abdullah Alyousef for his help, encouragement and the fun he provided for me. Similarly thanks to my best friends; Dr. Sherif Bakush, Dr. Hamid Al-Garni and Dr. Ranjdar Abdullah for their fruitful discussions, encouragement, help and hope.

Finally, I would like to thank all my family, my sons and friends, and my warm gratitude from my heart to my wife, Mrs. Fatima Almodhi for her love, patience, reassurance and support. 


\section{Declaration}

I declare that the work presented in this thesis is based purely on my own research, unless otherwise stated, and has not been submitted for a degree in either this or any other university. Some of the research presented in this thesis has resulted in the following publications:

- T. F. Alhuwaymel, R. M. Abdullah, O. Whear, T. Huminiuc, R. Carpenter, M. ElGomati and A. Hirohata, "New Bandgap Measurement Technique for a HalfMetallic Ferromagnet”, IEEE transactions on Magnetics 50, 2600504 (2014).

- T. F. Alhuwaymel, R. Carpenter, C. N. T. Yu, B. Kuerbanjiang, R. M. Abdullah, V. K. Lazarov, M. El-Gomati and A. Hirohata, "Direct band-gap measurement on epitaxial $\mathrm{Co}_{2} \mathrm{FeAl}_{0.5} \mathrm{Si}_{0.5}$ Heusler-alloy films", Journal Applied Physics 117, 17D131 (2015). 


\section{Chapter 1}

\section{Introduction}

\subsection{Spintronics}

Spin-based electronics, spin electronics or magnetoelectronics are all different names for "spintronics" [1][2]. Spintronics is a new and emerging field that aims to realise high-performance electronic devices by utilising electron spins which used to be ignored in the conventional electronics. Recently, spintronics has attracted increasing numbers of researchers around the world, associated with many conferences and publications. Therefore, it can be considered as one of the most promising fields for the development of new materials and devices. This is evidenced by the significant improvement in the storage capacity of hard disk drives (HDD), which is based on the breakthrough discovery of giant magnetoresistance (GMR) [3][4] and the consequent improvement of tunnelling magnetoresistance (TMR) (see Sections 2.4.1 and 2.4.2, respectively).

In conventional electronic devices, the charge of electrons is used and the spin directions of electrons are random. Typically a half of electrons in the electronic devices have a spin-up direction and the other half has a spin-down direction, therefore the net spin polarisation is zero in a non-magnetic material. In a ferromagnetic material to be used in spintronics, on the other hand, the number of the majority "up-spin" and the 
minority "down-spin" electrons are not equal at $E_{\mathrm{F}}$ and therefore net spin polarisation can be created by the imbalance.

The efficiency of spintronic devices depends on the spin polarisation of ferromagnetic materials used. $P$ is defined as the difference between the number of upspin and down-spin electrons at $E_{\mathrm{F}}$, and can be expressed by the following simple formula [5]:

$$
P=\frac{N_{\uparrow}\left(E_{\mathrm{F}}\right)-N_{\downarrow}\left(E_{\mathrm{F}}\right)}{N_{\uparrow}\left(E_{\mathrm{F}}\right)+N_{\downarrow}\left(E_{\mathrm{F}}\right)},
$$

where $N_{\uparrow}$ and $N_{\downarrow}$ are the densities of the majority and minority electrons at $E_{\mathrm{F}}$, respectively.

$100 \%$ spin-polarised materials are one of the essential requirements in order to improve the efficiency of spintronics devices. Due to the presence of a band-gap only in one spin band, HMFs are expected to exhibit $100 \%$ spin polarisation [5]. Hence, HMFs are the best candidate for spintronic devices [4].

Until recently the spin polarisations of HMFs have been estimated using Julliere's formula in a MTJ to be discussed in Section 2.3.1. [6] or measured by the Andreev reflection [7], both of which are very sensitive to surface/interface spin polarisation. Several other methods, such as a tunnel contact with a superconductor [8], spin-resolved photoemission [9] and optical pump-probe [10] techniques have been reported. However, these techniques require either low temperature or complicated setups. It is therefore critical to develop a new simple technique to measure the semiconducting band-gaps in HMF, i.e., $100 \%$ spin polarisation with a band-gap to be measured.

\subsection{Aim of this study}

The main aim of this study is to develop a new technique to directly measure $E_{\mathrm{g}}$ of 
HMFs at RT. In order to achieve this aim, an experimental set-up has been designed in such a way that circularly-polarised IR light excites the valence-band electrons to the conduction band across $E_{\mathrm{g}}$. In this study, due to both viability and simplicity, a reflection mode has been selected to measure the changes in the reflected signals from the sample surface and these signals are measured to evaluate the presence of a bandgap. This developed set-up can also be used to measure the band-gap electrically by detecting the change in the four-point-probe signals induced by the optical absorption at $E_{\mathrm{g}}$

In order to prove the feasibility of this new technique, three different samples have been selected for the $E_{\mathrm{g}}$ measurements. These are polycrystalline and epitaxial full-Heusler-alloy films and single-crystal half-Heusler alloy bulk. They have been selected due to their well-studied magnetic properties and potentials to be used in future spintronic devices.

The new technique developed in this study has several advantages; (i) flexible design, in which the $E_{\mathrm{g}}$ measurements can be conducted at various incidence angles by modifying the set-up, (ii) high sensitivity in the order of few $\mu \mathrm{V}$ and (iii) applicability for both thin films and bulk materials. This technique can therefore be used to analyse HMFs very easily and can provide feedback to their growth and preparation process for their optimisation.

\subsection{Outline of this thesis}

The thesis consists of 8 chapters and the outline is as follows:

Chapter 2 gives theoretical background on HMFs with more focus on Heusler alloys as one of four types that have theoretically been predicted to be HMFs. This chapter also covers the origin of a band-gap in full-Heusler alloys, generalised SlaterPauling behavior and magnetoresistance effects, including TMR and GMR. At the end 
of this chapter, two examples of spintronic devices, i.e., a magnetic random access memory (MRAM) and a spin-polarised field effect transistor (spin-FET), are explained.

Chapter 3 is devoted for the experimental set-up of the band-gap measurement technique developed in this study. This chapter is divided into four subsections. The first subsection gives the detailed criteria for the measurement set-up to satisfy; (i) wavelength, (ii) pulse generation, (iii) circular polarisation, (iv) a photodetector, (v) optical matching, (vi) a lock-in technique, (vii) a sample holder and (viii) optical paths. The second one explains the alignment procedures of the optical components; (i) an infrared (IR) light source and a monochromator, (ii) an optical chopper, (iii) linear and circular polarisers and (iv) a photodetector and a sample holder. The third explains how to conduct the band-gap measurements. The last subsection shows how to improve the measurements by modifying electronic circuits for the photodetector and by shielding the existing experimental set-up electromagnetically.

Chapter 4 describes the growth and characterisation techniques used in this thesis. The first half introduces two growth techniques used; (i) high target utilisation sputtering (HiTUS) and (ii) ultra-high vacuum (UHV) molecular beam epitaxy (MBE) with a post-annealing process. The latter half explains structural and magnetic characterisation using X-ray diffraction (XRD), transmission electron microscopy (TEM) and vibrating sample magnetometry (VSM).

Chapters 5 to 7 explain the results of the band-gap measurements on three different samples; polycrystalline $\mathrm{Co}_{2} \mathrm{FeSi}$ (CFS) and epitaxial $\mathrm{Co}_{2} \mathrm{Fe} \mathrm{Al}_{0.5} \mathrm{Si}_{0.5}$ (CFAS) full-Heusler-alloy films, and single-crystal NiMnSb (NMS) half-Heusler alloy bulk. The growth processes of the polycrystalline CFS films using HiTUS, the growth of the epitaxial CFAS films using UHV-MBE and the preparation of the NMS single crystal using arc-melting are also described in each chapter. The results of the structural and magnetic characterisation of the samples are then discussed. Especially, the evolution of 
these results with annealing is detailed. Finally, the measured band-gaps of the samples are discussed in relation to these results.

Chapter 8 concludes this study and provides the key achievements of the bandgap measurements on the three samples. It also shows future works to be carried out. 


\section{Chapter 2}

\section{Theoretical Background}

\subsection{Half-metallic ferromagnets}

HMFs are new class of materials that have unique property of having two distinctive band structures: the majority spin band (usually represented as up spins) have a metallic structure, while the minority spin band (down spins) have a semiconducting structure with a band gap at $E_{\mathrm{F}}[4-6][11]$ as shown in Fig. 2.1. Thus, they exhibit $100 \%$ spin polarisation at $E_{\mathrm{F}}$. Therefore, HMFs can be considered as an ideal spin injector into nonmagnetic materials and accordingly increase the efficiency of spintronics devices.

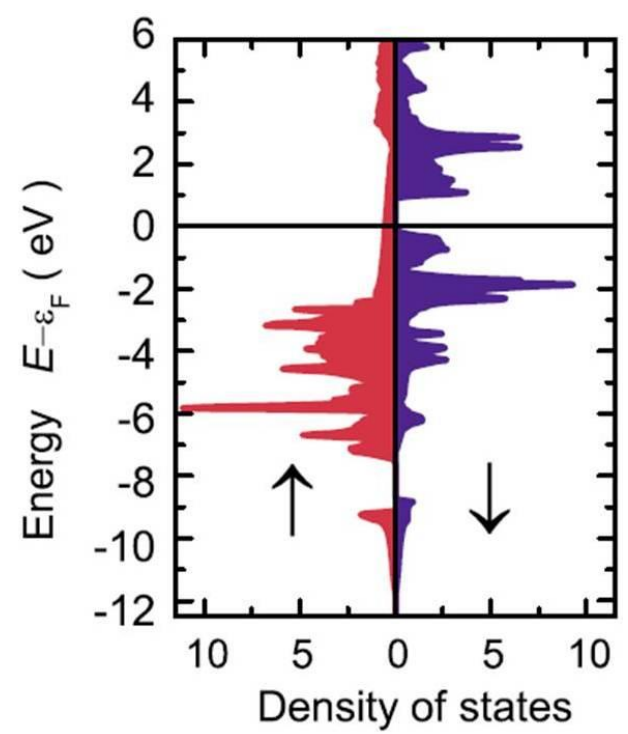

Fig. 2.1: Density of states of $\mathrm{Co}_{2} \mathrm{FeSi}$, one of $\mathrm{HMFs}$, for the majority and minority spin bands. The figure taken from [11]. 
For the device applications, HMFs have to satisfy three key requirements: large spin polarisation at $E_{\mathrm{F}}$, high $T_{\mathrm{C}}$ above $\mathrm{RT}$ and good interface quality. They are also preferred to have good conductance matching at interfaces between different layers (typically against a semiconductor) for better spin injection efficiency [12]. HMFs can fulfil these requirements due to their potential to exhibit $100 \%$ spin polarisation at $E_{\mathrm{F}}$.

There are four categories of materials that are theoretically predicted to be HMFs [6]; Heusler alloys (e.g., NiMnSb) [13], Zinc-blende compounds (e.g., CrAs and MnAs) [14], Perovskites (e.g., ( $\left.\mathrm{La}, \mathrm{Sr}) \mathrm{MnO}_{3}\right)$ [15] and oxide compounds (e.g., spinel $\mathrm{Fe}_{3} \mathrm{O}_{4}$ and rutile $\mathrm{CrO}_{2}$ ) [16].

Heusler alloys are selected in this study and hence are discussed in details in the following Section 2.2. The other three HMFs are discussed briefly using one example for each HMF. The half-metallicity of zinc-blende CrAs has been theoretically predicted based on the band structure calculations [13][17]. CrAs thin films have been epitaxially grown on $\mathrm{GaAs}(001)$ using MBE. It is found that CrAs is crystallised in the $B 3$ structure and shows $T_{\mathrm{C}}>400 \mathrm{~K}$, which is not sufficient for RT applications, with a magnetic moment of $3 \mu_{\mathrm{B}}$ /formula unit (f.u.) [18]. $\mathrm{LaSrMnO}_{3}$ (LSMO) is the most famous perovskite and has been theoretically predicted to be half-metallic [15]. $P$ of LSMO has been measured to be $78 \%$ and $72 \%$ using AR [7] and spin-polarised tunnelling [19], respectively. However, $P$ has been found to be decreased almost to zero near RT [20], which indicates that LSMO is not suitable for device applications at RT [20] although it has $T_{\mathrm{C}}=360 \mathrm{~K}$ [21]. $\mathrm{CrO}_{2}$ has $T_{\mathrm{C}}(=390 \mathrm{~K})$ [22][23], which again is not sufficient for RT applications because the gap shrinks at nonzero temperature. Its threshold temperature $\left(T^{*}\right)$ at which the band gap vanishes is less than $T_{\mathrm{C}}\left(T^{*}<300 \mathrm{~K}\right)$ [24]. Its magnetic moment is $2 \mu_{\mathrm{B}} /$ f.u. and it has been demonstrated that $\mathrm{CrO}_{2}$ has $P=96 \%$ using the AR technique [23]. 


\subsection{Heusler alloys}

Heusler alloys have been discovered in 1903 by Fritz Heusler [4][25]. The properties of the Heusler alloys can be different from those of the constituent elements [4]. For example, $\mathrm{Cu}_{2} \mathrm{MnAl}$ is ferromagnetic although none of the three elements (i.e., $\mathrm{Cu}, \mathrm{Mn}$ and $\mathrm{Al}$ ) are ferromagnetic independently [4]. The half-metallicity has first been discovered by de Groot et al. in 1983 in a Heusler alloy [4]. Since then, the Heusler alloys have attracted much interest owing to their potential applications in spintronics [4] due to their high $T_{\mathrm{C}}$ and large band-gap at $E_{\mathrm{F}}[6]$.

Heusler alloys have two different groups according to their crystalline structures; full- and half-Heusler alloys. The full-Heusler alloys crystallise in the $L 2_{1}$ structure with the form of $\mathrm{X}_{2} \mathrm{YZ}$ and the half-Heusler alloys crystallise in the $C 1_{b}$ structure with the form of $\mathrm{XYZ}$, where $\mathrm{X}$ and $\mathrm{Y}$ are transition metals (e.g., $\mathrm{Co}, \mathrm{Fe}, \mathrm{Ni}$ and $\mathrm{Mn}$ ); and $\mathrm{Z}$ is a semiconducting or non-magnetic metal (e.g., Al, Si, Ga, In and Sb) [6]. The $L 2_{1}$ structure consists of four interpenetrating face-centred cubic (fcc) sublattices occupied by $\mathrm{X}, \mathrm{Y}$ and $\mathrm{Z}$ atoms [6], while the $C 1_{b}$ structure consists of three interpenetrating fcc sublattices as depicted in Fig. 2.2(a) and (b). Hence, the half-Heusler alloys are considered to be less stable than the full-Heusler alloys and are fragile against atomic disorder because of the absence of the second $\mathrm{X}$ atom in their formula units [6][11]. The $L 2_{1}$ structure of the full-Heusler alloy can have atomic disorder when the $\mathrm{X}, \mathrm{Y}$ and $\mathrm{Z}$ atoms swap their locations randomly. There are two types of disorder: $B 2$ and $A 2$. If $\mathrm{Y}$ and $\mathrm{Z}$ atoms swap their locations ( $\mathrm{Y}-\mathrm{Z}$ disorder), the alloy turns into the $B 2$ structure [see Fig. 2.2(c)]. If $\mathrm{X}$ atoms replace their locations with $\mathrm{Y}(\mathrm{X}-\mathrm{Y}$ disorder) or with $\mathrm{Z}$ (X$\mathrm{Z}$ disorder), the alloy turns into the $A 2$ structure [see Fig. 2.2(d)]. 
a

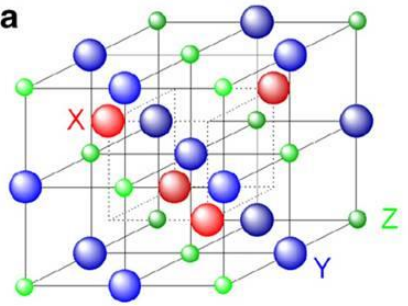

c

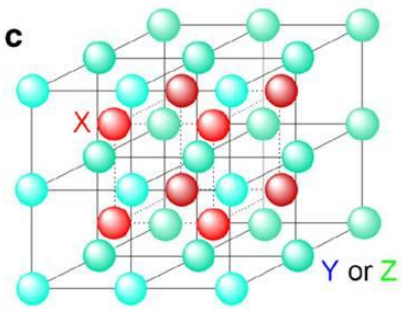

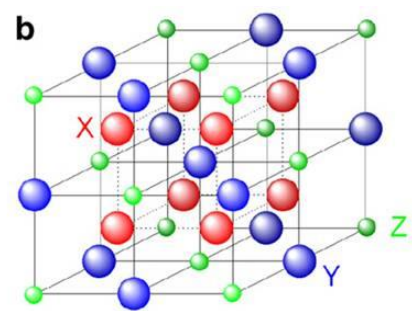

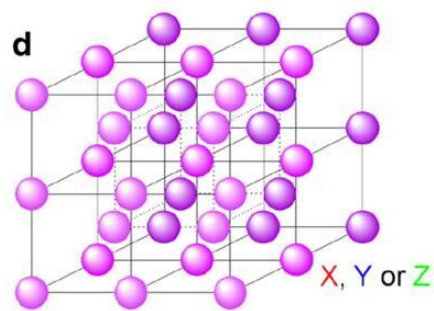

Fig. 2.2: Crystalline structures of two types of Heusler alloys; (a) half $\left(C 1_{b}\right)$ - and (b) full $\left(L 2_{1}\right)$-Heusler alloys. Disordered structures, (c) B2 and (d) A2 are also shown. The figure taken from [6].

There are more than 2400 Heusler alloys that can be formed by different combinations of the above elements from the periodic table [4][6]. Among them, Cobalt-based (Co-based) full-Heusler alloys have attracted much interest due to their high Curie temperature above RT (e.g., $T_{\mathrm{C}}$ for $\mathrm{Co}_{2} \mathrm{FeSi}$ is greater than $1100 \mathrm{~K}$ ) (see Fig. 2.3), good lattice constant matching with major single-crystal substrates (e.g., GaAs) (see Fig. 2.4) and the potential to engineer their spin density of states (DOS) near $E_{\mathrm{F}}$ by substituting constituent elements. In particular, all the Heusler alloys follow the generalised Slater-Pauling curve (see Section 2.2.2 for more details) according to the calculations of their DOS [4][6].

For instance, CFAS has very high $T_{\mathrm{C}}$ of $\sim 1100 \mathrm{~K}$ [26][27] and $P \sim 91 \%$ at RT estimated using a magnetic tunnel junction [28]. In addition to its large $P$, it exhibits the smallest temperature dependence in its spin polarisation. CFAS also possess a large band-gap with $E_{\mathrm{F}}$ in the middle of the minority gap [28], which makes it ideal for spintronic devices. Due to these excellent magnetic properties of CFAS and its family, CFS, these alloys have been selected for the band-gap measurements as described in details in Chapters 6 (CFS) and 7 (CFAS). 


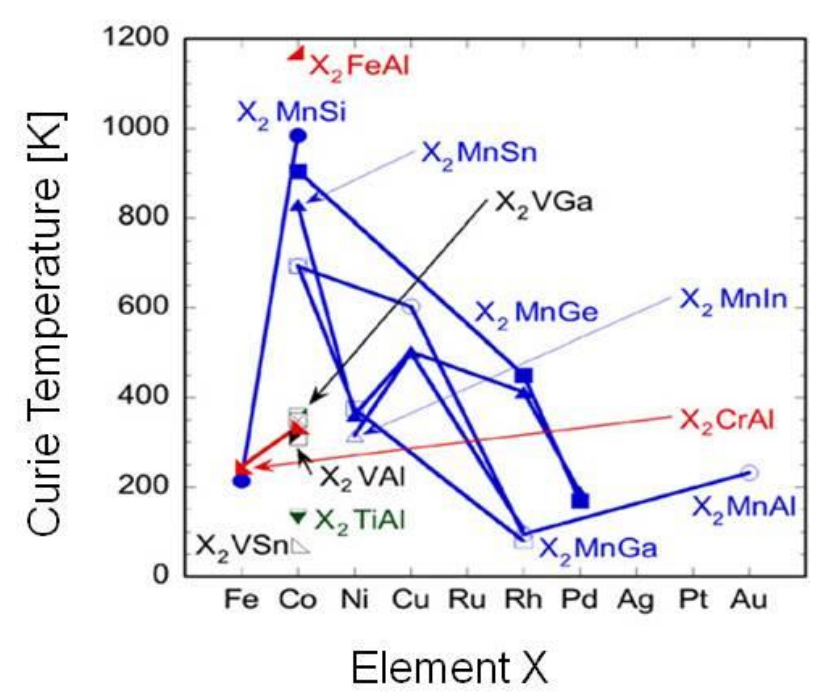

Fig. 2.3: Curie temperature distribution of full-Heusler bulk alloys with respect to the element $X$. The figure taken from [6].

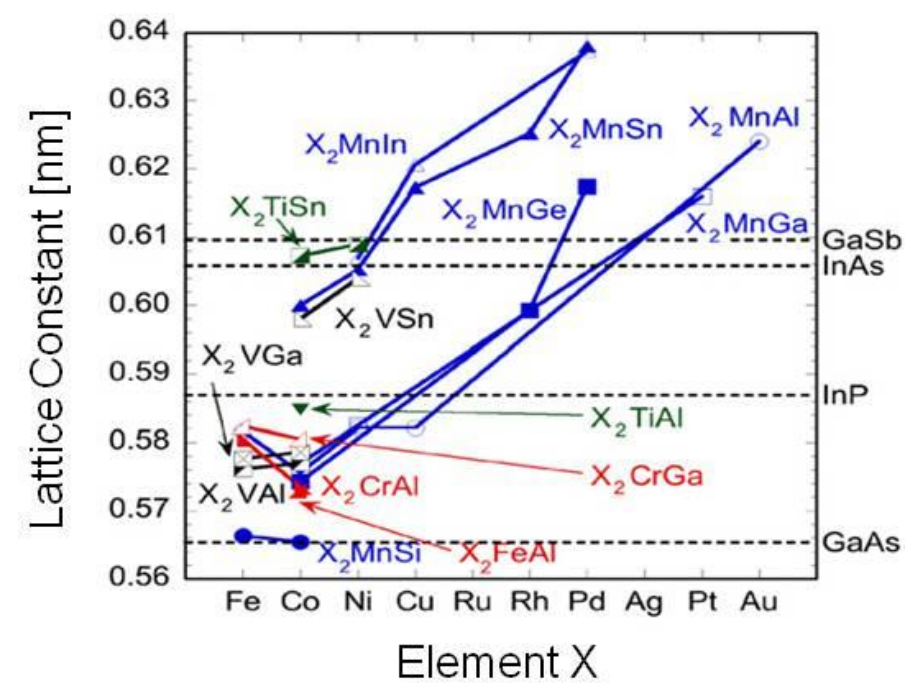

Fig. 2.4: Lattice constant distribution of full-Heusler bulk alloys with respect to the element $X$. The figure taken from [6].

\subsubsection{Origin of the band-gap in the full-Heusler alloys}

The elements of $\mathrm{X}, \mathrm{Y}$ and $\mathrm{Z}$ typically have spd-orbitals and the four sp-orbitals of these elements are located far below the Fermi level. Hence, the $s p$-orbitals do not contribute to the formation of a band-gap [29]. The band-gap in the minority spin band of the Heusler alloys originates predominantly from the strong covalent hybridisation between the two $d$-orbitals of the different transition metals (e.g., Co and Mn), following the 
hybridisation between the two X atoms (e.g., Co-Co) [29][30].

The $d$-orbitals are divided into five degenerate states; three-fold degenerate $\left(t_{2 g}\right)$ bonding states and two-fold degenerate $\left(e_{g}\right)$ antibonding states. The band-gap is formed between the higher $t_{2 g}$ bonding hybridised states in the valence band and the lower $e_{g}$ antibonding states in the conduction band of the Co-Co and the Mn $d$-orbitals as shown in Fig. 2.5. The antibonding $d$-orbitals of Co cannot couple with the $\mathrm{Mn} d$-orbitals, resulting a small band-gap near the Fermi level. This gap determines the band-gap of the full-Heusler alloys [29][30].

In the energy bands of the Co-Mn, all the five Co-Mn bonding orbitals are occupied, and all the five Co-Mn antibonding orbitals are empty. Hence, there are eight full and seven empty minority $d$-orbitals. Therefore, the maximum magnetic moment of the full-Heusler alloy is $7 \mu_{B} /$ f.u., if all the majority $d$-orbitals are occupied [29][30].

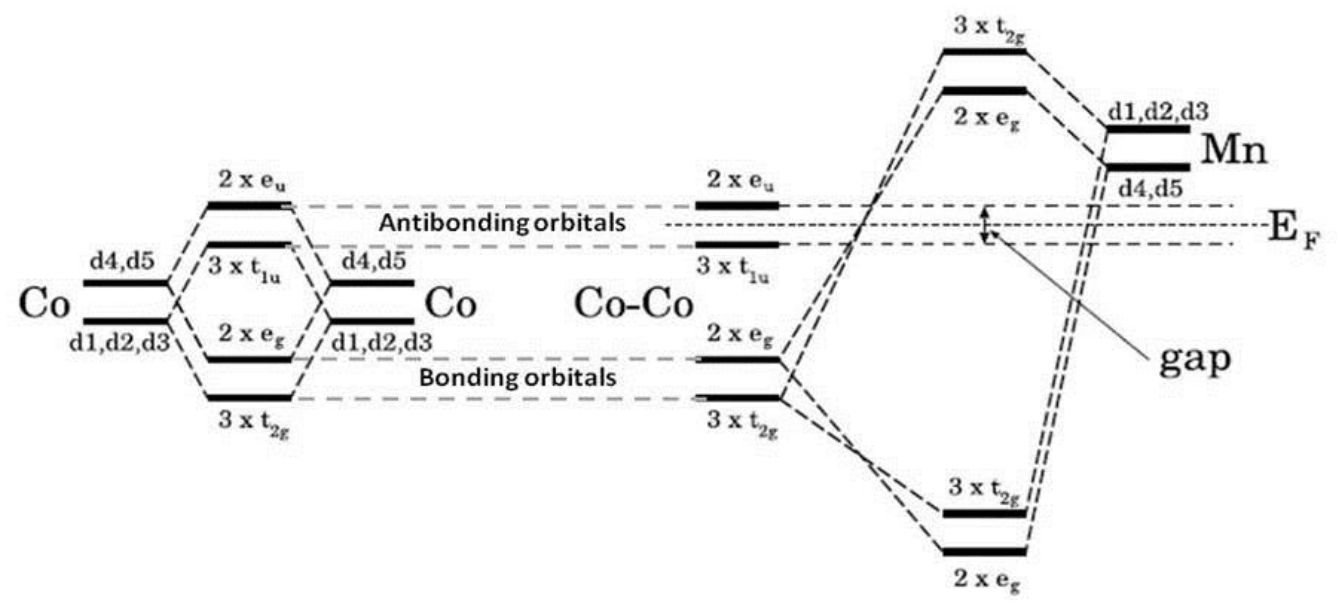

Fig. 2.5: Schematic illustration of the origin of the minority band-gap in full-Heusler alloys $\left(\mathrm{Co}_{2} \mathrm{MnZ}\right.$ as an example). The figure taken from [29].

\subsubsection{Generalised Slater-Pauling behaviour}

The total spin magnetic moment $\left(M_{\mathrm{t}}\right)$ is known to be proportional to the total number of valence electrons $\left(Z_{t}\right)$, which is called the Slater-Pauling behaviour [29][30]. This can be applied to the full-Heusler alloys as the generalised Slater-Pauling rule (see Fig. 2.6): 


$$
M_{\mathrm{t}}=Z_{\mathrm{t}}-24
$$

For the half-Heusler alloys, the rule can be modified as:

$$
M_{\mathrm{t}}=Z_{\mathrm{t}}-18
$$

The generalised Slater-Pauling behaviour is important as it allows to control the spin DOS at $E_{\mathrm{F}}$ by substituting the $\mathrm{X}, \mathrm{Y}$ and/or $\mathrm{Z}$ atoms with the other elements.

According to the theoretical calculations, however, only some of them can show the half-metallicity [6]. Their magnetic properties as a result of the change in the spin DOS are strongly influenced by atomic substitutions as discussed above. It is therefore possible for the Heusler alloys to be tailor-made to achieve desired magnetic properties.

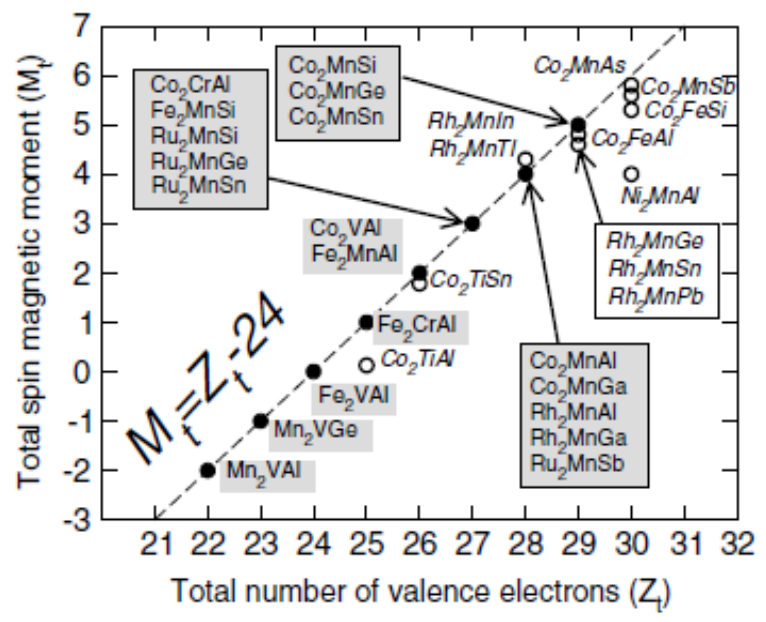

Fig. 2.6: Calculated total spin moments for full-Heusler alloys. The dashed line represents the generalised Slater-Pauling behaviour. The figure taken from [29].

\subsection{Reflectivity}

\subsubsection{Optical reflectance}

The interaction of a crystal with an electromagnetic field can be described by the dielectric function, $\epsilon(\omega, K)$ where $\omega$ is the frequency of the electromagnetic wave and $K$ the wave vector. The dielectric function has a sensitive relation with the electronic band structure of a crystal as explained below. Therefore, optical spectroscopy measurements are a useful instrument in determining the band structure of a crystal using the dielectric 
function. In the IR spectrum, the wavevector of the illumination is very small in comparison to the shortest reciprocal lattice vector. Therefore, the illumination wavevector is considered to be zero, allowing one to consider the dielectric function to be a function of the frequency only, which can be expressed as follows:

$$
\epsilon(\omega)=\epsilon^{\prime}(\omega)+\epsilon^{\prime \prime}(\omega)
$$

where $\epsilon^{\prime}(\omega)$ is the real part and $\epsilon^{\prime \prime}(\omega)$ is the imaginary part of the dielectric function [31]. The equivalent way of writing this is in terms of the refractive index $n(\omega)$ and the extinction coefficient $k(\omega)$,

$$
\epsilon(\omega)=(n-j k)^{2}
$$

meaning that $\epsilon^{\prime}(\omega)=n^{2}-k^{2}, \epsilon^{\prime \prime}(\omega)=-2 n k$

$r(\omega)$ is a complex function and can be defined as:

$$
r=\frac{\left|E^{r}\right|^{2}}{\left|E^{i}\right|^{2}}
$$

where $E^{i}$ and $E^{r}$ are the complex amplitudes of the incident and reflected light. In the case of normal incidence,

$$
r=\frac{|1-\kappa|^{2}}{|1+\kappa|^{2}}=\frac{(1-n)^{2}+k^{2}}{(1+n)^{2}+k^{2}}
$$

where $\kappa=\sqrt{\epsilon}$ is the complex refractive index so that $n=\operatorname{Re}(\kappa)$ and $k=\operatorname{Im}(\kappa)$ as in Eq. (2.4).

\subsubsection{Determination of $\epsilon(\omega)$ from the measured reflectivity}

In principle, it is possible to determine the entire spectrum of $n$ and $k$ from the reflectivity, however for this one needs a considerable amount of information which can be acquired by one of the two approaches as below: 
(1) Using Kramers-Kronig relations [32]:

$$
n(\omega)=1+P \int_{-\infty}^{\infty} \frac{d \omega^{\prime}}{\pi} \frac{k\left(\omega^{\prime}\right)}{\omega^{\prime}-\omega}, k(\omega)=-P \int_{-\infty}^{\infty} \frac{d \omega \prime}{\pi} \frac{n\left(\omega^{\prime}\right)-1}{\omega^{\prime}-\omega}
$$

by using one of these equations in addition to the data of $r(\omega)$ at all frequencies, one can in principle determine the values of $n(\omega)$ and $k(\omega)$. From the practical point of view, however, the numerical analysis can be complicated and require extra measurements to be conducted at different frequencies to provide reliable data to the entire frequency range as required by equation 2.6; it also requires very high measurement accuracy.

(2) Using the generalisation of equation 2.5 to non-normal angles of incidence [32]. This can provide the expressions for the reflectivity at an incidence angle, $\theta, n(\omega), k(\omega)$ and the polarisation of the incidence light. By combining these values with the measured reflectivity at an angle $\theta$, one can obtain the equations from which $n(\omega), k(\omega)$ can be extracted, the accuracy of measurement being again of paramount importance.

The purpose of this thesis however is measuring the band-gap of Heusler alloys, therefore the full restoration of $n(\omega), k(\omega)$ is not entirely necessary, our aim is recovering the band-gap value, as done for standard semiconductors, see for example [33]. This makes use of the fact that the function $k(\omega)$ varies sharply around the optical frequency corresponding to the band-gap $\omega=E_{g} / \hbar$. For direct-gap semiconductors, this sharp variation, within the first approximation, has the form of $k(\omega) \sim\left(\omega-E_{g} / \hbar\right)^{1 / 2}$; within the second approximation, taking into account Coulomb attraction between electrons and holes, the dependence is even sharper and resembles a step function [31]. For indirect gap semiconductors, within the first approximation, $k(\omega) \sim\left(\omega-E_{g} / \hbar\right)^{3 / 2}$ around the fundamental absorption edge.

As the Heusler alloy has some semiconducting properties and a band-gap at least for some electrons, it can be expected to have a fundamental absorption edge with the 
function $k(\omega)$ varying sharply at the frequency $\omega=E_{g} / \hbar$, although the exact nature of this dependence is unknown. It should be mentioned that, because of the KramersKronig relation, there is also some variation in $n(\omega)$ around the absorption edge but it is much more smooth because Kramers-Kronig relation is integral in nature, and sharp changes in " $k$ " can correspond to smooth changes in " $n$ ". However, due to the fact that " $k$ " has a steep change at the optical frequency or wavelength corresponding to the band-gap, this is enough to cause some variation in reflectance at that optical frequency according to formula (2.5b).

The difference between Heusler alloys and ordinary semiconductors [33] is that in Heusler alloys the band-gap is observed only in one spin direction, therefore to use reflectance to measure the band-gap of Heusler alloys, therefore the measurements require application of magnetic field and differential techniques as described below.

\subsection{Magnetoresistive effects}

\subsubsection{Tunnelling magnetoresistance (TMR)}

A MTJ consists of a thin tunnelling barrier (insulating layer) sandwiched by two ferromagnetic (FM) electrodes. Its resistance depends on the relative magnetisation orientations (parallel or antiparallel) of the two FM layers [34-36]. During the tunnelling process, only spin-up and spin-down electrons from one FM can tunnel into the same spin states in another FM. As the magnetisation configuration of the two electrodes changes from parallel to antiparallel, less spin-polarised electrons can tunnel as shown in Fig. 2.7. The tunnelling resistance $(R)$ of the parallel configuration $\left(R_{\mathrm{P}}\right)$ is hence lower than that of the antiparallel configuration $\left(R_{\mathrm{AP}}\right)$. The change in the resistance is called tunnelling magnetoresistance (TMR) [34-36]. The TMR ratio can be defined by the Julliere formula [37]: 


$$
\text { TMR ratio }=\frac{2 P_{1} P_{2}}{1-P_{1} P_{2}}
$$

where $P_{1}$ and $P_{2}$ are the spin polarisation of the two FM electrodes 1 and 2, respectively.
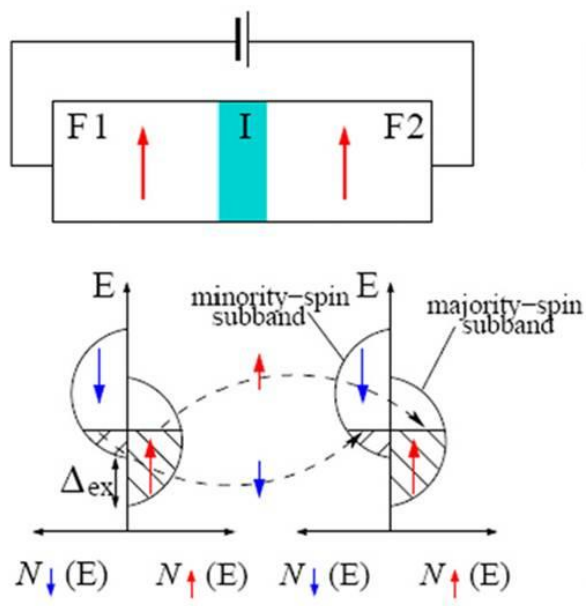

(a)
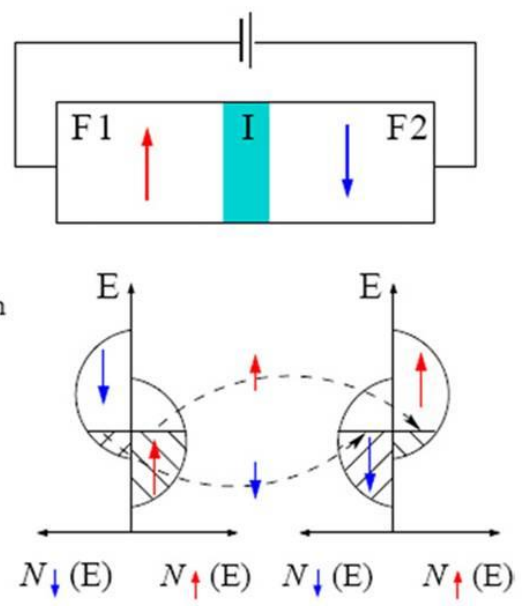

(b)

Fig. 2.7: Schematic diagrams of an MTJ and the corresponding TMR effect for (a) parallel and (b) antiparallel configurations. The figure taken from [38].

In 1975, the first TMR effect was observed by Julliere, showing a TMR ratio of about $14 \%$ in $\mathrm{Fe} / \mathrm{Ge} / \mathrm{Co}$ junctions at $4 \mathrm{~K}$ [37]. Twenty years later, an amorphous $\mathrm{AlO}_{x}$ barrier was used in MTJs, and the TMR effect was observed at RT with the TMR ratios of $18 \%$ and $11.8 \%$ for $\mathrm{Fe} / \mathrm{Al}_{2} \mathrm{O}_{3} / \mathrm{Fe}$ [39] and $\mathrm{CoFe} / \mathrm{Al}_{2} \mathrm{O}_{3} / \mathrm{Co}$ junctions [40], respectively. Since then, $\mathrm{AlO}_{x}$ barriers in the MTJs have been investigated by many groups to improve their properties in combination with search for FM materials with larger spin polarisation to exhibit larger TMR ratios. In 2004, a TMR ratio as high as $70 \%$ was reported for $\mathrm{CoFeB} / \mathrm{AlO}_{x} / \mathrm{CoFeB}$ junctions with the spin polarisation of $61 \%$ for the CoFeB electrodes as estimated using Eq. (2.7) [37]. Researchers also conducted many theoretical calculations and predicted even larger TMR ratios for $\mathrm{Fe} / \mathrm{MgO} / \mathrm{Fe}$ MTJs, where $\mathrm{MgO}$ was a single crystal with the (001) surface orientation [41][42]. The use of $\mathrm{MgO}$ boosted the TMR ratios experimentally to be over 100\% [43]. 
To date, the TMR ratio has been exceeded $600 \%$ in MTJ consisting of $\mathrm{CoFeB} / \mathrm{MgO} / \mathrm{CoFeB}$ (001)-oriented layers at RT [44]. Recently, researchers have been studying on MTJs with the FM magnetisations perpendicular to plane. Such a perpendicularly magnetised MTJ can be used as read heads of HDD and memory cells of MRAM for higher recording density [see Section 2.5 for details].

\subsubsection{Giant magnetoresistance (GMR)}

GMR effect was discovered independently by Grünberg et al. and Fert et al. in 1988 [38][45]. This discovery led to extensive experimental and theoretical works due to the applicability to devices (e.g., read heads of HDDs and magnetic sensors). The GMR effect was observed first in a multilayered structure consisting of FM and non-magnetic (NM) layers of $\mathrm{Fe}$ and $\mathrm{Cr}$, respectively [45]. Currently, a GMR structure typically consists of a FM/NM/FM spin-valve trilayer [3], where one FM layer is pinned and another FM layer is free to be reversed by an external magnetic field.

The GMR effect is similar to the TMR effect as described in Section 2.4.1, in which the electrical resistance depends on the configuration of the FM layers, either parallel or antiparallel [see Fig. 2.8(a)]. For the parallel (P) configuration, the resistance is lower than that for the antiparallel (AP) configuration due to the spin-polarised electron scattering. These configurations can be achieved by applying a magnetic field. Therefore, a GMR ratio can be calculated using the following formula:

$$
\frac{\Delta R}{R}=\frac{R_{\mathrm{max}}-R_{\min }}{R_{\min }}
$$

where $R_{\max }$ is the maximum resistance in the antiparallel configuration and $R_{\min }$ is the minimum resistance in the parallel configuration [3]. 


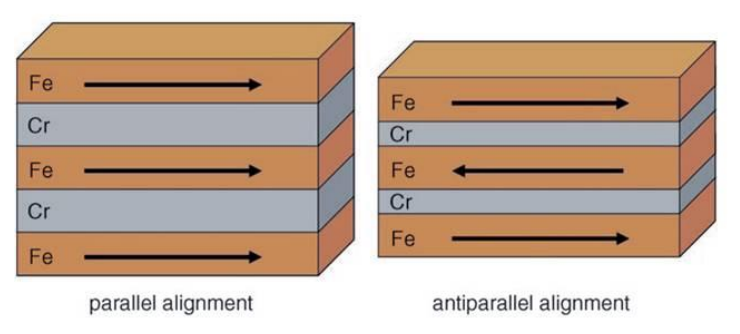

(a)

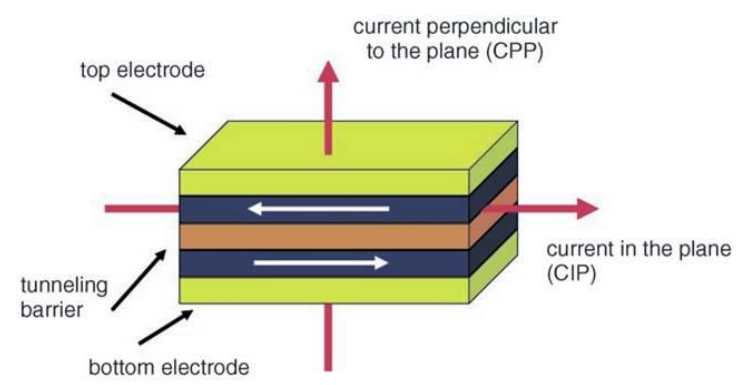

(b)

Fig. 2.8: (a) Schematic diagram of a multilayer of $\mathrm{Fe}$ and $\mathrm{Cr}$ with parallel (left) and antiparallel (right) magnetic configurations. (b) Basic setup for the GMR measurements. The directions of the sensing currents for the CPP- and CIP-GMR measurements are shown by arrows. The figure taken from [5].

There are two measurement configurations to detect the GMR effect [see Fig. 2.8 (b)]; current in the plane (CIP) [45][46] and current perpendicular to the plane (CPP) [47]. The CIP configuration has been used during the first few years since the GMR discovery [48]. In the CIP configuration, a sensing current is applied in the plane of the multilayer, which leads a spin-polarised current passing through the surface few layers (i.e., $5 \mathrm{~nm}$ ) [49] of the multilayers predominantly, leading to interfacial scattering at the ferromagnetic layer surfaces with the opposite spin polarisation to the electrons polarised by the top-most ferromagnetic layer. For the CPP configuration, a current is applied perpendicular to the multilayer, which leads to a larger effect due to the more effective spin scattering at the ferromagnet/non-magnet interfaces. CPP-GMR has several advantages over CIP-GMR, such as longer critical length (i.e., spin diffusion length with typically between 0.1 and $1 \mu \mathrm{m}$ for $\mathrm{NiCr} / \mathrm{Cu} / \mathrm{Co} / \mathrm{Cu}$ multilayered structure) [50]. Spin diffusion length can be defined as the mean distance of which electrons travel with their initial spin polarisation before they spin-flipping (i.e., change the spin direction) [51][52]. It therefore supports a multilayer of relatively thick layers (e.g., nanopillar structures). The quantitative analysis of the GMR effect can be obtained in the CPP measurements. It should be noted that the CPP measurements are less sensitive to surfaces roughness of the multilayers [47]. Hence, CPP is the preferred configuration 
and it was used in the spintronic devices (e.g., spin-valve read heads and MRAM).

\subsection{Spintronic devices}

The manipulation of spin-polarised electrons provides further development in the performance of spintronic devices including miniaturisation of the devices, increase in the operation speed and decrease in the power consumption [3]. Several spintronic devices have already been released in markets and more devices have been under development. Here, two examples of these devices are described below.

\subsubsection{Magnetic random access memory (MRAM)}

MRAM is one of the most promising new spintronic devices [3]. It is expected to be a new universal memory and therefore to replace the current HDD and dynamic read access memory (DRAM) [3]. MRAM has several advantages, such as (i) non-volatility, in which the information is retained when the power is switched-off, (ii) high speed, in which reading and writing data is in the order of nano-seconds, (iii) high storage density

(> $1 \mathrm{Gbit} / \mathrm{in}^{2}$ in the latest MRAM) [3] and (iv) low-power consumption (120 pJ to read and write data) [3].

Although MRAM concept was first proposed by Schwee in 1972, the demonstrations of MRAM took place at the end of the 1990s when 1 kbit and $512 \mathrm{kbit}$ MRAM was developed by IBM and Motorola, respectively [3]. Since then, there has been significant improvement in the storage capacity of up to $64 \mathrm{Mbit}$, which has been demonstrated by Toshiba [53] and commercialised by EverSpin [54].

MRAM basically consists of a MTJ (or a spin-valve for early MRAMs) cell to store data and a complementary metal-oxide-semiconductor (CMOS) transistor to read and write information (see Fig. 2.9). In MRAM, data bits are not stored as an electric charge as in the conventional DRAM but they are stored as a magnetisation state in a 
MTJ (or a spin-valve) element. Using the relative magnetisations between free and pinned magnetic layers, the logical " 0 " and " 1 " data can be stored. To read the data, the TMR (or GMR) effect can be used.

A MTJ element is connected to bit and write (digit) lines from its top and bottom sides, respectively. These wires are aligned perpendicular to each other. To write data in MRAM, writing currents are applied to the bit and write lines. These currents generate a magnetic field and change the magnetisation of the MTJ free layer.

To read data, the magnetoresistance effect is employed. If the free and pinned layers in MTJ (or spin-valve) have the AP configuration, a small current flows due to the high resistance, corresponding to the "1" state in the cell. Conversely, if MTJ (or spin-valve) has the P configuration, a large current flows through MTJ (or spin-valve) due to the low resistance, indicating the " 0 " state.

There are several challenges in the MRAM operation and design. For instance, during the data writing, a generated magnetic field may interfere and change the magnetisations of the neighbouring cells, which causes errors. Additionally, the field for writing increases with decreasing the cell size. Implementing a transistor for each cell limits the high-density MRAM and miniaturisation. Therefore, one-transistor (1T) with four MRAM cells (4MTJ) was proposed to achieve a high-density memory [3]. In addition, there are also the other obstacles in the MRAM operation such as a reference voltage to operate the MRAM and thermal stability $\left(k_{\mathrm{B}} T>70\right)[3][55]$.

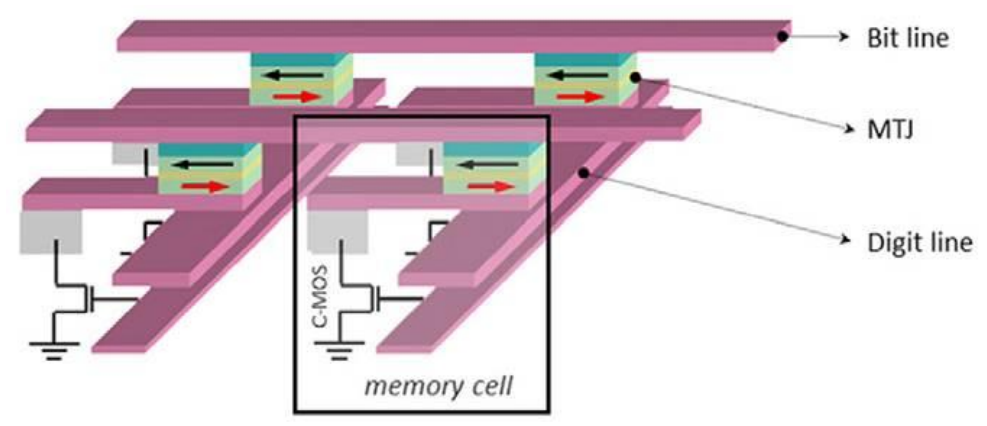

Fig. 2.9: Schematic diagram of MRAM. The figure taken from [3]. 
Recently, spin-transfer torque MRAM (STT-MRAM) has been proposed [3][56] and has been demonstrated experimentally [3]. The advantages of using STT are as follows: (i) It enables the spin-polarised current to transfer its spin angular momentum to the free layer and switches the magnetisation for the individual cells [56]. (ii) The required writing current is reduced due to the scaling in a nanometre-size cell. For instance, 2 Mbit STT-MRAM has been demonstrated by Hitachi in collaboration with Tohoku University in 2007 [53][57]. STT-MRAM technology can lead to a revolution in the memories and the associated electronic markets [53].

\subsubsection{Spin-polarised field effect transistor (spin-FET)}

A spin-FET has been first proposed by Datta and Das in 1990 [58]. Spin-FET consists of FM source and drain, which are used to inject and detect a spin-polarised current, separated by a NM metal or two-dimensional electron gas (2DEG), which provides long spin lifetime. The gate length should be designed to be less than the spin diffusion length [58].

FMs can be used to inject a spin-polarised current into semiconductors (SC). However, the FM/SC structures have a conductivity mismatch and that decreases the efficiency of the injection of spin-polarised carriers [12]. In order to avoid the conductivity mismatch, three methods have been proposed. The first method is to use HMFs with $100 \%$ spin polarisation. The second one is to use materials with similar conductivity with SC [e.g., dilute magnetic semiconductors (DMS)][6]. The third is to inject spins through a tunnel barrier (e.g., $\mathrm{MgO})$. 


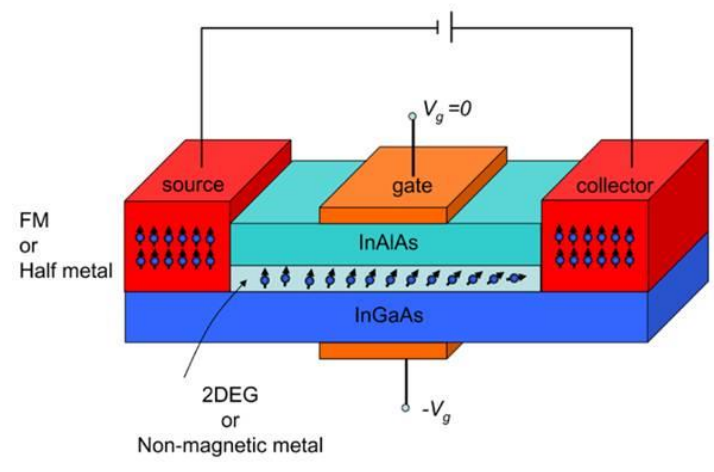

(a)

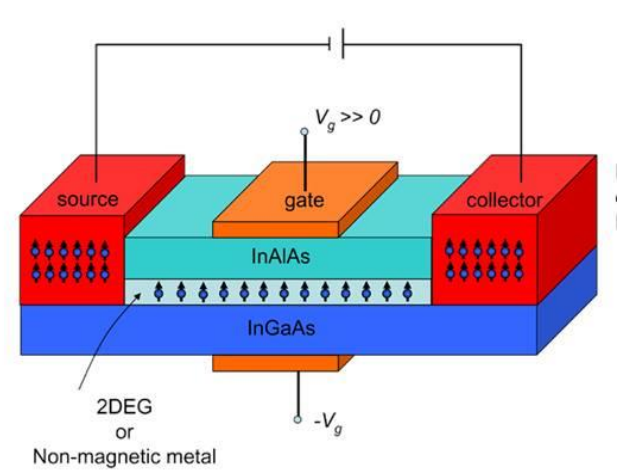

(b)

Fig. 2.10: Schematic diagram of spin-FET with the (a) on and (b) off states. The figure taken from [59].

If there is no voltage applied at the gate, a spin-polarised current can flow with maintaining the same spin-orientation from the source to the drain through the channel. The magnetoresistive signals become low for the magnetisations of the two FM layers which are in parallel and therefore the spin-current flow through the channel. In this case, the spin-FET is turned on [see Fig. 2.10(a)]. In the case of applying a voltage at the gate, the spin-orientation of the current is changed. This change is proportional to the applied voltage and thus preventing the spin current to flow and the spin-FET is turned off [see Fig. 2.10(b)]. Therefore, the spin-FET operates similar to the conventional FET, where the resistance is changed by the gate voltage. However, the spin-FET operation can also arise from the change in the spin orientation by the gate, which can be detected by changing the detector magnetisation [60]. This provides multiple states for the operation. 


\section{Chapter 3}

\section{Experimental Set-up}

A new experimental set-up was designed to measure the band-gap of half-metallic ferromagnets at RT and at low temperature using a cryostat with optical windows. The set-up was based on spectroscopic measurement techniques with a range of photon energy that has been widely used to measure semiconductor band-gaps. Two measurement configurations were selected for reflection and transmission modes. Here the reflection mode was used due to its simplicity as compared to the transmission method [61].

\subsection{Criteria of the experimental set-up}

This experimental set-up requires eight criteria to satisfy as follows: (i) The wavelength has to cover an expected band-gap energy range of the Heusler alloy films. (ii) Pulsed light needs to be used to excite the minority spin electrons. (iii) Circularly-polarised light needs to be generated. (iv) A photodetector has to cover the wavelength range, corresponding to the band-gap energy range to be measured with high sensitivity. (v) Optical matching needs to be achieved in the wavelength range of all components. (vi) The received signals from the photodetector have to be locked onto a reference signal 
that took from the output of the optical chopper. (vii) A sample holder needs to be made with a simple design for better controllability of the films and the magnet. (viii) The light travelling distance must be minimised in order to keep the signal intensities as high as possible.

\subsubsection{Wavelength}

As we can see from Table 3.1, the band-gaps of major Heusler alloy films were calculated theoretically and measured experimentally. The band-gaps were found to be between $\sim 0.062$ and $0.62 \mathrm{eV}[6][61]$. This range of band-gap energies needs to be converted into the corresponding wavelengths by using the following "conversion" formula:

$$
E=\frac{h c}{\lambda}
$$

where $E$ is the energy of photon in Joule $(\mathrm{J}), h$ is Planck's constant $\left(6.626 \times 10^{-34} \mathrm{~J} . \mathrm{s}\right), c$ is the speed of light $\left(2.998 \times 10^{8} \mathrm{~m} / \mathrm{s}\right)$ and $\lambda$ is the wavelength in meter $(\mathrm{m})$. By multiplying the constants $h$ and $c$, the resultant is $19.865 \times 10^{-26} \mathrm{~J} \cdot \mathrm{m}$. However, the common units for photon energy in electron volt $(\mathrm{eV})$, where $1 \mathrm{eV}=1.602 \times 10^{-19} \mathrm{~J}$. Therefore, the resultant can be converted into units of $\mathrm{eV}$ as follows:

$h c=19.865 \times 10^{-26} / 1.602 \times 10^{-19}=1.24 \times 10^{-6} \mathrm{eV}$.

The whole formula can be expressed in terms of $\mathrm{eV}$ and $\mu \mathrm{m}$ as follow:

$$
E(\mathrm{eV})=\frac{1.24}{\lambda(\mu m)}
$$

By applying this formula, the wavelength was found to be in the range between 2 to $20 \mu \mathrm{m}($ e.g., $E(\mathrm{eV})=1.24 \mu \mathrm{m} / 2 \mu \mathrm{m}=0.62 \mathrm{eV})$, which fell in the mid-infrared (IR) range. Hence, the IR source was an essential part in this set-up. 
There are different types of IR light sources to choose from, such as lasers, halogen lamps and ceramic elements. Each type has their advantages and disadvantages. Lasers, for instance, have collimated light that make it easy to control the light direction, high power output and easy operation ability. However, they mostly are bulky and expensive with narrow wavelength spectra. Hence, they have been excluded from this study. Halogen lamps and ceramic elements are good alternatives due to their broad wavelength spectra with relative high power. They have much smaller element size and are inexpensive, even though they require more effort to focus the light to a sample.

In this study, the choice was an IR lamp (LOT-Oriel, LSB150) due to its wide wavelength coverage (between 1 and $24 \mu \mathrm{m}$ ) and its high emissivity (> 60\%). This wavelength coverage corresponds to the photon energy $(h v)$ between 1.24 and $0.052 \mathrm{eV}$, matching $E_{\mathrm{g}}$ of the Heusler thin films as discussed above. This IR lamp consists of a coil made of an oxidation-resistant wire to emit IR light at high temperature. It has a power of $10 \mathrm{~W}$ and the emissivity profile is shown in Fig. 3.1 (dotted curve). 
Table 3.1: List of magnetic properties of Heusler alloys studied in this thesis. More materials can be found in Ref. 6 .

\begin{tabular}{|c|c|c|c|c|c|c|c|}
\hline \multirow{2}{*}{ 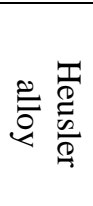 } & \multicolumn{2}{|c|}{$\begin{array}{l}\text { Magnetic moment }(m) \text { in } \\
\text { formula unit }\left[\boldsymbol{\mu}_{\mathrm{B}} / \mathbf{f} \text {.u. }\right]\end{array}$} & \multicolumn{2}{|c|}{$\begin{array}{l}\text { Saturation magnetisation } \\
\left(M_{\mathrm{s}}\right)\left[\mathbf{e m u} / \mathbf{c m}^{3}\right] / \mu_{\mathrm{B}} / \mathbf{f} . \mathbf{u} .\end{array}$} & \multirow{2}{*}{ 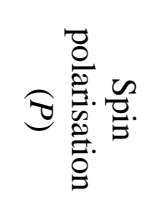 } & \multirow{2}{*}{ 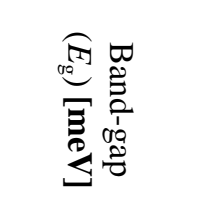 } & \multirow{2}{*}{ 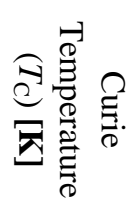 } \\
\hline & Calculation & Experiment & Calculation & Experiment & & & \\
\hline \multirow{5}{*}{$\begin{array}{l}0 \\
0 \\
01 \\
0 \\
0\end{array}$} & $\begin{array}{c}6 \\
\text { (at 0K) } \\
{[62][63]}\end{array}$ & $\begin{array}{c}5.97 \pm 0.05 \\
\text { (at 5K) } \\
{[62][63]}\end{array}$ & $\begin{array}{c}5.7-6 \\
\mu_{\mathrm{B}} / \text { f.u. } \\
{[70]}\end{array}$ & $\begin{array}{c}\sim 860 / 4.2 \\
\quad[68]\end{array}$ & $\begin{array}{c}49 \pm 2 \% \\
(\mathrm{PCAR}) \text { at } \\
5 \mathrm{~K} \\
{[64]}\end{array}$ & $\begin{array}{c}100 \\
\text { (DFT) } \\
{[65]}\end{array}$ & $\begin{array}{c}1100 \pm 20 \\
{[4]}\end{array}$ \\
\hline & & & & $\begin{array}{c}1130 / \\
5.4919 \\
{[69]} \\
\end{array}$ & $\begin{array}{c}\sim 58 \% \\
(\mathrm{GGA}) \\
{[66]} \\
\end{array}$ & $\begin{array}{c}146 \text { (GGA) } \\
{[66]}\end{array}$ & $\begin{array}{c}1185 \\
{[4]}\end{array}$ \\
\hline & & & & & $\begin{array}{c}\text { 50\% } \\
\text { (MTJ) } \\
{[64]}\end{array}$ & $\begin{array}{c}400 \\
(\mathrm{LDA})[67]\end{array}$ & \\
\hline & & & & & $\begin{array}{c}\sim 61 \% \\
(\mathrm{MTJ}) \\
\text { at RT } \\
{[18]}\end{array}$ & $\begin{array}{l}\quad 780 \\
(\mathrm{LDA}+U) \\
{[14][66]}\end{array}$ & \\
\hline & & & & & $\begin{array}{c}\sim 93 \% \\
(\mathrm{LDA}+U) \\
{[66]}\end{array}$ & & \\
\hline \multirow{8}{*}{ 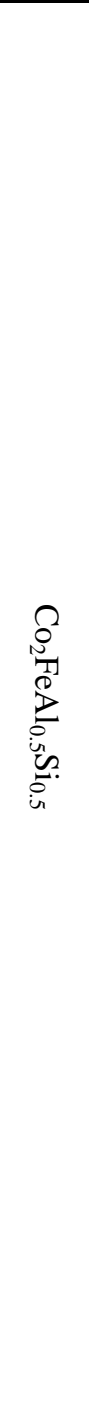 } & $\begin{array}{l}5.5 \mu_{\mathrm{B}} / \text { f.u. } \\
{[70][71]}\end{array}$ & & & $\begin{array}{c}5.61 \mu_{\mathrm{B}} / \text { f.u. } \\
{[70]}\end{array}$ & $\begin{array}{c}91 \% \\
(\mathrm{MTJ}) \\
\text { at RT } \\
{[28]}\end{array}$ & $\begin{array}{l}600(\mathrm{MTJ}) \text { at } \\
7 \mathrm{~K}[73]\end{array}$ & $\begin{array}{c}1150 \pm 50 \\
{[27]}\end{array}$ \\
\hline & & & & $\begin{array}{c}5.33 \pm 0.135 \\
\mu_{\mathrm{B}} / \text { f.u. } \\
{[6]}\end{array}$ & $\begin{array}{c}70 \% \\
(\mathrm{MTJ}) \\
\text { at } \mathrm{RT}, \text { ann. } \\
\text { at } 400^{\circ} \mathrm{C} \\
{[72]}\end{array}$ & $\begin{array}{c}760 \\
(\mathrm{LDA}+U) \\
{[70][75-} \\
77][79]\end{array}$ & $\begin{array}{c}1100 \\
\text { (bulk) } \\
{[26]}\end{array}$ \\
\hline & & & & $\begin{array}{c}880 \mathrm{emu} / \mathrm{cm}^{3} \\
{[80]}\end{array}$ & $\begin{array}{c}56 \% \\
(\mathrm{MTJ}) \\
\text { at } \mathrm{RT}, \text { ann. } \\
\text { at } 600^{\circ} \mathrm{C} \\
{[72]}\end{array}$ & $\begin{array}{c}1200 \\
\text { (bias voltage } \\
\text { dependence } \\
\text { of } d I / d V \text { ) at } \\
7 \mathrm{~K} \\
{[73]}\end{array}$ & \\
\hline & & & & $\begin{array}{c}900 \mathrm{emu} / \mathrm{cm}^{3} \\
{[74]}\end{array}$ & $\begin{array}{c}60 \pm 1 \% \\
\text { (PCAR) } \\
\text { at } 5 \mathrm{~K} \\
\text { (bulk) }[71]\end{array}$ & & \\
\hline & & & & $\begin{array}{c}\sim 900 \\
\mathrm{emu} / \mathrm{cm}^{3} \\
{[79]}\end{array}$ & $\begin{array}{c}68 \% \\
(\mathrm{MTJ}) \\
\text { at RT } \\
{[76]}\end{array}$ & & \\
\hline & & & & $\begin{array}{c}1100 \\
\mathrm{emu} / \mathrm{cm}^{3} \\
{[81]}\end{array}$ & $\begin{array}{c}71 \% \\
(\mathrm{MTJ}) \\
\text { at } 7 \mathrm{~K} \\
{[74]}\end{array}$ & & \\
\hline & & & & $\begin{array}{c}1100 \\
\mathrm{emu} / \mathrm{cm}^{3} \\
{[82]}\end{array}$ & $\begin{array}{c}81 \% \\
(\mathrm{MTJ}) \\
\text { at 5K } \\
{[73]}\end{array}$ & & \\
\hline & & & & & $\begin{array}{c}81 \% \\
(\mathrm{MTJ}) \\
\text { at RT } \\
{[14]}\end{array}$ & & \\
\hline
\end{tabular}




\begin{tabular}{|c|c|c|c|c|c|c|}
\hline \multirow{10}{*}{$\begin{array}{l}z \\
z \\
z \\
z \\
\sigma\end{array}$} & $\begin{array}{c}3.94 \mu_{\mathrm{B}} / \text { f.u. } \\
{[91]}\end{array}$ & $\begin{array}{c}4 \mu_{\mathrm{B}} / \text { f.u. } \\
\text { (bulk and } \\
\text { film) } \\
\text { at } 5 \mathrm{~K} \\
{[12]} \\
\end{array}$ & $\begin{array}{c}3.6 \mu_{\mathrm{B}} / \text { f.u. } \\
\text { (film) } \\
\text { at } 4 \mathrm{~K} \\
{[84]}\end{array}$ & $\begin{array}{c}50 \% \\
- \text { bulk } \\
\text { (photoem } \\
\text { ission) } \\
{[95]}\end{array}$ & $\begin{array}{c}\sim 500 \\
\text { (theoretically } \\
\text { predicted) } \\
{[13][86]} \\
{[96][102]}\end{array}$ & $\begin{array}{c}730 \\
{[24]} \\
{[83-85]} \\
{[88-90]} \\
{[99]} \\
\end{array}$ \\
\hline & & $\begin{array}{c}3.6 \mu_{\mathrm{B}} / \mathrm{f} . u . \\
(\text { film) } \\
\text { at } 4 \mathrm{~K} \\
{[5]}\end{array}$ & $\begin{array}{c}\sim 538 \\
\mathrm{emu}^{2} / \mathrm{cm}^{3} \\
(\text { film) } \\
\text { at 5K } \\
{[97]}\end{array}$ & $\begin{array}{c}35-50 \% \\
- \text { bulk } \\
\text { (PCAR) } \\
\text { at } 1.5 \mathrm{~K} \\
\text { [87] }\end{array}$ & $\begin{array}{c}168 \\
\text { (first- } \\
\text { principles } \\
\text { calculations) } \\
{[100]}\end{array}$ & $\begin{array}{c}728 \\
{[86][94]}\end{array}$ \\
\hline & & $\begin{array}{c}3.9 \pm 0.2 \\
\text { (film) } \\
{[83]}\end{array}$ & $\begin{array}{c}4.0 \pm 0.06 \\
\mu_{\mathrm{B}} / \mathrm{f} . u . \\
\text { (film) } \\
\text { at } 5 \mathrm{~K} \\
{[89]}\end{array}$ & $\begin{array}{c}\sim 44 \%- \\
\text { bulk } \\
\text { (PCAR) } \\
\text { at } 4.2 \mathrm{~K} \\
{[94]}\end{array}$ & $\begin{array}{c}540 \\
(\mathrm{DFT}) \\
{[103]}\end{array}$ & $\begin{array}{l}756 \\
{[92]}\end{array}$ \\
\hline & & $\begin{array}{c}3.5 \mu_{\mathrm{B}} / \text { f.u. } \\
\text { (film) } \\
\text { at } 5 \mathrm{~K} \\
{[24]}\end{array}$ & & $\begin{array}{c}\sim 45 \%- \\
\text { bulk } \\
\text { (PCAR) } \\
\text { at } 4.2 \mathrm{~K} \\
{[85][86]}\end{array}$ & $\begin{array}{c}\sim 400 \\
\text { (measured by } \\
\text { IR absorption } \\
\text { at RT) } \\
{[101]} \\
\end{array}$ & \\
\hline & & $\begin{array}{c}4 \mu_{\mathrm{B}} / \text { f.u. } \\
\text { (film) } \\
\text { at } 5 \mathrm{~K} \\
{[24]}\end{array}$ & & $\begin{array}{c}58 \pm 2.3 \% \\
(\mathrm{PCAR}) \\
{[7][71]} \\
{[95]}\end{array}$ & & \\
\hline & & $\begin{array}{c}4 \pm 0.02 \\
\mu_{\mathrm{B}} / \text { /f.u. } \\
\text { (bulk) } \\
\text { at } 5 \mathrm{~K} \\
{[85]}\end{array}$ & & $\begin{array}{c}20-40 \% \\
\text { (Spin- } \\
\text { resolved } \\
\text { photoemis } \\
\text { sion, } \\
\text { SRPES)- } \\
\text { film [96] } \\
\end{array}$ & & \\
\hline & & $\begin{array}{c}4.02 \mu_{\mathrm{B}} / \text { f.u. } \\
\text { (bulk) } \\
\text { at } 5 \mathrm{~K} \\
{[86]}\end{array}$ & & $\begin{array}{c}28 \pm 2 \% \\
\text { (spin- } \\
\text { polarised } \\
\text { tunneling } \\
\text { technique) } \\
\text { - film at } \\
0.4 \mathrm{~K} \\
{[63]}\end{array}$ & & \\
\hline & & & & $\begin{array}{c}\sim 100 \% \\
(100+0 \\
/-12 \%) \\
\text { (spin- } \\
\text { polarized } \\
\text { inverse } \\
\text { photoemis } \\
\text { sion, } \\
\text { SPIP)- } \\
\text { film at } \\
200 \mathrm{~K} \\
{[93]} \\
\end{array}$ & & \\
\hline & & & & $\begin{array}{c}99.3 \\
\text { (Calculate } \\
\text { d- DFT } \\
\text { within } \\
\text { LDA) } \\
\text { [97] }\end{array}$ & & \\
\hline & & & & $\begin{array}{c}99 \\
\text { (Calculate } \\
\text { d- first } \\
\text { principle) } \\
{[98]} \\
\end{array}$ & & \\
\hline
\end{tabular}




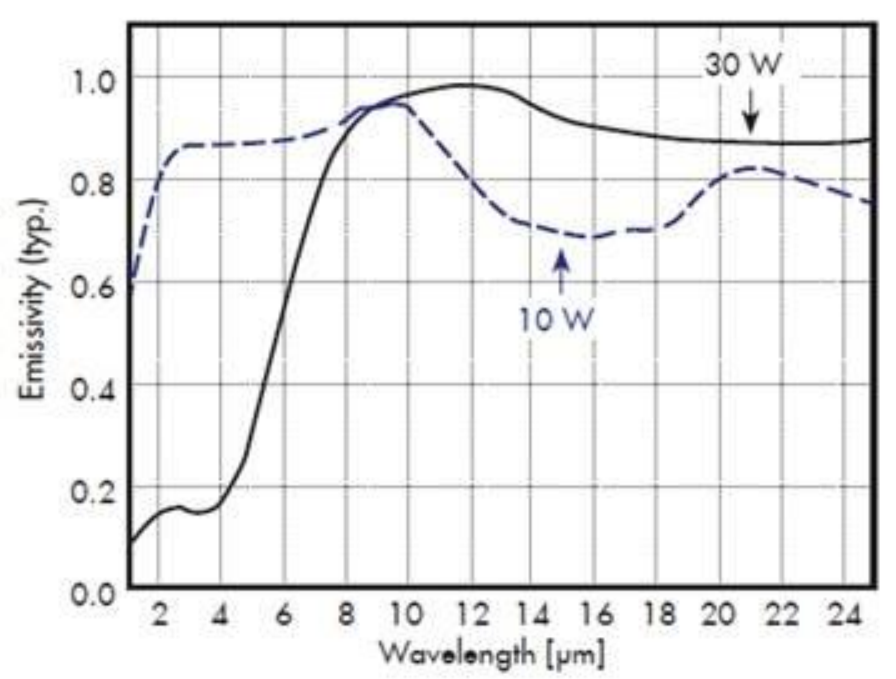

Fig. 3.1: Wavelength dependence of emissivity for the IR light source. The figure taken from [104].

The IR lamp emits light in all directions which means it has lower light intensity in the required direction. Hence, the IR lamp is combined with a reflector mirror to increase the light intensity and to control the light direction. The mirror is placed close to the IR lamp in an appropriate height and angle that allows the maximum light intensity to be coupled into the monochromator (Horiba Scientific, iHR320) as shown in Fig. 3.2. The main use of the monochromator is to select the wavelength precisely to measure $E_{\mathrm{g}}$ (see Fig. 3.3). The monochromator has high accuracy of $\pm 0.20 \mathrm{~nm}$ in the wavelength and a step size of less than $1 \mathrm{~nm}$. It has two diffraction gratings (Horiba Scientific, 51031 and 51038) that cover the wavelength range between 3 and $20 \mu \mathrm{m}$ with relative efficiency between $\sim 40$ and $80 \%$ (see Fig. 3.4). The gratings were switched at the wavelength of $9.5 \mu \mathrm{m}$. The wavelength was selected through the LabView program developed (see Appendix 1). 


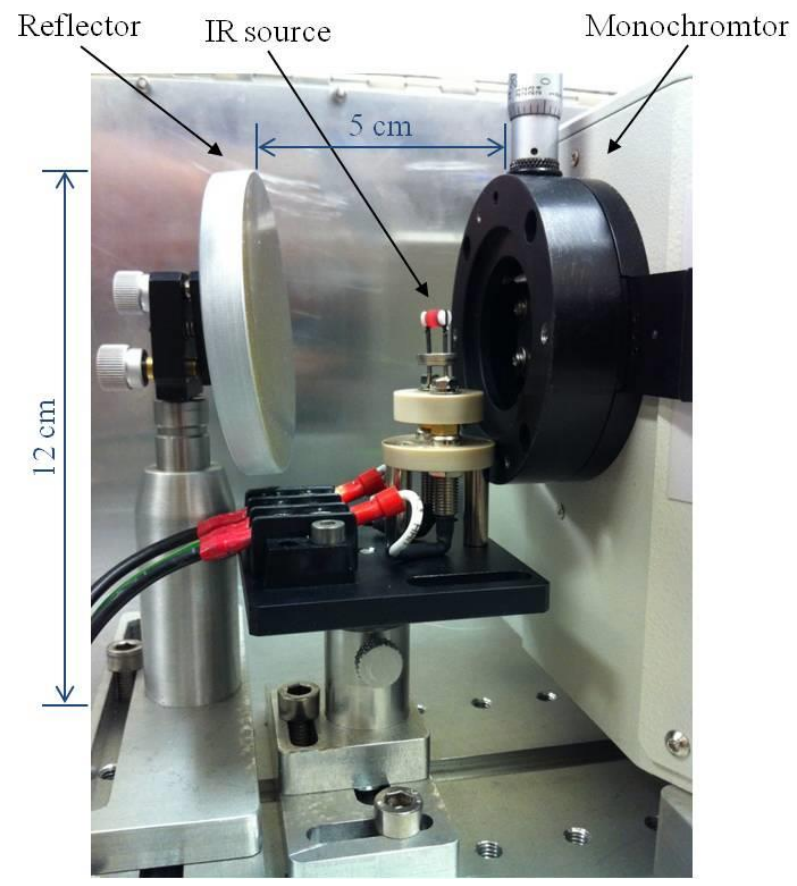

Fig. 3.2: Configuration of the IR lamp, the reflector mirror and the monochromator.

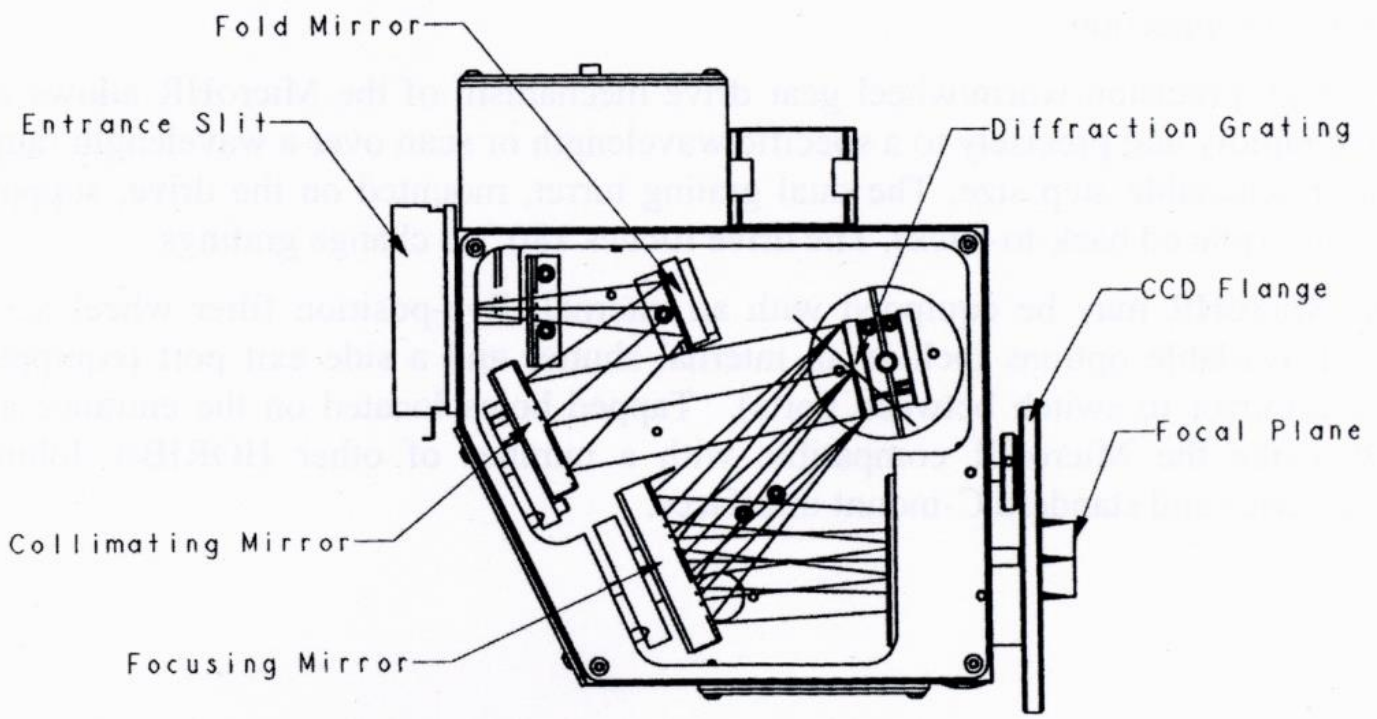

Fig. 3.3: Schematic diagram of the monochromator. The figure taken from [105]. 


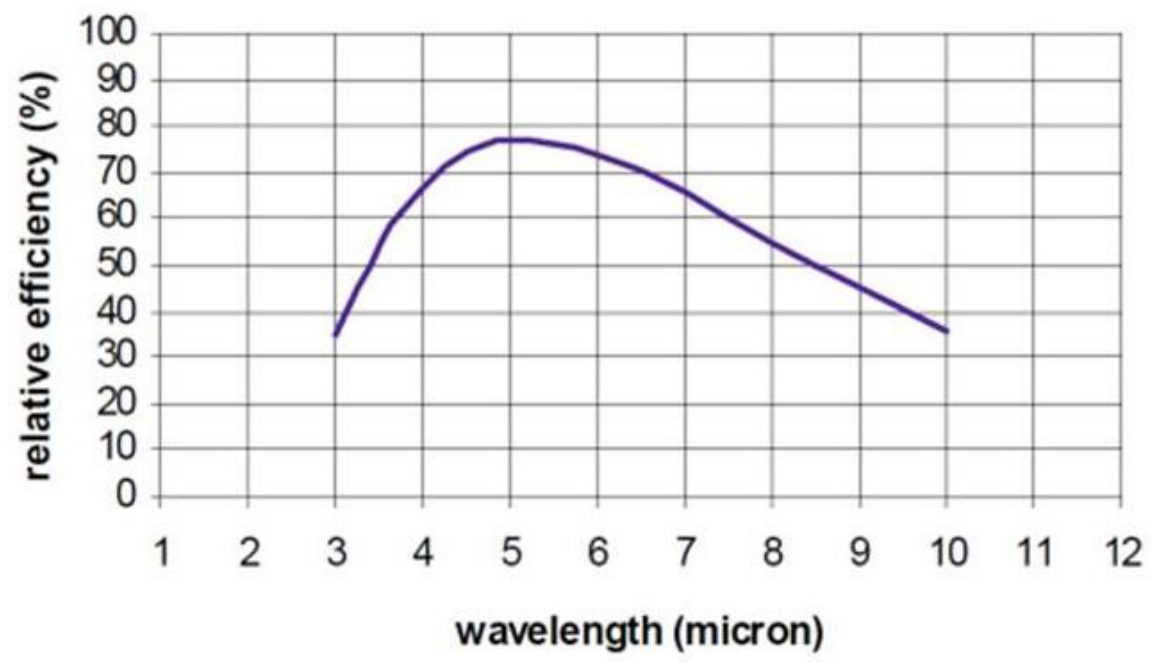

$51038--$

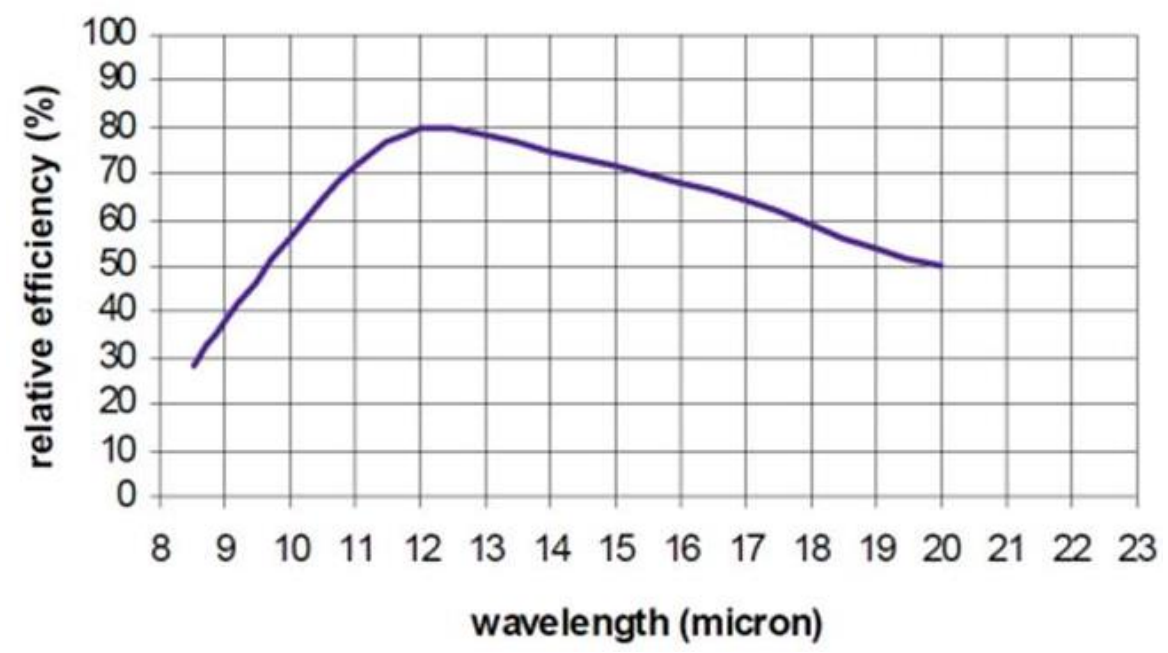

Fig. 3.4: Efficiency distribution for the two diffraction gratings. The figure taken from [106].

\subsubsection{Pulse generation}

The second criterion is to have a pulse light to excite the minority spin electrons in the sample for a lock-in measurement. This is required to convert the continuous IR light into a pulse. A simple way to do this is to use an optical chopper. In this study an optical chopper (Signal Recovery, model 197) was used. It has a frequency $(f)$ range between 15 and $3000 \mathrm{~Hz}$. The different frequencies were examined to optimise the frequency for this measurement. The higher frequencies $(>1.5 \mathrm{kHz})$ offered a well-defined square 
pulse shape, which was ideal for locking with the received signals from a photodetector [see Fig. 3.5(a)]. However, the higher frequencies had poor signal to noise ratios ( $26-$ 20) because of the high noise level $(\sim 20-30 \mathrm{mV})$ according to the following formula:

$$
\operatorname{SNR}\left(\frac{\mathrm{S}}{\mathrm{N}}\right)=20 \log _{10} \frac{V_{S}}{V_{n}}
$$

where $V_{\mathrm{s}}$ is main signal strength and $V_{\mathrm{n}}$ is the noise signal strength.

The lower frequencies $(<100 \mathrm{~Hz})$ had better signal to noise ratios. In this study, all the measurements were carried out at the frequency of $17 \mathrm{~Hz}$. This frequency also generated with the optimum stability $( \pm 0.1 \mathrm{~Hz})$. The output from the chopper was used as a reference signal to be fed to a lock-in amplifier.
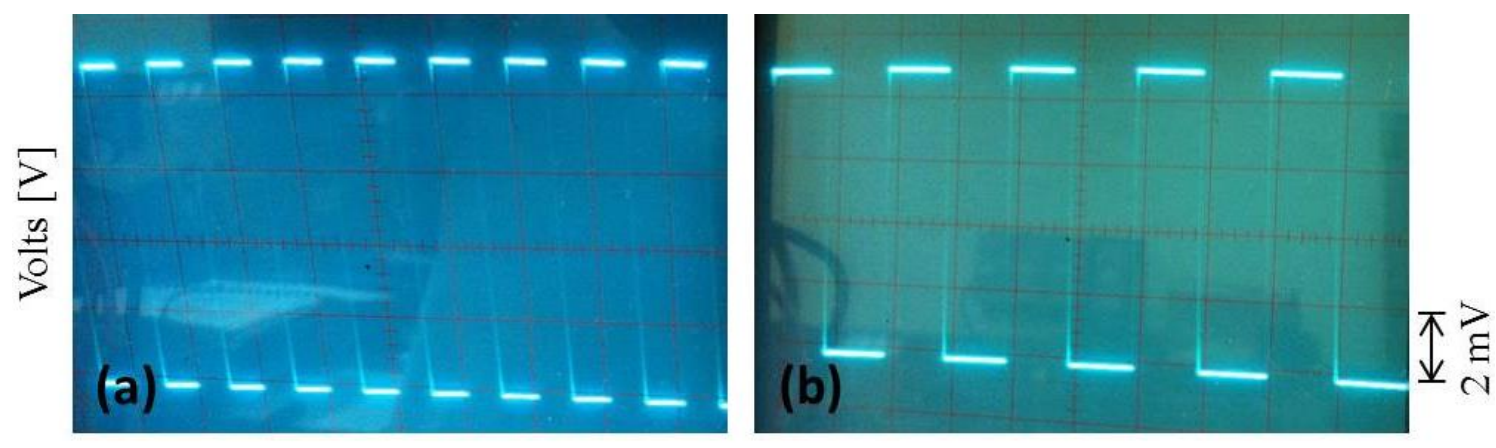

Time $\lceil\mathrm{S}\rceil$

Fig. 3.5: Signals of the optical chopper operated at $f=$ (a) $1 \mathrm{kHz}$ and (b) $17 \mathrm{~Hz}$.

\subsubsection{Circular polarisation}

The third criterion is to generate circularly-polarised light which allows me to generate photoexcited spin-polarised electrons with energy corresponding to the band-gap of the Heusler alloy films (i.e., $\sim 0.062-0.62 \mathrm{eV})$. In order to achieve the photoexcitation, a linear polariser (Edmund Optics, model KRS-5 62774) was first used to polarise the IR light linearly based on its efficient transmission over $70 \%$ in a wide range $3 \leq \lambda \leq 20 \mu \mathrm{m}$ (see Figs. 3.6 and 3.7). The linear polarisation was achieved at an angle of $45^{\circ}$ tilt from the vertical optical axis as shown in Fig. 3.8. The linearly polarised IR light was then 
introduced to a $\lambda / 4$-wave plate (II-VI, FRZ-2-14-0.75-90-RM) to convert it to circular polarised light, covering the wavelength range between 2 and $14 \mu \mathrm{m}$ (see Fig. 3.9).

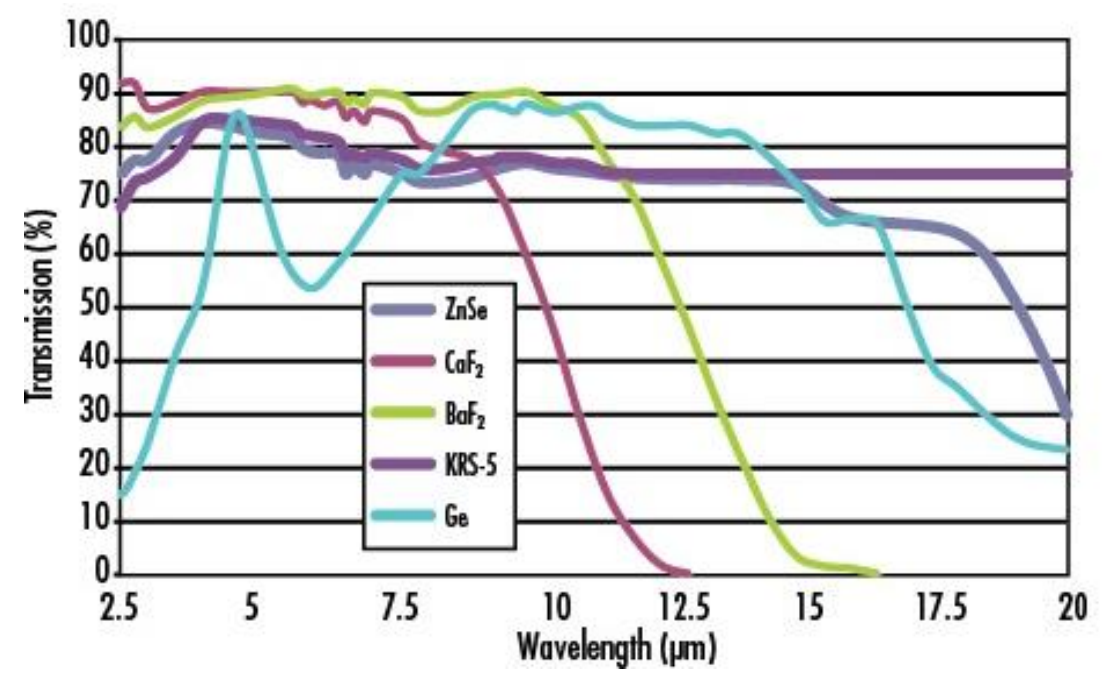

Fig. 3.6: Typical maximum transmission for linearly polarised light for different types of polarisers. The figure taken from [107].

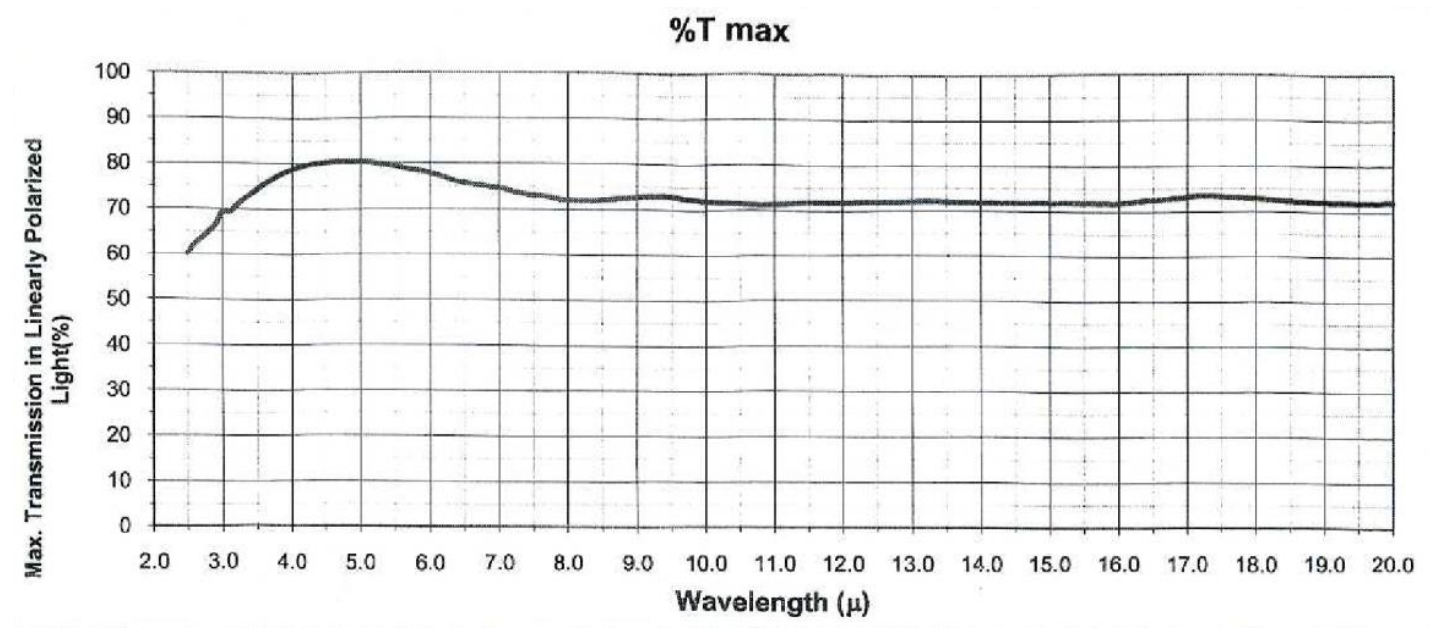

Fig. 3.7: Maximum transmission of the KRS-5 linear polariser. The figure taken from [108]. 


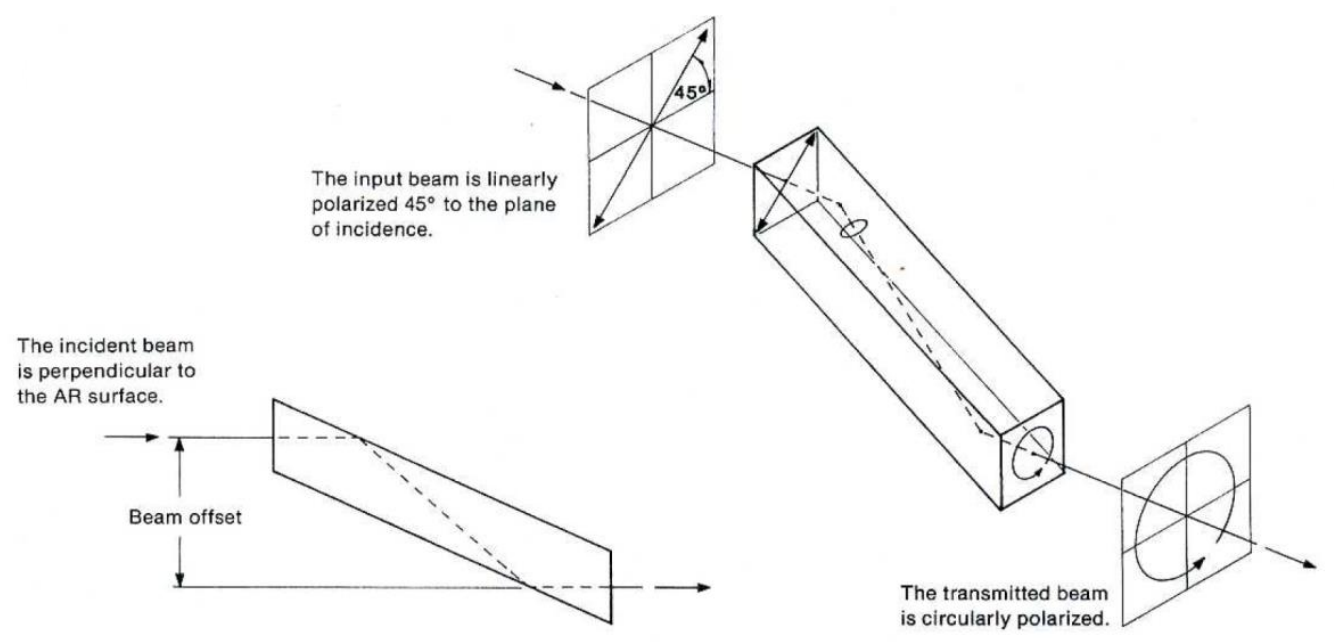

Fig. 3.8: Schematic diagram of the $\lambda / 4$-wave plate. The figure taken from [109].

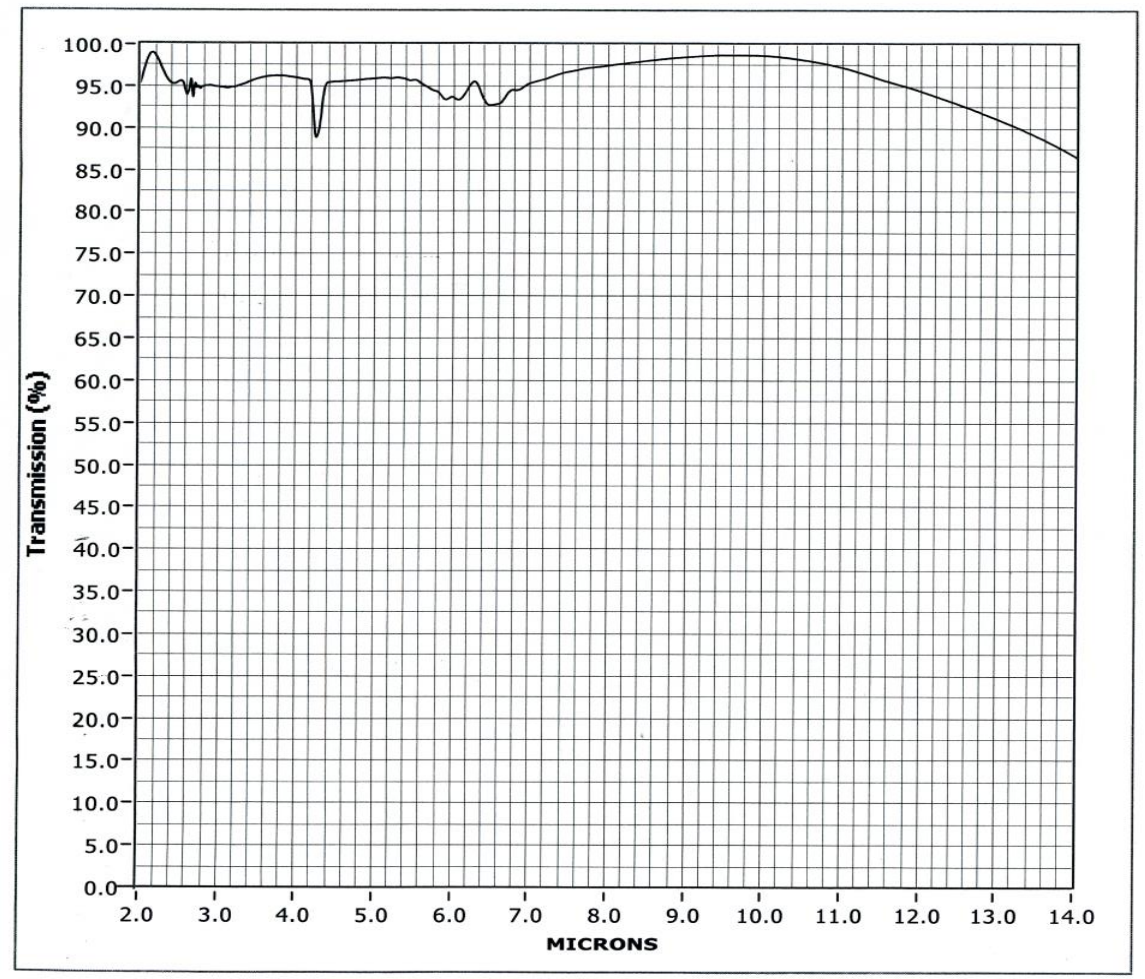

Fig. 3.9: Wavelength dependence of the transmission efficiency for the $\lambda / 4$-wave plate. The figure taken from [110].

\subsubsection{Photodetector}

A photodetector needs to work in the wavelength range corresponding to the band-gap energy, and has to detect low signal intensities (typically $\sim 1 \mathrm{mV}$ ). Different types of 
photodetectors have been investigated such as mercury cadmium telluride (MCT or HgCdTe) detectors, thermistors, thermopiles, photodiodes and pyroelectrics (see Table 3.2 for comparison of these types). Each type had advantages and disadvantages in terms of the optical bandwidth, transmittance, responsivity (i.e., sensitivity), electronic circuitry, noise, operational temperature, element size and cost. The MCT was the first choice due to its capability to fulfil our requirements as follows: It works at the required wavelength range (i.e., $\sim 2<\lambda<25 \mu \mathrm{m}$ ) with very high detectivity $\left(D^{*}\right)$ of $\sim 5 \times 10^{9}$ $\mathrm{cm} \cdot \mathrm{Hz}^{1 / 2} / \mathrm{W}$ and low noise of $\sim 20 \mathrm{nV} / \mathrm{Hz}^{1 / 2}$. It is also provided with the associated electronic circuit for amplification. However, it has two main drawbacks that led to be excluded in this study. Firstly, it works at low temperature (typically at 77K), requiring cooling the detector with liquid $\mathrm{N}_{2}$. Secondly, it is very expensive (typically $£ 6,125$ ). Thermopiles and thermistors are then considered to be good choices due to their spectral range, high detectivity $\left(D^{*}\right) \sim 3 \times 10^{8} \mathrm{~cm} \cdot \mathrm{Hz}^{1 / 2} / \mathrm{W}$, low noise of $\sim 12 \mathrm{nV} / \mathrm{Hz}^{1 / 2}$, provision of a preamplifier and low cost (typically £142). However, it has a major drawback of working only with continuous light, whereas my design requires a pulsed light. Hence, the thermopiles and thermistors have been excluded as well. A photodiode is also a choice to be used. Its main advantages are $D^{*}$ of $\sim 5 \times 10^{8} \mathrm{~cm} \cdot \mathrm{Hz}^{1 / 2} / \mathrm{W}$ and low cost. However, the photodiode does not have wide spectra, so several photodiodes were needed to cover the entire wavelength spectra in this study, which is not practical. Also, some photodiodes require a complicated electronic circuit to amplify the received signals. 
Table 3.2: Comparison of different types of detectors.

\begin{tabular}{|c|c|c|c|c|}
\hline \multirow[b]{2}{*}{ Specifications } & \multicolumn{4}{|c|}{ Detector type } \\
\hline & $\begin{array}{l}\text { Mercury } \\
\text { Cadmium } \\
\text { Telluride } \\
\text { (MCT) }\end{array}$ & $\begin{array}{l}\text { Thermopiles \& } \\
\text { thermistors }\end{array}$ & Photodiodes & Pyroelectrics \\
\hline $\begin{array}{c}\text { Optical } \\
\text { bandwidth } \\
(\mu \mathrm{m})\end{array}$ & $2-25$ & $2-30$ & $2.5-4.8$ & $\sim 6-16$ \\
\hline $\begin{array}{l}\text { Active area } \\
\text { (mm) }\end{array}$ & $1.0 \times 1.0$ & $2.0 \times 2.0$ & 0.3 dia. & $2.5 \times 2.5$ \\
\hline $\begin{array}{l}\text { Responsivity } \\
\text { (V/W) }\end{array}$ & 250 & $\begin{array}{c}18.9 \\
\text { (typical) }\end{array}$ & $\begin{array}{c}\text { (Sensitivity) } \\
0.6-0.8 \mathrm{~A} / \mathrm{W}\end{array}$ & $87.8 \times 10^{3}$ \\
\hline $\begin{array}{c}\text { Detectivity D* } \\
(\mathbf{c m} \sqrt{ } \mathbf{H z} / \mathbf{W})\end{array}$ & $5 \times 10^{9}($ typical $)$ & $3 \times 10^{8}$ & {$[5-8] \times 10^{8}$} & $\sim 0.8 \times 10^{9}$ \\
\hline $\begin{array}{c}\text { Noise } \\
\text { equivalent- } \\
\text { power }(\mathbf{N E P}) \\
(\mathbf{W} / \sqrt{ } \mathbf{H z})\end{array}$ & $1 \times 10^{-11}$ & $0.68 \times 10^{-9}$ & - & $3.29 \times 10^{-9}$ \\
\hline Detector type & KRS-5 & - & - & $\mathrm{LiTaO}_{3}$ \\
\hline Noise & $\sim 20 \mathrm{nV} / \sqrt{\mathrm{Hz}}$ & $12 \mathrm{nV} / \sqrt{\mathrm{Hz}}$ & - & $291 \mu \mathrm{Vrms} / \sqrt{\mathrm{Hz}}$ \\
\hline $\begin{array}{c}\text { Operating } \\
\text { temperature } \\
\text { ( C) }\end{array}$ & -196 & -40 to +85 & -15 to +40 & -40 to +70 \\
\hline $\begin{array}{c}\text { Electronic } \\
\text { circuitry }\end{array}$ & Provided & Provided & Not provided & Provided \\
\hline Cost $(\mathfrak{E})$ & 6,125 & $\sim 142$ & $\sim 210$ & 120 \\
\hline Ref. & 111 & 112 & 113 & 114 \\
\hline
\end{tabular}

In this study, the choice was therefore made to use the pyroelectric as it fulfilled all of the requirements as discussed above: It has high $D^{*}$ of $\sim 1.13 \times 10^{8} \mathrm{~cm} \cdot \mathrm{Hz}^{1 / 2} / \mathrm{W}$, low noise of $\sim 291 \mu \mathrm{V} / \mathrm{Hz}^{1 / 2}$, provision of a preamplifier and low cost. The pyroelectric is a thermal sensor that converts the incoming light into an electric signal to be measured. A pyroelectric detector (ELTEC, Pyroelectric IR detector model 441) has been used to detect the IR signal reflected from the sample surface. The pyroelectric was a single-element lithium tantalite $\left(\mathrm{LiTaO}_{3}\right)$ and had several additional advantages such as relatively wide spectral range $(\sim 6<\lambda<16 \mu \mathrm{m})$, high transmittance average above $75 \%$ in the wavelength range between 6.7 and $14 \mu \mathrm{m}$ (see Fig. 3.10), high 
sensitivity of $\sim 88 \mathrm{kV} / \mathrm{W}$, ambient operation temperature $\left(-40^{\circ}\right.$ to $\left.+70^{\circ} \mathrm{C}\right)$, large active area of $2.5 \times 2.5 \mathrm{~mm}$ and reasonable cost $(£ 150)$.

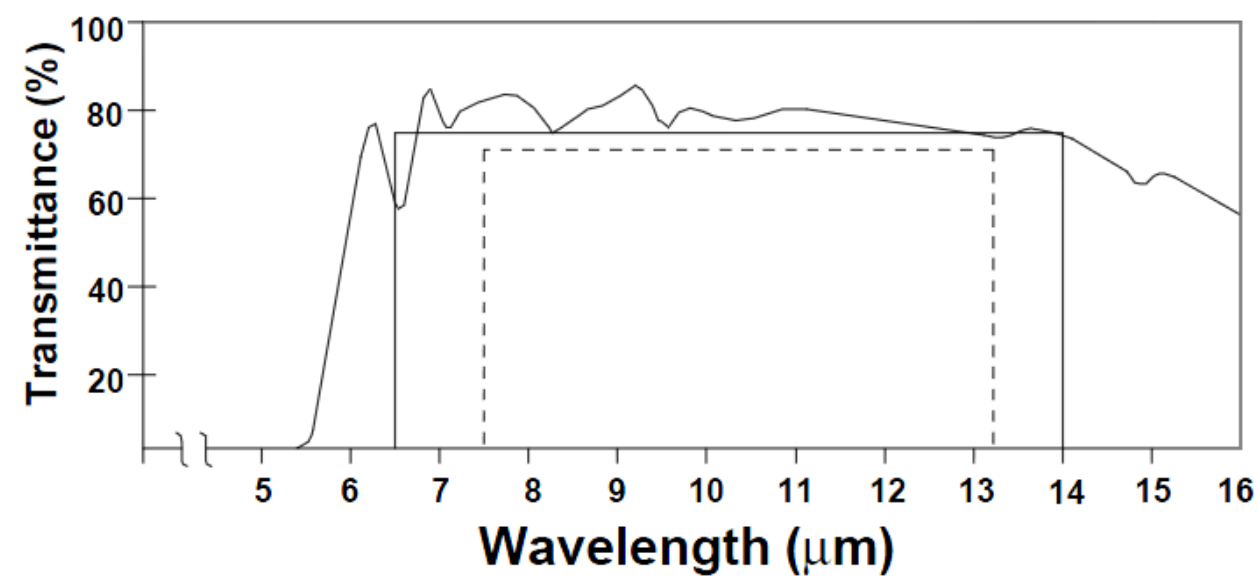

75\% Transmission Average

$70 \%$ Transmission Absolute

Fig. 3.10: Transmission characteristics of the IR photodetector used in this study. The figure taken from [114].

FIELD OF VIEW

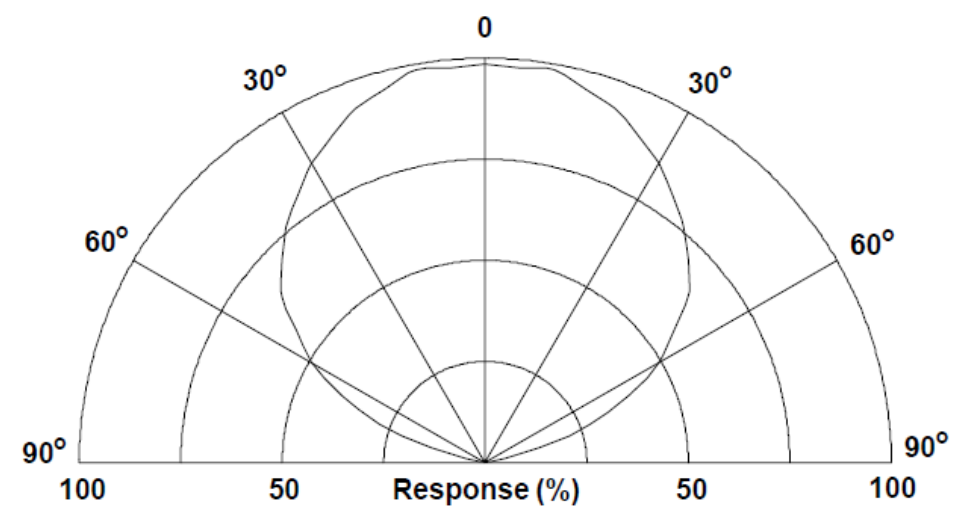

Fig. 3.11: Response of the photodetector at different view angles. The figure taken from [114]. 


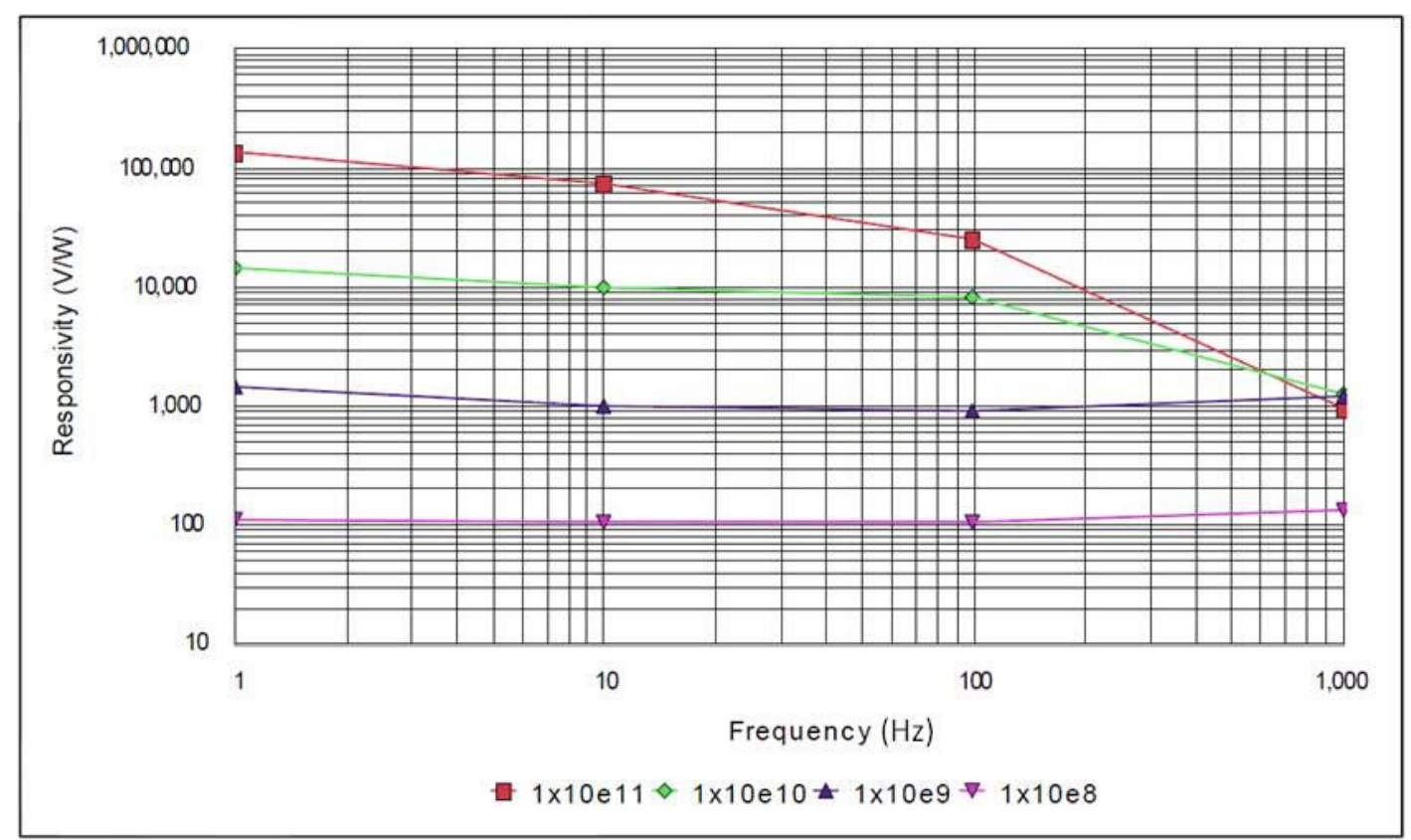

Fig. 3.12: Pyroelectric phototdetector (Model 441) with various feedback resistor values. The figure taken from [115]. In this study, a feedback resistor of $1 \times 10^{11} \Omega$ was used.

\subsubsection{Optical matching}

Matching in the wavelength range needs to be achieved optically among all the components used in order to ensure the highest measurement efficiency. The IR light source has been selected initially based on its wavelength spectra, corresponding to the band-gap energy to be measured, in addition to other specifications as mentioned in the previous subsections. Other optical components were then selected to be operated in this spectrum range (or most of it). Two diffraction gratings for the monochromator were selected to complement the entire wavelength range. Linear and circular polarisers were also selected to maximise their efficiency in this wavelength range with additional consideration on their diameters. The photodetector was also specified to maximise its sensitivity in this wavelength. 


\subsubsection{Lock-in technique}

The sixth criterion is to be able to lock-in the received signals from the photodetector against a reference signal from the output of the optical chopper. This has been achieved using a lock-in amplifier (Signal Recovery, model 7265). The reflected light from the film was used as a main signal and the chopper signal used as a references with around $0^{\circ}$ phase shift between the two signals. Auto-measure function of the lock-in amplifier was not sensitive enough to measure the signals initially due to the noise. Therefore, some parameters in the lock-in amplifier had to be optimised to minimise the noise. In this study, time constant, sensitivity and settling time were selected to be $200 \mathrm{~s}, 20 \mathrm{mV}$ and 200 s, respectively.

\subsubsection{Sample holder}

The seventh criterion is to make a simple design of the sample holder which holds the sample with and without a permanent magnet. The sample holder has been designed to have two adjacent grooves, one for the magnet and another for the sample. This allowed me to hold either only the sample or both the sample and the magnet in the same position. The magnet was used to apply a magnetic field perpendicular to the sample. It could adjust the sample position with an accuracy of 240 arcsecond to introduce the IR circularly-polarised light beam spot onto the centre of the sample. In addition, the height of the sample could be adjusted through the post. The incidence angle of the IR light was set to be at $45^{\circ}$ from the film normal and the reflected intensity was measured by the photodetector. The incidence angle (i.e., $45^{\circ}$ ) was selected to give the largest signals from the photodetector. The permanent magnet used had larger diameter ( $11 \mathrm{~mm})$ than the films $(5 \mathrm{~mm} \times 5 \mathrm{~mm})$ to ensure that the magnetic field passing through the film 
was exactly perpendicular and not to be diverged at the edges of the magnet or the film. In order to ensure this, the film was positioned in the centre of the magnet surface.

\subsubsection{Optical paths}

The final criterion is to maintain the minimum light travelling distance in order to keep the signal intensities as large as possible. This is because the IR intensity is reduced almost exponentially with respect to the travelling distance [116][117]. These optical paths have been optimised once all the above criteria were met. This also included aligning the components as discussed in the following Section 3.2. before running the measurements. This eventually provided typical intensity of $\sim 25 \mathrm{mV}$, requiring further reduction of the noise level as discussed in Section 3.4.

\subsection{Alignment}

As discussed in the previous Section 3.1., the experimental set-up consists of the following components as shown in Fig. 3.13; the IR light source, the monochromator, the optical chopper, the linear polariser, the $\lambda / 4$-wave plate, the sample holder, the photodetector with an internal preamplifier, the lock-in amplifier and computer to control the measurements via the LabView programme. The alignment has been carried out in several steps to ensure the maximum beam intensity to pass through all the components. Hence, it is discussed separately in the following subsections. 


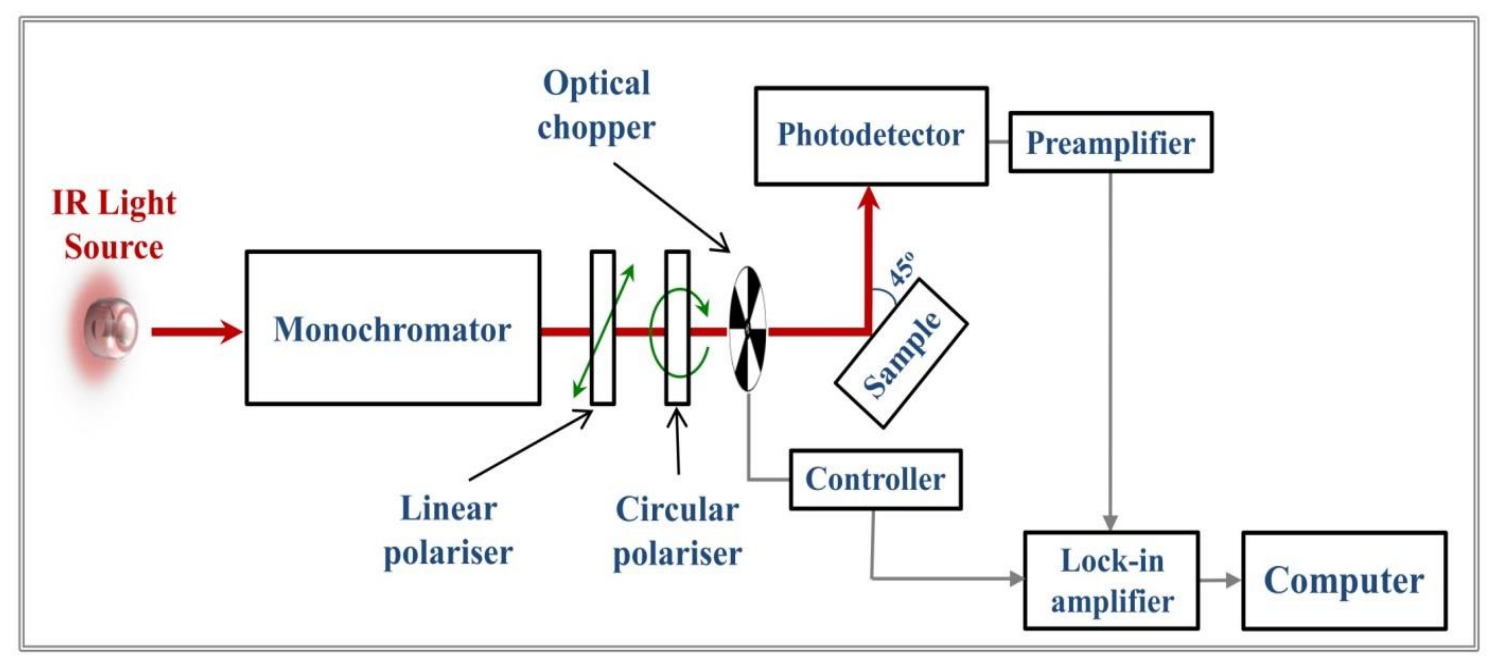

Fig. 3.13: Schematic diagram of the experimental set-up.

\subsubsection{IR light source and monochromator}

The IR light source was held on a post and post holder, so that its position can be adjusted precisely $( \pm 12 \mathrm{~mm})$. Then the IR source was coupled to the monochromator by placing it very close $(\sim 16 \mathrm{~mm})$ to the incident slit of the monochromator. A concave mirror was then placed on an adjustable holder on the other side of the IR source to maximise the intensity of the IR light. The IR light has to pass through the centre of the incident slit of the monochromator. For this adjustment, an IR card was needed as a simple way to see the IR light from the card reflection. However, it was not possible to find an IR card in the mid-IR range at a reasonable cost. Therefore, the pyroelectric photodetector was used to detect the IR light and to measure the intensity distribution of the IR light source. Initially, the intensity of the IR light was measured to confirm its distribution over the wavelength range. The pyroelectric photodetector was placed close to the centre of the divergence slit of the monochromator perpendicularly to the photodetector surface. The IR light source was turned on and left for about thirty minutes to stabilise its radiation. The monochromator was set at a specific wavelength (i.e., $3 \mu \mathrm{m}$ ) to begin with. The photodetector was used to measure the output intensity to compare it with the corresponding intensity provided by the IR source supplier (see Fig. 
3.14, dashed line). This process was repeated at different wavelengths by tuning the grating at $20 \mathrm{~nm}$ steps (see Fig. 3.15).

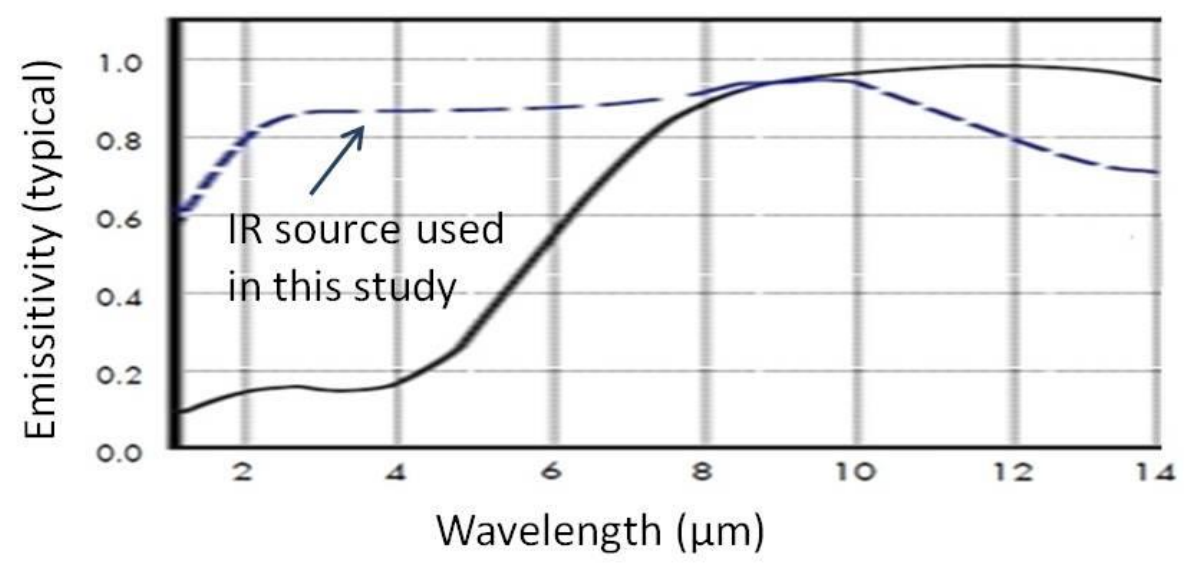

Fig. 3.14: Reference intensity distribution of the IR light source. The figure taken from [104].

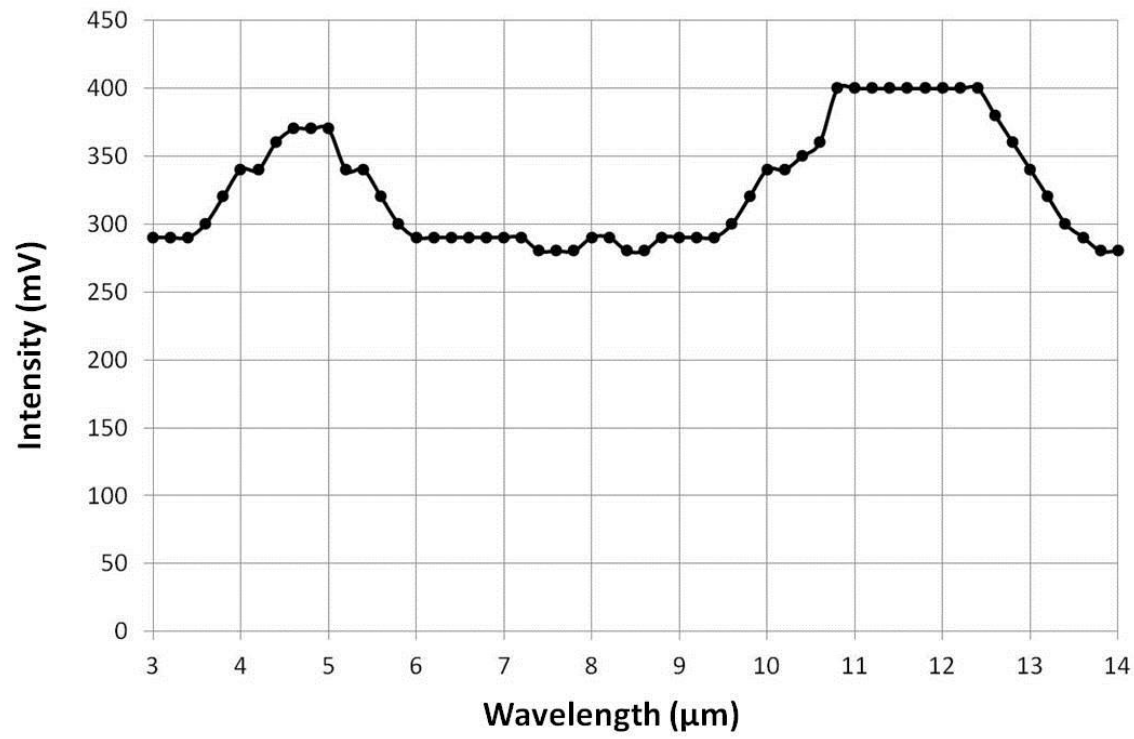

Fig. 3.15: Measured intensity distribution of the IR light source at $20 \mathrm{~nm}$ steps.

The measured IR light distribution matched qualitatively with the catalogue provided by the supplier (see Figs. 3.14 and 3.15). However, the differences in the IR light distribution between them were due to the differences in measurement environment. Some companies have measured their IR properties in a chamber with 
flowing argon gas to reduce the chance of IR photon absorption by $\mathrm{H}_{2} \mathrm{O}$, especially in the wavelength range between 5.5 and $8 \mu \mathrm{m}$ (see Fig. 3.16).

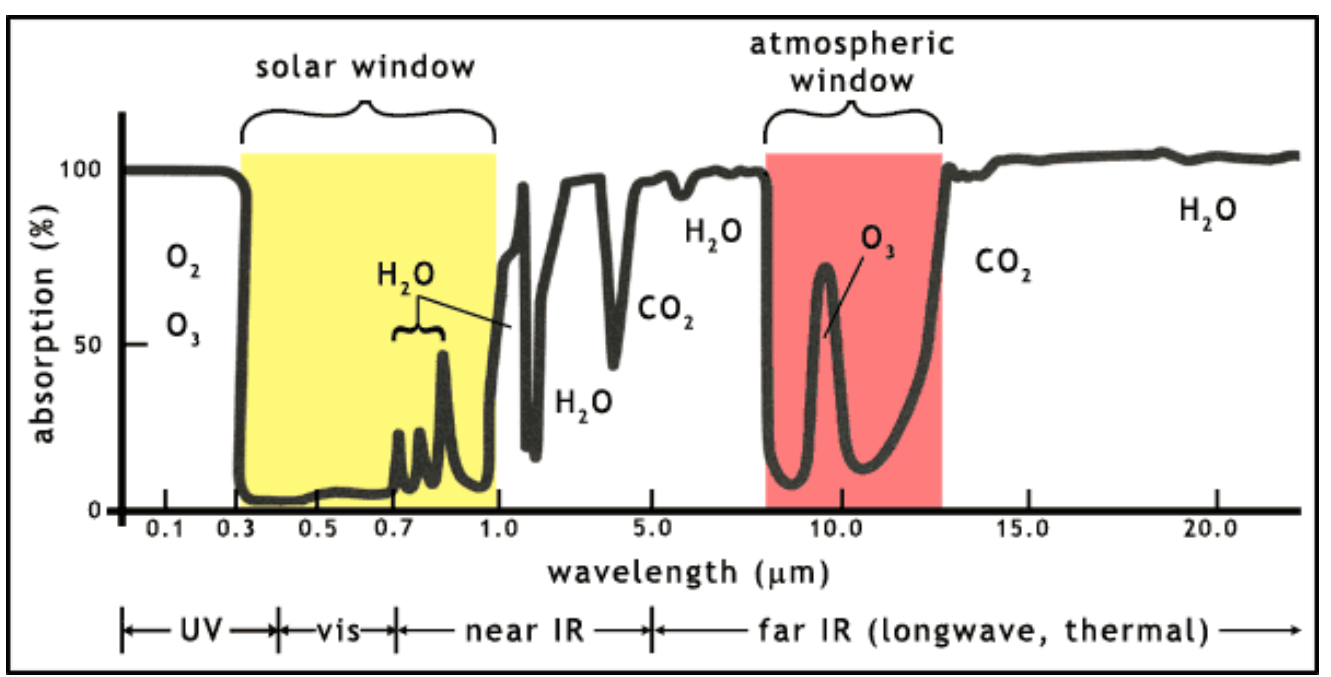

Fig. 3.16: Reference intensity distribution of the IR light source. High target utilisation sputtering. The figure taken from [118].

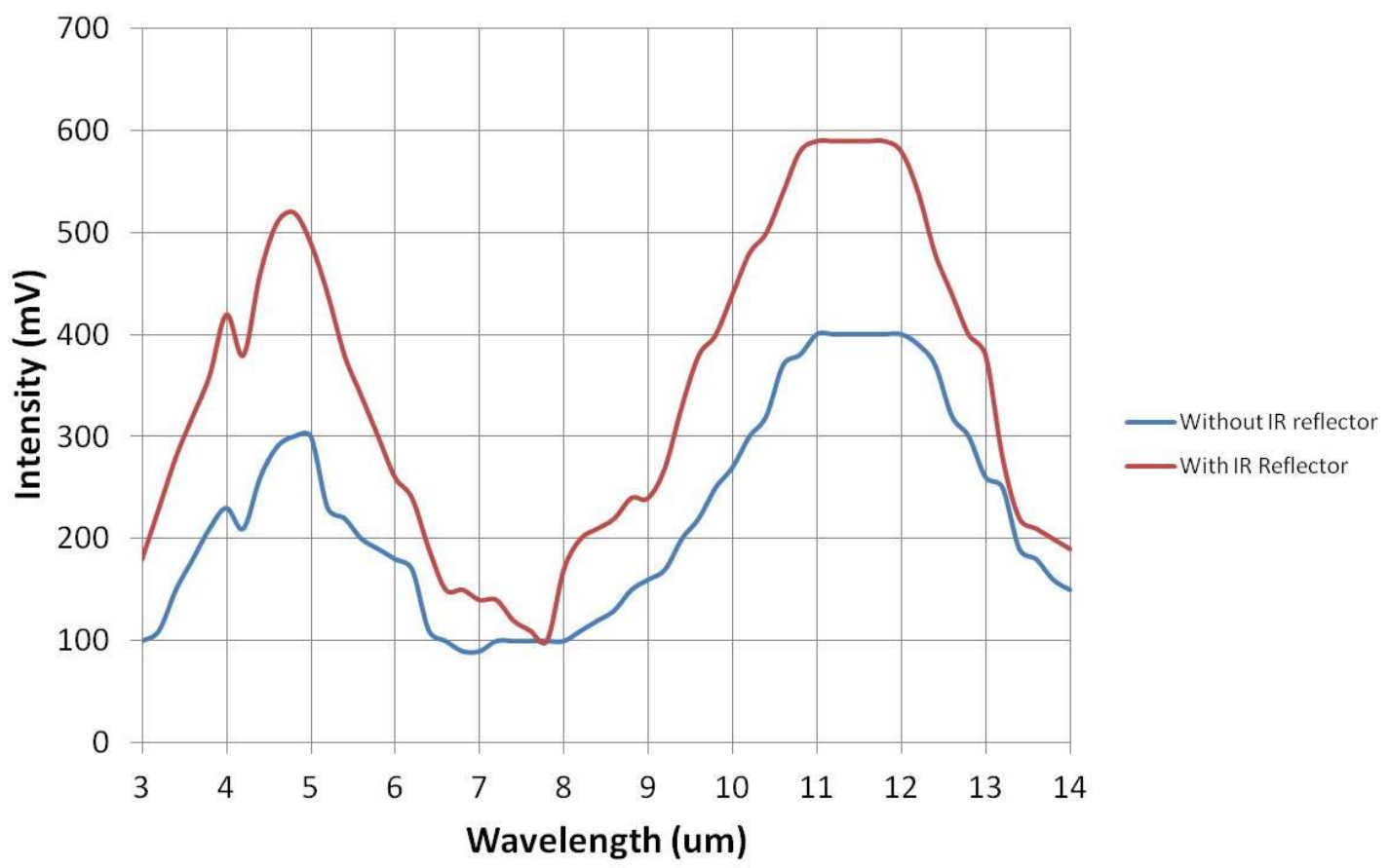

Fig. 3.17: The measured IR light intensity with (red line) and without (blue line) reflector.

In order to maximise the intensity, the IR source was moved by $\pm 15 \mathrm{~mm}$ in three axes around the centre of the incident slit while monitoring the intensity through the 
photodetector until receiving the highest signal. The highly sensitive photodetector was very beneficial for this task. The position of the IR source was then fixed. The next step was adjusting the concave mirror to maximise the light coupling into the monochromator. The concave mirror could increase the intensity by up to $\sim 67 \%$ as can be seen in Fig. 3.17. Once the largest signal was obtained, the concave mirror and the monochromator were fixed at their positions.

\subsubsection{Optical chopper}

The optical chopper was positioned in front of the monochromator in a way that the aperture of the optical chopper was close to the centre of the divergence slit of the monochromator. This allowed the maximum intensity of the IR light to pass through the chopper and to reach the following components.

\subsubsection{Linear and circular polarisers}

The linear polariser was placed in front of the optical chopper to be perpendicularly to the optical path. The centre of the linear polariser was aligned to be at the centre of the aperture of the optical chopper to introduce the maximum and symmetric beam. In order to generate circularly-polarised light, the $\lambda / 4$-wave plate was placed in parallel with the linear polariser where the centre of the linear polariser was facing the centre of the $\lambda / 4$ wave plate. After the $\lambda / 4$-wave plate, the intensity was measured to be in the range between $\sim 380$ and $590 \mathrm{mV}$ for $\sim 10<\lambda<13 \mu \mathrm{m}$ and it dropped by $\sim 87-90 \%(\sim 40-$ $75 \mathrm{mV}$ ). It is expected for the intensity to drop down as half of the beam was eliminated by the linear polariser and due to the optics absorption of the light. 


\subsubsection{Photodetector}

The pyroelectric photodetector with its associated electronic circuit was enclosed in an aluminium box and placed close $(\sim 22 \mathrm{~mm})$ to the film and it was fixed at $45^{\circ}$ from the film normal to maximise the reflection intensity (see Fig. 3.13). This angle is sensitive to both the in-plane and plane-normal components of the spin polarised electrons as the $45^{\circ}$ axis can be defined as a sum of these components.

\subsubsection{Sample holder}

The sample holder was mounted on a post and both of them were placed on a rotary stage in order to control their position and angle. In this study, the angle was fixed at $45^{\circ}$ as a maximum light reflection occurs at this angle.

\subsection{Band-gap measurements}

After completing the optical alignment, the band-gap measurements were performed. The monochromator was initialised by opening the entrance and exit slits to their full width (i.e., $2 \mathrm{~mm}$ ) and the wavelength was set to $3 \mu \mathrm{m}$, which tuned then until $16 \mu \mathrm{m}$. The frequency of the optical chopper was set to $17 \mathrm{~Hz}$. A sample (i.e., $\mathrm{Co}_{2} \mathrm{FeSi}$, $\mathrm{Co}_{2} \mathrm{FeAl}_{0.5} \mathrm{Si}_{0.5}$ and $\mathrm{NiMnSb}$ ) was then placed on the sample holder at the right position (i.e., at $45^{\circ}$ from the light entrance and at $45^{\circ}$ to the photodetector). Last step was to set the parameters of the lock-in amplifier as follow: time constant: 200 s, sensitivity: 20 $\mathrm{mV}$, settling time: $200 \mathrm{~s}$ and phase shift: $\sim 0^{\circ}$. Here, the time constant should be long enough to minimise the output noise. In this study, the set value was found to reduce the noise level to be below $20 \mathrm{mV}$. The sensitivity was another parameter to be optimised at $20 \mathrm{mV}$. This value was set according to the changes in the signal level and to provide the most accurate reading of the signal value with $1 \mu \mathrm{V}$ resolution. The settling time is the time required between every two consecutive signal inputs. The optimum value was 
adequate for preventing the influence of quick changes in the signal values as the signal input needs $\sim 500 \mathrm{~ms}$.

A typical band-gap measurement was carried out by conducting a set of three separate measurements for the same film. The first measurement was made without applying a magnetic field where the intensity of the reflected light from the sample surface was measured over the entire wavelength range. The second measurement was made with applying a perpendicular magnetic field by attaching a magnet on the back of the film with the S-pole upwards. The magnet has sufficient field strength (i.e., $\sim 4 \mathrm{kOe}$ ) to magnetise the film. The third measurement was similar to the second one except the orientation of the magnetic field which was reversed (N-pole upwards). The band-gap can be determined by subtracting the N-pole-up from the S-pole-up results. The details will be discussed in the following three chapters.

\subsection{Measurements improvement}

Initial band-gap measurements had relatively high noise levels $(\sim 30 \mathrm{mV})$ due to many reasons such as the photodetector internal noise, surrounding light, body heat radiation and electromagnetic radiation from the high power cables close to the experimental setup. This noise level was determined when the lock-in parameters was set as follows: time constant: $100 \mathrm{~s}$, sensitivity: $50 \mathrm{mV}$, settling time: $50 \mathrm{~s}$ and phase shift: $\sim 0^{\circ}$. Therefore, reducing the noise to signal ratio was an important step to make the measurements with less noise by trying to isolate the experimental set-up as possible from the surrounding which led to design a shielding box. Additionally, modifying the electronic circuit of the photodetector was another step was taken. More details were discussed below. 


\subsubsection{Electronic circuit}

The photodetector could not measure efficiently low signals if the noise level exceeds $20 \mathrm{mV}$. So, an additional electronic circuit was designed to reduce the noise level (see Appendix 2). It contains a series of resistors and different types of capacitors with different capacitance values. By adding this circuitry to the photodetector, the noise level reduced to $\sim 20 \mathrm{mV}$ or less. The photodetector and its associated electronic circuit were enclosed in a small aluminium box for better protection from the electromagnetic noise and for easy handling (see Fig. 3.18).

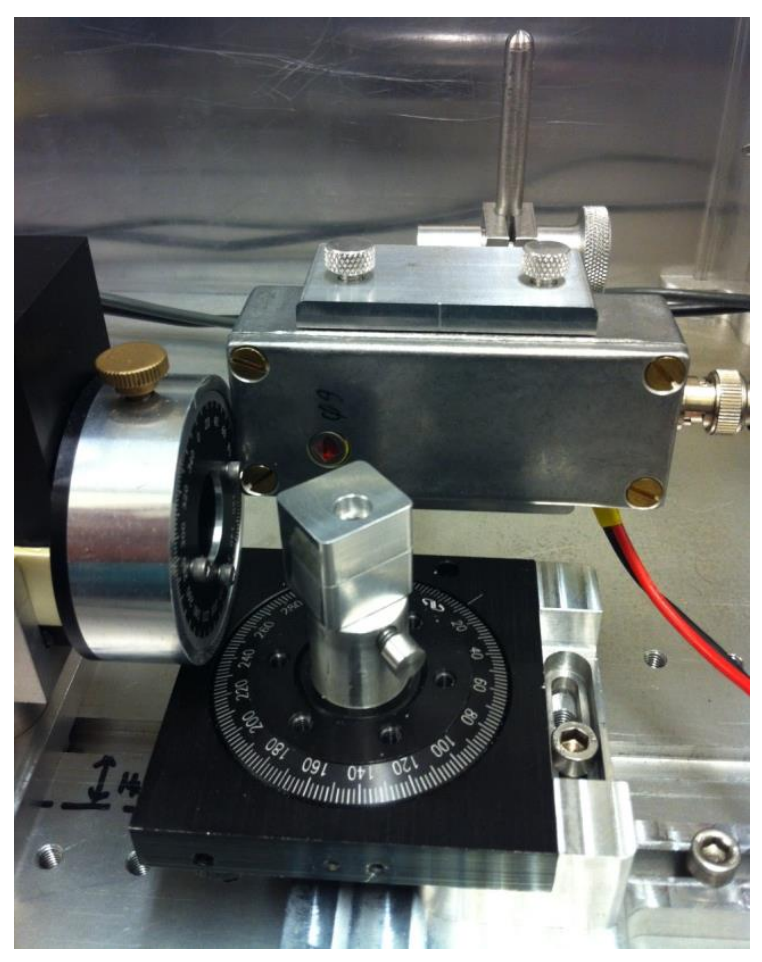

Fig. 3.18: Photodetector in the aluminium box.

\subsubsection{Shielding box}

For further reduction of the noise level, a shielding box was fabricated. All the optical components were enclosed inside this box. Both the lock-in amplifier and the power supply of the IR light source stayed outside the box. The box and screws were made from non-magnetic materials to prevent any magnetic field response. It contains base 
with holes and grooves to allow moving each component individually and fix it at the right position (see Fig. 3.19). Also, it has some small holes (diameters of $10-19 \mathrm{~mm}$ ) at sides to take out power cables and other wires.

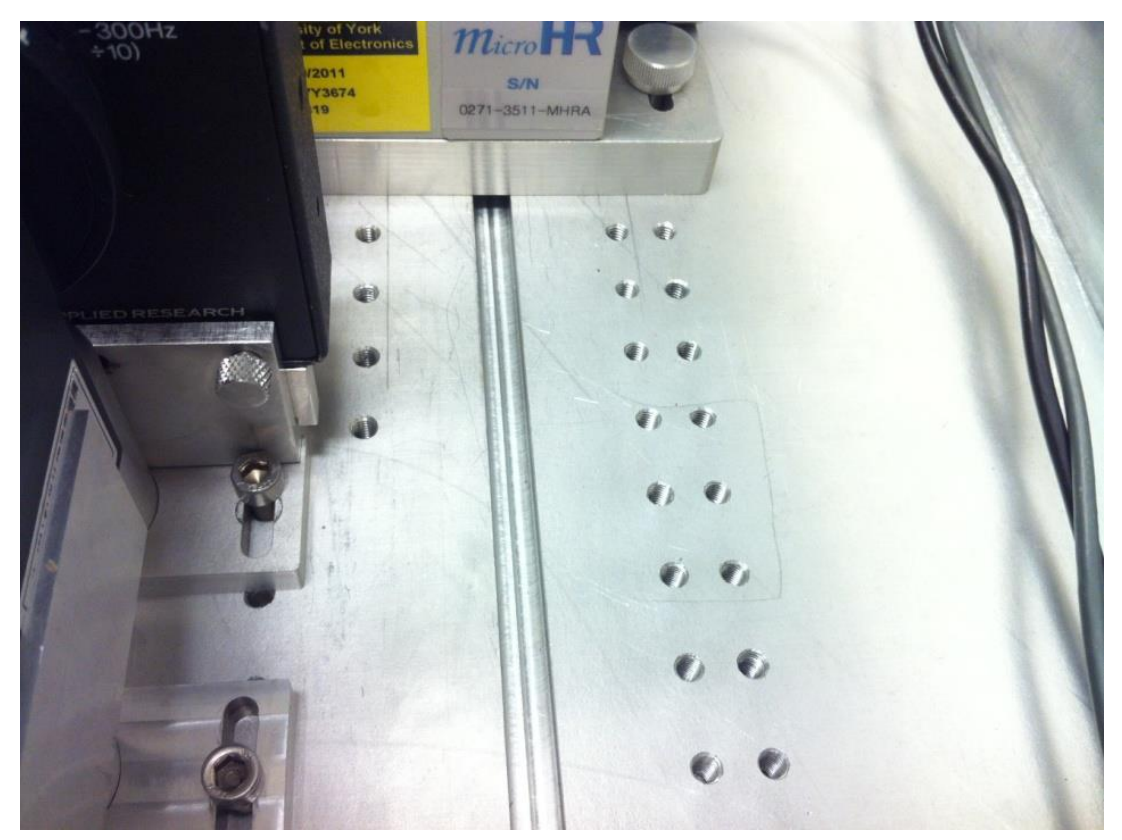

Fig. 3.19: The base of the shielding box with grooves and holes to slide and hold all components. 


\section{Chapter 4}

\section{Growth and Characterisation Techniques}

\subsection{Introduction}

This chapter is divided into two main parts. The first part is about the techniques used to grow Heusler-alloy films. These techniques are sputtering and MBE for the growth of CFS and CFAS films, respectively. The second part is about the characterisation techniques that used to study the structural and magnetic properties of the CFS and CFAS films.

\subsection{Growth Techniques}

\subsubsection{High target utilisation sputtering}

High target utilisation sputtering (HiTUS) is a unique form of a conventional magnetron sputtering that has been widely used to deposit polycrystalline thin films of different materials.

In magnetron sputtering, a target is bombarded by ions that generated in plasma as a result of electrical discharge in the inert gas environment. The target atoms are then sputtered from the target and are deposited on the substrate as a thin film [119].

The most important difference between HiTUS and the other conventional 
sputtering systems is that the plasma in HiTUS is generated remotely in a side arm and is then steered onto the target using magnetic fields. This process resulted in generation of high-density plasma at the target surface [120]. The HiTUS system consists of high vacuum (HV) chamber in which sputtering process takes place with 6 sputter targets and their associated target holder, as well as a power supply, a substrate holder and a side arm. The chamber is pumped down to a base pressure of around $5 \times 10^{3} \mathrm{~Pa}$ before introducing a controlled flow of sputtering gas, typically argon. With argon gas, the process pressure is around $3 \times 10^{3} \mathrm{~Pa}$, which is controlled by a mass flow controller. The other gases such as Nitrogen, Oxygen, Hydrogen and Helium can also be used. The sputtering targets are placed on the water-cooled holder and are usually held in a planner carousel with 6 different materials. The dimensions of the targets holder are $20 \mathrm{~cm}$ in diameter and $6 \mathrm{~mm}$ in thickness. The targets holder is rotatable with a selected $5 \mathrm{~cm}$ (2 inch) diameter target and a dark shield to allow sputtering only from the selected target. Having 6 different targets in one holder allows sputtering multilayer structures without breaking vacuum.

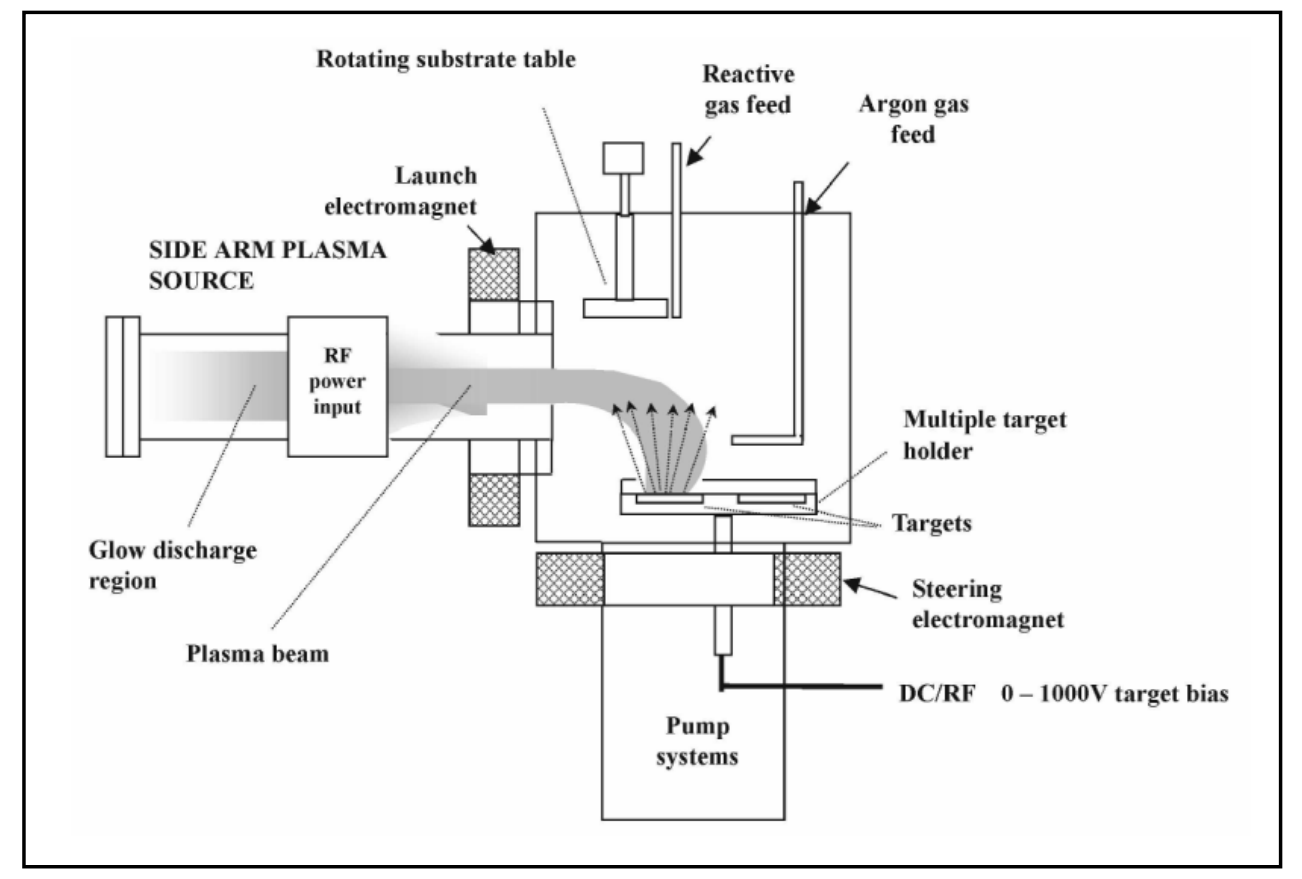

Fig. 4.1: Schematic diagram of the HiTUS system. The figure taken from [120]. 
The substrates are held in a 8-substrate holder. Different substrate sizes of up to $20 \times 20 \mathrm{~mm}$ can be used. Another advantage in the HiTUS design is that the substrate holder is placed $30 \mathrm{~cm}$ above the target. Hence, uniform sputtering can be achieved and can prevent re-sputtering to occur from the target or substrate during the deposition process. The most unique feature of HiTUS is the plasma generation in the side arm, which is attached to the main chamber. It consists of a quartz tube surrounded by a Copper coil antenna that induces a radio frequency (RF) of $13.56 \mathrm{MHz}$ and power of up to $2.5 \mathrm{~kW}$ [120]. A circular electromagnet (launch electromagnet as shown in Fig. 4.1) is fitted around the tube between the side arm and the chamber. Another electromagnet (steering electromagnet as shown in Fig. 4.1) is fitted underneath the target holder. These two electromagnets produce different magnetic fields, which allow plasma to be introduced into the main chamber and steered onto the target [121][122]. Plasma beam with high ion density of $10^{12}-10^{13}$ ions $/ \mathrm{cm}^{3}$ is used in HiTUS [123][124], which is much higher than the conventional plasma density used in the other sputtering techniques $\left(\sim 10^{10}\right.$ ions $\left./ \mathrm{cm}^{3}\right)$ [123][124], resulting in high-rate sputtering (between 0.01 and $0.09 \mathrm{~nm} / \mathrm{s})[123][124]$.

A negative DC bias voltage (from -1 to $-1000 \mathrm{~V}$ ) is applied to the target to control a deposition rate, which provides additional flexibility to HiTUS. The HiTUS system also has the other advantages, such as the ability of generating plasma beam under a wide range of pressure independently of the target types or conditions [122], and the capability of adjusting the position of the plasma beam by the alteration of the applied magnetic fields. Additionally, HiTUS has the ability of sputtering from ferromagnetic targets without using magnetic fields underneath. Therefore, it allows sputtering from thick ferromagnetic targets, which is one of the limitations of conventional sputtering systems [124]. 


\subsubsection{UHV-MBE}

MBE is a sophisticated growth technique, commonly used for growing epitaxial thin films on a (heated) single-crystal substrate under UHV environment [125-127]. It has been widely used in semiconductor industry as well as in research [125][126]. A wide variety of materials can be epitaxially deposited including semiconductors, oxides, metals and ferromagnetic materials [125][126]. The growth takes place by using atomic or molecular beam of the desired material, which are evaporated from a crucible with a source heated by electron-beam typically. The atomic or molecular beam is directed towards the substrate [125][126].

The MBE system typically consists of an UHV chamber with very low pressure $\left(<1 \times 10^{-8} \mathrm{~Pa}\right)$ and a HV load-lock chamber $\left(\sim 10^{-7} \mathrm{~Pa}\right)[127]$. It is normally equipped with in situ tools, such as low energy electron diffraction (LEED) and reflection highenergy electron diffraction (RHEED) to characterise the surfaces of the deposited films during the growth.

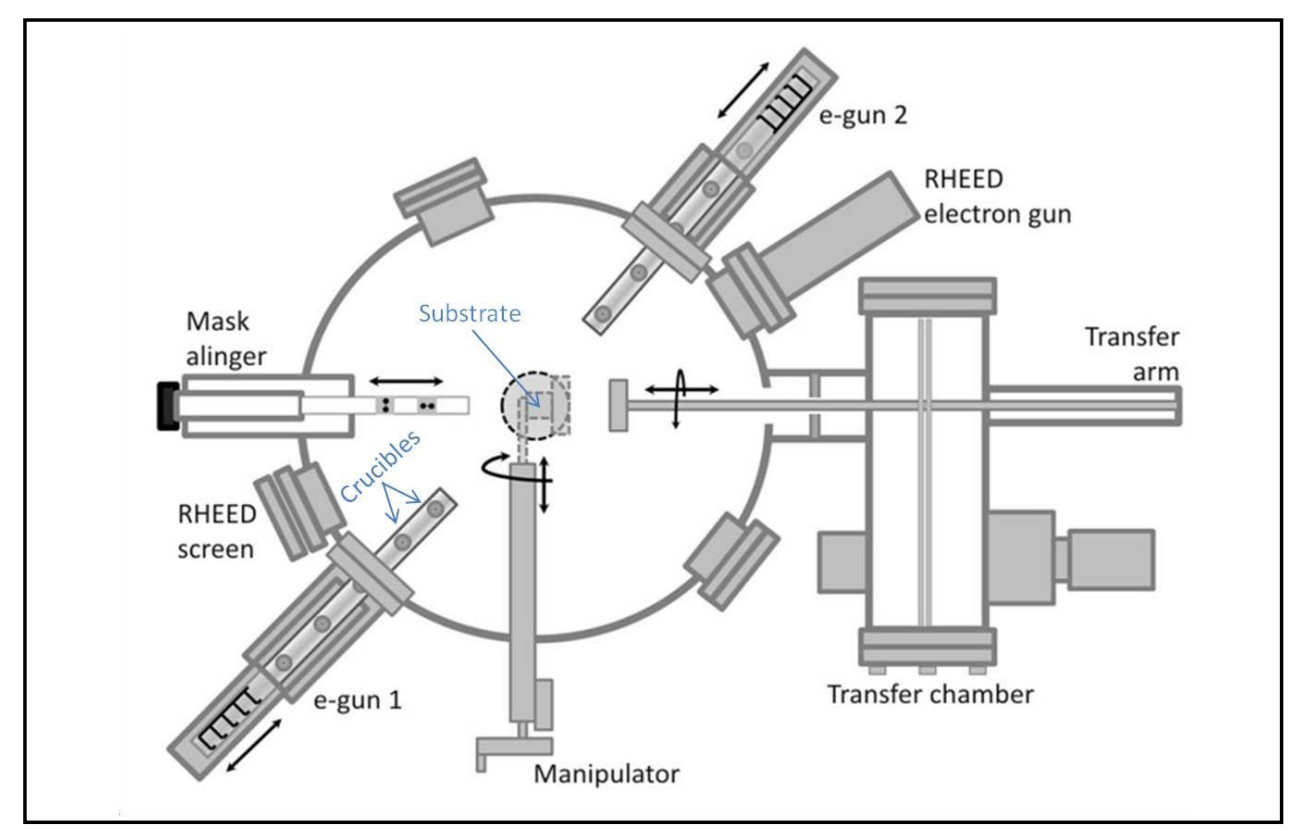

Fig. 4.2: Schematic diagram of the MBE system used in this study. The figure taken from [58].

The film thickness can be precisely controlled during the growth by adjusting the 
quartz-crystal thickness monitor. The thickness monitor has been calibrated by thickness measurements, such as cross-sectional transmission electron microscopy. The deposition rate is controlled by the temperature of the source materials. The e-guns are filled with very pure materials (typically $\geq 99.99 \%$ purity) with mechanical shutters in front of these cells to introduce the atomic or molecular beams onto the substrate while the sources are maintained above evaporator temperature. Because the shutter operation is fast enough, it allows one to grow ultra-thin layers with abrupt interfaces. In other words, the compositions can be tailor-made layer by layer with flat interfaces in a successful epitaxial process. Therefore, it is possible to fabricate innovative structures such as quantum well devices and superlattices [126] using the UHV-MBE systems. Moreover, such heterostructure (also called heteroepitaxial) films can be produced with very low contamination because of the UHV environment.

The UHV-MBE system (Eiko, HDH-10000) was used to grow CFAS films studied in this thesis (see Fig. 4.2). This system used a Knudeon cell (K-cell) and two eguns to generate molecular beams. It also used RHEED to characterise the surface crystalline structures of the film during the growth. The base pressure of the system was $<1 \times 10^{-8} \mathrm{~Pa}$ and the pressure during the deposition was $\sim 1.5 \times 10^{-7} \mathrm{~Pa}$.

A $\mathrm{MgO}(001)$ substrate was heated up to $600^{\circ} \mathrm{C}$ for about 60 minutes after loaded into the UHV-MBE system. The $23 \mathrm{~nm}$ thick CFAS film was then grown on the $\mathrm{MgO}(001)$ substrate at the deposition rate of $0.001 \mathrm{~nm} / \mathrm{s}$. In this study, $\mathrm{Co}_{2} \mathrm{Fe} \mathrm{Al}_{0.5} \mathrm{Si}_{0.5^{-}}$ alloy source is used for deposition. A thin capping layer of $\mathrm{Au}(2 \mathrm{~nm})$ was grown on top of the CFAS to prevent oxidation.

\subsubsection{Annealing}

In addition to the above two growth techniques, post-annealing treatments were used to study the thermal effect on the crystallisation of the CFS and CFAS samples. Annealing 
took place in a small tube furnace (Carbolite, model MTF10/38 A) in the clean room in the Department of Electronics at the University of York.

The furnace comprises a ceramic tube with $300 \mathrm{~mm}$ length and $38 \mathrm{~mm}$ diameter as shown in Fig. 4.3. A quartz glass tube with $500 \mathrm{~mm}$ length and $34 \mathrm{~mm}$ diameter is used to act as a chamber in the ceramic tube and to accommodate sample(s). The quartz tube has slightly smaller diameter than the ceramic tube to allow smooth insertion. The quartz tube is also connected to a cooling system. The tubes can be used up to $1000^{\circ} \mathrm{C}$ with high temperature stability of $\sim \pm 5^{\circ}$.

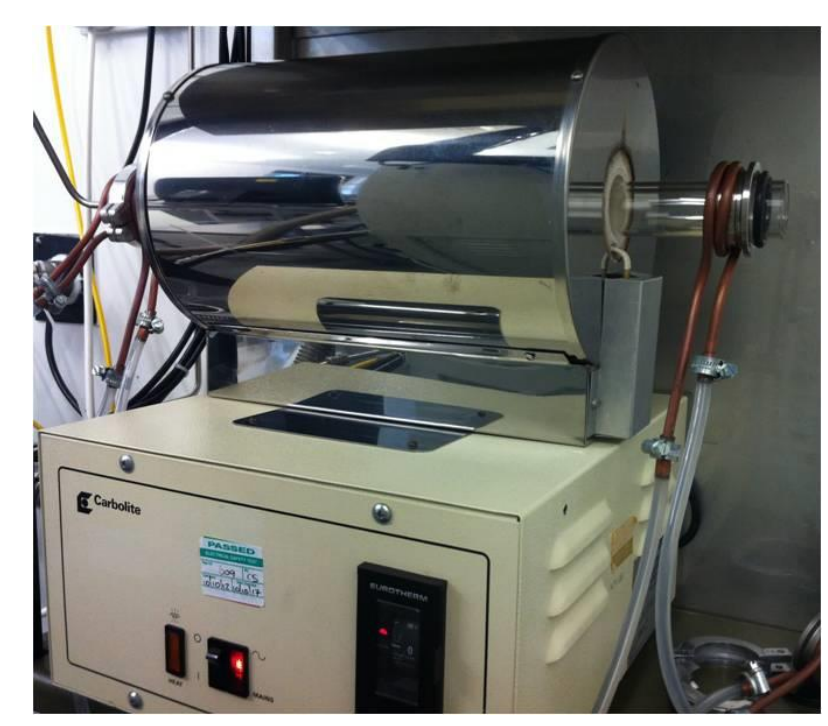

Fig. 4.3: Photograph of the furnace (Courtesy of J. Creamer).

One end of the furnace is connected to roughing (i.e., rotary) and high-vacuum (i.e., oil diffusion) pumps and the other end is connected to a gas inlet (typically Nitrogen). Both ends of the quartz tube are sealed by clamps, insulating plugs and rubber o-rings to prevent gas leakage. The temperature is controlled by a heater inside the furnace and the gas flow rate is controlled by a mass flow controller attached to the furnace.

The sample was placed in the middle of the quartz tube, which was within the 
uniformly heated zone of the furnace in order to guarantee homogeneous heat distributions over the sample. Both ends were then clamped and the $\mathrm{N}_{2}$ gas was introduced to the tube to remove any remaining particles inside the tube. The gas was kept circulating inside the tube for a few minutes, followed by setting to the required temperature and pressure (i.e., 11.33 Pa). Within a couple of minutes, the temperature was reached to the set value and the annealing was started. After completing of the annealing, the heater was turned off. It should be noted that the sample was left inside the furnace to cool down to RT for typically $1.5 \mathrm{~h}$. 


\subsection{Structural and Magnetic Characterisation}

\subsubsection{X-ray Diffraction}

$\mathrm{XRD}$ is one of the most important characterisation techniques and has been commonly used to determine the crystallographic structures of thin films. It is a fast and nondestructive technique [122]. In this technique, X-ray is scattered from atoms in adjacent crystal planes of the film at certain incident angles. If the waves are in phase with each other, a constructive interference occurs. Such constructive interference is called diffraction and it occurs at incident angles known as Bragg's angle $(\theta)$. The constructive interference of X-rays can be described by the well-known Bragg's law [128]:

$$
n \lambda=2 d \sin \theta
$$

where $n$ is an integer number, $\lambda$ is the wavelength of the X-ray, $d$ is the distance between two adjacent planes (also called $d$-spacing) and $\theta$ is the angle between the incident beam and the crystal planes. XRD can determine structural properties of the samples, such as the texture, the degree of crystallinity, the grain size and the lattice constants [122]. These properties can be obtained by analysing the XRD peak positions, intensities, width and shapes.

Various measurement methods can be used to study these properties such as inplane, out-of-plane and pole-figure measurements. In this study, $\theta-2 \theta$ (out-of-plane), $2 \theta \chi$ (in-plane), $\phi$ (in-plane) and $2 \theta_{b}$ scans as well as pole-figure measurements have been performed using an XRD system (Rigaku, SmartLab). Figures 4.4(a) and (b) show the geometrical layouts of the conventional $\theta-2 \theta, \varphi$ and $2 \theta \chi$ measurements. In the outof-plane measurement (e.g., $\theta-2 \theta$ scan), the sample is placed horizontally, and the incident X-ray and the detector move simultaneously with maintaining their angles to be $\theta$ and $2 \theta$, respectively. The scattering vector is pointed perpendicular to the film surface.

In addition, two in-plane measurements have been conducted; $2 \theta \chi$ and $\varphi$ scans. 
For the $\phi$ scan, the sample is placed horizontally and rotates in the stage plane with the incident X-ray and the detector to be fixed at a small angle (typically $0.4^{\circ}$ ). For the $2 \theta \chi$ scan, the sample is placed horizontally with the incident X-ray and the detector to move simultaneously at a fixed angle (typically $0.4^{\circ}$ ). For both $2 \theta \chi$ and $\phi$ scans, the scattering vector is in parallel to the film surface. The $2 \theta_{b}$ scan has also been conducted to determine the detailed crystalline structures [see Fig. 4.4(c)]. The sample is tilted at an angle (i.e., $\alpha$ ), which can be extracted from the position of the diffraction spots appeared in the pole-figure scan. The $2 \theta_{b}$ scan can reveal the origin of a peak by comparing the position of the corresponding peak in the $\theta-2 \theta$ scan. If the positions of the peak in the $2 \theta_{b}$ and $\theta-2 \theta$ scans match, the peak can be attributed to the reflection from a determined crystalline structure. In the pole-figure measurement, the sample is placed horizontally and rotates $360^{\circ}$ in the stage plane with the incident X-ray and the detector to move simultaneously at an angle $0 \leq \alpha \leq 90^{\circ}$ [see Fig. 4.4(d)]. Thus, the diffracted intensity data covers the whole sample and therefore gives more information about the crystalline structures. 

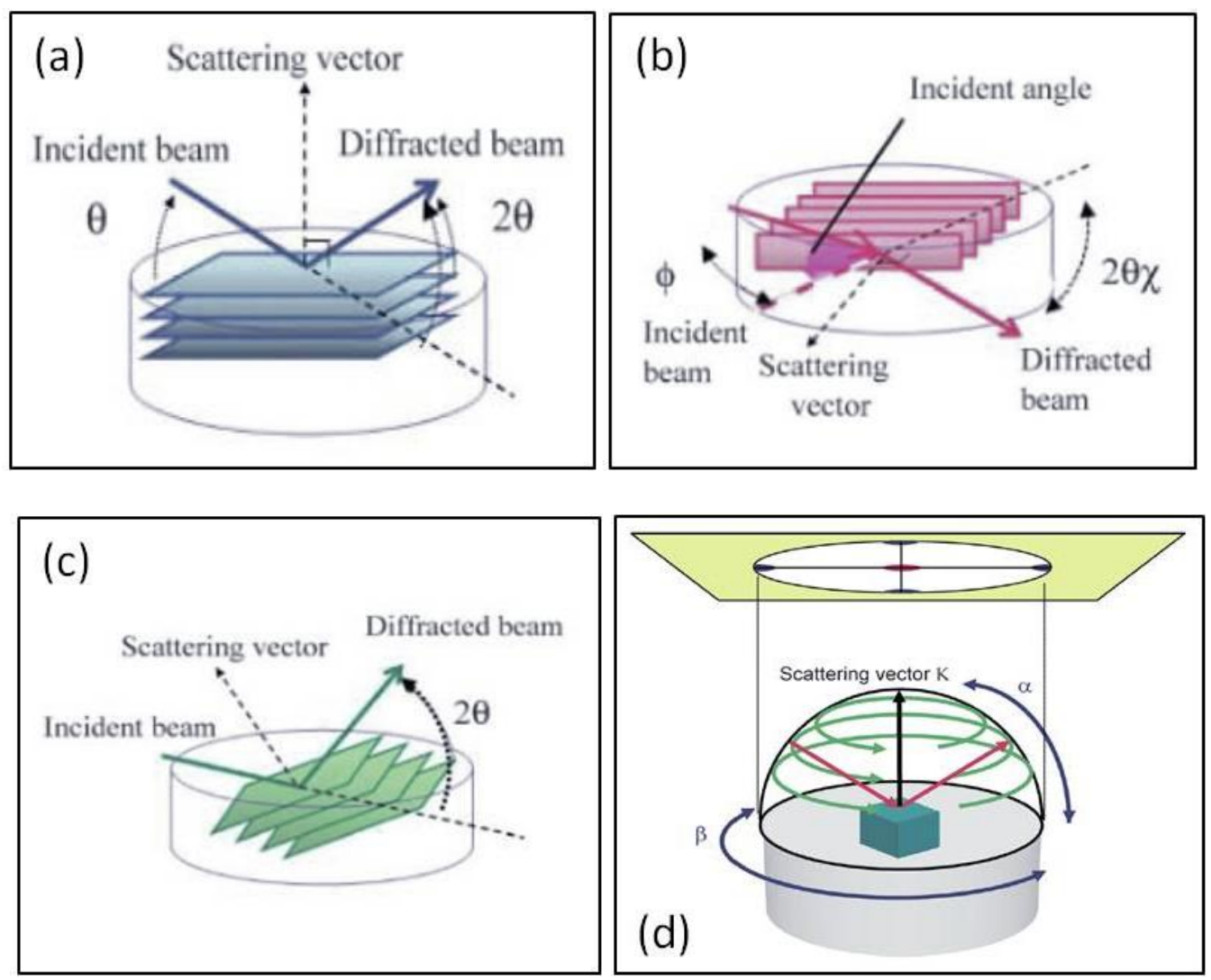

Fig. 4.4: Configurations of (a) $\theta-2 \theta$ (b) $\phi$ and $2 \theta \chi$ (c) $2 \theta_{b}$ and (d) pole figure scans. The figures taken from [129][130].

\subsubsection{Transmission Electron Microscope}

TEM is an analytical tool to directly visualise an atomic structure with the resolution of $\sim 0.1 \mathrm{~nm}$ and to provide chemical information of a very thin $(\leq 100 \mathrm{~nm})$ specimen [131]. TEM characterises a high-energy electron beam transmitted through the thin specimen to generate a nano-scale structural image. TEM also allows researchers to examine the chemical compositions of the materials using energy dispersive X-ray spectroscopy (EDX) and electron energy-loss spectroscopy (EELS). These properties can be fedback to control and to optimise the growth of the specimen. TEM usually consists of three primary parts located in a vacuum column [132]; (i) an electron gun, where the electron-beam is emitted and directed towards the specimen, (ii) a pair of electromagnetic condenser lenses and condenser apertures to focus the electron-beam, 
and (iii) imaging systems, consisting of electromagnetic lenses and a fluorescent screen or charge-coupled device (CCD) camera that reconstruct an image for recording.

There are two basic TEM imaging modes known as the diffraction pattern and the conventional image modes, both of which are as shown in Fig. 4.5. By adjusting the focal length of the intermediate lens [see Fig. 4.5(b)], the diffraction and imaging modes can easily be switched using the same TEM.

In the diffraction mode, the back focal plane functions as an objective plane for the intermediate lens, resulting that a diffraction pattern can be collected via the CCD camera or fluorescent screen [see Fig. 4.5(a)]. This is similar to the diffraction patterns obtained by X-ray. When a sample has epitaxial or single-crystal structure, diffraction spots can be observed on the monitor or fluorescent screen. However, if a sample is polycrystalline, diffraction patterns become circular rings. An amorphous sample presents sequences of diffuse voids. On the other hand, for the imaging mode, the image plane of the objective lens functions as an objective plane for the intermediate lens, resulting that a crystalline image appears on the monitor [see Fig. 4.5(b)].

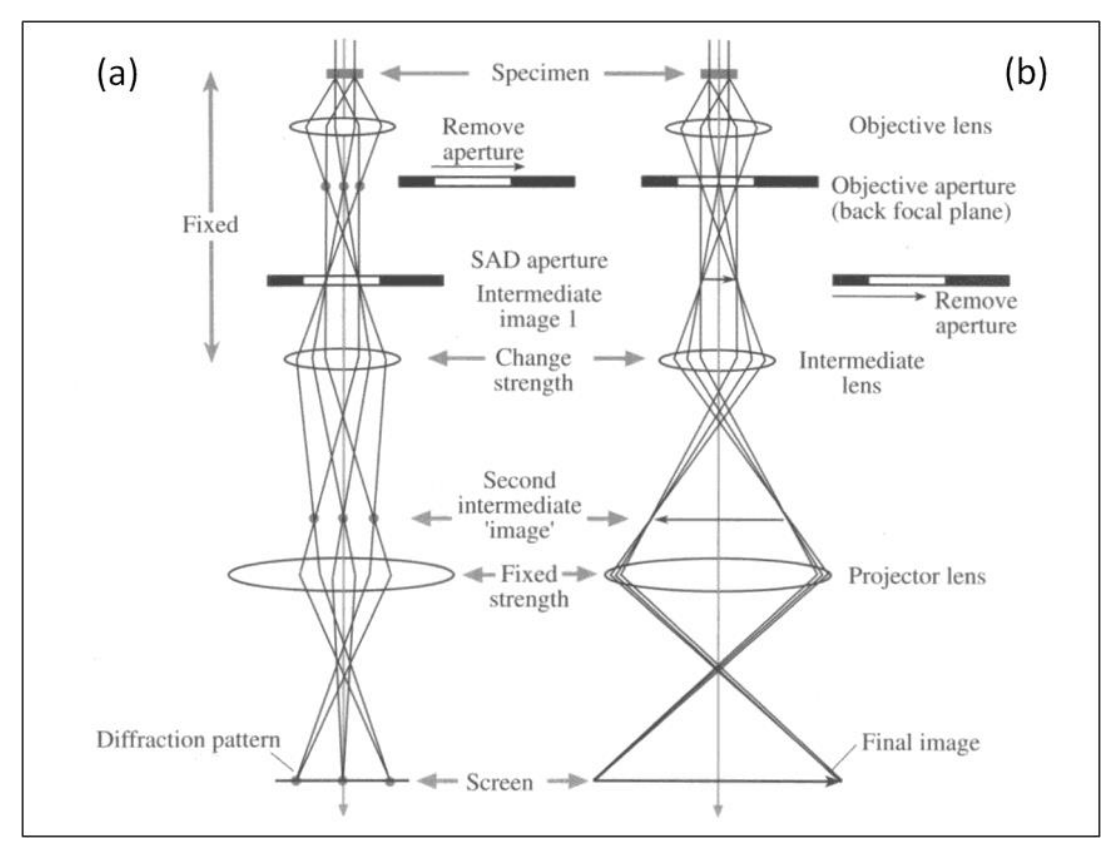

Fig. 4.5: Schematic diagrams of the two basic operation modes of TEM: (a) the diffraction mode and (b) the imaging mode. The figure taken from [133]. 
However, there are several restrictions to use these methods, including welldefined separation between the individual atomic columns, uniform thickness of a specimen and distorted electron diffraction by disordered structures. Therefore, switching between the imaging and diffraction modes allows a wider range of TEM analysis to overcome these restrictions. Moreover, TEM can give information about the atomic disorder and strain of a sample. XRD does not provide information if atoms swap their locations (i.e., $\mathrm{X}$ with $\mathrm{Y}$ ) because the $\mathrm{X}$-ray is scattered from atomic positions regardless of their element. However, XRD provides some indication about the atomic disorder if the expected peaks do not appear at the expected angles. 


\subsubsection{Vibrating Sample Magnetometer}

Magnetic characterisations in this study have been made using a VSM. VSM is a very common technique to measure the magnetic properties of different types of materials. A block diagram of VSM is shown in Fig. 4.6.

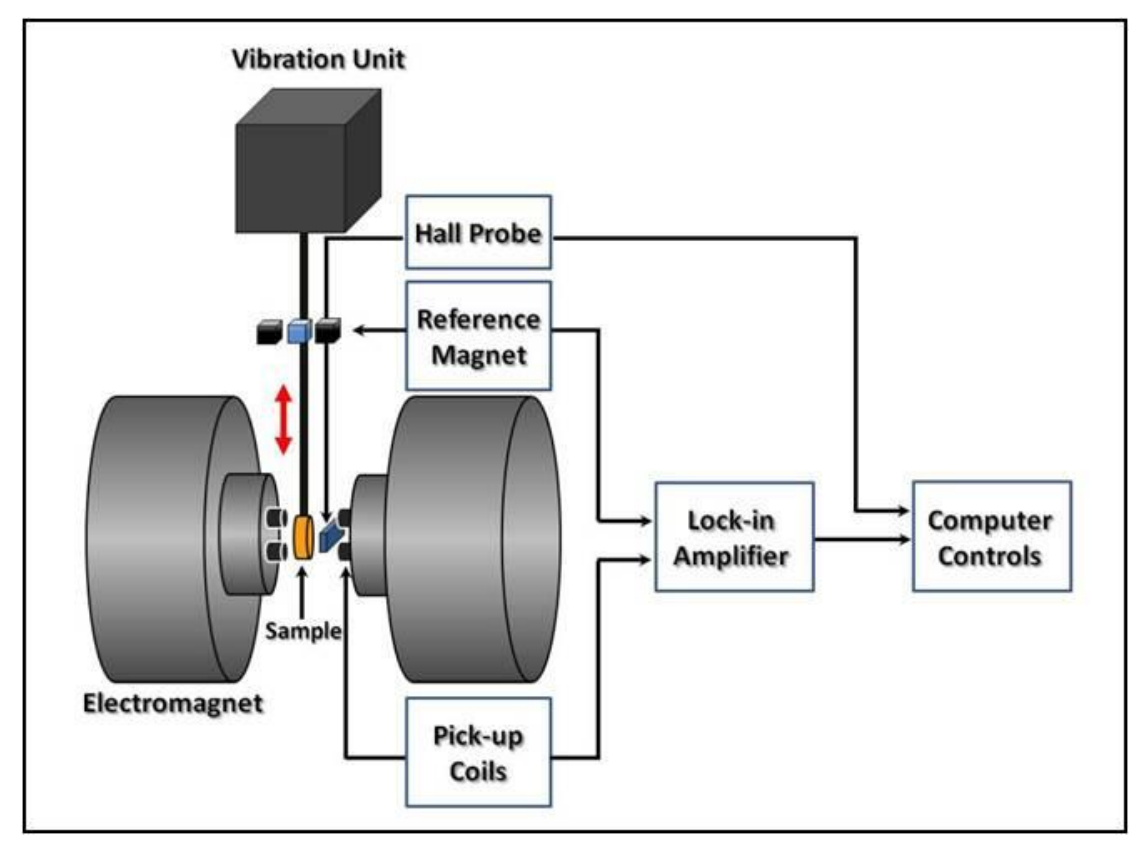

Fig. 4.6: Block diagram of the VSM (ADE, model 10). The figure taken from [134].

Faraday's law is the primary principle for VSM. The electromagnetic induction can be represented in Equation 4.2 [135].

$$
\varepsilon=-N \frac{d \emptyset_{m}}{d t}
$$

where $\varepsilon$ represents the induced voltage, $\phi_{\mathrm{m}}$ represents the magnetic flux that the specimen generates into the detection coils and $N$ is the number of turns of the coils. VSM is based on the measurement of the alteration of the magnetic flux that occurs when the sample is vibrated between the detection coils under an application of a magnetic field $(H)$ produced by an electromagnet. The total magnetic flux generated by the sample is dependent upon the following parameters; $H$, the sample magnetisation 
$(M)$ and the cross-sectional area of the coil $\left(A_{\mathrm{c}}\right)$. The total magnetic flux is hence given by Equation 4.3 [135]:

$$
\emptyset_{m}=B_{m} \cdot A=(H+M) \cdot A
$$

where $B_{\mathrm{m}}$ represents the magnetic flux density. When the sample moves inside the coil, it generates electromotive force (e.m.f.) with its magnitude proportional to the magnetisation of the sample. This is according to Faraday's law of induction, providing the induced voltage by Equation 4.4 .

$$
\int \varepsilon \cdot d t=-N A \cdot M
$$

The induced voltage is then fed into a lock-in amplifier. Here, its output signal is proportional to the magnetisation of the sample.

Calibration is essential to carry out accurate measurements. Palladium (Pd) is commonly used for the calibration owing to its large and linear magnetic susceptibility [136]. In this study, VSM (ADE, model 10) has been used. 


\section{Chapter 5}

\section{Heusler Band-Gap Measurements on}

\section{Polycrystalline $\mathrm{Co}_{2} \mathrm{FeSi}$ Films}

\section{$5.1 \quad$ Introduction}

CFS is a full-Heusler alloy and is considered to be one of the most promising candidates for future spintronic applications [62]. It exhibits almost the highest $T_{\mathrm{C}}$ and the highest magnetic moment $(m)$ among all Heusler alloys as well as HMFs as listed in Table 5.1 [62][63]. These properties are desirable from the application point of view. $T_{\mathrm{C}}$ of the CFS has been calculated to be $1185 \mathrm{~K}$ [4] and is close to the experimentally reported value of $(1100 \pm 20) \mathrm{K}[4] . T_{\mathrm{C}}$ is also below the melting point $(1520 \pm 5) \mathrm{K}$ [62]. The magnetic moment of the CFS has been reported to be $(5.97 \pm 0.05) \mu_{\mathrm{B}} / \mathrm{f}$.u. (Bohr magneton per formula unit) at $5 \mathrm{~K}$ and $6 \mu_{\mathrm{B}} /$ f.u. at $0 \mathrm{~K}$ by an extrapolation [62][63]. This value, i.e., $6 \mu_{\mathrm{B}} /$ f.u., agrees exactly with the predicted value from the generalised SlaterPauling curve [62][63].

$P$ for an $L 2_{1}$-ordered CFS film has been measured at $4.2 \mathrm{~K}$ to be $(49 \pm 2 \%)$ by the point contact Andreev reflection (PCAR) technique [64]. This value is in good agreement with the $P$ value of $\sim 0.5$ that was derived from a TMR ratio of a MTJ with a 
CFS electrode using Julliere's formula [64]. This is smaller than the expected halfmetallicity but large enough with possible surface/interface atomic disordering. Therefore, the CFS has been widely used in the MTJs as one of the main applications to increase their TMR ratios. The CFS can be fabricated in a film form by sputtering [137][138] and MBE deposition [139], and in a bulk form by arc melting [2] and rapid thermal annealing (RTA) [140]. Importantly, the band-gap has been calculated to be between 100 and $780 \mathrm{meV}$ using different versions of the density functional theory (DFT) calculation method, which is one of the largest among the Heusler alloys as discussed in Section 2.2 in Chapter 1. It was predicted to be $100 \mathrm{meV}$ using DFT calculations [65], $146 \mathrm{meV}$ using generalised gradient approximation (GGA) calculations [66], $400 \mathrm{meV}$ using local density approximation (LDA) [67] and $780 \mathrm{meV}$ using local density approximation with electron-electron Coulomb interactions (LDA + $U$ [66][141]. These large variations caused by using different calculation methods and input parameters. Our estimated band-gap value (i.e., $96 \mathrm{meV})$ is close to the calculated value using DFT (i.e., $100 \mathrm{meV}$ ) [65]. Moreover, our band-gap measurements have shown good reproducibility with only small variations between the samples grown on $\mathrm{MgO}(001)$ and Si substrates. 
Table 5.1: List of magnetic properties of the CFS obtained experimentally and theoretically.

\begin{tabular}{|c|c|c|c|c|}
\hline $\begin{array}{c}\text { Magnetic } \\
\text { moment }(m) \\
{\left[\mu_{B} / \text { f.u. }\right]}\end{array}$ & $\begin{array}{c}\text { Saturation } \\
\text { magnetisation } \\
(M \mathbf{s}) \\
{\left[\mathrm{emu} / \mathrm{cm}^{3}\right] /} \\
\mu_{B} / \text { f.u. }\end{array}$ & $\begin{array}{c}\text { Spin } \\
\text { polarisation }(P)\end{array}$ & $\begin{array}{c}\text { Band-gap }(\boldsymbol{E} \mathbf{g}) \\
{[\mathrm{meV}]}\end{array}$ & $\begin{array}{c}\text { Curie } \\
\text { Temperature } \\
\left(T_{\mathrm{C}}\right) \\
{[\mathrm{K}]}\end{array}$ \\
\hline $\begin{array}{c}\text { Exp. } 5.97 \pm 0.05 \\
\text { (at } 5 \mathrm{~K}) \\
{[62][63]}\end{array}$ & $\begin{array}{c}\sim 860 / 4.2 \\
{[68]}\end{array}$ & $\begin{array}{c}49 \pm 2 \%(\mathrm{PCAR}) \\
\text { at } 5 \mathrm{~K}[64]\end{array}$ & 100 (DFT) [65] & $1100 \pm 20[4]$ \\
\hline \multirow[t]{4}{*}{$\begin{array}{c}\text { Cal. } 6 \\
(\text { at } 0 \mathrm{~K}) \\
{[62][63]} \\
\end{array}$} & $\begin{array}{c}\sim 850 / 4.1 \\
{[137]}\end{array}$ & $\begin{array}{c}\sim 58 \%(\mathrm{GGA}) \\
{[66]}\end{array}$ & 146 (GGA) [66] & 1185 [4] \\
\hline & $\begin{array}{c}\sim 1130 / 5.4919 \\
{[69]}\end{array}$ & $\begin{array}{c}\sim 50 \%(\mathrm{MTJ}) \\
{[64]}\end{array}$ & 400 (LDA) [67] & \\
\hline & $\begin{array}{c}\text { 5.7-6 } \mu_{B} / \text { f.u. } \\
{[70]}\end{array}$ & $\begin{array}{c}61 \% \text { (MTJ) } \\
\text { at RT } \\
{[142]}\end{array}$ & $\begin{array}{c}780(\mathrm{LAD}+U) \\
{[66][141]}\end{array}$ & \\
\hline & & $\begin{array}{c}\sim 93 \% \\
(\mathrm{LDA}+U)[12]\end{array}$ & & \\
\hline
\end{tabular}

\subsection{Film growth}

Polycrystalline $\mathrm{Co}_{2} \mathrm{FeSi}$ thin films were deposited on naturally oxidised $\mathrm{Si}$ and $\mathrm{MgO}(001)$ substrates by sputter deposition (Plasma Quest, HiTUS). The Si and $\mathrm{MgO}(001)$ substrates were cleaned by acetone in ultrasonic bath for $45 \mathrm{~s}$. before loading them into the HiTUS system. The substrates were then loaded into the HiTUS system for the CFS deposition. The base pressure of the system was $5 \times 10^{-8} \mathrm{~Pa}$ and the sputtering pressure was $3 \times 10^{-4} \mathrm{~Pa}$. The plasma was generated by an RF field at a frequency of $13.56 \mathrm{~Hz}$ and was steered onto a 2 inch diameter target. The films were deposited at a rate of $0.07 \mathrm{~nm} / \mathrm{s}$ using a steering bias voltage of $990 \mathrm{~V}$. The dimensions of the $\mathrm{Co}_{2} \mathrm{FeSi}$ films were $5 \times 5 \mathrm{~mm}^{2}$ with the thickness of $23 \mathrm{~nm}$. A $2 \mathrm{~nm}$ capping layer of $\mathrm{Ru}$ was then deposited to prevent oxidation. The films were then annealed ex situ at $400^{\circ} \mathrm{C}$ for up to 6 hours in steps of 1.5 hours. During annealing the films were set in a 
quartz glass tube and placed inside the furnace (Carbolite, model MTF10/28 A) with nitrogen gas flowing at a rate of $100 \mathrm{sccm}$ with a corresponding pressure of $\sim 2.7 \mathrm{kPa}$. After the annealing, the films were kept inside the furnace to cool down to RT for $\sim 4 \mathrm{~h}$.

\subsection{Film characterisation}

The films grown on the $\mathrm{MgO}(001)$ substrates were first characterised structurally by $\mathrm{XRD}$ with a $\mathrm{Cu} \mathrm{K} \alpha$ source. The evolution of crystallisation of the $\mathrm{Co}_{2} \mathrm{FeSi}$ films was measured before and during post-annealing at $400^{\circ} \mathrm{C}$ for up to 6 hours as shown in Fig. 5.1. The principle CFS(220) peak was observed at $2 \theta \sim 45^{\circ}$ for the 3 and $6 \mathrm{~h}$ annealed film. The (220) peaks increased their intensity with increasing annealing time. One would expect to see a superlattice CFS(200) peak for the disordered phase (B2 structure) and an additional CFS(111) diffraction peak for a fully ordered film $\left(L 2_{1}\right.$ structure) [139]. This indicates that the films have at least the $A 2$ phase after the annealing for 3 hours. A minor peak at $2 \theta \sim 43^{\circ}$ was appeared after 6 hours annealing which may be related to phase segregation as reported before [138][143]. Additional analysis using TEM revealed that these films form the $B 2$ and $L 2_{1}$ phases as shown in Fig. 5.2 [143][144]. Here, similar crystalline ordering was found in the films grown on the Si substrates and SiN TEM grids. As shown in Fig. 5.2(a), the films showed polycrystalline nature [as confirmed by the rings in the electron diffraction pattern in Fig. 5.2(a)] with their grain size of 72 to $374 \mathrm{~nm}$ [144]. Even so, they were found to form the $B 2$ phase [143]. Forming the atomic structure (i.e., $\left.L 2_{1}\right)$ during sample growth can be determined by XRD or TEM (as detailed in Sections 4.3.1 and 4.3.2).

The films were characterised magnetically using a VSM. Hysteresis loops were measured at RT as shown in Fig. 5.3. Coercivity of the films was measured to be $\sim 25$ Oe and 480 Oe under a field applied in-plane and perpendicular to the films, respectively. These values were small enough for the films to be saturated by a 
permanent magnet as described in Chapter 3. These loops have the squareness of 0.89 and 0.98 for under a field applied in-plane and perpendicular to the films, respectively. The saturation magnetisation $\left(M_{\mathrm{s}}\right)$ has a correlation with the crystallinity of a sample. $M_{\mathrm{s}}$ was obtained from the $M-H$ hysteresis loop as the highest value in units of emu. This value was then divided on the volume of the sample to be emu/cm ${ }^{3}$ in order to be comparable to other samples. The volume of the sample was calculated by multiplying length by width to obtain the area, and then multiplying the area by the thickness. $M_{\mathrm{s}}$ of CFS films grown on the $\mathrm{MgO}(001)$ substrate was found to be $(960 \pm 96) \mathrm{emu} / \mathrm{cm}^{3}$, which is similar to the previously reported value of $\sim 860 \mathrm{emu} / \mathrm{cm}^{3}$ [68]. These values were $70 \%$ of the CFS bulk value [68]. It could also be expressed in $\mu_{B} /$ f.u. to be $4.2 \mu_{B} /$ f.u., which was again $\sim 70 \%$ of the bulk value reported $\left(6 \mu_{B} /\right.$ f.u. $)\left(1 \mu_{B}=205 \mathrm{emu}\right)$. Similarly, $M_{s}$ for the CFS/Si was measured to be $(634 \pm 72) \mathrm{emu} / \mathrm{cm}^{3}$, which was $\sim 52 \%$ of the bulk value [63]. These values also confirm the crystalline ordering of these films.

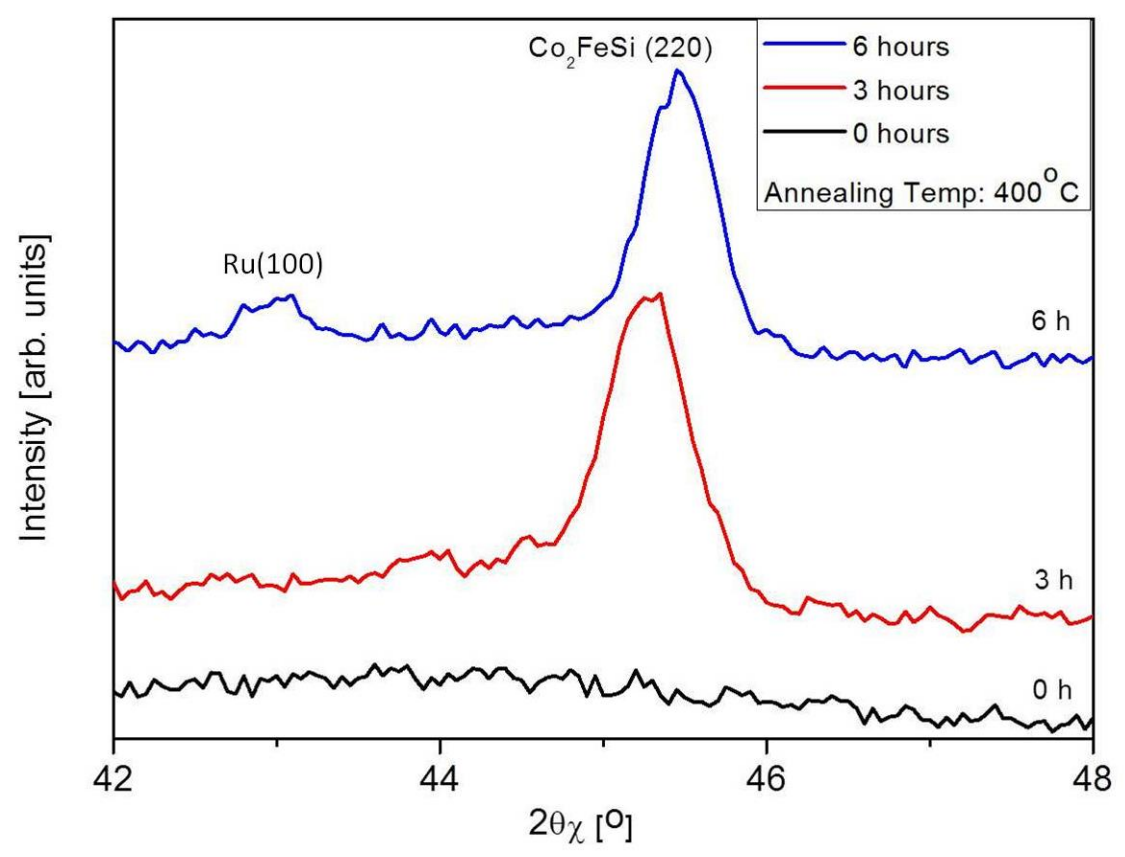

Fig. 5.1: XRD intensities for the $\mathrm{Co}_{2} \mathrm{FeSi}$ films grown on $\mathrm{MgO}(001)$ after post-annealing at $400^{\circ} \mathrm{C}$ for $0-6$ hours. 

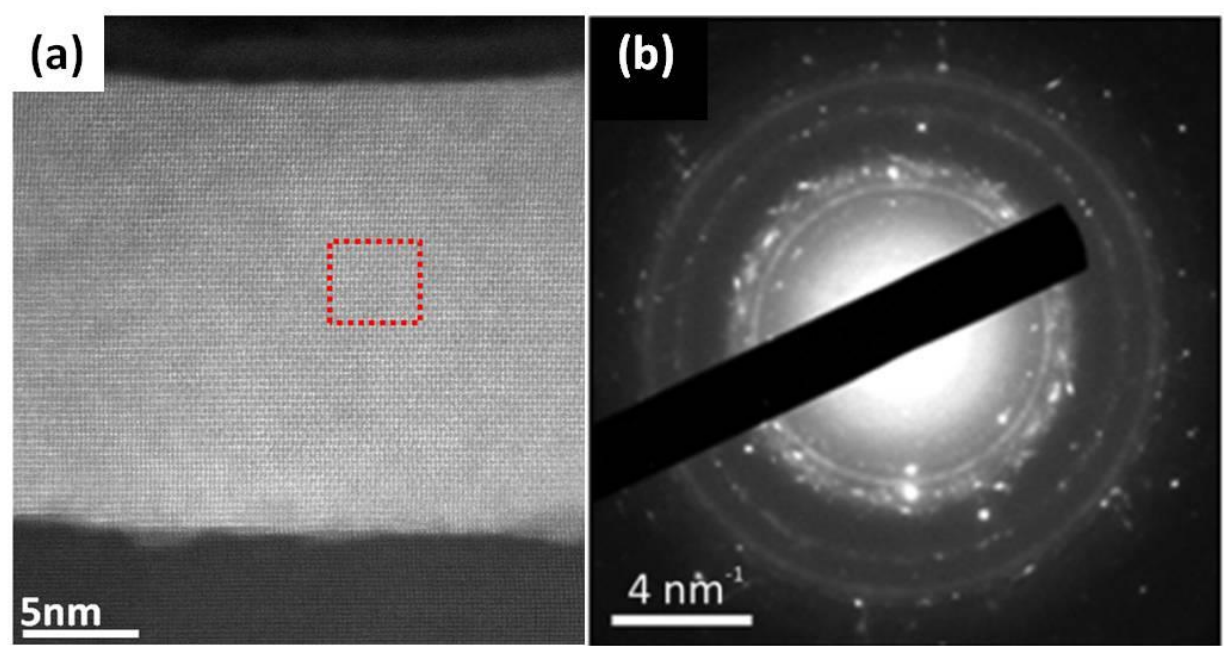

Fig. 5.2: (a) TEM image [144] and (b) the corresponding electron diffraction patterns for the $\mathrm{Co}_{2} \mathrm{FeSi}$ films grown on $\mathrm{MgO}(001)$. The figure taken from [143].

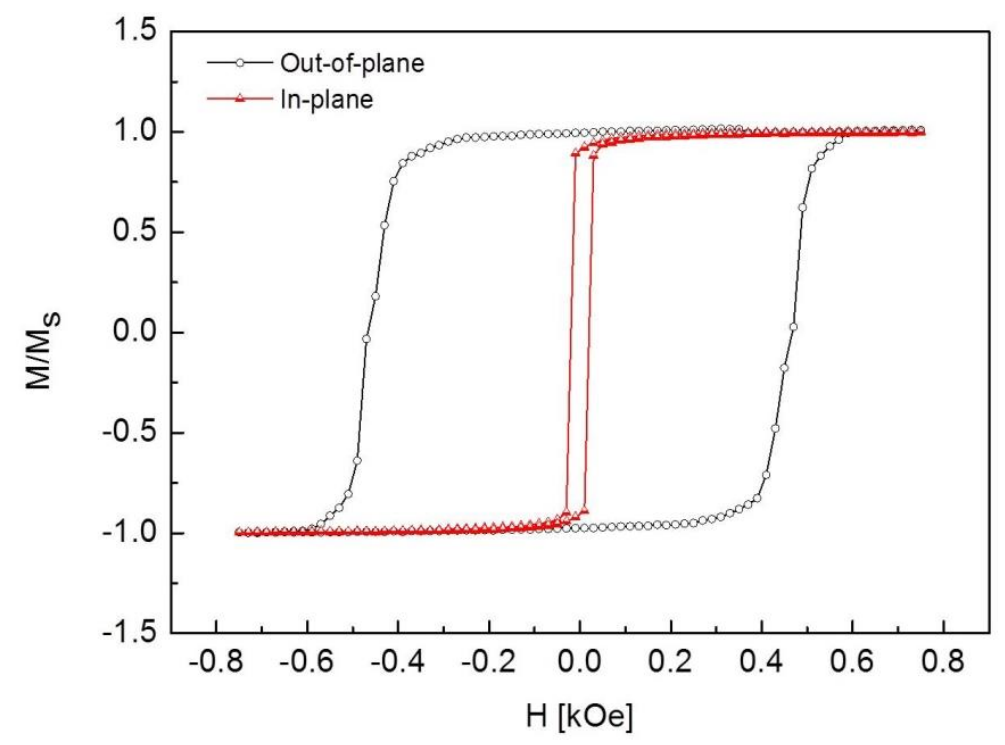

Fig. 5.3: Hysteresis loops for the as-deposited $\mathrm{Co}_{2} \mathrm{FeSi}$ film grown on $\mathrm{MgO}(001)$ under a magnetic field applied perpendicular and parallel to the film.

\subsection{Band-gap measurements}

The band-gap measurements were conducted by introducing the circularly polarised IR light $(h v)$ with energy matching to the range of $E_{\mathrm{g}}$ of the CFS films. The IR light was introduced onto the surface of the films, while the magnetic field was applied onto the films from the back. 
The reflected intensity from the as-deposited films was measured without applying a magnetic field, followed by measurements under magnetic fields with the Nand S-pole upwards over the wavelength range between 3 and $16 \mu \mathrm{m}(78 \leq h v \leq 413$ $\mathrm{meV}$ ). This range was covered by the two sets of measurements due to the spectral limitation of two gratings used as discussed in Chapter 3. The first measurement range was between 3 and $9.5 \mu \mathrm{m}$ and the second was between 9.5 and $16 \mu \mathrm{m}$ (see Figs. 5.4 and 5.5, respectively). The intensity profiles of the three measurements showed a similar trend in the entire wavelength range, which confirms that these individual intensity measurements were reproducible. However, in all of the band-gap measurements, there were dips at 4.5 and $7.2 \mu \mathrm{m}$ [see Fig. 5.4(a)], which are attributed to the artifact from the IR light source as both data for $\mathrm{N}$ - and S-pole upwards show the same behaviour as seen in Figs. 5.4(b) and 5.5(b). Therefore, these dips were not involved in the determination of the films band-gap. Figs. 5.4(b) and 5.5(b) show the normalised intensities for as-deposited $\mathrm{Co}_{2} \mathrm{FeSi}$ films without a field and with fields with $\mathrm{N}$ - and S-pole upwards in the wavelength range between 3 and $9.5 \mu \mathrm{m}$ and between 9.5 and $16 \mu \mathrm{m}$, respectively.

It is expected in these measurements to see an absorption of the IR light at a wavelength in one of the field directions; either in the $\mathrm{N}$ - or S-pole upwards. The expected absorption is due to the split band structure of the Heusler alloy films, which contains a metallic band structure for the majority spins and a semiconducting band structure with a gap at $E_{\mathrm{F}}$ for the minority spins. As a result, the metallic band does not absorb the IR light due to the fully occupied states at $E_{\mathrm{F}}$. In other words, because of the absence of an energy gap in the vicinity of $E_{\mathrm{F}}$, the light is reflected in the entire wavelength range. On the other hand, the semiconducting band does absorb the IR light when its energy matches with the energy gap of the Heusler alloy films. Based on the selection rule $\left(\Delta m_{j}= \pm 1\right)$, right circularly polarised light excites electrons from valence 
band to conduction band with one spin direction $\left(\sigma^{+}\right)$and the left circularly polarised light $\left(\sigma^{-}\right)$excites the electrons with the opposite spin direction [145]. In our set-up, the light polarisation direction is fixed as detailed in Chapter 3 and the magnetic field is reversed for each measurement as above. Therefore, the absorption is expected to be observed only in one of the field applications.

The absorption of the IR light in one of the field directions was expected to be seen as a dip-like signal. This is because the photons would be reflected when they have energy less than the gap energy and the photons would be absorbed when their energy matches with the gap, reducing the reflection intensity. The photons would also be reflected when they have energy larger than the gap energy. There would be no photon absorption in the opposite field direction. Such distinctive behaviour should be the criteria to identify the band-gap in this study.

Due to the thermal effect, which widens the definition of the band-gap edges, the band-gap is expected to become narrower at RT. This means that the top of the valence band becomes closer to the bottom of the conduction band with broadened energy band edges. This may broaden the measured dip in the reflected signals. The dip becomes apparent by taking the difference in the reflected signals between the two field applications. Therefore, the band-gap is determined by taking the intensity differences between measurements with the $\mathrm{N}$ - and S-pole upwards, allowing us to have closer view at the differences. 

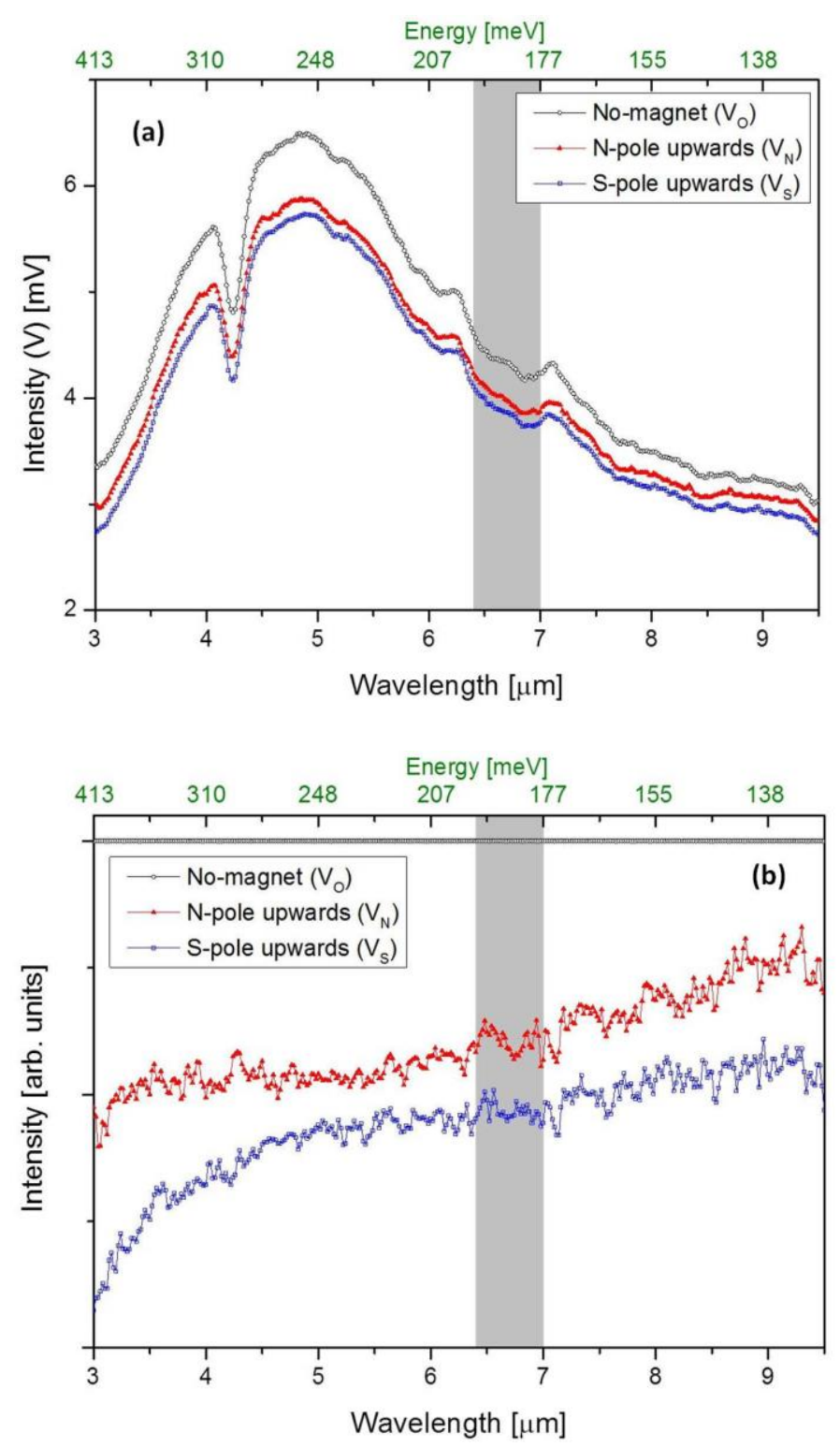

Fig. 5.4: (a) Measured intensities and (b) normalised intensities for as-deposited $\mathrm{Co}_{2} \mathrm{FeSi}$ films without a field and with fields with $\mathrm{N}$ - and S-pole upwards in the wavelength range between 3 and $9.5 \mu \mathrm{m}$. 

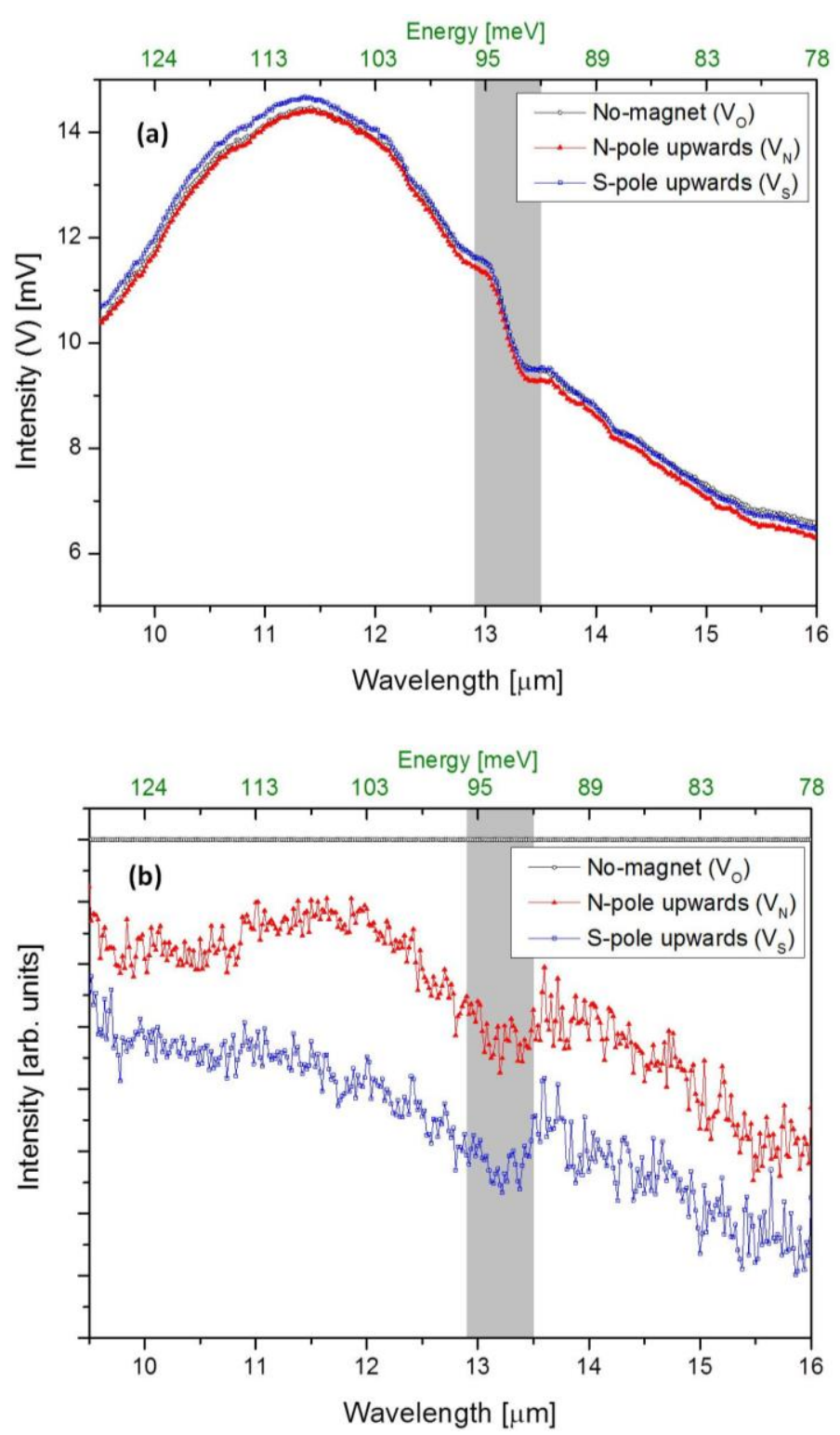

Fig. 5.5: (a) Measured intensities and (b) normalised intensities for as-deposited $\mathrm{Co}_{2} \mathrm{FeSi}$ films without a field and with fields with $\mathrm{N}$ - and S-pole upwards in the wavelength range between 9.5 and $16 \mu \mathrm{m}$.

\subsection{1. $\mathrm{Co}_{2} \mathrm{FeSi} / \mathrm{Si}$}

Silicon ( $\mathrm{Si}$ ) is very important in conventional electronics and very attractive for spintronic applications owing to its long spin diffusion length [146]. In this study, Si substrates are used to grow Heusler-alloy films for the first set for the band-gap measurements. The CFS films were deposited on $\mathrm{Si}_{/} \mathrm{SiO}_{2}$ substrates. The CFS has a bulk and thin film lattice constant of $0.5647 \mathrm{~nm}$ [140] and $0.5636 \mathrm{~nm}$ [140], 
respectively and $\mathrm{Si}$ has a lattice constant of $0.543 \mathrm{~nm}$ [147], giving a small lattice mismatch to the Si substrates of $\sim 4 \%$ [146]. The intensity signals were measured for the as-deposited CFS films without applying a magnetic field and with the fields with the N- and the S-pole upwards as shown in Fig. 5.6. The three signals were similar with some minor differences at the wavelengths ranging between 10 and $11 \mu \mathrm{m}$, between 12.8 and $13.1 \mu \mathrm{m}$ and between 15 and $16 \mu \mathrm{m}$. The detailed differences between the signals taken with the fields with the $\mathrm{N}$ - and S-pole upwards were evaluated as shown in Fig. 5.7(a). There was a dip only for the signal with the S-upwards fields, which was apparent in the difference signal $\left(\Delta V=V_{\mathrm{N}}-V_{\mathrm{S}}\right)$ as shown in Fig. 5.7(b). The difference signal revealed the dip at the wavelength of $12.9 \mu \mathrm{m}$ with the amplitude of $96 \mu \mathrm{V}$. This dip was expected to be related to the band-gap of ( $96 \mathrm{meV})$ for the CFS films grown on $\mathrm{Si} / \mathrm{SiO}_{2}$ substrates.

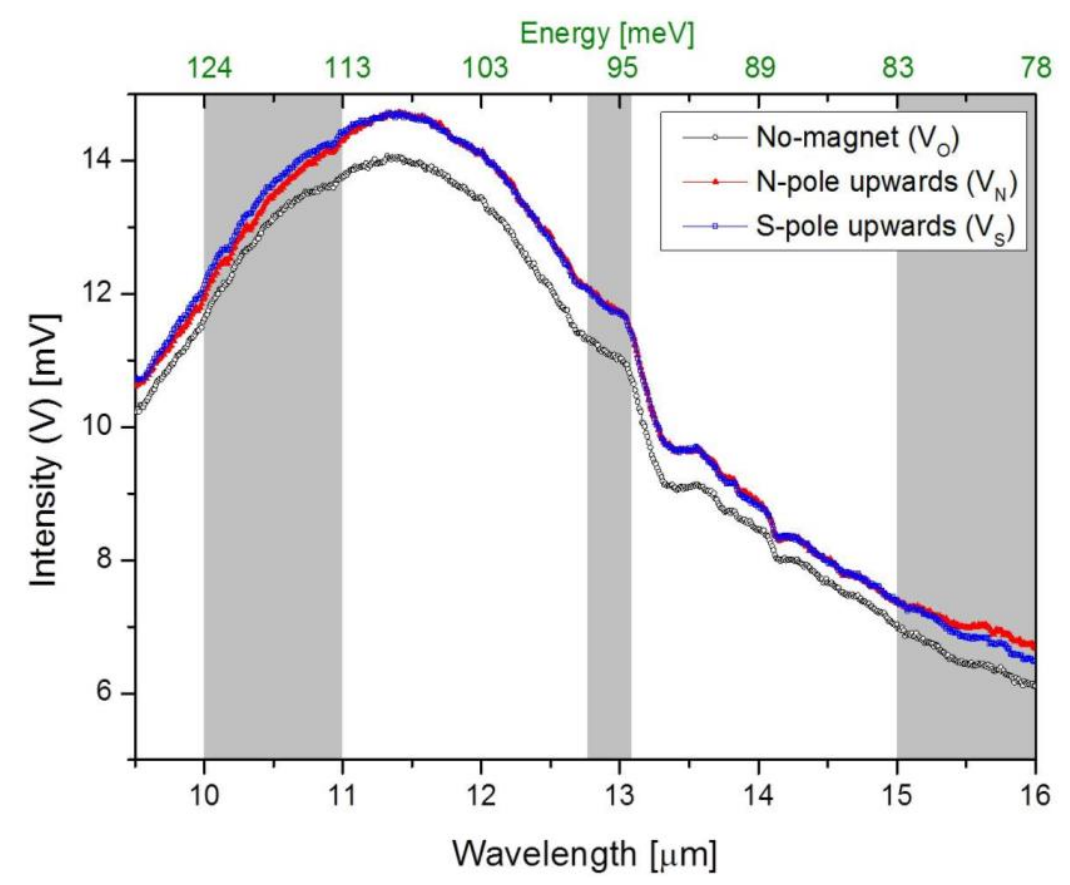

Fig. 5.6: Measured intensities for the as-deposited $\mathrm{Co}_{2} \mathrm{FeSi} / \mathrm{Si}$ films without and with the fields with $\mathrm{N}$ and S-pole upwards in the wavelength range between 9.5 and $16 \mu \mathrm{m}$. 


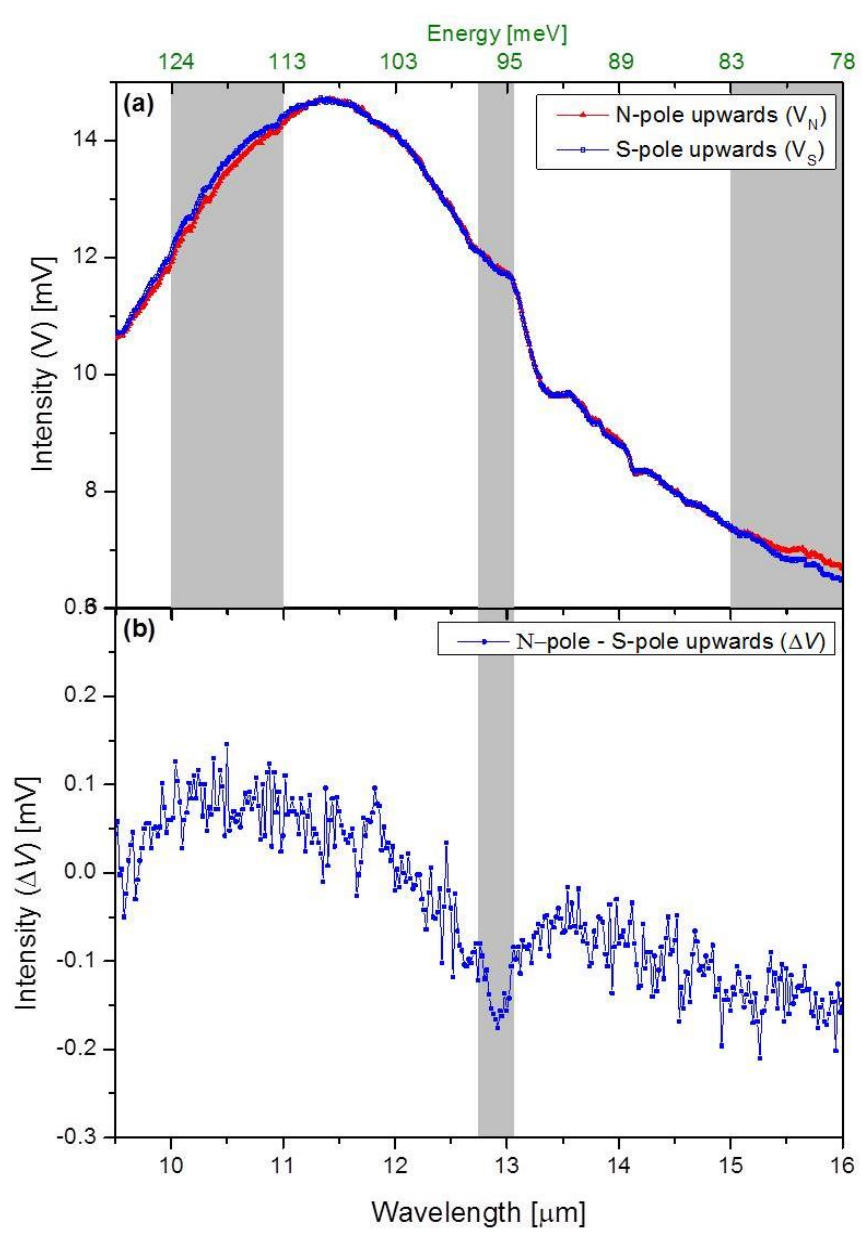

Fig. 5.7: Intensities for the as-deposited $\mathrm{Co}_{2} \mathrm{FeSi} / \mathrm{Si}$ films (a) with the fields with the $\mathrm{N}$ - and S-pole upwards and (b) their differences in the wavelength range between 9.5 and $16 \mu \mathrm{m}$.

The CFS films were then annealed at $400^{\circ} \mathrm{C}$ for up to $3 \mathrm{~h}$ in $1 \mathrm{~h}$ steps (see Fig. 5.8). The differences $\Delta V$ for the annealed films did not show a clear dip corresponding to a band-gap, which suggested that the films might have improved their crystalline ordering as expected but they might have formed several phases, hindering the presence of their band-gaps. This might agree with the crystalline ordering found in the polycrystalline films with the $L 2_{1}$ or $B 2$-ordered grains and disordered matrices as shown in Fig. 5.2(a). These preliminary results confirm the validity of this band-gap measurement technique. In the next section, higher ordered films will be used for the measurement to determine the band-gap. 


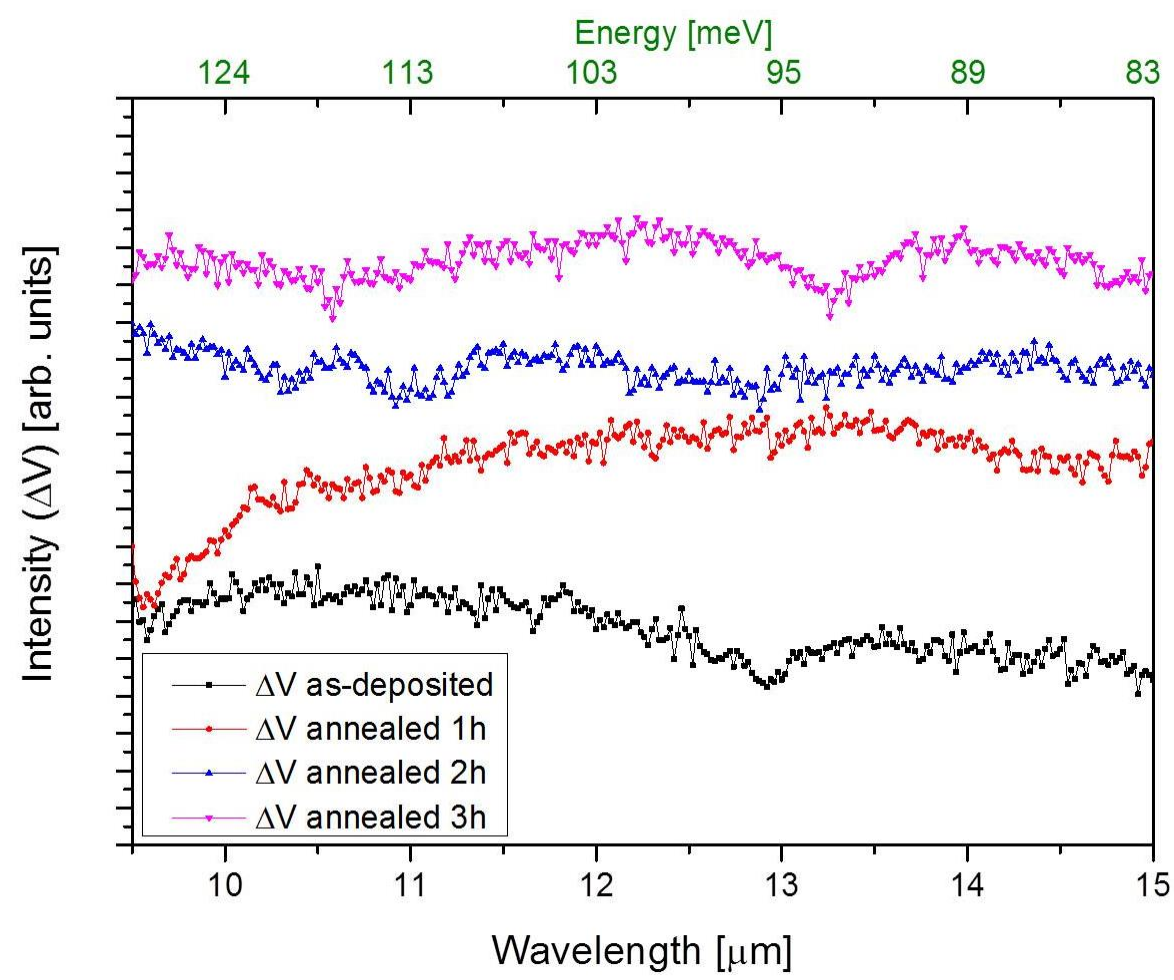

Fig. 5.8: Intensity differences $\left(\Delta V=V_{\mathrm{N}}-V_{\mathrm{S}}\right)$ for the $\mathrm{Co}_{2} \mathrm{FeSi} / \mathrm{Si}$ films between the magnetic fields with the N- and S-pole upwards as-deposited and after post-annealing at $400^{\circ} \mathrm{C}$ for up to 3 hours.

\subsection{2. $\mathrm{Co}_{2} \mathrm{FeSi} / \mathrm{MgO}(001)$}

In addition to the CFS/Si films, another band-gap measurements were conducted on CFS films that grown on $\mathrm{MgO}(001)$ substrates. The lattice constant of the $\mathrm{MgO}(001)$ is $0.4212 \mathrm{~nm}[147]$ and the lattice mismatch with the CFS is 35\% [144]. However, these films showed better crystalline ordering as discussed below. The intensity signals were also measured for the as-deposited CFS films grown on $\mathrm{MgO}(001)$ substrates without applying a magnetic field and with the fields with the $\mathrm{N}$ - and S-pole upwards as shown in Fig. 5.9. The three signals were similar as for the $\mathrm{Co}_{2} \mathrm{FeSi} / \mathrm{Si}$ films but with a minor difference at the wavelength of $\sim 13 \mu \mathrm{m}$. These films were anticipated to form better crystalline ordering as shown in Fig. 5.2(b). It should be noted that Figure 5.2(b) was taken on the UHV-sputtered film, which should have better ordering than the films studied here. 
Figure 5.10 shows a dip in the measured signals only for one of the two field directions, i.e., the S-pole upwards. The dip becomes more prominent by subtracting the signals for the S-pole upwards from those for the N-pole upwards $(\Delta V)$. This clearly means only one of the spin bands absorbs the corresponding circularly polarised light. This proves the presence of the band-gap (94 meV) in the polycrystalline $\mathrm{Co}_{2} \mathrm{FeSi}$ film even in the as-deposited state. This dip is much more significant in the amplitude (200 $\mu \mathrm{V}$ ) as compared with that for the $\mathrm{Co}_{2} \mathrm{FeSi} / \mathrm{Si}$ films in Section 5.4.1. As the current measurement configuration uses a $45^{\circ}$ incident angle with the IR penetration depth of $20 \mu \mathrm{m}[70]$, it is sensitive to the bulk region of the film, confirming the presence of a half-metallic band gap at room temperature. This finding agrees with the previous report on the predominant $L 2_{1}$ ordering in polycrystalline $\mathrm{Co}_{2} \mathrm{FeSi}$ films grown on the $\operatorname{MgO}(001)$ substrates [143].

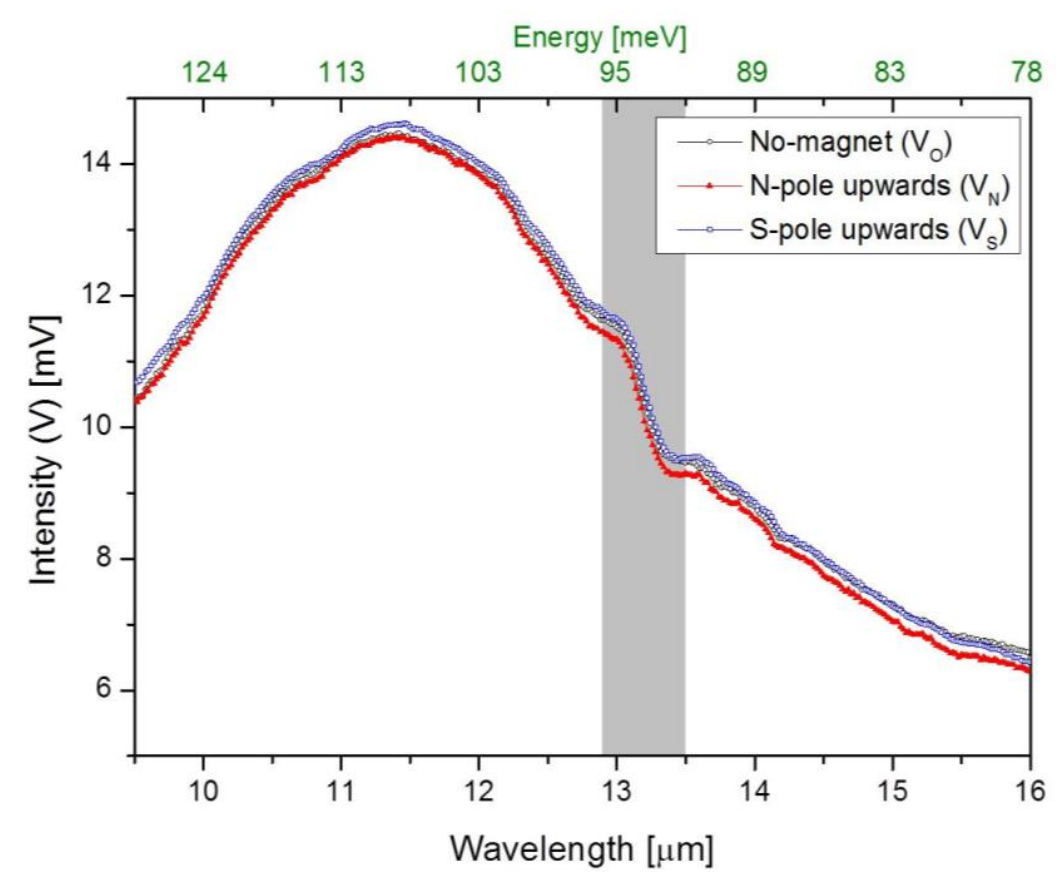

Fig. 5.9: Measured intensities for the as-deposited $\mathrm{Co}_{2} \mathrm{FeSi} / \mathrm{MgO}(001)$ films without and with the fields with the $\mathrm{N}$ - and S-pole upwards in the wavelength range between 9.5 and $16 \mu \mathrm{m}$. 


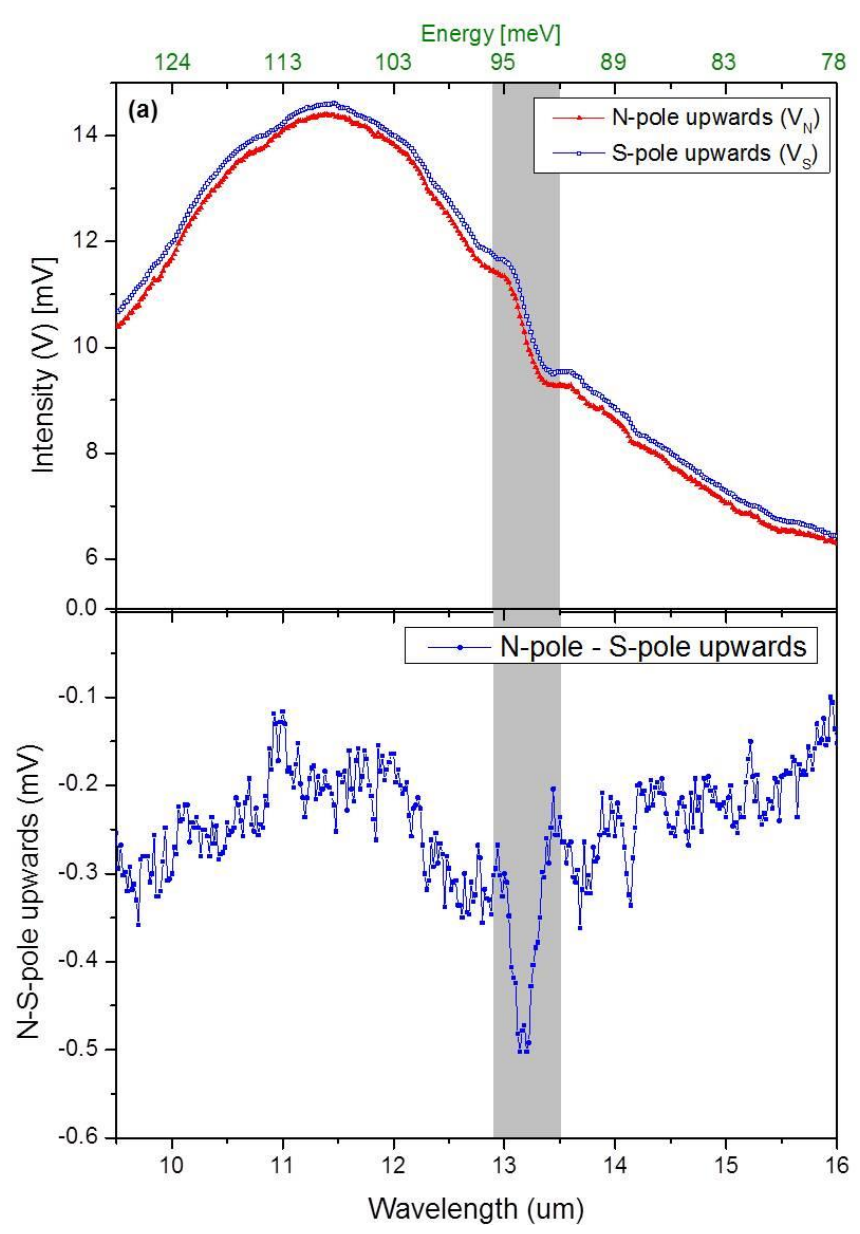

Fig. 5.10: Intensities for the as-deposited $\mathrm{Co}_{2} \mathrm{FeSi} / \mathrm{MgO}(001)$ films (a) with the fields with the $\mathrm{N}$ - and $\mathrm{S}$ pole upwards and (b) their differences in the wavelength range between 9.5 and $16 \mu \mathrm{m}$.

Figure 5.11 shows the intensity difference of the measurements under the magnetic fields with the $\mathrm{N}$ - and S-pole upwards in the wavelength range between 12 and $14 \mu \mathrm{m}$ for the as-deposited film (open square symbols) and after annealing for 3, 4.5 and 6 hours (open circle, solid triangle and solid circle symbols, respectively). The intensity curves for the as-deposited films show a dip corresponding to the $\mathrm{Co}_{2} \mathrm{FeSi}$ band-gap energy of $\sim 94 \mathrm{meV}$ as discussed above. This indicates the band-gap measured at RT is $64 \%$ of the value predicted (146 meV) using GGA calculations at $0 \mathrm{~K}$ [66]. The film was then measured after annealing at $400^{\circ} \mathrm{C}$ for 3 hours (open circles in Fig. 5.11) which was expected to possess the improved crystalline ordering as detected by the $\mathrm{XRD}$ as shown in Fig. 5.1. The magnitude of the peak was increased to $\sim 300 \mathrm{mV}$ at the 
same wavelength, i.e., $13.2 \mu \mathrm{m}$, indicating that the crystalline ordering of the sample was improved and the volume of the ordered phase increased to absorb more IR light for photoexcitation across the band-gap.

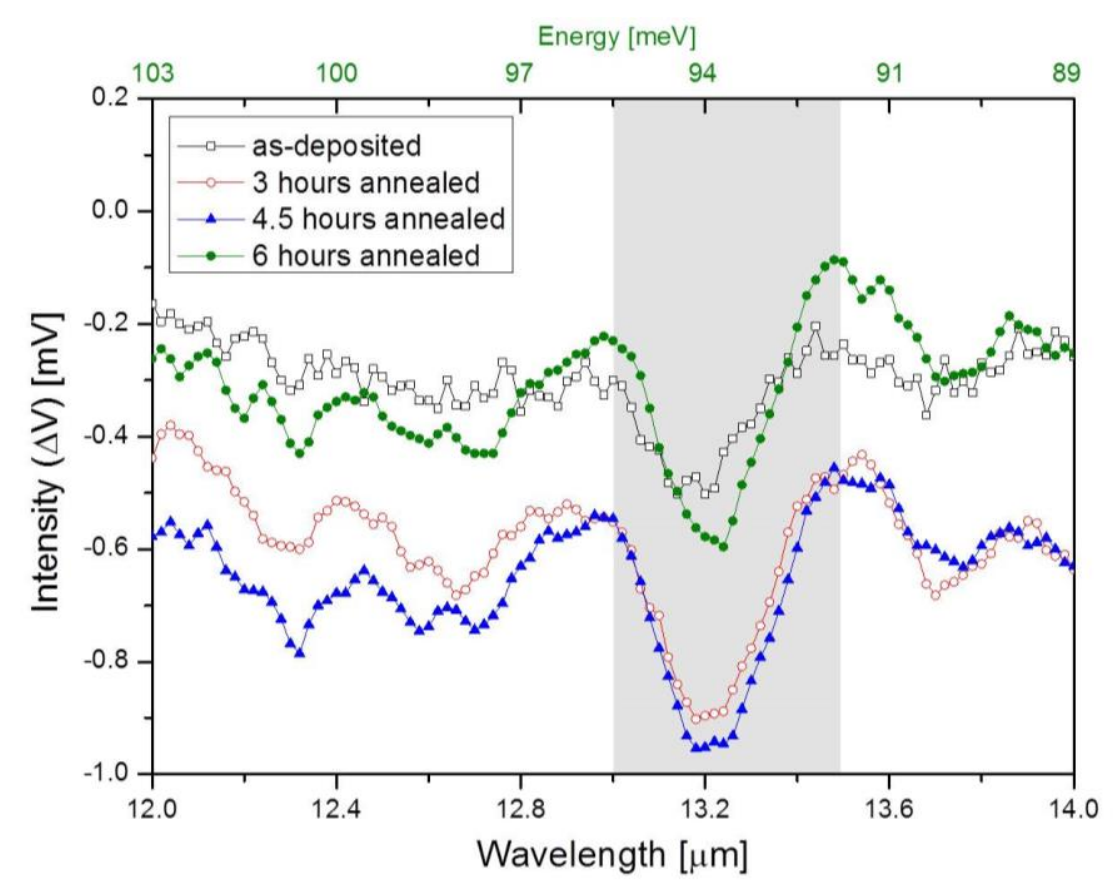

Fig. 5.11: Intensity differences between the magnetic fields with the $\mathrm{N}$ - and S-pole upwards $\left(\Delta V=V_{\mathrm{N}}-\right.$ $V_{\mathrm{S}}$ ) for $\mathrm{Co}_{2} \mathrm{FeSi} / \mathrm{MgO}(001)$ films as-deposited and after post-annealing at $400^{\circ} \mathrm{C}$ for up to 6 hours.

The film was also measured after annealing at $400^{\circ} \mathrm{C}$ for an additional 1.5 hours for a total of 4.5 hours. The peak magnitude was further increased to $\sim 350 \mathrm{mV}$ at the same wavelength as can be seen in Fig. 5.11 (solid triangles), again indicating that the crystallinity of the sample was improved further. The film was further measured after annealing for another 1.5 hours for a total of 6 hours. However, the peak magnitude remained at $\sim 350 \mathrm{mV}$ which suggested that the crystallinity of the film had reached its saturation. The $\Delta V$ magnitude was hence found to increase with longer annealing time up to 4.5 hours. This tendency agrees with the XRD results in Fig. 5.1 as well as the previous studies on crystalline ordering [138].

Figure 5.12 summarises a clear increase in the dip amplitude in the measured difference between the two field directions with increasing annealing time up to $4.5 \mathrm{~h}$ at 
$400^{\circ} \mathrm{C}$. This indicates that the crystalline ordering of the $\mathrm{Co}_{2} \mathrm{FeSi}$ films improves with increasing annealing time as discussed above. This also agrees with the increase in $M_{\mathrm{s}}$ measured. After 4.5 hours annealing, $M_{\mathrm{S}}$ is measured to be $\sim 760 \mathrm{emu} / \mathrm{cm}^{3}$, which is $\sim$ $62 \%$ of the theoretically predicted value [68] and is similar to the previously reported value on a polycrystalline CFS film $\sim 800 \mathrm{emu} / \mathrm{cm}^{3}$ ) [137]. The annealing for $6 \mathrm{~h}$ induces the Si segregation as reported [137], reducing $M_{\mathrm{s}}$ significantly $\left(\sim 460 \mathrm{emu} / \mathrm{cm}^{3}\right)$. This agreement also confirms that the measured band-gap represent the magnetic properties of the films.

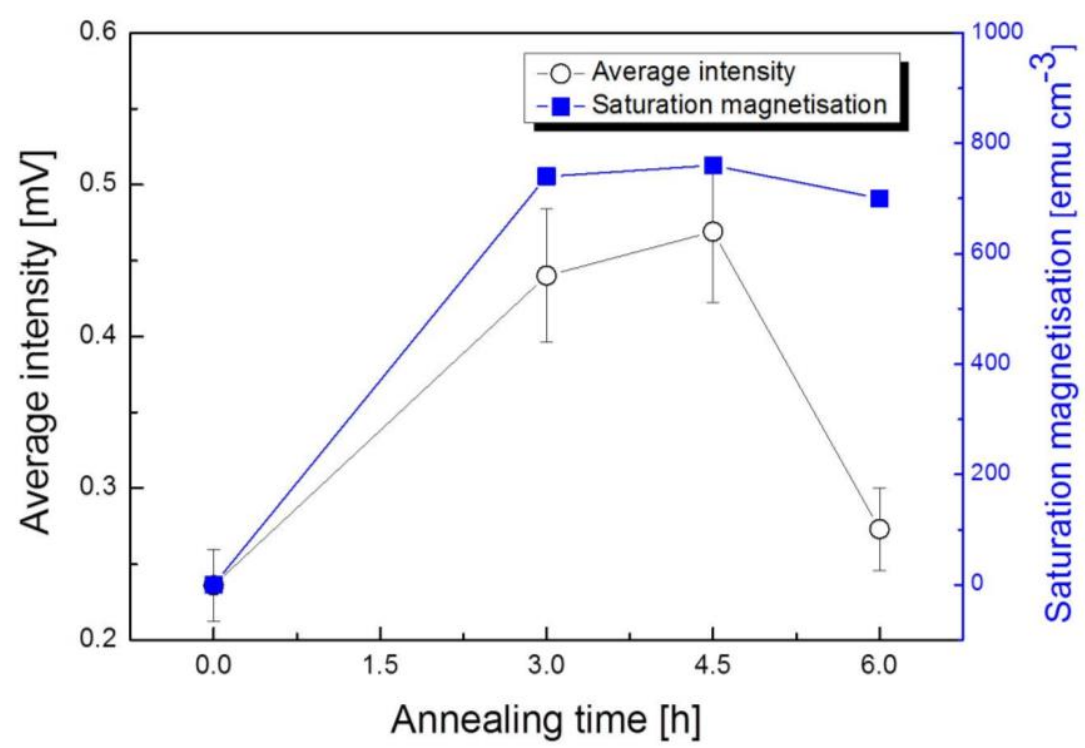

Fig. 5.12: Annealing time dependence of the intensity difference in the intensity between the fields with the N- and S-pole upwards. The corresponding saturation magnetisation is also plotted. 


\subsection{Summary}

In this chapter, band-gap measurements on polycrystalline $\mathrm{Co}_{2} \mathrm{FeSi}$ films grown on $\mathrm{Si} / \mathrm{SiO}_{2}$ and $\mathrm{MgO}(001)$ substrates are discussed. The band-gap measurements are conducted by introducing circularly polarised IR light $(h v)$ with the energy ranging between 78 and $413 \mathrm{meV}$, which is expected to cover the range of the band-gap $\left(E_{\mathrm{g}}\right)$ of the CFS films. The CFS films grown on $\mathrm{Si} / \mathrm{SiO}_{2}$ substrates show a band-gap estimation of (96 meV) only for the as-deposited films, which can be attributed to the broad distributions in their crystalline ordering after post-annealing. On the other hand, the $\mathrm{CFS} / \mathrm{MgO}(001)$ films show better and more consistent results with the band-gap of 94 $\mathrm{meV}$ for the as-deposited and annealed films at $400^{\circ} \mathrm{C}$ for up to 6 hours. The increase in the measured intensity agrees with the improvement of the crystalline ordering and the resulting increase in the saturation magnetisation of the films. These properties take their maxima at 4.5 hour annealing due to the Si segregation by further annealing.

These results prove that our technique allows for the measurement of the halfmetallic band-gap directly at room temperature. By reducing the IR incident angles from the film plane, our technique enables the measurement of the band-gaps of the surface regions (i.e., few nanometres) . This may provide direct feedback for the growth optimisation in order to realise a larger magnetoresistance in a half-metallic Heusleralloy junction at room temperature. 


\section{Chapter 6}

\section{Heusler Band-Gap Measurements on}

\section{Epitaxial $\mathrm{Co}_{2} \mathrm{FeAl}_{0.5} \mathrm{Si}_{0.5} / \mathrm{MgO}(001)$}

\subsection{Introduction}

$\mathrm{Co}_{2} \mathrm{FeAl}_{0.5} \mathrm{Si}_{0.5}$ CFAS full-Heusler alloy is one of the most studied Heusler alloys [70] owing to its high $T_{\mathrm{C}}$ and high $P . T_{\mathrm{C}}$ of CFAS has been reported to be $\sim 1100 \mathrm{~K}$ [26][27], which is similar to $T_{\mathrm{C}}$ value of CFS as discussed in Chapter 5. $P$ of CFAS has been estimated using Julliere's formula in MTJs to be as high as 91\% at RT [28][75], although it has also been reported to be slightly smaller to be between 56 and $70 \%$ at RT by the other groups [71][72]. At low temperature, $P$ has been estimated in MTJs to be $81 \%$ at $5 \mathrm{~K}[73]$ and $71 \%$ at $7 \mathrm{~K}$ [74], while it reaches only $\sim 60 \%$ at $5 \mathrm{~K}$ using a PCAR technique [76]. Unlike CFS whose $E_{\mathrm{F}}$ lies near the conduction band [148], $E_{\mathrm{F}}$ of CFAS is near the centre of the minority gap [28][77]. Therefore, CFAS exhibits the smallest temperature dependence of its spin polarisation [28][77] and it is expected to improve the temperature dependence of the corresponding tunnelling magnetoresistance (TMR) ratio [73]. This has been demonstrated by Shan et al. [28] with a CFAS/ $\left(\mathrm{MgAl}_{2}\right) \mathrm{O}_{\mathrm{x}} / \mathrm{CoFe} \mathrm{MTJ}$, where the TMR ratio is $160 \%$ at $7 \mathrm{~K}$ and is reduced 
almost linearly to $100 \%$ at RT. Accordingly, a MTJ using CFAS electrodes and a MgO barrier has been fabricated, showing the largest TMR ratio of 386\% at RT [149]. Interestingly, it is found that CFAS follows the generalised Slater-Pauling curve and its $m$ has been experimentally reported to be $\sim 5.5 \mu_{B} /$ f.u. [77][150], which is consistent with calculations [77].

CFAS has been fabricated in a film form by sputtering [74][151] and MBE deposition [79] and in a bulk form by arc melting [76][77]. Tables 6.1 and 6.2 below summarises the magnetic properties of CFAS. The $E_{\mathrm{g}}$ of CFAS has been calculated to be $760 \mathrm{meV}$ using $\mathrm{LDA}+U$ [27][75-78], while it has been estimated to be $600 \mathrm{meV}$ in a MTJ at 7K [73].

Table 6.1: List of calculated magnetic properties of CFAS.

\begin{tabular}{|c|c|c|}
\hline $\begin{array}{c}\text { Magnetic moment }(\boldsymbol{m}) \text { in } \\
\text { formula unit }\left[\mu_{\mathrm{B}} / \mathrm{f} . \mathrm{u} .\right]\end{array}$ & $\begin{array}{c}\text { Band-gap }\left(\boldsymbol{E}_{\mathrm{g}}\right) \\
{[\mathrm{meV}]}\end{array}$ & $\begin{array}{c}\text { Curie Temperature }\left(\boldsymbol{T}_{\mathbf{C}}\right) \\
{[\mathrm{K}]}\end{array}$ \\
\hline $\begin{array}{c}5.5 \\
\text { (predicted by the Slater-Pauling } \\
\text { curve) [70] }\end{array}$ & $\begin{array}{c}760(\mathrm{LDA}+U) \\
{[70][75-78]}\end{array}$ & $1150 \pm 50[27]$ \\
\hline $5.5[71]$ & & \\
\hline
\end{tabular}


Table 6.2: List of magnetic properties of CFAS obtained experimentally.

\begin{tabular}{|c|c|c|c|c|}
\hline $\begin{array}{c}\text { Magnetic moment } \\
(m) \text { in formula unit } \\
{\left[\mu_{B} / \text { f.u. }\right]}\end{array}$ & $\begin{array}{c}\text { Saturation } \\
\text { magnetisation } \\
(M S)\left[\mathrm{emu} / \mathrm{cm}^{3}\right] / \\
\mu_{B} / \text { f.u. }\end{array}$ & $\begin{array}{c}\text { Spin } \\
\text { polarisation }(P)\end{array}$ & $\begin{array}{c}\text { Band-gap } \\
\left(\boldsymbol{E}_{\mathrm{g}}\right) \\
{[\mathrm{meV}]}\end{array}$ & $\begin{array}{c}\text { Curie } \\
\text { Temperature } \\
\left(T_{\mathrm{C}}\right) \\
{[\mathrm{K}]}\end{array}$ \\
\hline $\begin{array}{c}5.98 \text { at } 5 \mathrm{~K} \text { (bulk) } \\
{[71]}\end{array}$ & $5.61 \mu_{\mathrm{B}} /$ f.u. $[70]$ & $\begin{array}{c}91 \% \text { (MTJ) at } \\
\text { RT [28] }\end{array}$ & $\begin{array}{c}600 \text { (MTJ) at } \\
7 \mathrm{~K}[73]\end{array}$ & $\begin{array}{c}1100 \text { (bulk) } \\
\text { [26] }\end{array}$ \\
\hline $\begin{array}{c}5.29 \text { at } 10 \mathrm{~K} \text { (bulk) } \\
{[71]}\end{array}$ & $\begin{array}{c}5.33 \pm 0.135 \mu_{\mathrm{B}} / \text { f.u. } \\
{[75]}\end{array}$ & $\begin{array}{c}70 \%(\mathrm{MTJ}) \text { at } \\
\text { RT ann. at } 400^{\circ} \mathrm{C} \\
{[72]}\end{array}$ & $\begin{array}{c}1200 \text { (bias } \\
\text { voltage } \\
\text { dependence of } \\
d I / d V \text { ) at } 7 \mathrm{~K} \\
{[73]}\end{array}$ & \\
\hline \multirow[t]{6}{*}{$\begin{array}{c}\sim 5.5 \text { at } 5 \mathrm{~K} \text { (bulk) } \\
{[77]}\end{array}$} & $880 \mathrm{emu} / \mathrm{cm}^{3}[80]$ & $\begin{array}{l}56 \% \text { (MTJ) at } \\
\text { RT, ann. at } \\
600^{\circ} \mathrm{C}[72]\end{array}$ & & \\
\hline & $900 \mathrm{emu} / \mathrm{cm}^{3}$ [74] & $\begin{array}{c}60 \pm 1 \% \text { (PCAR) } \\
\text { at } 5 \mathrm{~K} \text { (bulk) [76] }\end{array}$ & & \\
\hline & $\sim 900 \mathrm{emu} / \mathrm{cm}^{3}[79]$ & $\begin{array}{c}\text { 68\% (MTJ) at } \\
\text { RT [76] }\end{array}$ & & \\
\hline & $1100 \mathrm{emu} / \mathrm{cm}^{3}[81]$ & $\begin{array}{c}71 \%(\mathrm{MTJ}) \text { at } 7 \mathrm{~K} \\
{[74]}\end{array}$ & & \\
\hline & $1100 \mathrm{emu} / \mathrm{cm}^{3}[82]$ & $\begin{array}{c}81 \% \text { (MTJ) at } 5 \mathrm{~K} \\
{[73]}\end{array}$ & & \\
\hline & & $\begin{array}{c}81 \% \text { (MTJ) at } \\
\text { RT [149] }\end{array}$ & & \\
\hline
\end{tabular}




\subsection{Film growth}

Epitaxial CFAS thin films were grown on $\mathrm{MgO}(001)$ substrates by UHV-MBE (Eiko, $H D H-10000)$. The $\mathrm{MgO}(001)$ substrates were pre-cleaned by acetone, IPA and deionised water in ultrasonic bath for $5 \mathrm{~min}$. each. The substrate was then mounted on the sample holder and was cleaned with Nitrogen gas blow before loaded into the MBE chamber. The base pressure of the MBE system was $1.2 \times 10^{-11} \mathrm{~Pa}$ and the pressure during the deposition was typically $1.5 \times 10^{-10} \mathrm{~Pa}$. The films were deposited at a rate of $0.001 \mathrm{~nm} / \mathrm{s}$ at RT. The lateral size and thickness of the CFAS films were $5 \times 5 \mathrm{~mm}^{2}$ and $23 \mathrm{~nm}$, respectively. A $2 \mathrm{~nm}$ Au capping layer was deposited on CFAS to prevent oxidation. The films were then annealed ex situ at $400^{\circ} \mathrm{C}$ for up to 6 hours with intervals of 1.5 hours. During annealing, the films were mounted in a quartz glass tube inside a furnace (Carbolite, MTF10/28 A) with Nitrogen gas flowing at a rate of $100 \mathrm{sccm}$ with the corresponding pressure of $\sim 2.7 \mathrm{kPa}$. After the annealing, the films were kept inside the furnace to cool down to RT for $\sim 4$ hours.

Both the structural and magnetic characterisations were carried out for these films in their as-deposited and annealed states. The crystallographical analysis was performed by XRD (Rigaku, SmartLab) and TEM (JEOL, JEM-ARM200). The magnetic measurements were done using a VSM (Microsense, Model 10). The details of these methods were described in Section 6.3.

\subsection{Film characterisation}

In order to confirm the crystallisation of CFAS into the $L 2_{1}$ or $B 2$ full-Heusler phase, the films have been characterised using XRD. Figure 6.1(a) shows XRD intensity measured by a conventional $\theta-2 \theta$ out-of-plane thin-film set up. Two principal $\mathrm{MgO}$ peaks are visible at around $43^{\circ}$ and $94^{\circ}$ together with secondary radiation peaks from $\mathrm{W}$ 
and the $\mathrm{K}$ beta $\mathrm{X}$-rays at $38^{\circ}, 41^{\circ}, 82^{\circ}$ and $88^{\circ}$. There is a peak at $22^{\circ}$ which might be for the capping layer (i.e., Au). The peak at $66^{\circ}$ is related to the CFAS(400) peak, which has been reported in Ref. 3. The presence of the CFAS(400) and (220) peaks is an indicator of the formation of the $B 2$ structure. There are also some peaks underneath the large $\mathrm{MgO}$ peaks but it cannot be identified without using a monochromator in the XRD, which is broken recently.

Therefore, $2 \theta_{\mathrm{b}}$ scan was conducted to identify these peaks precisely. This was performed by attempting to observe the CFAS(220) peak, which should be present at $2 \theta_{\mathrm{b}}=44.8^{\circ}$. The orientation of CFAS was found to be (001) in the plane of the film as expected. Therefore to detect the (220) plane the gun/detector geometry was arranged at $45^{\circ}$ to that of the plane of the film. This was further beneficial to avoid the overlap between the CFAS(220) peak and the shoulder of the $\mathrm{MgO}(001)$ peak at $\sim 44^{\circ}$. The $2 \theta_{\mathrm{b}}$ results can be seen in Fig. 6.1(b) for the samples as-deposited and after 6 hours of annealing at $400^{\circ} \mathrm{C}$, which shows a clear peak at $2 \theta_{\mathrm{b}}=44.8^{\circ}$. The higher end of the $2 \theta_{\mathrm{b}}$ peak cannot be measured due to the limitations of the goniometer movement. By fitting these results, the full widths half maximum (FWHM) of the peaks are estimated to be $1^{\circ}$ and $0.8^{\circ}$ for the as-deposited and 6-hour annealed films, respectively. This shows that the crystallinity of the film is almost independent of annealing but it does improve slightly by annealing.

The $2 \theta_{\chi}$ in-plane scan [see Fig. 6.2(a)] again reveals the presence of the CFAS(220) peak at the predicted at an angle of $45^{\circ}$, which cannot be seen clearly in the above scans. This scan has been conducted by adjusting the detector at a very low angle of $0.4^{\circ}$. However, this scan suffered from noisy signals due to the low-angle setting. 

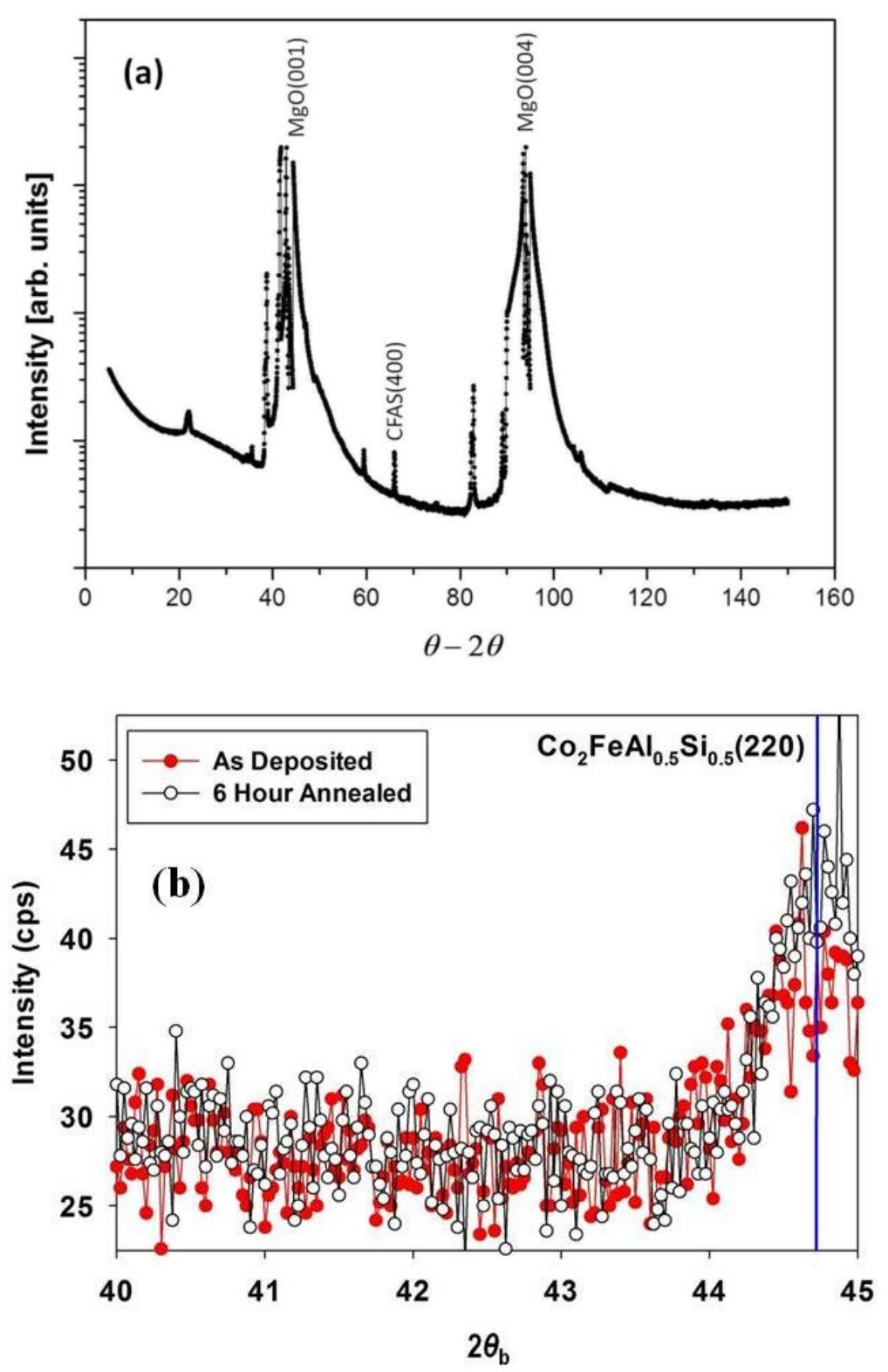

Fig. 6.1: (a) XRD intensities of the 6-hour annealed CFAS/MgO(001) films using a $\theta-2 \theta$ set up and (b) the corresponding XRD intensities of the as-deposited (closed circles) and after 6-hour annealed (open circles) $\mathrm{CFAS} / \mathrm{MgO}(001)$ films using a $2 \theta_{\mathrm{b}}$ set up. 

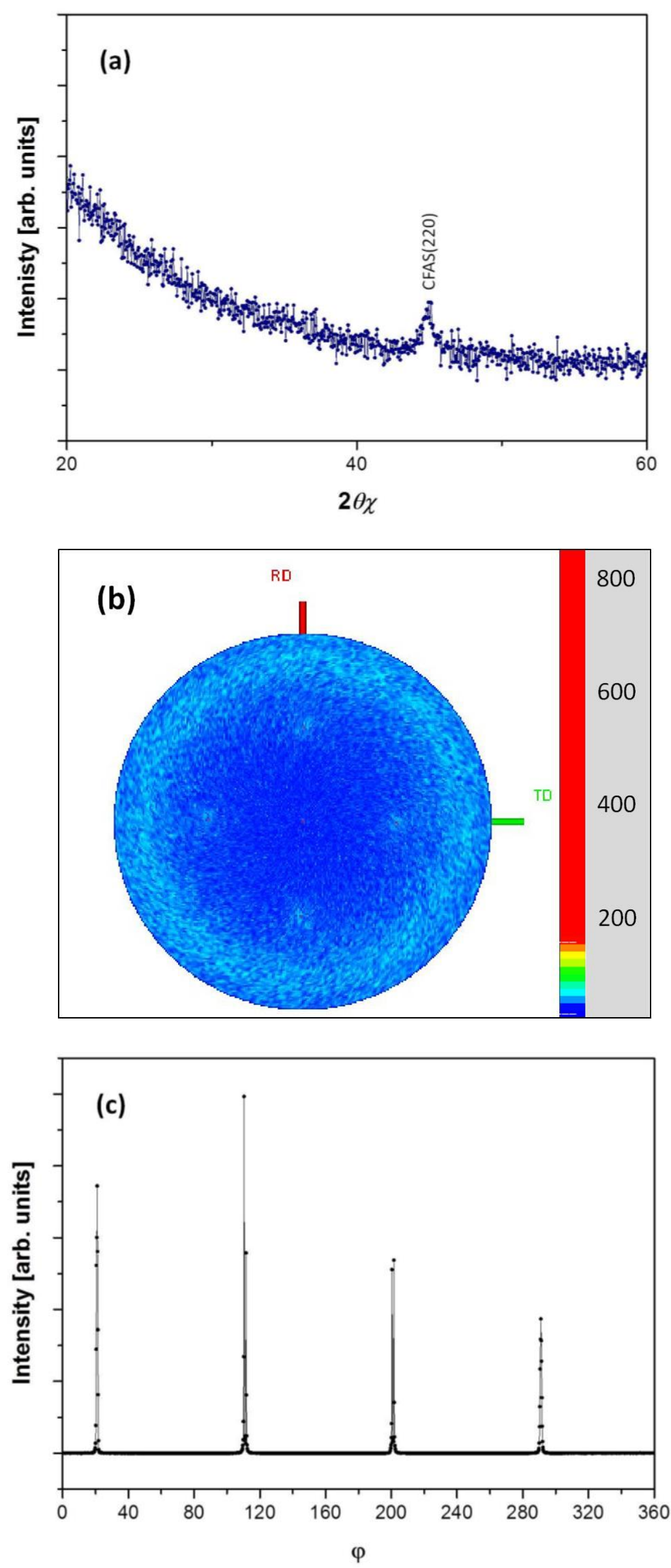

Fig. 6.2: XRD analysis of the 6-hour annealed films using (a) $2 \theta \chi$ (b) pole figure and (c) the rotation $\varphi$ scan setups are also shown. 

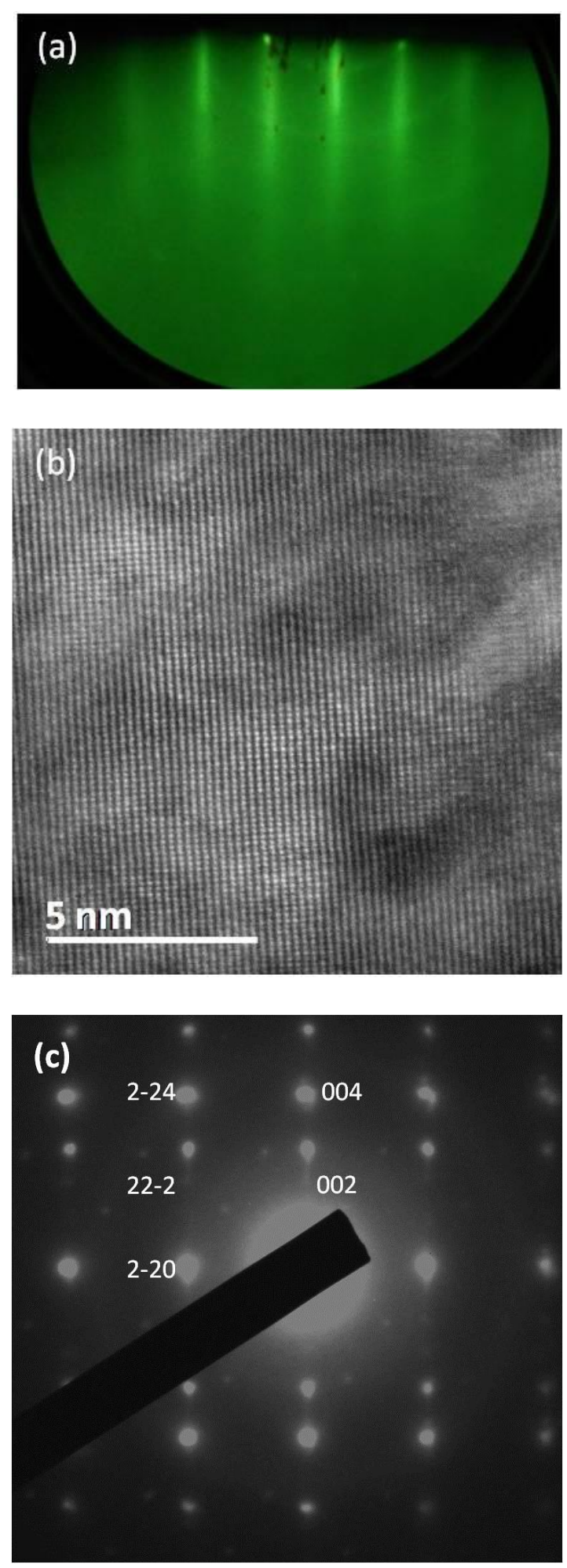

Fig. 6.3: (a) In-situ reflection high-energy electron diffraction (RHEED) pattern with the electron beam introduced along $\mathrm{MgO}(100)$, (b) cross-sectional TEM image and (c) the corresponding selected area electron diffraction (SAED) pattern of the as-deposited (001) CFAS films.

Figure 6.2(b) shows a pole figure pattern around the CFAS(400) peak. It shows two weak sets of four-fold symmetric reflections. The outer ring, which corresponds to 
the substrate, shows much stronger intensity than the film's intensity (in the inner ring). The rotational $\varphi$ scan [see Fig. 6.2(c)] confirms the presence of the cubic Heusler structure due to the four-fold symmetry indicated by the spacing of $90^{\circ}$ between the spots. This is an indication of almost perfect crystalline growth in the in-plane direction as the spot sizes are very small (FWHM: $0.77^{\circ}$ ). These spots correspond to CFAS (400) and hence indicate that the film might have a mixture of both $B 2$ and $L 2_{1}$ phases. It should be noted that the low signal counts from the sample is due to the small sample size (i.e., $5 \times 5 \mathrm{~mm}^{2}$ ) where it is preferred to a have sample size of 1 " $\times 1$ " or at least 1 $\times 1 \mathrm{~cm}^{2}$ to obtain more XRD counts.

In order to confirm the crystalline ordering, Figure 6.3 shows RHEED and crosssectional TEM observations images taken on the as-deposited CFAS film. These images clearly demonstrate the epitaxial growth of the CFAS films. Cross-sectional TEM specimen preparation has been done by mechanical polishing followed by argon ion polishing in order to achieve electron transparency [82]. The lattice constant extracted from the TEM image is $0.5546 \mathrm{~nm}$, which agrees very well with the bulk value reported $(0.5685 \mathrm{~nm})$ [76]. The SAED image confirms the presence of the $B 2$ phase in the CFAS films even in the as-deposited state. These results prove the high quality of the films with the $B 2$ phase (and possibly the $L 2_{1}$ phase after annealing).

Figure 6.4 shows the magnetisation curves of the CFAS films with magnetic fields applied perpendicular and parallel to the films. $M_{\mathrm{s}}$ for the as-deposited and 6-hour annealed films are measured to be $(1007$ and $1237 \pm 10 \%) \mathrm{emu} / \mathrm{cm}^{3}$, which are 92 and $112 \%$ of the theoretically predicted value $\left(1100 \mathrm{emu} / \mathrm{cm}^{3}\right)$, respectively [139]. These values again confirm the minor improvement is achieved by the ex situ annealing. Such enhancement in $M_{\mathrm{s}}$ over prediction has been reported under the coexistence of the $L 2_{1^{-}}$ordered phase with minor disordered and/or segregated phases [80][139]. The coexisting phases may also be responsible for the minor step in the magnetisation loop 
as appeared for the as-deposited film in Fig. 6.4(a).

The coercivity $\left(H_{\mathrm{c}}\right)$ is also measured to be 305 and 30 Oe for the out-of- and inplane loops for the as-deposited film, respectively. Here $H_{\mathrm{c}}$ is similar to those measured for the polycrystalline as-deposited films as reported previously, i.e., 480 and 38 Oe for the out-of- and in-plane configurations, respectively [152]. $H_{\mathrm{c}}$ increases slightly to be 309 and 26 Oe for the out-of- and in-plane loops for the 6-hour annealed film, respectively. Since the permanent magnet used for the band-gap measurements generates a field of $2.2 \mathrm{kOe}$, the films should be fully saturated during the measurements. These loops have the squareness of 0.76 and 0.77 under a field applied in-plane and perpendicular to the films, respectively. These values are similar to the previous reports [121]. These results therefore confirm the epitaxial films have a high degree of ordering and are ideal for the band-gap measurements in the next section.

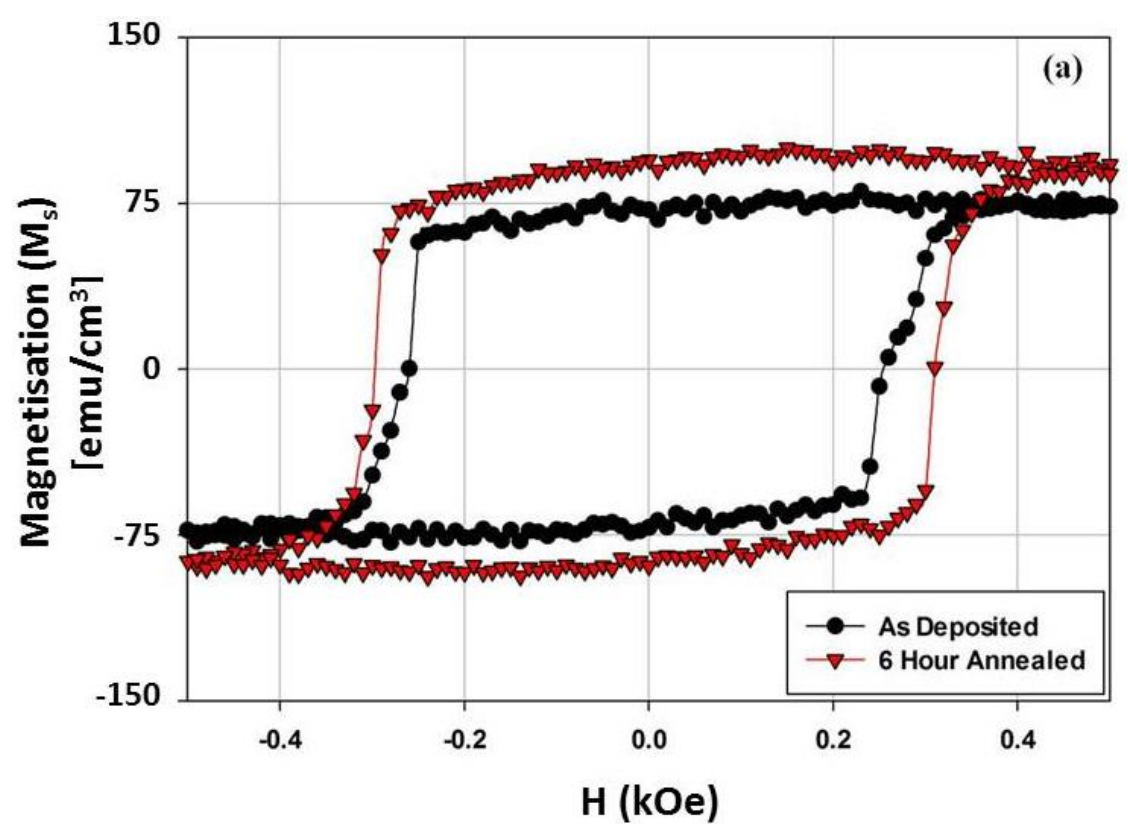




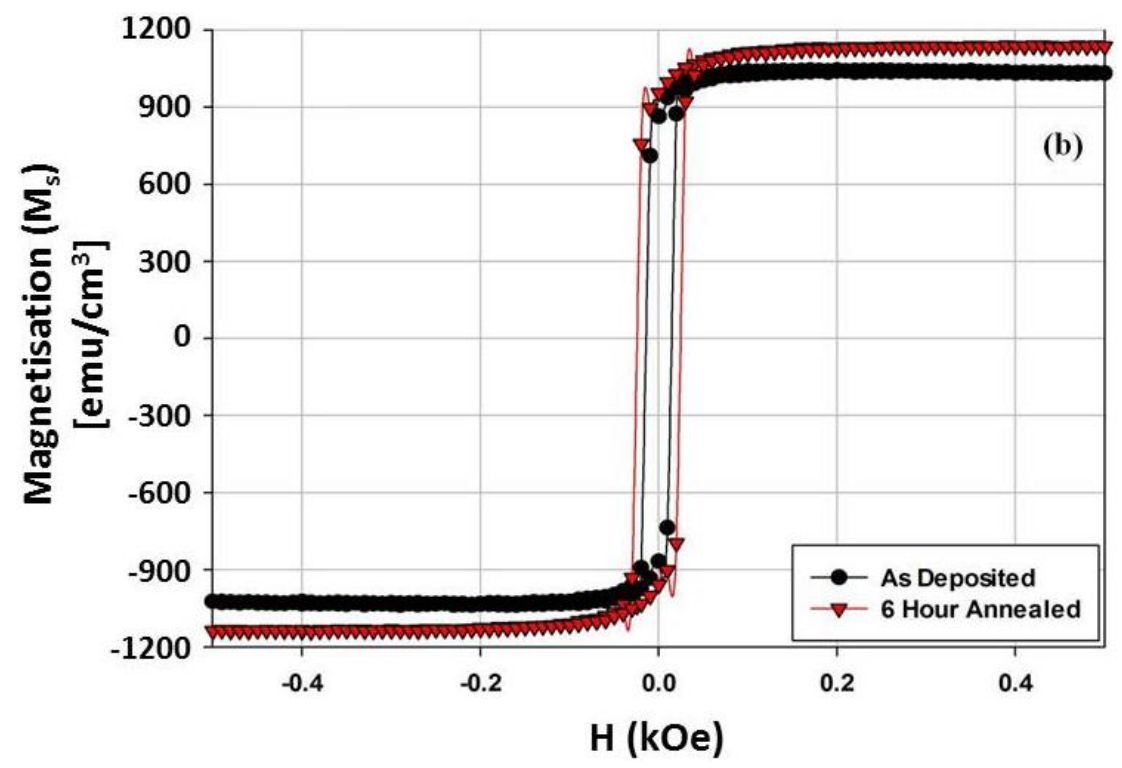

Fig. 6.4: Hysteresis loops of the as-deposited (closed circles) and 6-hour annealed (closed triangles) CFAS/MgO(001) films under magnetic fields applied (a) perpendicular and (b) parallel to the film plane.

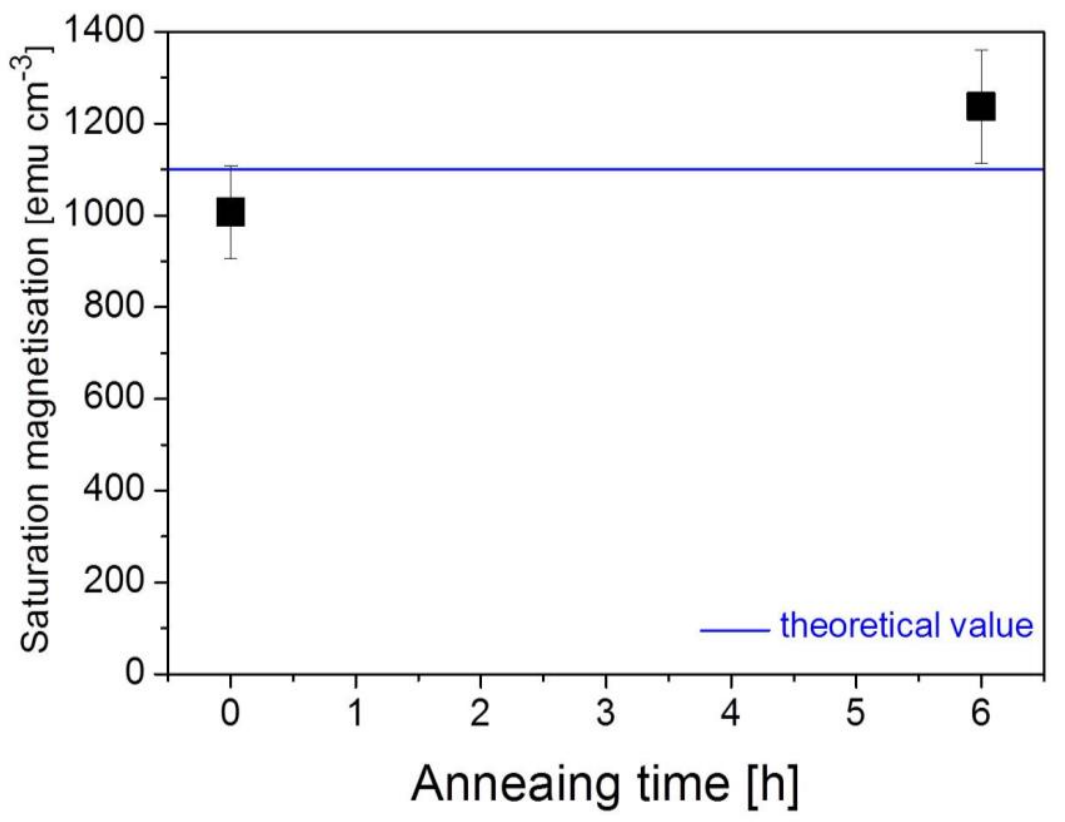

Fig. 6.5: Annealing time dependence of saturation magnetisation of the epitaxial $\mathrm{CFAS} / \mathrm{MgO}(001)$ films. 


\subsection{Band-gap measurements}

The intensity signals were measured for the as-deposited CFAS films grown on $\mathrm{MgO}(001)$ substrates without applying a magnetic field and with the fields with the $\mathrm{N}$ and S-pole upwards as shown in Fig. 6.5. The three intensity signals were similar with minor differences at the wavelengths ranging between 10.5 and $13.6 \mu \mathrm{m}$ (as shaded in grey in Fig. 6.6). The detailed differences between the intensity signals that measured with the fields with the N- and S-pole upwards were evaluated as shown in Fig. 6.7(a). A clear dip was observed in the difference signal $\left(\Delta V=V_{\mathrm{N}}-V_{\mathrm{S}}\right)$ as shown in Fig. 6.7(b). $\Delta V$ revealed the dip at the wavelength of $\sim 11.2 \mu \mathrm{m}$ with the amplitude of $\sim$ $0.132 \mathrm{mV}$. This dip was expected to be related to the band-gap of $\sim 111 \mathrm{meV}$ for the CFAS films epitaxially grown on $\mathrm{MgO}(001)$ substrates.

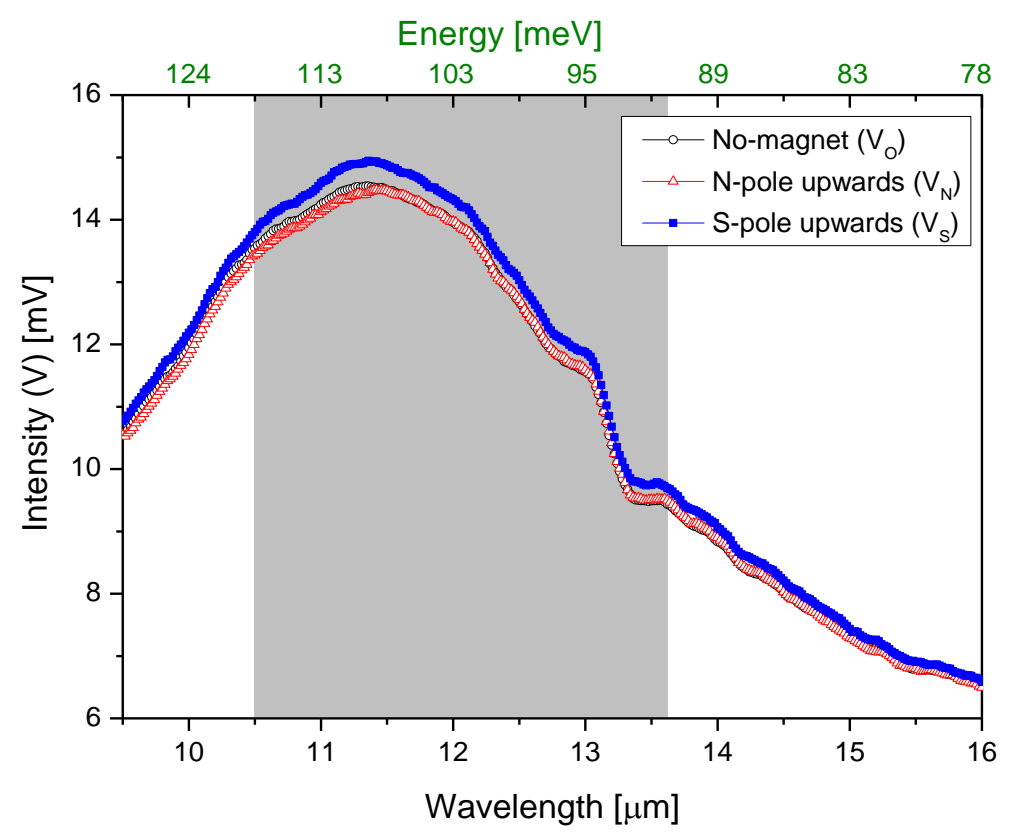

Fig. 6.6: Measured intensities for the as-deposited $\mathrm{Co}_{2} \mathrm{FeAl}_{0.5} \mathrm{Si}_{0.5} / \mathrm{MgO}(001)$ films without and with the fields with $\mathrm{N}$ - and S-pole upwards in the wavelength range between 9.5 and $16 \mu \mathrm{m}$. 


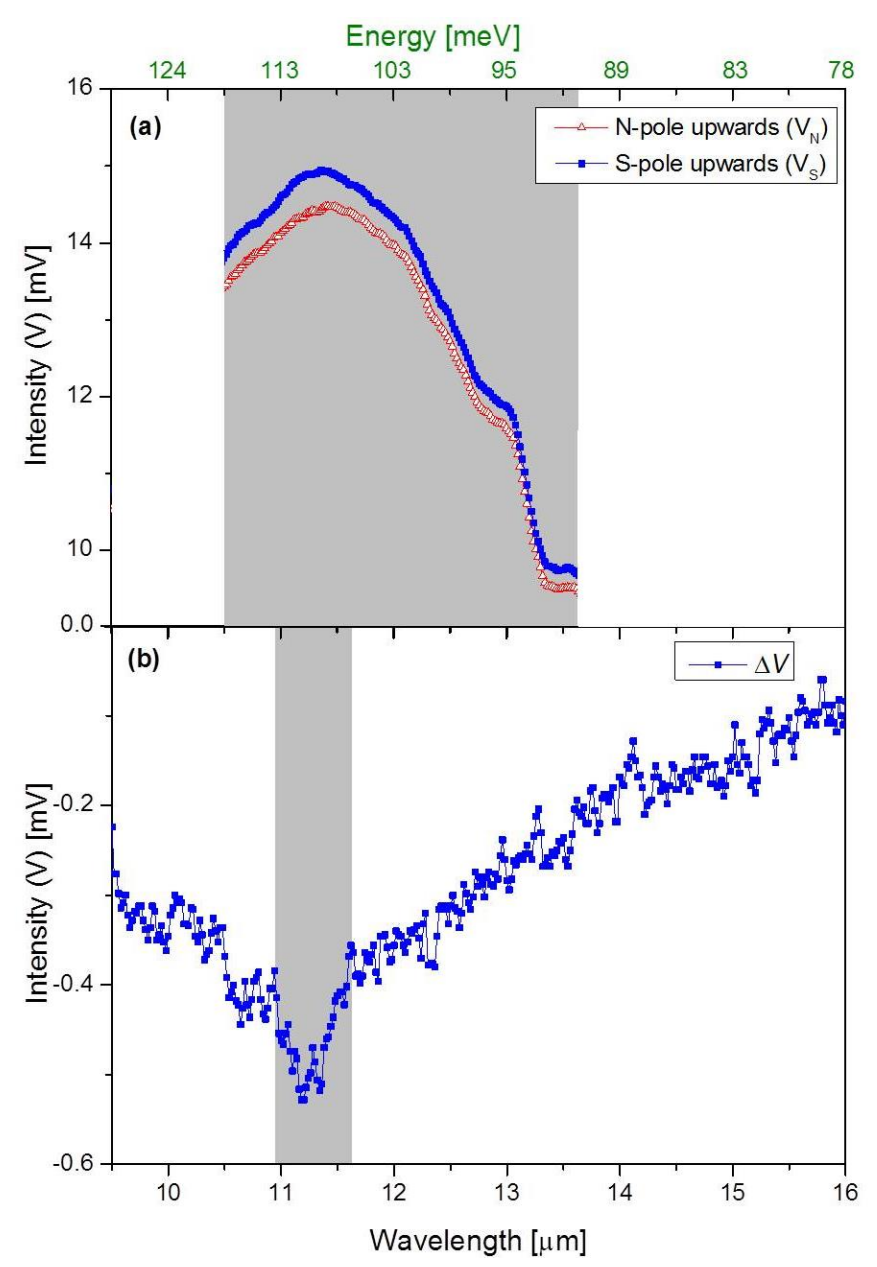

Fig. 6.7: Intensities for the as-deposited $\mathrm{Co}_{2} \mathrm{FeAl}_{0.5} \mathrm{Si}_{0.5} / \mathrm{MgO}(001)$ films (a) with the fields with the $\mathrm{N}-$ and S-pole upwards and (b) their differences in the wavelength range between 9.5 and $16 \mu \mathrm{m}$.

Figure 6.8 shows the intensity differences measured under the magnetic fields with the $\mathrm{N}$ - and S-pole upwards for the as-deposited film (open square symbols) and for the annealed films for 1.5, 3, 4.5 and 6 hours (open triangle, open circle, solid triangle and solid circle symbols, respectively) in the wavelength ranging between 9.5 and 16 $\mu \mathrm{m}$. For the as-deposited films, a dip is obtained at (11.2 \pm 0.3$) \mu \mathrm{m}$ with the amplitude of $(0.132 \pm 0.012) \mathrm{mV}$ which is due to the absorption of the IR radiation at the band-gap as discussed in Section 3.1.1. This corresponds to the band-gap of $(111 \pm 2) \mathrm{meV}$. In previous studies, the band-gap of epitaxial CFAS has been estimated to be $1.2 \mathrm{eV}$ from the bias voltage dependence of differential tunnelling conductance $G=d I / d V$ at $7 \mathrm{~K}$ [73], while the band-gap has been measured in the same study to be half of the 
calculation value $(600 \mathrm{meV})$ using the Julliere model in a MTJ at low temperature $(7 \mathrm{~K})$. The reason for this discrepancy between the two values may be the lower ordering parameter at the interfaces of the CFAS film [73]. The magnetic tunnelling spectroscopy in an epitaxial $\mathrm{Co}_{2} \mathrm{MnSi}$ tunnel junction has also been used to measure the band-gap at RT, resulting $30 \mathrm{meV}$ [153]. The latter value is much smaller than our measurement, which is likely to be due to the interface sensitive measurements using MTJ. Our measured band-gap is in a good agreement with the former reported values by considering the band-edge broadening with increasing temperature.

After annealing the films at $400^{\circ} \mathrm{C}$ for 1.5 and 3 hours, dips are similarly observed at an IR wavelength of $(13.1 \pm 0.2) \mu \mathrm{m}$. This indicates a minor reduction in the band-gap to $(95 \pm 1) \mathrm{meV}$. The amplitudes of the peaks are $(0.12 \pm 0.005)$ and $(0.096 \pm 0.004) \mathrm{mV}$ for the 1.5 and 3 hour annealed films, respectively. These amplitudes are slightly smaller as compared with those of the as-deposited film. It can be noted that these films show wider dips (i.e., $0.53-0.9 \mu \mathrm{m}$, compared to $\sim 0.5 \mu \mathrm{m}$ for the CFS films) near the initial band-gap as shaded in Fig. 6.8. These findings may indicate that the epitaxial films have several phases with different band-gaps, which is different from the polycrystalline cases as previously discussed in Sections 5.4.1 and 5.4.2. This may be occurred by the strain relaxation during the annealing, which is initially induced by the lattice mismatch between CFAS (lattice constant, $a=0.5685 \mathrm{~nm}$ ) [70] and $\mathrm{MgO}$ (lattice constant, $a=0.4212 \mathrm{~nm})$ aligned with $45^{\circ}$ rotation $(0.4212 \sqrt{2}=0.5957 \mathrm{~nm})$ [70]. The $45^{\circ}$ rotation reduces the lattice mismatch to be $\sim 4.6 \%$ as reported [70]. The lattice mismatch is the difference between lattice constants of both the substrate and the film in which smaller difference provides epitaxial growth with loss defects. 


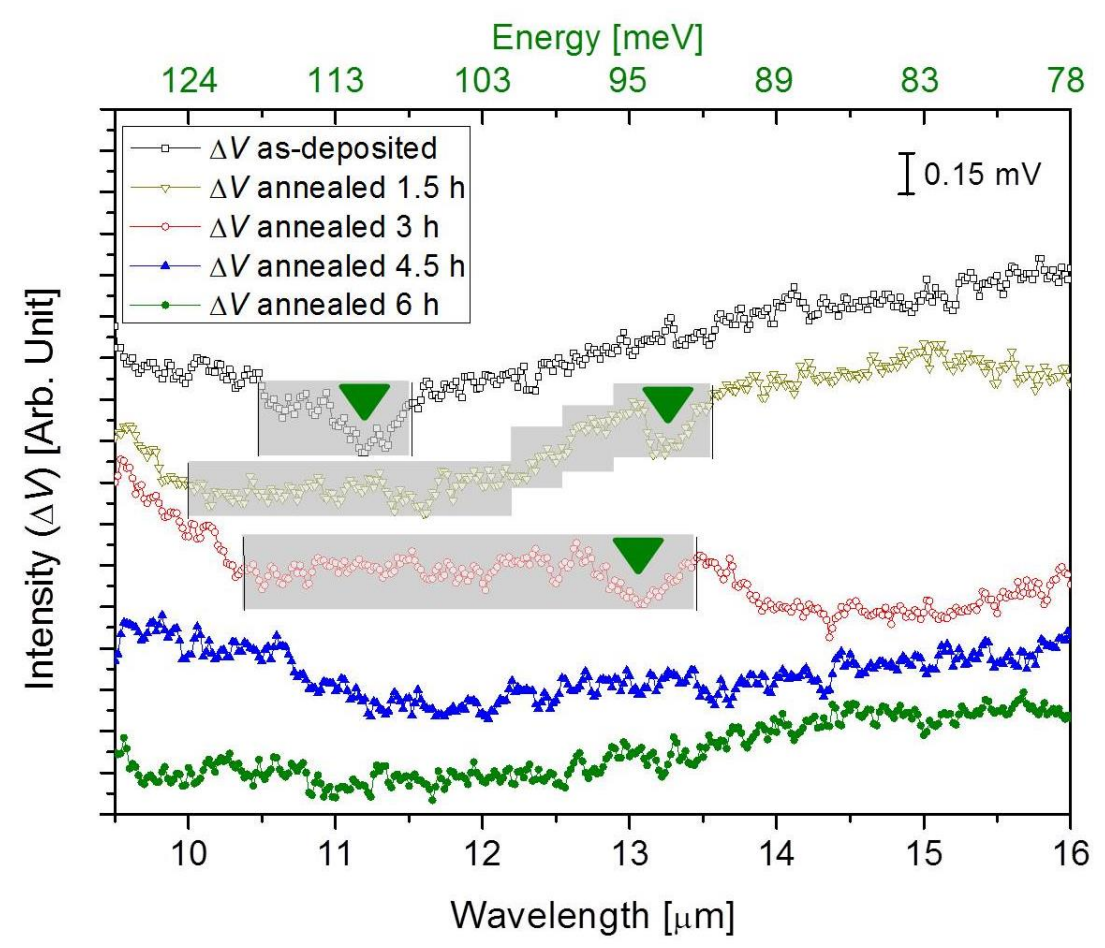

Fig. 6.8: Differences in measured intensities $\left(\Delta V=V_{\mathrm{N}}-V_{\mathrm{S}}\right)$ under the magnetic fields with the $\mathrm{N}$ - and $\mathrm{S}$ pole upwards to CFAS/MgO(001) films after post-annealing at $400^{\circ} \mathrm{C}$ for up to 6 hours. Green arrows represent the band-gap energy peak and grey shades show regions with minor changes in signals.

Sagar et al. [154] have reported the formation of periodic strain relaxing regions at the CFS/MgO interface with the period of $(5.4 \pm 0.1) \mathrm{nm}$ as shown in Fig. 6.9. These regions may be responsible for the different phases in the CFAS films, which is similar to CFS. This indicates that the polycrystalline Heusler alloy films are advantageous over the epitaxial films unless the latter films are free from interfacial strain by inserting a lattice-matching buffer layer between the substrate and the film. 


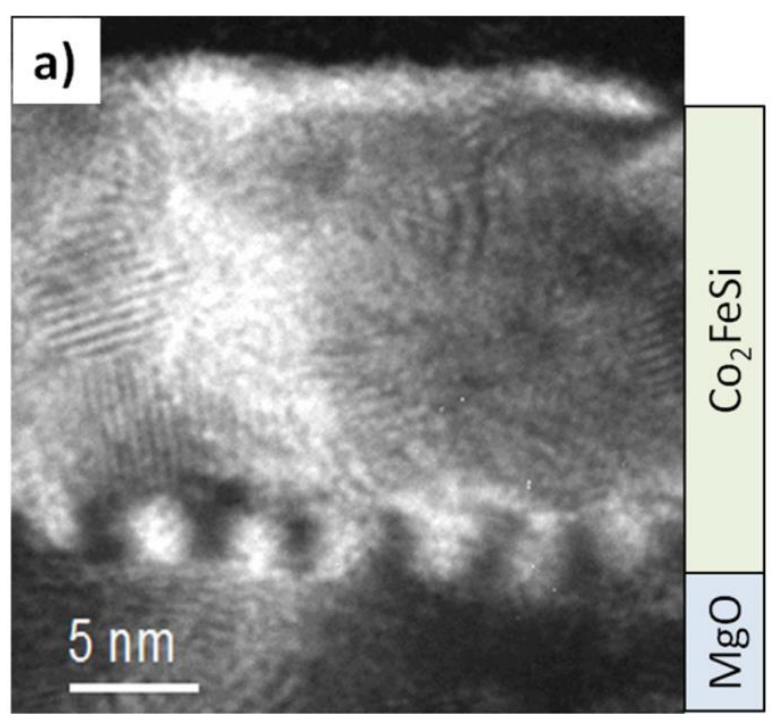

Fig. 6.9: Weak-beam dark-field image of $\mathrm{Co}_{2} \mathrm{FeSi} / \mathrm{MgO}(001)$ interface showing dislocation cores at the interface. The figure taken from [154].

Finally the band-gap is vanished after annealing over $4.5 \mathrm{~h}$, which agrees with our previous report on the polycrystalline films due to $\mathrm{Si}$ segregation [152]. This tendency is summarised in Fig. 6.10. Figure 6.10 also agrees with the annealing time dependence of the corresponding saturation magnetisation as shown in Fig. 6.5.

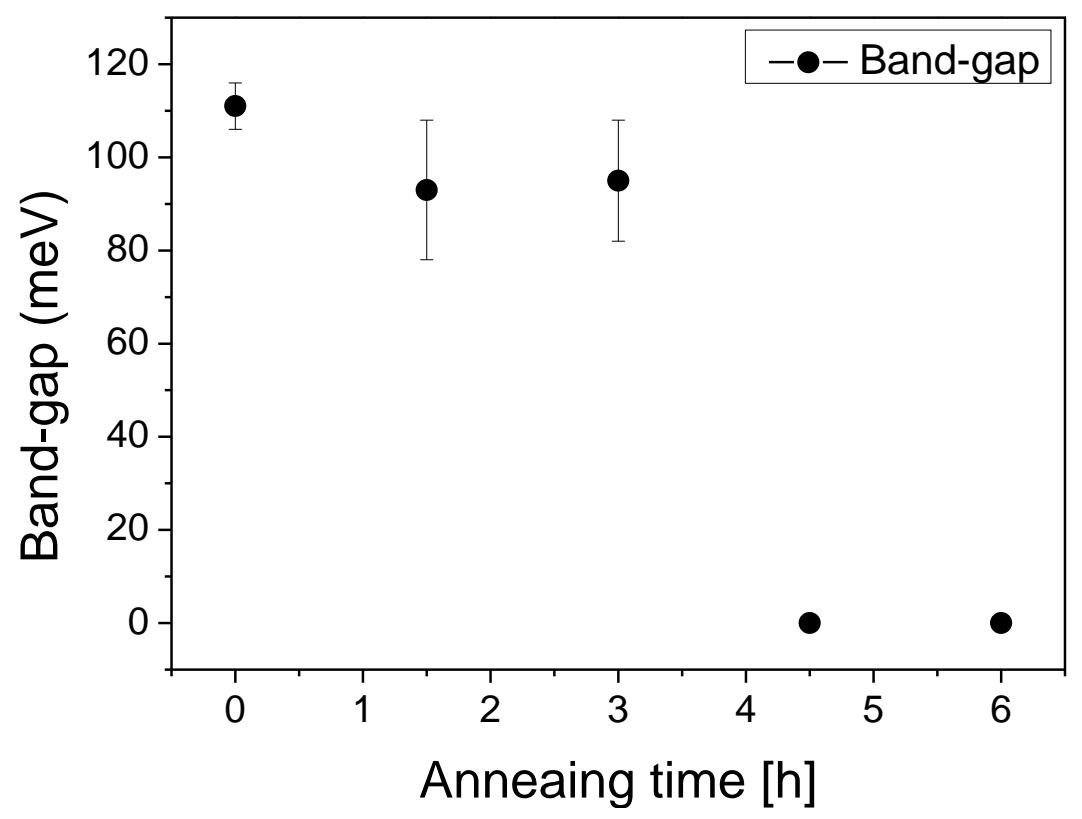

Fig. 6.10: Annealing time dependence of estimated band-gap energies of the epitaxial CFAS/MgO(001) films. 


\subsection{Summary}

Band-gap measurements on epitaxial CFAS films grown on $\mathrm{MgO}(001)$ substrates were conducted using the developed technique. Similar to the measurements on the polycrystalline CFS films, the band-gap of the CFAS films were measured for both the as-deposited and post-annealed films at $400^{\circ} \mathrm{C}$ for up to 6 hours with intervals of 1.5 hours. The band-gap of the as-deposited CFAS films was estimated to be $\sim 111 \mathrm{meV}$. It was also found that the band-gap was shifted slightly to be $\sim 95 \mathrm{meV}$ for the postannealed CFAS films for up to 3 hours, which suggested the presence of different phases formed after annealing. The band-gap was vanished after annealing over $4.5 \mathrm{~h}$, which agreed with the previous report on polycrystalline CFS films due to phase segregation in Chapter 5.

These results again prove that this technique allows for the measurement of the half-metallic band-gap directly at room temperature. It can be noted that the band-gaps of CFAS has smaller amplitudes (i.e., the intensity difference between the top and bottom of the dip corresponding to the band gap) as compared to these of the CFS films. However, this technique can resolve signals as small as a $\mu \mathrm{V}$ order. 


\section{Chapter 7}

\section{Heusler Band-Gap Measurements on a}

\section{NiMnSb Heusler Alloy}

\subsection{Introduction}

$\mathrm{NiMnSb}$ is a half-Heusler alloy and is the first compound that has been theoretically predicted to be a HMF [83][155]. Based on electronic band structure calculations at $0 \mathrm{~K}$, the bulk NMS has been predicted to possess a band-gap in the minority spins of $\sim 0.5$ $\mathrm{eV}$ and consequently exhibit $100 \%$ spin polarisation at the Fermi level [4][71]. It crystallises in a cubic structure $C 1_{b}$ [84][85]. Its $T_{\mathrm{C}}$ is measured and calculated to be in the range of $728-1112 \mathrm{~K}$ [71][86][87]. NMS therefore becomes attractive as a spin source in spintronic devices [84][85][156] due to its large band-gap [4][24][71][86] and its high $T_{C}$ of $>728 \mathrm{~K}[83-85][88][89]$.

Various techniques can be used to fabricate bulk and thin films of NMS. Arc melting [85][88][99] and rapid quenching [87] techniques have been used to fabricate bulk samples. MBE [84][155] and co-sputtering [71] have been employed to grow epitaxial films, while sputtering [83] and pulsed laser deposition (PLD) [24][88] have been employed to grow polycrystalline films. Table 7.1 summarises the magnetic 
properties of NMS.

The magnetic moment of NMS has been theoretically predicted to be $4 \mu_{B} /$ f.u. [24][85][86][88]. Different calculation methods have been applied to estimate the magnetic moments of NMS, leading to the values of $4 \mu_{B} /$ f.u. [157] and $3.94 \mu_{B} /$ f.u. [91] using first-principles and the layer Korringa-Kohn-Rostoker with the coherent potential approximation (LKKR-CPA) methods, respectively. A series of experiments on NMS bulk and thin films samples have been performed and the magnetic moment is found to be $\sim 4 \mu_{B}$ ff.u., which is in good agreement with the predicted value. For example, Gardelis et al. [85] and Ritchie et al. [86] have measured the magnetic moments of bulk NMS to be $(4 \pm 0.02)$ and $4.02 \mu_{B} /$ f.u., respectively. As listed in Table 7.1, the measured magnetic moments of NMS films show some variations between 3.5 and $4 \mu_{B} /$ f.u. [24]. The differences in the measured magnetic moments are due to the sample preparation [90] and resulting atomic disorder [85].

High spin polarisation of $99.3 \%$ and $99 \%$ has been predicted in NMS using DFT within LDA [97] and first-principles calculations [98], respectively. Spin-polarisation measurements on NMS also show good half-metallic behaviour. Spin-polarised positron-annihilation (SPPA) on bulk NMS at 8K [71][92][158] and spin-polarised inverse photoemission (SPIP) on epitaxial thin films [93] show 100\% spin polarisation at $E_{F}$. PCAR has been first used by Soulen et al. [7] to measure the spin polarisation of NMS, which has been estimated to be $(58 \pm 2.3) \%$. Using the same method, $P$ has been measured to be between 35 and $45 \%$ [85-87][94]. These values are smaller than the above values but these are in good agreement with $P$ of $50 \%$ obtained by photoemission technique [95]. Spin polarisation of 25 and $\sim 28 \%$ has also been estimated by MTJ with the NMS electrodes [155]. Spin-resolved photoemission (SRPES) technique has shown $P$ of up to $40 \%$ for NMS films [96]. The latter measurements show much lower values of $P$ than the expected $100 \%$. The discrepancy between the expected $100 \% P$ for bulk 
NMS and the lower measured values can be ascribed to a number of possible reasons, such as atomic disorder at the surface [85][156][159], the formation of minority surface states [156], surface terminations of $\mathrm{Ni}$ or MnSb [85][156], surface segregation [93], non-stoichiometric compositions [87] and the possible reduction in the band-gap to be below $0.5 \mathrm{eV}$ at the surface [93].

$E_{\mathrm{g}}$ of NMS has been theoretically predicted to be $\sim 0.5 \mathrm{eV}[13][24]$. It was also calculated using DFT and $E_{\mathrm{g}}$ was found to be $0.54 \mathrm{eV}$ [103]. However, much lower value has also been calculated by first-principles calculations to be only $0.168 \mathrm{eV}$ [100]. Experimental measurements of interband absorption using infrared have been conducted and $E_{\mathrm{g}}$ was estimated to be $\sim 0.4 \mathrm{eV}$ at RT [101]. It is therefore important to measure the NMS band-gap accurately at RT. 
Table 7.1: List of magnetic properties of the NiMnSb obtained experimentally and theoretically.

\begin{tabular}{|c|c|c|c|c|}
\hline $\begin{array}{c}\text { Magnetic } \\
\text { moment }(\boldsymbol{m}) \\
{\left[\mu_{B} / \text { f.u. }\right]}\end{array}$ & 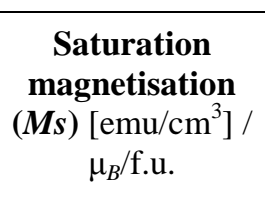 & $\begin{array}{c}\text { Spin polarisation } \\
(P)\end{array}$ & $\begin{array}{c}\text { Band-gap }\left(\boldsymbol{E}_{\mathrm{g}}\right) \\
{[\mathrm{meV}]}\end{array}$ & $\begin{array}{c}\text { Curie } \\
\text { Temperature } \\
\left(T_{\mathrm{C}}\right) \\
{[\mathrm{K}]}\end{array}$ \\
\hline $\begin{array}{c}4 \mu_{\mathrm{B}} / \mathrm{f.u.} . \\
\text { (bulk and film) } \\
\text { at } 5 \mathrm{~K}[88]\end{array}$ & $\begin{array}{c}3.6 \text { (film) } \\
\text { at } 4 \mathrm{~K}[84]\end{array}$ & $\begin{array}{c}50 \%-\text { bulk } \\
\text { (photoemission) } \\
{[95]} \\
\end{array}$ & $\begin{array}{c}\sim 500 \\
\text { (theoretically } \\
\text { predicted) [13] } \\
{[24][86][96][102]}\end{array}$ & $\begin{array}{c}730 \\
{[24][83-85]} \\
{[88-90][99]}\end{array}$ \\
\hline $3.9 \pm 0.2$ (film) [83] & $\begin{array}{c}4.0 \pm 0.06 \mu_{\mathrm{B}} / \text { f.u. } \\
\text { (film) } \\
\text { at } 5 \mathrm{~K}[89]\end{array}$ & $\begin{array}{c}35-50 \%-\text { bulk } \\
\text { (PCAR) } \\
\text { at } 1.5 \mathrm{~K}[87]\end{array}$ & $\begin{array}{c}168 \\
\text { (first-principles } \\
\text { calculations) [100] }\end{array}$ & $\begin{array}{c}728 \\
{[86][94]}\end{array}$ \\
\hline $\begin{array}{c}3.5 \mu_{\mathrm{B}} / \text { f.u. (film) } \\
\text { at } 5 \mathrm{~K} \text { [24] }\end{array}$ & $\begin{array}{c}\sim 538 \mathrm{emu} / \mathrm{cm}^{3} \\
\text { (film) } \\
\text { at } 5 \mathrm{~K} \text { [99] }\end{array}$ & $\begin{array}{c}\sim 44 \%-\text { bulk } \\
\text { (PCAR) } \\
\text { at } 4.2 \mathrm{~K}[94]\end{array}$ & $\begin{array}{c}540 \\
\text { (DFT calculation) } \\
{[103]}\end{array}$ & $\begin{array}{l}756 \\
{[92]}\end{array}$ \\
\hline $\begin{array}{c}3.6 \mu_{\mathrm{B}} / \text { f.u. (film) } \\
\text { at } 4 \mathrm{~K}[84]\end{array}$ & & $\begin{array}{l}\sim 45 \%-\text { bulk } \\
\quad \text { (PCAR) } \\
\text { at } 4.2 \mathrm{~K}[85][86]\end{array}$ & $\begin{array}{c}\sim 400 \\
\text { (measured by } \\
\text { infrared } \\
\text { absorption) at RT } \\
{[101]}\end{array}$ & \\
\hline $\begin{array}{c}3.94 \mu_{\mathrm{B}} / \text { f.u. } \\
\text { (calculation) [91] }\end{array}$ & & $\begin{array}{c}58 \pm 2.3 \% \text { (PCAR) } \\
{[7][71][95]}\end{array}$ & & \\
\hline $\begin{array}{c}4 \mu_{\mathrm{B}} / \text { f.u. (film) } \\
\text { at } 5 \mathrm{~K} \text { [24] }\end{array}$ & & $\begin{array}{c}20-40 \% \text { (Spin- } \\
\text { resolved } \\
\text { photoemission, } \\
\text { SRPES) - film } \\
{[96]}\end{array}$ & & \\
\hline $\begin{array}{c}4 \pm 0.02 \mu_{\mathrm{B}} / \text { f.u. } \\
\text { (bulk) at } 5 \mathrm{~K} \text { [85] }\end{array}$ & & $\begin{array}{l}28 \pm 2 \% \text { (spin- } \\
\text { polarised } \\
\text { tunneling } \\
\text { technique) - film } \\
\text { at } 0.4 \mathrm{~K}[155]\end{array}$ & & \\
\hline \multirow[t]{5}{*}{$\begin{array}{c}4.02 \mu_{\mathrm{B}} / \text { f.u. (bulk) } \\
\text { at } 5 \mathrm{~K}[86]\end{array}$} & & $\begin{array}{c}25 \% \text { (MTJ) at RT } \\
{[155]}\end{array}$ & & \\
\hline & & $\begin{array}{c}100 \%(100+0 / \\
-12 \%) \text { (spin- } \\
\text { polarized inverse } \\
\text { photoemission, } \\
\text { SPIP) - film at } \\
\text { 200K [93] }\end{array}$ & & \\
\hline & & $\begin{array}{c}99.3 \text { (Calculated- } \\
\text { DFT within LDA) } \\
{[97]}\end{array}$ & & \\
\hline & & $\begin{array}{c}99 \text { (Calculated- } \\
\text { first principle) } \\
{[98]}\end{array}$ & & \\
\hline & & $\begin{array}{l}\text { 100\% (Spin- } \\
\text { polarised positron- } \\
\text { annihilation, } \\
\text { SPPA) - bulk } \\
\text { at } 8 \mathrm{~K} \text { [158] }\end{array}$ & & \\
\hline
\end{tabular}




\subsection{Sample fabrication and characterisation}

Polycrystalline bulk samples of stoichiometric and non-stoichiometric NMS were fabricated in two stages at the University of Warwick. First stage is to determine the compositional ratios of NMS to produce stoichiometric single-phase and nonstoichiometric samples. Second stage is the production, where the samples were fabricated by arc melting method using a small tri-arc furnace. The samples were arc melted in an atmosphere of argon gas under pressure of $101.33 \times 10^{3} \mathrm{~Pa}$. Initial tests through several melting processes were performed to investigate the quantities of $\mathrm{Ni}$, Mn and Sb. During the initial tests, Sb was found to be lost in each melting process, which is required to add $3-5 \%$ more $\mathrm{Sb}$ than the stoichiometric ratio. The ratio of $\mathrm{Ni}: \mathrm{Sb}$ was found to be constant and does not require any corrections. However, the compositional ratio of Mn was found to be off-stoichiometric by Energy-dispersive Xray spectroscopy (EDX). By changing the amount of $\mathrm{Mn}$, a polycrystalline sample was produced, followed by post-annealing at $850^{\circ} \mathrm{C}$ for 150 hours. After annealing, a stoichiometric sample was fabricated. A purpose-built large four-arc furnace with constituent element ingots was used to produce single crystals. The NMS single-crystal boule was then sliced into small discs, followed by chemical-mechanical polishing to achieve mirror-like surfaces.

Crystalline structures of the NMS samples were characterised by XRD. $\theta-2 \theta$ scans were performed, showing the patterns with the expected NMS peaks and a small peak at $13^{\circ}$ related to the $\mathrm{Al}$ sample holder. The XRD patterns are similar to the theoretically predicted plots using the PowerCell software Package [160]. Therefore, the formation of the $C 1_{b}$ crystalline structure was confirmed and the lattice parameter was found to be $(0.5945 \pm 0.0001) \mathrm{nm}$ at RT [160]. Saturation magnetisation was measured to be $4.01 \mu_{\mathrm{B}} /$ f.u. [160], which is in agreement with the expected values. 


\subsection{Band-gap measurements}

The band-gap measurements were extended to the bulk NMS. Similar to the band-gap measurements for the CFS and CFAS thin films, the circularly polarised IR light was used, of which energy matched the expected $E_{\mathrm{g}}$ of NMS at RT. As expected, the bandgap of NMS was reduced at RT due to the reduction in the saturation magnetisation at finite temperatures [87].

\subsubsection{Stoichiometric sample}

The IR light was introduced to the surface of the NMS samples at $45^{\circ}$. The reflected intensity signal from the sample was measured for the entire wavelength range between 3 and $16 \mu \mathrm{m}$ without applying a magnetic field. The intensity signals were then measured with applying magnetic fields with the $\mathrm{N}$ - and S-pole upwards to the sample. The magnet used introduced a field of $\sim 2.2 \mathrm{kOe}$, which was sufficient to magnetise the sample as $H_{\mathrm{c}}$ was known to be very small $(<1$ Oe for bulk NMS and $\sim 2$ Oe for film NMS) [88][89][99]. The measurements under magnetic fields did not show clear evidence of the band-gap. Hence, the intensity difference between the $\mathrm{N}$ - and S-pole upwards was calculated. There was no significant feature in the intensity difference (blue line) in Fig. 7.1 except a dip at $\lambda \sim 7 \mu \mathrm{m}$ (corresponding to energy band-gap of $\sim$ $0.175 \mathrm{eV}$ ). A similar dip was also obtained only with applying a field with the N-pole upwards (black line). This was a key criterion as discussed in Section 3.1.1 to determine the band-gap energy. This value is similar to the prediction [100]. Although the dip amplitude was as small as $\sim 0.015 \mathrm{mV}$, the absorption hence represented the band-gap. 


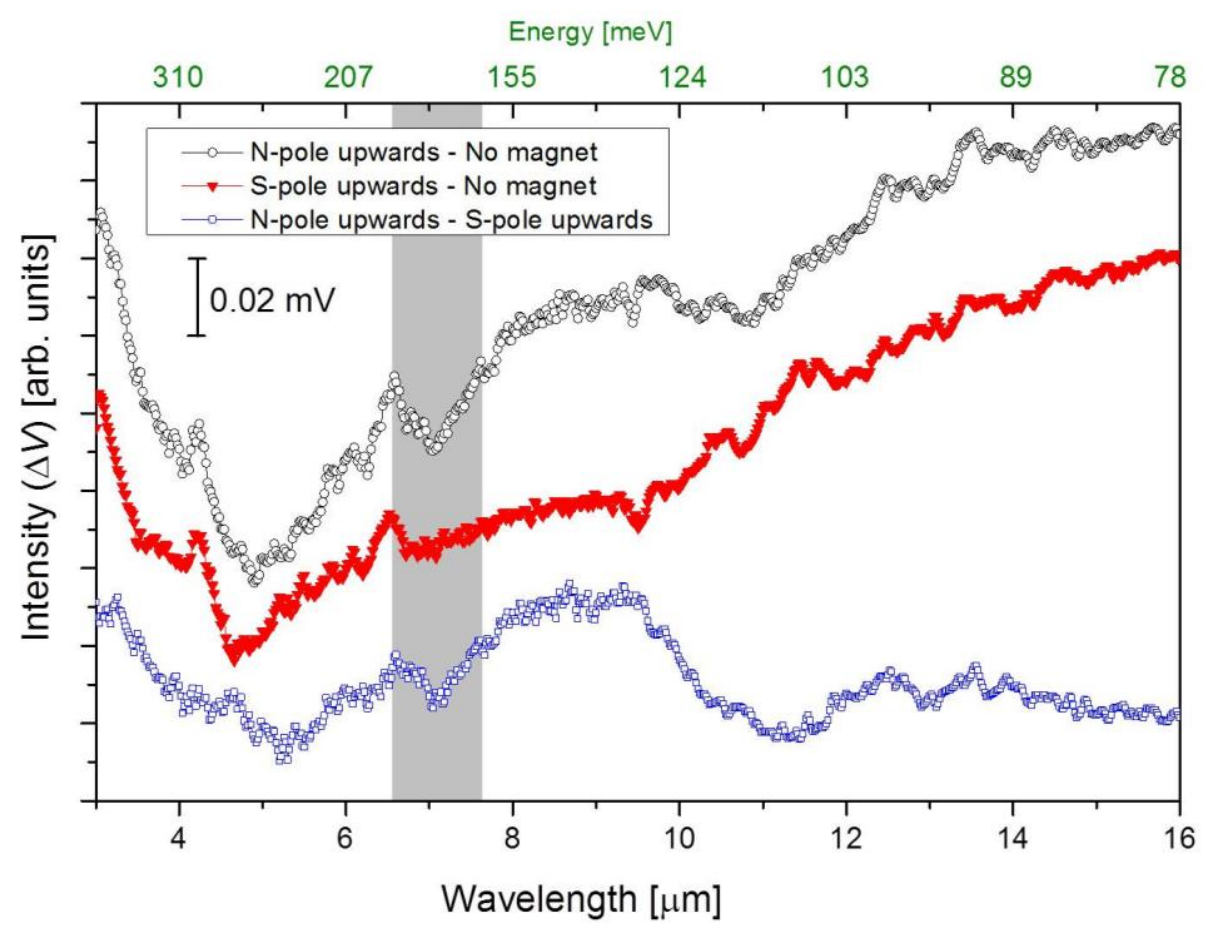

Fig. 7.1: Measured intensities for the stoichiometric bilk NiMnSb without a field and with fields with $\mathrm{N}$ and S-pole upwards in the wavelength range between 3 and $16 \mu \mathrm{m}$.

The small dip amplitude can be attributed to the surface roughness of the sample. The majority area of the sample surface is almost flat but with little dents. The sample surface also consists of some cavities that cover the area of $\sim 3 \mathrm{~mm}^{2}$ with a few micrometers depth (see Fig. 7.2), which are created during the fabrication and chemical polishing processes. These uneven surface causes scattering to the IR light and therefore reduces the amount of the reflected photons.

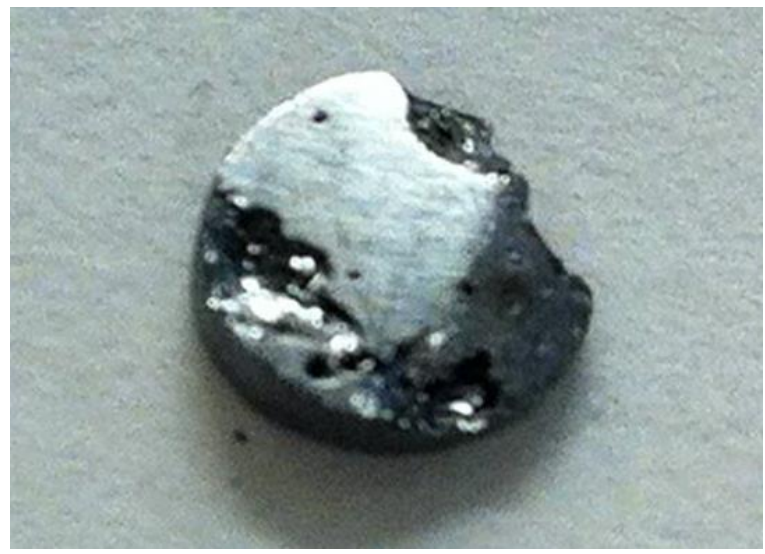

Fig. 7.2: Photograph of the bulk NiMnSb sample. 


\subsubsection{Non-stoichiometric sample}

The band-gap measurements were also conducted with the same procedures as described above. The individual measurements under magnetic fields at both field directions showed some dips at $\sim \lambda=4.2 \mu \mathrm{m}$ and $\sim \lambda=6.8 \mu \mathrm{m}$ in both field directions (N- and S-pole upwards) as shown in Fig. 7.3. Again, the difference in the intensity signals was calculated. Unlike the stoichiometric sample, the intensity difference did not have specific features corresponding to a band-gap (blue line) in Fig. 7.3. Therefore, the dips at $\sim 4.2 \mu \mathrm{m}$ could be attributed to the artifact from the IR light source as similar feature was found in other films (e.g., CFS).

The absence of a half-metallic band-gap can be attributed to the nonstoichiometry of this sample. Hence, it is apparent that the stoichiometric compositions during the sample fabrication are critical to achieve the half-metallicity in NMS.

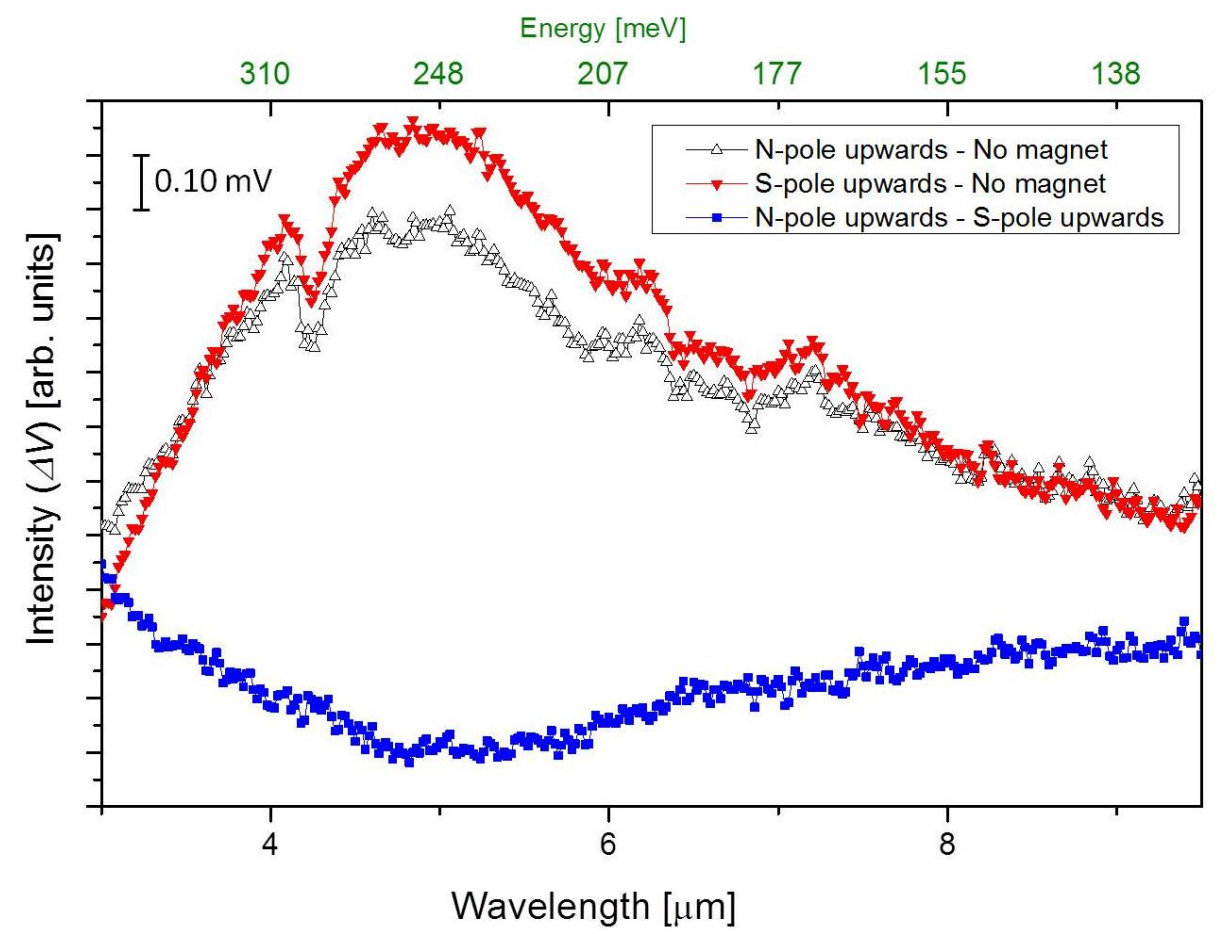

Fig. 7.3: Measured intensities for the non-stoichiometric bulk NiMnSb without a field and with fields with $\mathrm{N}$ - and $\mathrm{S}$-pole upwards in the wavelength range between 3 and $16 \mu \mathrm{m}$. 


\subsection{Summary}

The band-gap of single-crystal bulk NMS samples has been measured at RT using the developed IR technique. The stoichiometric sample shows a dip at around $\lambda=7 \mu \mathrm{m}$, corresponding to the energy band-gap of $\sim 0.175 \mathrm{eV}$. The dip is observed only with the field with the N-pole upwards, indicating only one spin band has a band-gap. The estimated value of the NMS band-gap is similar to the value predicted by the firstprinciples calculations [100].

On the other hand, the non-stoichiometric sample shows no notable feature, indicating that the non-stoichiometric sample do not have half-metallic behaviour. These results confirm that the IR technique to measure the band-gap is also applicable for the bulk samples due to the long penetration depth of the IR light $(\sim 20 \mu \mathrm{m})$ [70]. 


\section{Chapter 8}

\section{Conclusion and Future Work}

\subsection{Conclusion}

The characterisation of Heusler alloys, which are capable of realising $100 \%$ spin polarisation at RT as a HMF, is one of the avenues for the development of spintronic devices. The main purpose of this study is to develop a new technique to measure the band-gap of HMFs at RT. In order to achieve this objective, an experimental set-up has been designed utilising optical excitation. In this study, due to both its simplicity and viability, a reflection mode has been selected to measure the changes in the reflected signals from the sample surface by the presence of a band-gap. This set-up can also be used to measure the band-gap electrically by detecting the change in the four-pointprobe signals under the optical excitation.

In this study, circularly-polarised IR light was used to excite the minority spin electrons in Heusler-alloy films. The wavelength of the IR light was selected to be between 2 and $20 \mu \mathrm{m}$, which included the band-gap energy of the studied films. The studied alloys were $\mathrm{Co}_{2} \mathrm{FeSi}, \mathrm{Co}_{2} \mathrm{FeAl}_{0.5} \mathrm{Si}_{0.5}$ full-Heusler and $\mathrm{NiMnSb}$ half-Heusler alloys. The wavelength was controlled by a monochromator in order to select a specific wavelength range for each sample, as each one has different band-gap energy. In order 
to measure a small band-gap of the Heusler alloys in the order of a few meV, it was important to control in a narrow wavelength step, i.e., a $20 \mathrm{~nm}$ step. Although smaller steps of less than $1 \mathrm{~nm}$ could be used with a monochromator with higher accuracy, the $20 \mathrm{~nm}$ step was found to provide a clear intensity profile with low noise as the optimum condition. The IR light was then introduced onto the samples at $45^{\circ}$ normal to the plane. The reflected intensity was measured at different wavelengths with and without applying a perpendicular magnetic field $(\sim 2.2 \mathrm{kOe})$. By reversing the magnetic field whilst maintaining the circular polarisation of the IR light, the optical excitation became sensitive to the majority or minority spins, resulting in the difference between these signals to reveal the absorption in the light at the band-gap.

In order to validate this new technique, the band-gap was measured at RT for three different samples; polycrystalline CFS and epitaxial CFAS full-Heusler-alloy films and bulk single-crystal NMS half-Heusler alloy. These Heusler alloys were selected amongst hundreds of alloys because of their well-studied properties and their applicability for future spintronic devices. For example, CFS and CFAS were known to exhibit high $T_{\mathrm{C}}(>\mathrm{RT})$, while NMS and CFAS were known to possess large band-gaps and resulting high spin polarisation at RT.

In this study, polycrystalline CFS and epitaxial CFAS films were grown by HiTUS and UHV-MBE, respectively. The bulk single-crystal NMS samples were fabricated using the arc-melting method.

These samples were first characterised magnetically using a VSM. Their $M_{\mathrm{s}}$ were obtained from their $M-H$ hysteresis loops as $(634 \pm 72)$ and $(960 \pm 96) \mathrm{emu} / \mathrm{cm}^{3}$ for $\mathrm{CFS} / \mathrm{Si}$ and $\mathrm{CFS} / \mathrm{MgO}(001)$ films in their as-deposited states, respectively. These values were $\sim 52 \%$ and $\sim 70 \%$ of the theoretically predicted value, which indicated that the $\mathrm{CFS} / \mathrm{MgO}(001)$ films might be crystallised in the $B 2$ (and $A 2$ ) phase even without annealing. The CFAS showed larger $M_{\mathrm{s}}$ of $(1007 \pm 100) \mathrm{emu} / \mathrm{cm}^{3}$ in the as-deposited 
state, which is $92 \%$ of the theoretically predicted value. This indicated that these CFAS films should form the $L 2_{1}$ structures with minor $B 2$ or $A 2$ phases. The $M_{\mathrm{s}}$ of NMS was measured to be $4.01 \mu_{B} /$ f.u. $\left(=590 \mathrm{emu} / \mathrm{cm}^{3}\right)$, which is in good agreement with the expected values $\left(\sim 588 \mathrm{emu} / \mathrm{cm}^{3}\right)$.

The films were then characterised structurally using cross-sectional TEM and XRD. The TEM images with the corresponding electron diffraction patterns for the CFS films showed the polycrystalline nature and the formation of the $B 2$ and $L 2_{1}$ phases. The TEM images of the CFAS films showed that they have both the $L 2_{1}$ and $B 2$ phases, which agreed with RHEED patterns, demonstrating their epitaxial growth. The SAED further confirms the presence of the $B 2$ phase in the as-deposited state and possibly the $L 2_{1}$ phase after annealing at $400^{\circ} \mathrm{C}$ for up to $6 \mathrm{~h}$. The lattice constant of CFAS was calculated from the TEM images XRD measurements to be $0.5546 \mathrm{~nm}$, which is $\sim 98 \%$ of the reported bulk value. The formation of the $C 1_{b}$ crystalline structure for NMS was also confirmed by the XRD measurements.

After the confirmation of the quality of the samples by the above characterisation, their band-gaps were measured for their as-deposited and postannealed states. For CFS, the $E_{g}$ was measured to be $\sim 94 \mathrm{meV}$, which agreed very well with the calculated values of 100 and $146 \mathrm{meV}$ by density functional theory (DFT) and GGA, respectively. $E_{g}$ for CFAS was estimated to be $\sim 111 \mathrm{meV}$ for the as-deposited films, while it was reduced slightly to be $\sim 95 \mathrm{meV}$ for the post-annealed films up to 3 h. This also agreed with the calculated value of $100 \mathrm{meV}$ by DFT. It was found that the band-gap of both polycrystalline and epitaxial films vanished after annealing for $4.5 \mathrm{~h}$. This was attributed to the phase segregation as previously studied.

$E_{\mathrm{g}}$ of single-crystal bulk NMS samples was also measured at RT. The stoichiometric samples showed similar behaviour as the above films, in which the IR light was absorbed at specific wavelengths corresponding to the sample band-gap. $E_{g}$ 
for NMS was estimated to be $\sim 175 \mathrm{meV}$, which was again very close to the value predicted by the first-principles calculations (168 meV). Conversely, the nonstoichiometric samples did not demonstrate half-metallic behaviour and therefore no band-gap was observed.

These results confirm the capability of this newly developed technique as a means to measure half-metallic band-gaps for thin films as well as bulk samples at RT. Hence, the main objective of this study has been successfully achieved. This new technique has some notable advantages: (i) flexible design, in which the $E_{g}$ measurements can be conducted at various incident angles by modifying the set-up, (ii) high sensitivity in the order of few $\mu \mathrm{V}$ and (iii) applicability for both thin films and bulk half-metallic materials. This technique can therefore be used to analyse such halfmetallic materials very easily and can provide feedback to their growth and preparation process optimisation.

\subsection{Future Work}

Investigation of the surface regions of the films and bulk samples, in particular, the accurate measurement of the band-gap of the surface regions is of paramount importance to the half-metallic characterisation. This can be achieved by changing the IR incident angle $(\theta)$ from the sample plane. Due to the flexible design of the experimental set-up, the IR incident angle can be fully controlled between $1^{\circ} \leq \theta \leq 90^{\circ}$. The sample holder is installed on a precise rotary stage, which can be used to alter the angle to as low as $1^{\circ}$ without affecting the optical alignment. Such a low incident angle allows the measurements to be predominantly sensitive to the surface, while a large incident angle is sensitive to the entire films (penetration depth: $\sim 20 \mu \mathrm{m}$ ). By extrapolating these data, one can estimate the band-gap at the interface/surface and the bulk regions of the film. This can highlight the contributions of atomic disorder at the 
interface/surface on $E_{g}$. Such depth profile may provide direct feedback to the growth conditions lead to the enhancement in magnetoresistance in half-metallic Heusler-alloy junctions at RT.

As discussed earlier (see Chapter 2), the Heusler alloys have been predicted to possess larger $E_{g}$ at low temperature as compared with $E_{g}$ at RT. Therefore, it is interesting to perform further at low temperature on the same studied Heusler alloys. This can be achieved by using the cryostat with optical windows in the Electronics Department at the University of York. The cryostat has two optical windows, between which the sample can be held to allow optical access. However, this requires a longer optical path and consequently lower signal intensity. To overcome the signal-to-noise issues, it is necessary to replace the IR light source with a higher-power laser with wavelength tunability.

The band-gap can also be measured electrically by using four-point-probe method, which provides additional characterisation technique on the same set-up. In this method, electrical resistivity of the films is measured. It requires four in-line equally spaced push-pins to be placed in contact to the sample surface. The outer two pins are used to supply a current and the two inner pins are used to measure a voltage. Hence, the local resistance is obtained. With the optical excitation, photons with energy matched with the band-gap energy can be absorbed. Therefore, the absorbed photons at $E_{g}$ can increase the carriers in the Heusler-alloy samples and can hence increase the electrical signal in this measurement. This may also improve the signal-to-noise ratios for the band-gap determination. 


\section{Appendix 1}

\section{Block Diagram of the LabView}

In this section, a block diagram of the LabView programme is shown, which has been developed to control the band-gap measurement set-up. The programme has been developed with some help from Dr Ranjdar Abdullah at the Department of Electronics in the University of York. An interface of the programme is shown in Fig. A.1. 


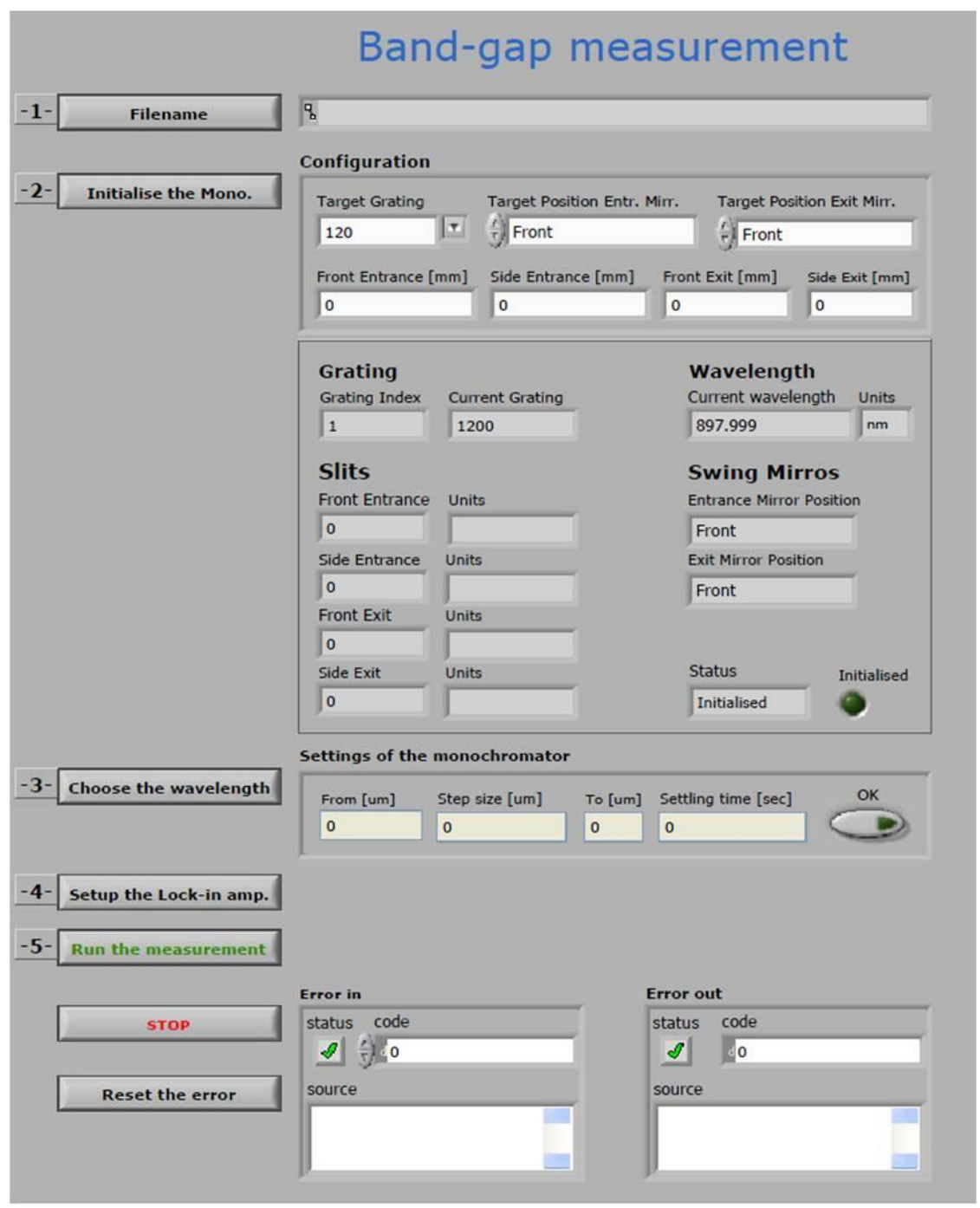

Fig. A.1: Interface of the LabView programme for the band-gap measurements.

The following figures show the block diagrams of the key steps to run the band-gap measurements. Figure A.2 shows the creation of the file, where the measured data are stored. The monochrometer is then initialised as shown in Fig. A.3, followed by the setup of the lock-in amplifier as appeared in Fig. A.4. The parameters are also selected as depicted in Fig. A.5. Finally, Figure A.6 shows the execution of the measurements. 


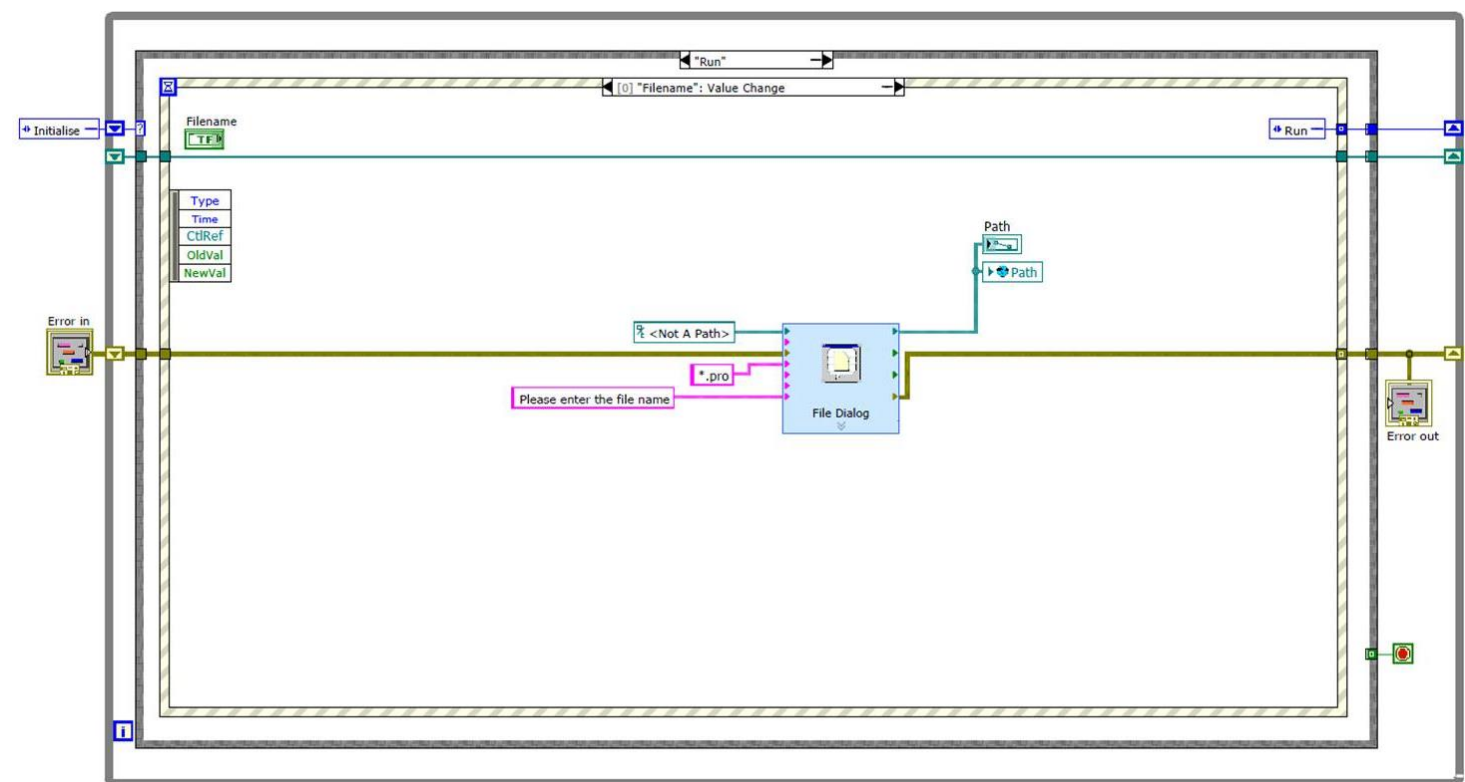

Fig. A.2: Block diagram of the first step of the band-gap measurement to create a file to save the measured data.

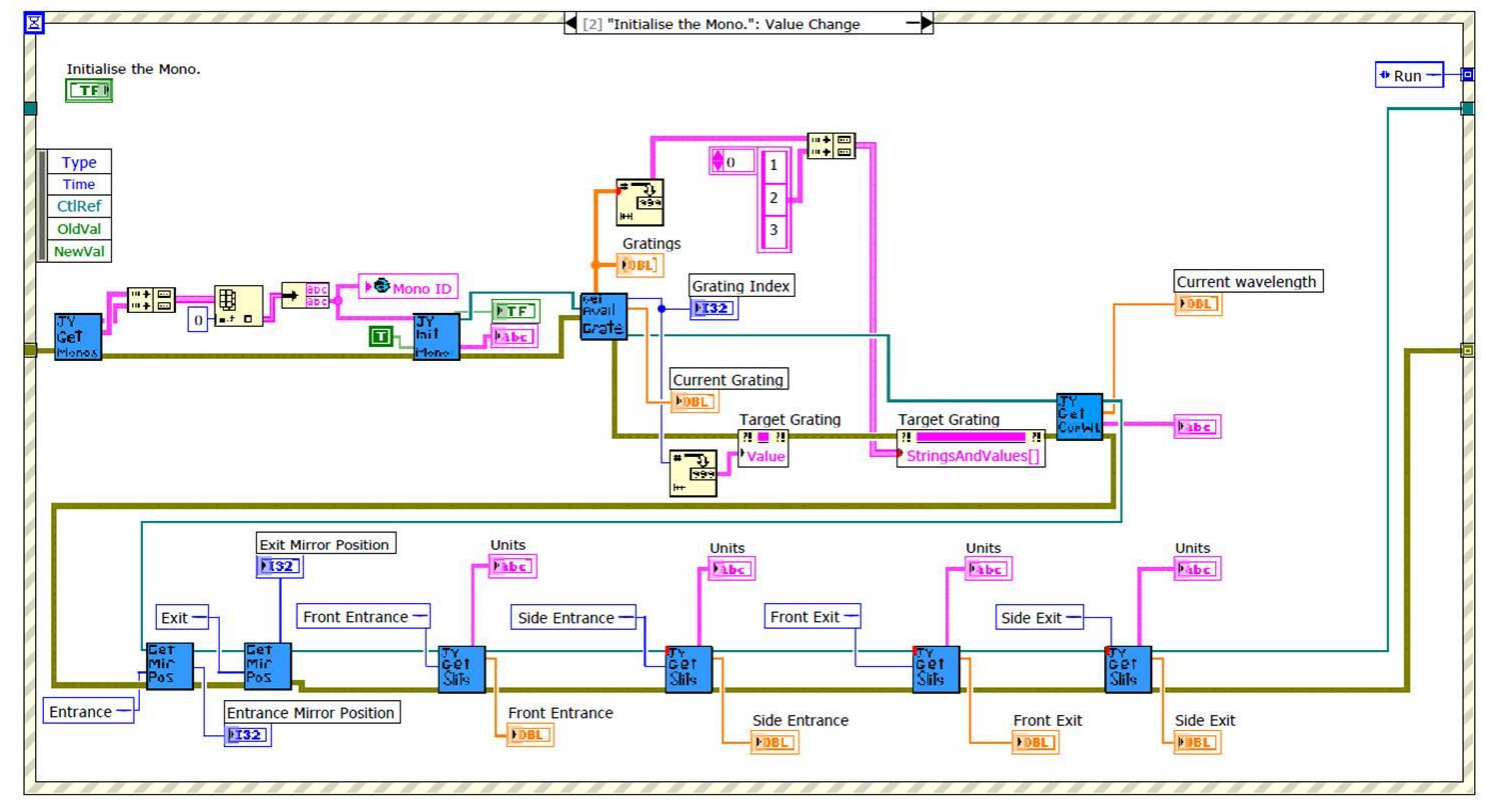

Fig. A.3: Block diagram of the initialisation of the monochromator. 


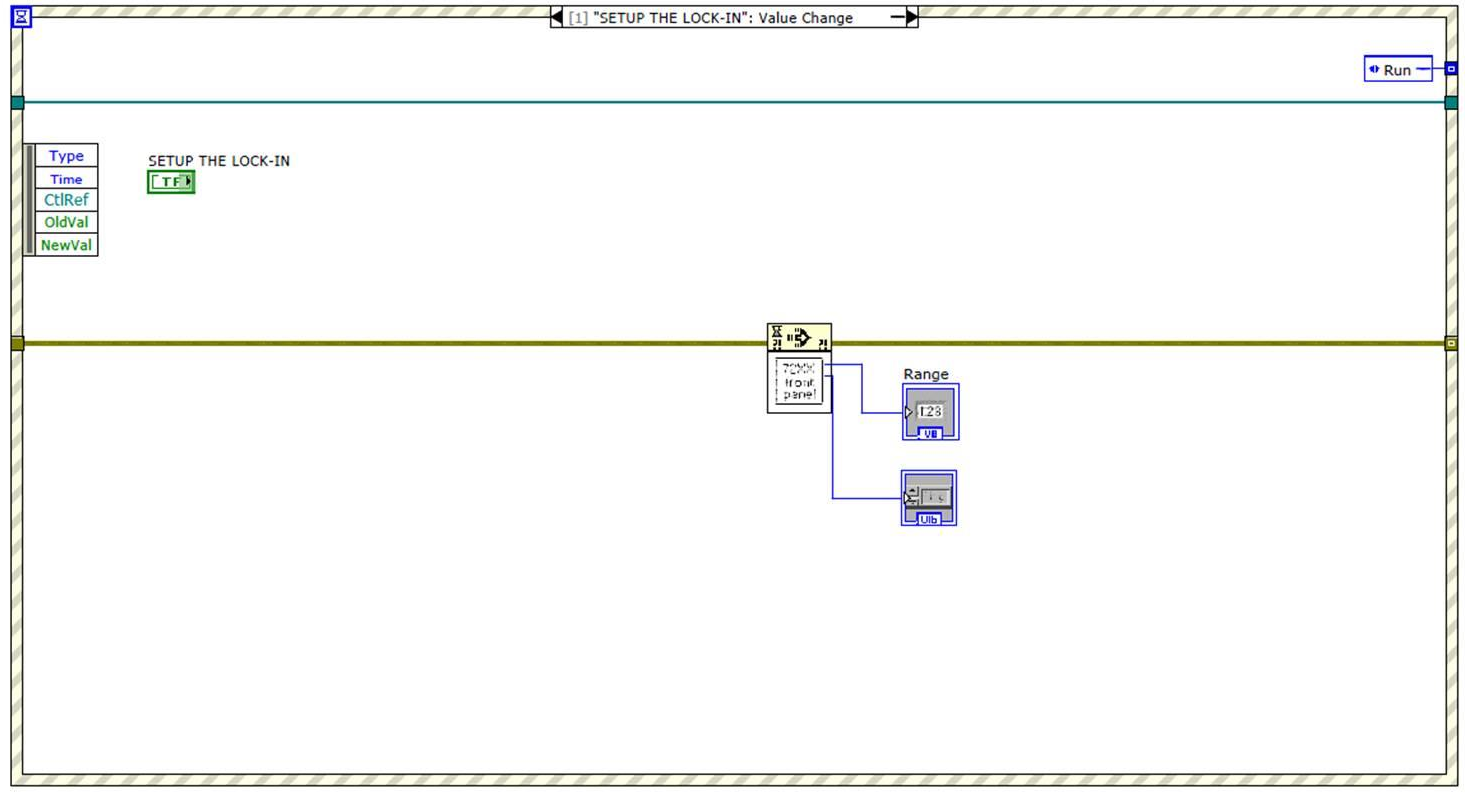

Fig. A.4: Block diagram of the set-up of the lock-in amplifier.

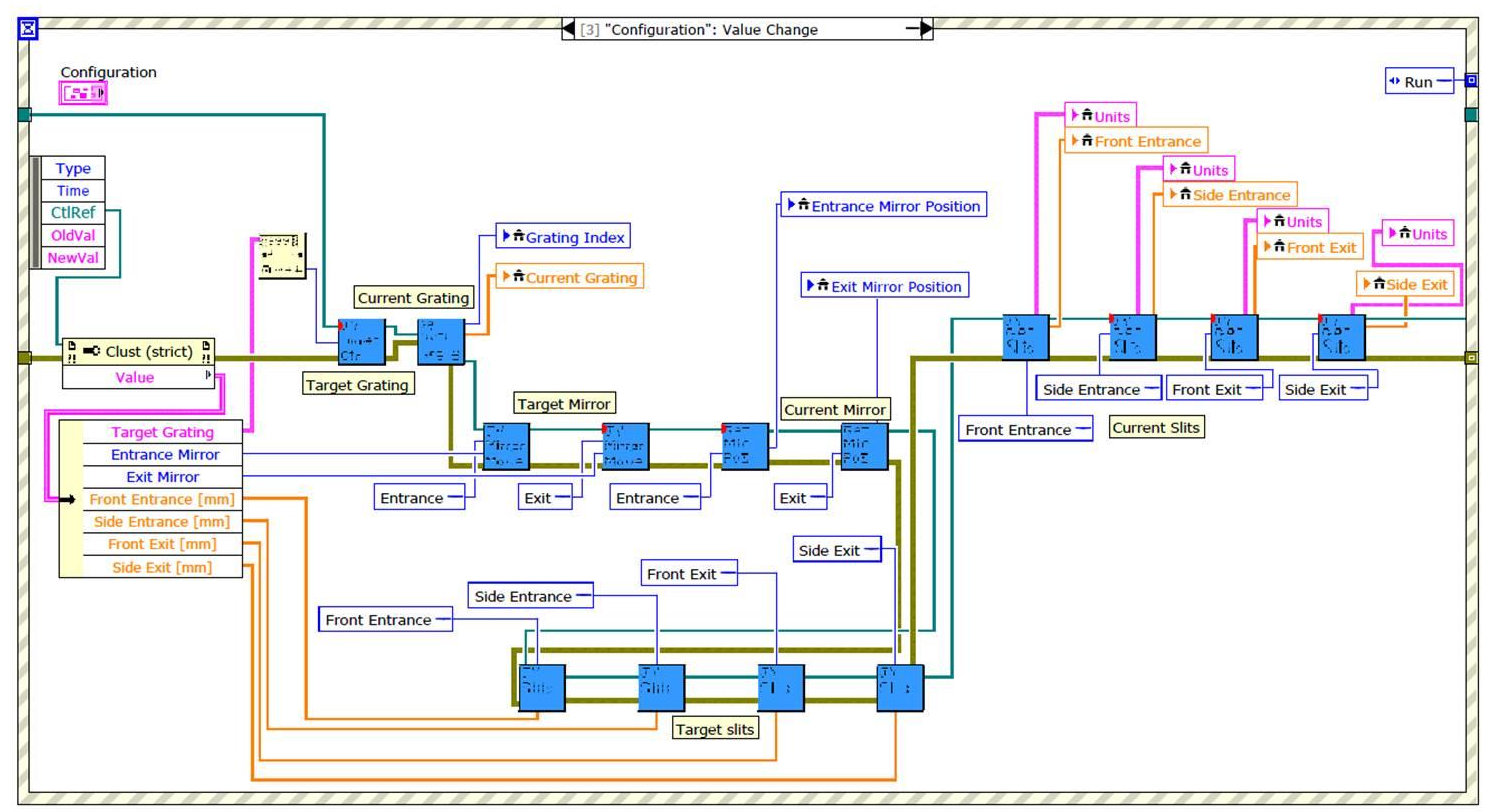

Fig. A.5: Block diagram of the selection of the parameter values for the lock-in amplifier. 


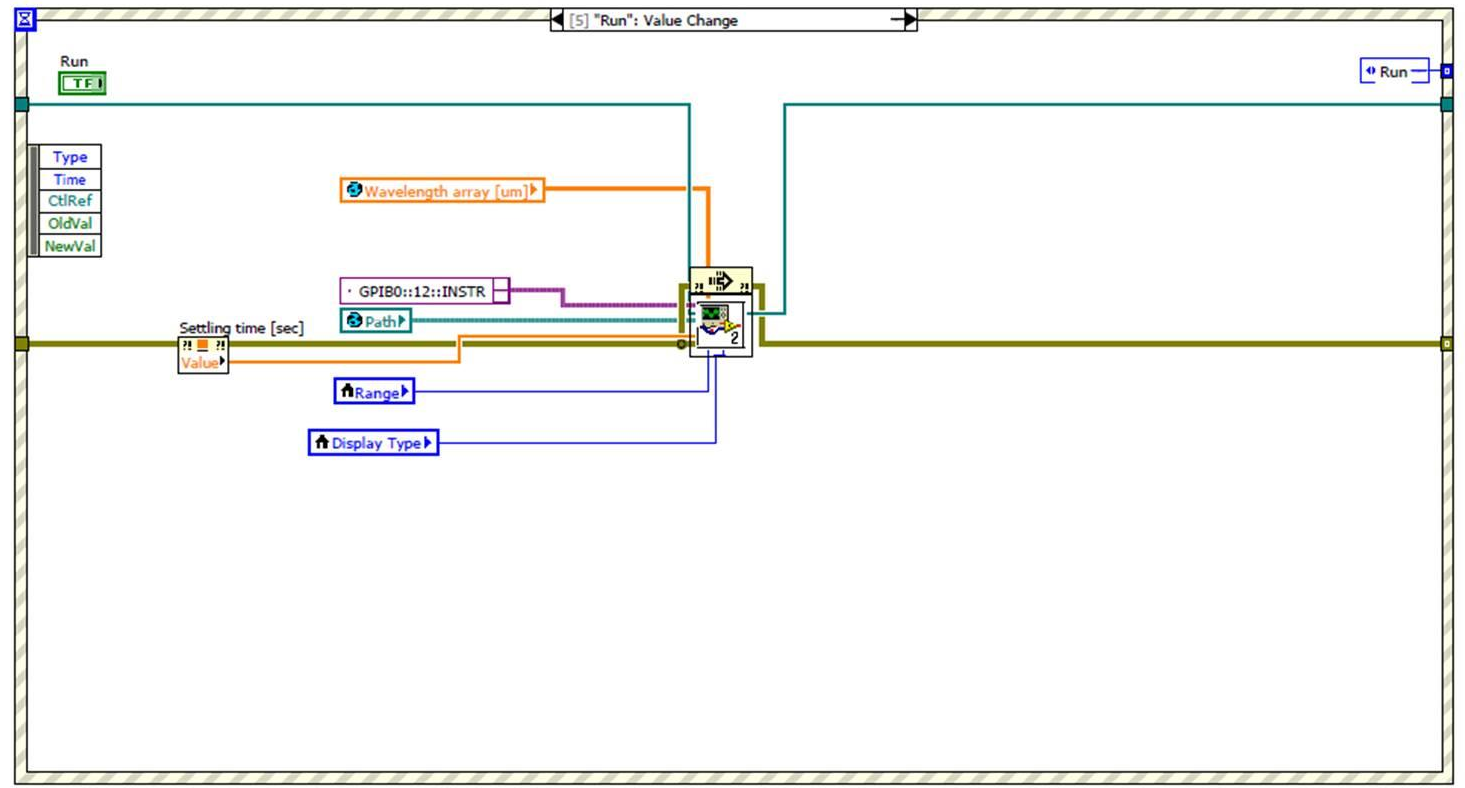

Fig. A.6: Block diagram of the execution of the measurement with the control of all the devices connected. 


\section{Appendix 2}

\section{Electronic Circuit}

In this section, the diagram of an electronic circuit is shown. It has been designed to reduce the noise level of the photodetector and accordingly to enhance the output signals. After several electronic circuits being tested, the circuit as show in in B.1 has been found to reduce the noise from $>35 \mathrm{mV}$ to around $20 \mathrm{mV}$. Here, the use of different resistors and capacitors reduces the electromagnetic radiations around the photodetector. The capacitors are also more convenient to be connected and soldered than an integrated circuit with a minimum of 8 pins and requirement of additional components, such as resistors. Therefore this circuit has been fabricated and employed in this study.

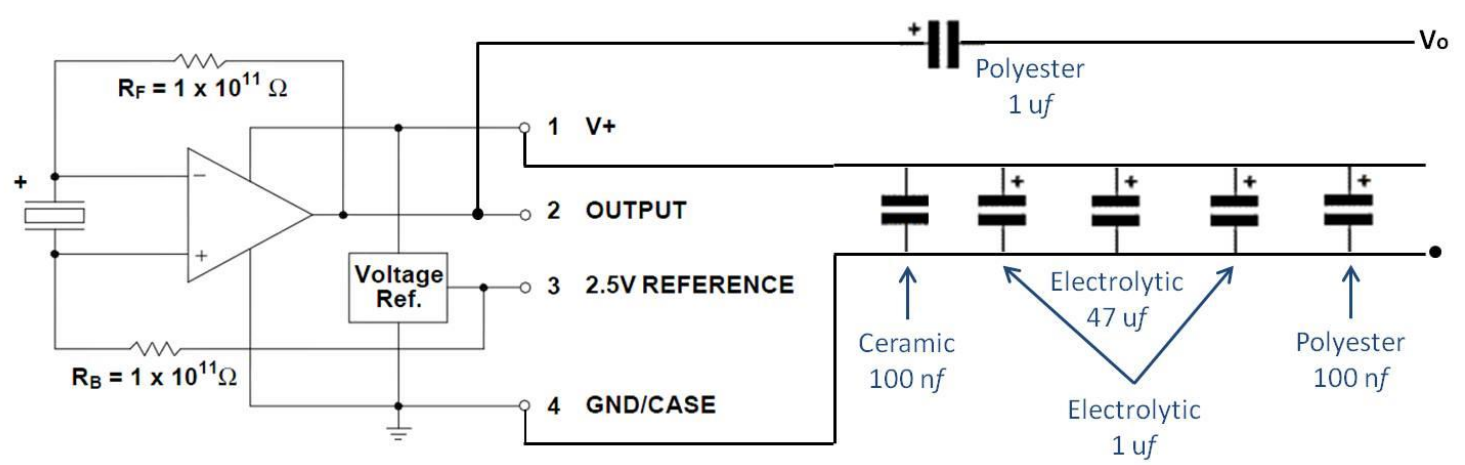

Fig. B.1: Final electronic circuit, which has been developed in this study. 


\section{List of Symbols}

\begin{tabular}{|c|c|}
\hline$\mu_{\mathrm{B}} /$ f.u. & Bohr magneton / formula unit \\
\hline$a$ & Lattice constant \\
\hline$A_{\mathrm{c}}$ & Cross-sectional area of the coil \\
\hline AP & Antiparallel configuration \\
\hline$B_{\mathrm{m}}$ & Magnetic flux density \\
\hline$c$ & Speed of light \\
\hline$D^{*}$ & Detectivity \\
\hline E & Energy of photon \\
\hline e.m.f. & Electromotive force \\
\hline$E_{\mathrm{F}}$ & Fermi level \\
\hline$E_{\mathrm{g}}$ & Band-gap \\
\hline$e_{g}$ & degenerate antibonding states \\
\hline$f$ & Frequency \\
\hline$G$ & Tunnelling conductance \\
\hline$H$ & Magnetic field \\
\hline$h$ & Planck's constant \\
\hline$H_{\mathrm{c}}$ & Coercivity \\
\hline$k_{\mathrm{B}} T$ & Thermal stability \\
\hline$m$ & Magnetic moment \\
\hline$M$ & Magnetisation \\
\hline$M_{\mathrm{s}}$ & Saturation magnetisation \\
\hline$M_{\mathrm{t}}$ & Magnetic moment \\
\hline
\end{tabular}




\begin{tabular}{|c|c|}
\hline$N_{\uparrow}\left(N_{\downarrow}\right)$ & Densities of the majority and (minority) electrons \\
\hline $\mathrm{P}$ & Parallel configuration \\
\hline$P$ & Spin polarisation \\
\hline $\mathrm{Pd}$ & Palladium \\
\hline$R$ & Resistance \\
\hline$R_{\mathrm{AP}}$ & Resistance for antiparallel configuration \\
\hline $\mathrm{RF}$ & Radio frequency \\
\hline$R_{\max }$ & Maximum resistance \\
\hline$R_{\min }$ & Minimum resistance \\
\hline$R_{\mathrm{P}}$ & Resistance for parallel configuration \\
\hline$t_{2 g}$ & degenerate bonding states \\
\hline$T_{\mathrm{C}}$ & Curie temperature \\
\hline$Z_{\mathrm{t}}$ & Valence electrons \\
\hline$\Delta V$ & Intensity difference between $S$ - and N-pole upwards \\
\hline$\lambda$ & Wavelength \\
\hline$\phi_{\mathrm{m}}$ & Magnetic flux \\
\hline$\varepsilon$ & Induced voltage \\
\hline
\end{tabular}




\section{List of Abbreviations}

$1 \mathrm{~T}$

2DEG

4MTJ

AR

CCD

CFAS

CFS

CIP

CPP

DFT

DMS

DOS

DRAM

EDX

EELS

FM

FWHM

GGA

GMR

HDD

HiTUS

HMF
One-transistor

Two-dimensional electron gas

Four MRAM cells

Andreev reflection

Charge-coupled device

$\mathrm{Co}_{2} \mathrm{FeAl}_{0.5} \mathrm{Si}_{0.5}$

$\mathrm{Co}_{2} \mathrm{FeSi}$

Current in the plane

Current perpendicular to the plane

Density functional theory

Dilute magnetic semiconductor

Density of states

Dynamic random access memory

Energy-dispersive X-ray spectroscopy

Electron energy-loss spectroscopy

Ferromagnet

Full width half maximum

Generalised gradient approximation

Giant magnetoresistance

Hard disk drives

High target utilisation sputtering

Half-metallic ferromagnet 
IR

LDA

$\mathrm{LDA}+U$

LEED

LKKR-CPA

LSMO

MBE

MRAM

MTJ

NMS

PCAR

PLD

RHEED

RT

RTA

SAED

SC

Spin-FET

SPIP

SPPA

SRPES

STT-MRAM
Infrared

Local density approximation

Local density approximation with Coulomb interactions

Low energy electron diffraction

Layer Korringa-Kohn-Rostoker method in conjunction with the coherent potential approximation

$\mathrm{LaSrMnO}_{3}$

Molecular beam epitaxy

Magnetic random access memory

Magnetic tunnel junction

$\mathrm{NiMnSb}$

Point contact Andreev reflection

Pulsed laser deposition

Reflection high-energy electron diffraction

Room temperature

Rapid thermal annealing

Selected area electron diffraction

Semiconductor

Spin-polarised field effect transistor

Spin-polarised inverse photoemission

Spin-polarised positron-annihilation

Spin-resolved photoemission technique

Spin-transfer torque MRAM 
TEM

TMR

UHV

VSM

XRD
Transmission electron microscopy

Tunnelling magnetoresistance

Ultrahigh vacuum

Vibrating sample magnetometer

X-ray diffraction 


\section{References}

[1] S. A. Wolf, D. D. Awschalom, R. A. Buhraman, J. M. Daughton, S. von Molnar, M. L. Roukes, A. Y. Chtchelkanova and D. M. Treger, Science 294, 1488 (2001).

[2] S. S. Das J. Fabian, X. Hu and I. Zutic, IEEE Trans. Magn. 36, 2821 (2000).

[3] A. Hirohata and K. Takanashi, J. Phys. D: Appl. Phys. 47, 193001 (2014).

[4] C. Felser and G. H. Fecher (Eds.), "Spintronics: From Materials to Devices" (Springer, Heidelberg, 2013) pp 1-98.

[5] C. Felser, G. H. Fecher and B. Balke, Angew. Chem. Int. Ed. 46, 668 (2007).

[6] A. Hirohata, M. Kikuchi, N. Tezuka, K. Inomata, J. S. Claydon, Y. B. Xu and G. van der Laan, Curr. Opin. Solid State Mater. Sci., 10, 93 (2006).

[7] R. J. Soulen Jr., J. M. Byers, M. S. Osofsky, B. Nadgorny, T. Ambrose, S. F. Cheng, P. R. Broussard, C. T. Tanaka, J. Nowak, J. S. Moodera, A. Barry and J. M. D. Coey, Science, 282, 85 (1998).

[8] R. Meservey and P. M. Tedrow, Phys. Rep., 238, 173 (1994).

[9] Y. S. Dedkov, M. Fonine, C. König, U. Rüdiger and G. Güntherodt, Appl. Phys. Lett., 80, 4181 (2006).

[10] G. M. Müller, J. Walowski, M. Djordjevic, G.-X Miao, A. Gupta, A. V. Ramos, K. Gehrke, V. Moshnyaga, K. Samwer, J. Schmalhorst, A. Thomas, A. Hütten, G. Reiss, J. S. Moodera and M. Münzenberg, Nature Mater., 8, 56 (2009).

[11] K. Inomata, N. Ikeda, N. Tezuka, R. Goto, S. Sugimoto, M. Wojcik and E. Jedryka, Sci. Technol. Adv. Mater. 9, 014101 (2008).

[12] G. Schmidt, D. Ferrand, L. W. Molenkamp, A. T. Filip and B. J. van Wees, Phys Rev B 62, R4790 (2000).

[13] R. A. de Groot, F. M. Mueller, P. G. van Engen and K. H. J. Buschow, Phys. Rev. Lett. 50, 2024 (1983).

[14] H. Akinaga, T. Manago and M. Shirai, J. J. Appl. Phys. 39, L1118 (2000).

[15] Y. Okimoto, T. Katsufuji, T. Ishikawa, A. Urushibara, T. Arima and Y. Tokura, Phys. Rev. Lett. 75, 109 (1995).

[16] K. Schwarz, J. Phys. F: Met. Phys. 16, L211 (1986).

[17] I. Galanakis and Ph. Mavropoulos, Phys. Rev. B 67, 104417 (2003).

[18] J. Kübler, Phys. Rev. B 67, 220403(R) (2003).

[19] D. C. Worledge and T. H. Geballe, App. Phys. Lett. 76, 900 (2000).

[20] J.-H. Park, E. Vescovo, H. -J. Kim, C. Kwon, R. Ramesh and T. Venkatesan, Phys. Rev. Lett. 81, 1953 (1998).

[21] G. P. Singh, S. Ram, J. Eckert and H. -J. Fecht, J. Phys.: Conf. Ser. 144, 012110 (2009).

[22] B. R. Maddox, C. S. Yoo, D. Kasinathan, W. E. Pickett and R. T. Scalettar, Phys. Rev. B 73, 144111 (2006).

[23] Y. Ji, G. J. Strijkers, F. Y. Yang, C. L. Chien, J. M. Byers, A. Anguelouch, G. Xiao and A. Gupta, Phys. Rev. Lett. 86, 5585 (2001).

[24] J. D. Aldous, C. W. Burrows, A. M. Sánchez, R. Beanland, I. Maskery, M. K. Bradley, M. dos S. Dias, J. B. Staunton and G. R. Bell, Phys. Rev. B 85, 060403(R) (2012).

[25] F. Heusler, w. Starck, E. Haupt, Verh. Dtsch. Phys. Ges. 5, 220 (1903).

[26] T. M. Nakatani, N. Hase, H. S. Goripati, Y. K. Takahashi, T. Furubayashi and K. Hono, IEEE Trans. Magn. 48, 1751 (2012). 
[27] M. Vahidi, J. A. Gifford, S. K. Zhang, S. Krishnamurthy, Z. G. Yu, L. Yu, M. Huang, C. Youngbull, T. Y. Chen and N. Newman, Appl. Phys. Lett. Mat. 2, 046108 (2014).

[28] R. Shan, H. Sukegawa, W. H. Wang, M. Kodzuka, T. Furubayashi, T. Ohkubo, S. Mitani, K. Inomata and K. Ohno, Phys. Rev. Lett. 102, 246601 (2009).

[29] I. Galanakis, Ph. Mavropoulos and P. H. Dederichs, J. Phys. D: Appl. Phys. 39, 765 (2006).

[30] I. Galanakis and P. H. Dederichs, Phys. Rev. B 66, 174429 (2002).

[31] C. Kittel, "Introduction to Solid State Physics" (John Wiley \& Sons, New Jersey, 2005) pp 429-431.

[32] N. W. Ashcroft and N. D. Mermin, "Solid State Physics" (Holt-S Aunders, Japan, 1981) pp. 776-779.

[33] V. Kumar, S. K. Sharma, T. P. Sharma and V. Singh, Opt. Materi. 12, 115 (1999).

[34] P. M. Tedrow and R. Meservey, Phys. Rev. Lett. 26, 192 (1971).

[35] P. M. Tedrow and R. Meservey, Phys. Rev. Lett. 27, 919 (1971).

[36] P. M. Tedrow and R. Meservey, Phys. Rev. B 7, 318 (1973).

[37] M. Julliere, Phys. Lett. A 54, 225 (1975).

[38] S. M. Thompson, J. Phys. D: Appl. Phys. 41, 093001 (2008).

[39] T. Miyazaki and N. Tezuka. J. Magn. Magn. Mater. 139, L231 (1995).

[40] J. S. Moodera, L. R. Kinder, T. M. Wong and R. Meservey, Phys. Rev. Lett. 74, 3273 (1995).

[41] W. H. Butler, X.-G. Zhang, T. C. Schulthess and J. M. Maclaren, Phys Rev. B 63, 054416 (2001).

[42] J. Mathon and A. Umerski, Phys. Rev. B 63, 220403 (2001).

[43] J. Hayakawa, S. Ikeda, Y. M. Lee, F. Matsukura and H. Ohno, App. Phys. Lett. 89, 232510 (2006).

[44] S. Ikeda, J. Hayakawa, Y. Ashizawa, Y. M. Lee, K. Miura, H. Hasegawa, M. Tsunoda, F. Matsukura and H. Ohno, App. Phys. Lett. 93, 082508 (2008).

[45] M. N. Baibich, J. M. Broto, A. Fert, F. N. Van Dau, F. Petroff, P. Etienne, G. Creuzet, A. Friederich and J. Chazelas, Phys. Rev. Lett. 61, 2472 (1988).

[46] G. Binasch, P. Grünberg, F. Saurenbach and W. Zinn, Phys. Rev. B 39, 4828 (1989).

[47] J. Bass and W. P. Pratt, J. Magn. Magn. Mater. 200, 274 (1999).

[48] E. Y. Tsymbal and I. Zutic (Eds.), "Handbook of Spin Transport and Magnetism" (CRC, New York, 2012) p 9.

[49] S. Dubois, L. Piraux, J. George, K. Ounadjela, J. Duvail and A. Fert, Phys. Rev. B 60, 477 (1999).

[50] C. Vouille, A. Fert, A. Barthélémy, S. Y. Hsu, R. Loloee and P. A. Schroeder, J. Appl. Phys. 81, 4573 (1997).

[51] T. Kimura, J. Hamrle and Y. Otani, Phys. Rev. B 72, 014461 (2005).

[52] J. Bass and W. P. P. Jr, J. Phys.: Condens. Matter 19, 183201 (2007).

[53] Y. Huai, AAPPS Bulletin 18, 33 (2008).

[54] https://www.everspin.com/64mb-spin-torque-mram-ddr3-dram-compatible.

[55] I. L. Prejbeanu, S. Bandiera, J. A-Hérault, R. C. Sousa, B. Dieny and J-P. Nozières, J. Phys. D: Appl. Phys. 46, 074002 (2013).

[56] A. V. Khvalkovskiy, D. Apalkov, S. Watts, R. Chepulskii, R. S. Beach, A. Ong., X. Tang, A. D-Smith, W. H. Butler, P. B. Visscher, D. Lottis, E. Chen, V. Nikitin and M. Krounbi, J. Phys. D: Appl. Phys. 46, 074001 (2013).

[57] T. Kawahara, R. Takemura, K. Miura, J. Hayakawa, S. Ikeda, Y. Lee, R. Sasaki, Y. Goto, K. Ito, T. Meguro, F. Matsukura, H. Takahashi, H. Matsuoka and H. Ohno, 2007 ISSCC Technical Digest (2007) p 480. 
[58] S. Datta and B. Das, Appl. Phys. Lett. 56, 665 (1990).

[59] K. Inomata, "Magnoresistive random access memoeries (mrams)", http://www.nims.go.jp/mmu/tutorials/SpinFET.html (2015).

[60] L. R. Fleet, Interface and Transport Properties of $\mathrm{Fe} / \mathrm{GaAs}(001)$ Heterostructures, PhD thesis, Department of Physics, University of York (2012).

[61] J.-H. Park, E. Vescovo, H.-J. Kim, C. Kwon, R. Ramesh and T. Venkatesan, Nature 392, (1998).

[62] S. Wurmehl, G. H. Fecher, H. C. Kandpal, V. Ksenofontov, C. Felser, H.-Ji Lin and J. Morais, Phys. Rev. B 72, 184434 (2005).

[63] S. Wurmehl, G. H. Fecher, H. C. Kandpal, V. Ksenofontov, C. Felser and H.-Ji Lin, Appl. Phys. Lett. 88, 032503 (2006).

[64] Z. Gercsi, A. Rajanikanth, Y. K. Takahashi, K. Honob, M. Kikuchi, N. Tezuka and K. Inomata, Appl. Phys. Lett. 89, 082512 (2006).

[65] X.-Q. Chen, R. Podloucky and P. Rogl, J. Appl. Phys. 100, 113901 (2006).

[66] H. C. Kandpal, G. H Fecher and C. Felser, J. Phys. D: Appl. Phys. 40, 1507 (2007).

[67] H. C. Kandpal, G. H. Fecher, C. Felser and G. Schönhense, Phys. Rev. B 73, 094422 (2006).

[68] N. P. Aley, S. Takayama, A. Hirohata and K. O'Grady, IEEE Trans. Magn. 47, 10 (2011).

[69] V. Niculescu, T. J. Burcil, K. RAJ and J. 1. Budnick, J. Magn. Magn. Mater. 5, 60 (1977).

[70] B. Peters, A. Alfonsov, C. G. F. Blum, S. J. Hageman, P. M. Woodward, S. Wurmehl, B. Büchner and F. Y. Yang, Appl. Phys. Lett. 103, 162404 (2013).

[71] A. Hirohata and Y. Otani (Eds.), "Epitaxial Ferromagnetic Films and Spintronic Applications" (Research Signpost, Kerala, 2009) pp 214-217.

[72] N. Tezuka, N. Ikeda, A. Miyazaki, S. Sugimoto, M. Kikuchi and K. Inomata, Appl. Phys. Lett. 89, 112514 (2006).

[73] H. Sukegawa, W. Wang, R. Shan, T. Nakatani, K. Inomata and K. Hono, Phys. Rev. B 79, 184418 (2009).

[74] W. Wang, H. Sukegawa, R. Shan and K. Inomata, Appl. Phys. Lett. 93, 1825004 (2008).

[75] S. Trudel, G. Wolf, J. Hamrle, B. Hillebrands, P. Klaer M. Kallmayer, H.-J. Elmers, H. Sukegawa, W. Wang and K. Inomata, Phys. Rev. B 83, 104412 (2011).

[76] T. M. Nakatani, A. Rajanikanth, Z. Gercsi, Y. K. Takahashi, K. Inomata and K. Hono, J. Appl. Phys. 102, 033916 (2007).

[77] B. Balke, G. H. Fecher and C. Felser, Appl. Phys. Lett. 90, 242503 (2007).

[78] G. H. Fecher and C. Felser, J. Phys. D: Appl. Phys. 40, 1582 (2007).

[79] N. Tezuka, N. Ikeda, F. Mitsuhashi and S. Sugimoto, J. Appl. Phys. 105, 07C925 (2009).

[80] T. M. Nakatani, Y. Du, Y. K. Takahashi, T. Furubayashi and K. Hono, Acta. Mat. 61, 3695 (2013).

[81] L. M. Loong, J. H. Kwon, P. Deorani, C. N. T. Yu, A. Hirohata and H. Yang, Appl. Phys. Lett. 104, 232409 (2014).

[82] A. Hirohata, K. Izumida, S. Ishizawa, J. Sagar and T. Nakayama, J. Appl. Phys. 115, 17D725 (2014).

[83] C. N. Borca, D. Ristoiu, T. Komesu, A. N. Caruso, J. Pierre, L. Ranno, J. P. Nozieres and P. Dowben, J. Phys.: Condens. Matter 19, 315211 (2007).

[84] A. Koveshnikov, G. Woltersdorf, J. Q. Liu, B. Kardasz, O. Mosendz, B. Heinrich, K. L. Kavanagh, P. Bach, A. S. Bader, C. Schumacher, C. Rüster, C. Gould, G. Schmidt, L. W. Molenkamp and C. Kumpf, J. Appl. Phys. 97, 073906 (2005). 
[85] S. Gardelis, J. Androulakis, P. Migiakis, J. Giapintzakis, S. K. Clowes, Y. Bugoslavsky, W. R. Branford, Y. Miyoshi and L. F. Cohen, J. Appl. Phys. 95, 8063 (2004).

[86] L. Ritchie, G. Xiao, Y. Ji, T. Y. Chen, C. L. Chien, M. Zhang, J. Chen, Z. Liu, G. Wu and X. X. Zhang, Phys. Rev. B 68, 104430 (2003).

[87] T. Ryba, Z. Vargova, R. Varga, S. Ilkovic, M. Reiffers, V. Haskova, P. Szabo,. J. Kravcak and R. Gyepes, Acta Phys. Polonica A, 126, 206 (2014).

[88] S. Gardelis, J. Androulakis, O. Monnereau, P. D. Buckle and J. Giapintzakis, J. Phys.: Conference Series 10, 167 (2005).

[89] S. Gardelis, J. Androulakis, J. Giapintzakis, O. Monnereau and P. D. Buckle, Appl. Phys. Lett. 85, 3178 (2004).

[90] C. Hordequin, J. Pierre and R. Currat, J. Magn. Magn. Mater. 162, 75 (1996).

[91] D. Orgassa, H. Fujiwara, T. C. Schulthess and W. H. Butler, J. Appl. Phys. 87, 5870 (2000).

[92] K. E. H. M. Hanssen and P. E. Mijnarends, Phys. Rev. B 34, 5009 (1986).

[93] D. Ristoiu, J. P. Nozières, C. N. Borca, T. Komesu, H.-k. Jeong and P. A. Dowben, Europhys. Lett. 49, 624 (2000).

[94] S. K. Clowes, Y. Miyoshi, Y. Bugoslavsky, W. R. Branford, C. Grigorescu, S. A. Manea, O. Monnereau and L. F. Cohen, Phys. Rev. B 69, 214425 (2004).

[95] G. L. Bona, F. Meier, M. Taborelli, E. Bucher and P.H. Schrnidt, Solid State Commun. 56, 391 (1985).

[96] W. Zhu, B. Sinkovic, E. Vescovo, C. Tanaka and J. S. Moodera, Phys. Rev. B 64, 060403 (2001).

[97] Ph. Mavropoulos, I. Galanakis, V. Popescu and P. H. Dederichs, J. Phys.: Condens. Matter 16, S5759 (2004).

[98] Ph. Mavropoulos, K. Sato, R. Zeller and P. H. Dederichs, Phys. Rev. B 69, 054424 (2004).

[99] N. A. Tuan and N. P. Duong, Appl. Phys. Lett. 99, 162507 (2011).

[100] Y. Wu, B. Wu, Z. Wei, Z. Zhou, C. Zhao, Y. Xiong, S. Tou, S. Yang, B. Zhou, Y. Shao, Intermetallics 53, 26 (2014).

[101] M. M. Kirillova, A. A. Makhnev, E. I. Shreder, V. P. Dyakina and N. B. Gorina, Phys. Stat. Sol. (b) 187, 231 (1995).

[102] S. J. Jenkins, Phys. Rev. B 70, 245401 (2004).

[103] S. J. Jenkins and D. A. King, Surf. Sci. 494, L793 (2001).

[104] LOT-Oriel website: http://www.lotoriel.com/files/downloads/lightsources/eu/LQ_10 30_W_IR-sources_eu.pdf

[105] Horiba website: www.horiba.com/fileadmin/uploads/Scientific/Documents/Mono/iHR.pdf

[106] Horiba website: www.horiba.com/

[107] Edmund Optics, http://www.edmundoptics.com/optics/polarizers/wire-gridpolarizers/infrared-ir-wire-grid-polarizers/62-774

[108] Edmund Optics, data sheet attached with the polariser.

[109] II-VI Infrared, http://www.iiviinfrared.com/CO2-Laser-Optics/prismsrhombs.html

[110] II-VI Infrared, data sheet attached with the $\lambda / 4$-wave plate.

[111] http://irassociates.com/index.php?page=hgcdte

[112] http://www.alrad.co.uk/photonics/Photodetectors.html

[113] http://www.roithner-laser.com/pd_midir_standard.html

[114] ELTEC Instruments, Inc., http://www.eltecinstruments.com/PDF/Ds/Data\%20Sheet\%20-

\%20Model\%20441.pdf 
[115] ELTEC Instruments, Inc., data sheet attached with the photodetector.

[116] S. Tavernier, "Experimental Techniques in Nuclear and Particle Physics" (Springer, Berlin, 2010) pp 23-53.

[117] http://spiff.rit.edu/classes/phys440/lectures/optd/optd.html

[118] http://www.helpsavetheclimate.com/climatetheory.html

[119] P. J. Kelly and R. D. Arnell, Vacuum 56, 159 (2000).

[120] M. Vopsaroiu, M. J. Thwaites, S. Rand, P. J. Grundy and K. O'Grady, IEEE Trans. Magn. 40, 2443 (2004).

[121] J. Sager, Optimisation of Heusler Alloy Thin Films for Spintronic Devices, PhD thesis, Department of Physics, University of York (2013).

[122] D. Tran, High target utilisation sputtering for the development of advanced materials for magnetic data storage applications, $\mathrm{PhD}$ thesis, Department of Physics, University of Exeter (2012).

[123] M. Vopsaroiu, G. V. Fernandez, M. J. Thwaites, J. Anguita, P. J. Grundy and K. O’Grady, J. Phys. D: Appl. Phys. 38, 490 (2005).

[124] M. Vopsaroiu, M. J. Thwaites, G. V. Fernandez, S. Lepadatu and K. O'Grady, J. Optoelectronics and Advanced Mater. 7, 2713 (2005).

[125] M. Henini (Editor), "Molecular Beam Epitaxy: From Research to Mass Production" (Elsevier, Oxford, 2013) p 2.

[126] J. R. Arthur, Surf. Sci. 500, 189 (2002).

[127] R. Kelsall, I. Hamley and M. Geoghegan (Eds.), "Nanoscale Science and Technology" (John Wiley and Sons, Ltd., Chichester, 2005) p 42.

[128] P. N. Prasad, "Nanophotonics" (John Wiley \& Sons, Inc., Hoboken, New Jersey, 2004), p 190.

[129] S. Kobayashi, The Rigaku J. 26, 1 (2010).

[130] K. Nagao and E. Kagami, The Rigaku J. 27, 2 (2011).

[131] A. Kirkland and J. L. Hutchison (Eds.), "Nanocharacterisation" (The Royal Society of Chemistry, Cambridge, 2007), p 1.

[132] A. Roy and J. Bhattacharya, "Nanotechnology in Industrial Wastewater Treatment" (IWA, London, 2015), p 48.

[133] D. B. Williams and C. B. Carter, "Transmission Electron Microscopy: A Textbook for Materials Science" (Springer, New York, 2009) $2^{\text {nd }}$ Ed., p. 154.

[134] J. Chureemart, Oriental and Thermal Stability of Advanced Recording Media, $\mathrm{PhD}$ thesis, Department of Physics, University of York (2013).

[135] E. E. Bragg and M. S. Seehra, J. Phys. E: Sci. Instrum. 9, 216 (1976).

[136] K. O'Grady, R. Chantrell and I. Sanders, IEEE Trans. Magn. 29, 286 (1993).

[137] J. Sagar, C. Yu, C. Pelter, J. Wood, L. Lari, A.Hirohata and K. O'Grady, IEEE Trans. Magn. 48, 11 (2012).

[138] A. Hirohata J. Sagar, L. Lari, L. R. Fleet and V. K. Lazarov, Appl. Phys. A 111, 423 (2013).

[139] A. Hirohata, H. Kurebayashi, S. Okamura, M. Kikuchi, T. Masaki, T. Nozaki, N. Tezuka and K. Inomata, J. Appl. Phys. 97, 103714 (2005).

[140] Y. Takamura, R. Nakane, H. Munekata and S. Sugahara, J. Appl. Phys. 103, 07D719 (2008).

[141] B. Balke, G. H. Fecher, H. C. Kandpal, C. Felser, K. Kobayashi, E. Ikenaga, J.-J. Kim and S. Ueda, Phys. Rev. B 74, 104405 (2006).

[142] Y. Fujita, S. Yamada, Y. Maeda, M. Miyao and K. Hamaya, Thin Solid Films 557, 386 (2014).

[143] L. R. Fleet, G. Cheglakov, K. Yoshida, V. K. Lazarov, T. Nakayama and A. Hirohata, J. Phys. D: Appl. Phys. 45, 032001(FTC) (2012). 
[144] J. Sagar, H. Sukegawa, L. Lari, V. K. Lazarov, S. Mitani, K. O'Grady and A. Hirohata, Appl. Phys. Lett. 101, 102410 (2012).

[145] Y. B. Xu and S. M. Thompson (Eds.), "Spintronic Materials and Technology" (CRC, New York, 2007) p 306.

[146] M Zander, J Herfort, K Kumakura, H-P Scöhnherr and A Trampert, J. Phys. D: Appl. Phys. 43, 305004 (2010).

[147] T. Onodera, M. Yoshida, N. Tezuka, M. Matsuura, S. Sugimoto and Y. Saito, Mat. Trans. 54, 1392 (2013).

[148] H. L. Yu and G. W. Yang, J. Appl. Phys. 109, 083509 (2011).

[149] N. Tezuka, N. Ikeda, F. Mitsuhashi and S. Sugimoto, Appl. Phys. Lett. 94, 162504 (2009).

[150] W. Wang, H. Sukegawa, R. Shan, T. Furubayashi and K. Inomata, Appl. Phys. Lett. 92, 221912 (2008).

[151] S. S. A. Hassan, Y. Xu, A. Hirohata, H. Sukegawa, W. Wang, K. Inomata and G. van der Laan, J. Appl. Phys. 107, 103919 (2010).

[152] T. F. Alhuwaymel, R. M. Abdullah, O. Whear, T. Huminiuc, R. Carpenter, M. ElGomati and A. Hirohata, IEEE Trans. Magn. 50, 2600504 (2014).

[153] Y. Sakuraba, T. Miyakoshi, M. Oogane, Y. Ando, A. Sakuma and T. Miyazaki, Appl. Phys. Lett. 89, 052508 (2006).

[154] J. Sagar, H. Sukegawa, L. Lari, V. K. Lazarov, S. Mitani, K. O'Grady and A. Hirohata, Appl. Phys. Lett. 101, 102410 (2012).

[155] C. T. Tanaka, J. Nowak and J. S. Moodera, J. Appl. Phys. 86, 6239 (1999).

[156] J. S. Correa, C. Eibl, G. Rangelov, J. Braun and M. Donath, Phys. Rev. B 73, 125316 (2006).

[157] R. B. Helmholdt, R. A. de Groot, F. M. Mueller, P. G. van Engen and K. H. J. Buschow, J. Magn. Magn. Mater. 43, 249 (1984).

[158] K. E. H. M. Hanssen, P. E. Mijnarends, L. P. L. M. Rabou and K. H. J. Buschow, Phys. Rev. B 42, 1533 (1990).

[159] M. P-Michalska, Acta Phys. Polonica A 103, 393 (2003).

[160] I. Maskery, Spin densities in 4 f and $3 d$ magnetic systems, $\mathrm{PhD}$ thesis, Department of Physics, University of Warwick (2013). 